\title{
Design Optimisation and Performance Evaluation of Flux Switching Machines for Geared Medium-Speed Wind Generator Drives
}

\author{
by \\ Udochukwu Bola Akuru \\ Dissertation presented in fulfilment of the requirements for the \\ degree of Doctor of Philosophy in Electrical Engineering \\ in the Faculty of Engineering at Stellenbosch University \\ Department of Electrical and Electronic Engineering \\ Stellenbosch University \\ Private Bag X1, Matieland 7602, South Africa
}

Supervisor: Prof. Maarten J. Kamper

December 2017 


\section{DECLARATION}

By submitting this thesis electronically, I declare that the entirety of the work contained therein is my own original work, and that I am the sole author thereof (save to the extent explicitly otherwise stated), that reproduction and publication thereof by Stellenbosch University will not infringe any third party rights and that I have not previously in its entirety or in part submitted it for obtaining any qualification.

\section{Udochukwu B. Akuru}

December 2017 


\title{
ABStract
}

\section{Design Optimisation and Performance Evaluation of Flux Switching Machines for Geared Me- dium-Speed Wind Generator Drives}

\author{
U. B. Akuru \\ E260, Department of Electrical and Electronic Engineering, Faculty of Engineering, Stellenbosch University, Stellen- \\ bosch 7602, South Africa \\ Dissertation: PhD (Electrical Engineering) \\ December 2017
}

As wind turbines become large, the cost of energy increases because of the employed drivetrain (geared or direct-drive). Consequently, non-conventional geared medium-speed (MS) generators are gaining relevance, potentially due to better compromise to both the generator and gearbox costs. The study proposes a novel approach for the multi-objective design optimisation (MDO) of two variants of geared MS flux switching machines (FSMs) in their simple radial-flux structures - the 12-stator slots/10-rotor poles (12/10) and 12/14 machines, with major emphasis on rare-earth-free designs for industrial-scale applications. Based on finite element analyses (FEA), whereby torque density, torque ripple and power factor are prioritised, multiple design options are provided in different Pareto maps for the designer to make informed selections. From an initial optimal design comparison of the 12/10 machines based on rare-earth permanent magnets (PMs) in different wind generator drivetrains at 10 $\mathrm{kW}$, the MS design yielded the best solution in terms of average torque densities per generator costs. Consequently, the focus on MS drivetrains was intensified to further evaluate the 12/10 and 12/14 rare-earth PM-FSMs as their power level shifts from $10 \mathrm{~kW}$ to $3 \mathrm{MW}$. As an indication of potentially adopting rare-earth-free designs, an improvement in terms of increased torque densities and reduced torque ripple values is obtained for the rare-earth designs at $3 \mathrm{MW}$ due to a number of factors such as variations in their PM volumes and relative differences in their saliency ratios. Moreover, due to the optimal behaviour of key design parameters in the MDO environment, the superior performance of the 12/14 machines at $10 \mathrm{~kW}$ are reversed at $3 \mathrm{MW}$. Disappointingly, based on the same design requirements, the nominated rare-earth-free designs such as ferrite PMs and wound-field (WF) coils do not produce better torque ripple effects compared to rare-earth PMs, as should be expected for such flux-focusing machines. However, an inherent tradeoff was found between their aspect ratios and armature current densities which influence the active mass, especially for industrialscale designs. Consequently, to ensure the feasibility of the optimal design, it may be needful to appropriately restrict the boundaries of the aspect and split ratios before engaging them in any MDO procedure. In another instance, it is found that it may be better to pursue MDO problems e.g., of WFFSMs, by concentrating more on the performance (torque ripple and power factor) than on the cost of the machines. Interestingly, it was also found that the cheapest MS generator, even when compared with similar conventional wind generators at $3 \mathrm{MW}$, is the WF-FSM. Eventually, to validate the series of FEA prediction made during the study, a $10 \mathrm{~kW}$ WF-FSM generator prototype is selected, manufactured and tested, with certain novel implementations. Based on measured no-load, shortcircuit, thermal, uncontrolled-normal and overload resistance, as well as current-controlled tests, the design feasibility as well as the conceptualisation of the proposed wind generator drivetrains has been proven beyond reasonable doubt. In agreement with Chen et al (2011) [44], there is, indeed, a bright future for FSMs. The study is concluded with a general conclusion and recommendations for the future. 


\title{
UITTREKSEL
}

\section{Ontwerp-Optimering en Vermoë Evaluering van Vloed Omskakelende Masjiene vir Geratte Medium-Spoed Wind Generator Dryfstelsels}

\author{
U. B. Akuru \\ E260, Departement Elektriese en Elektroniese Ingenieurswese, Universiteit van Stellenbosch, Stellenbosch 7602, Suid- \\ Afrika \\ Proefskrif: PhD (Elektriese Ingenieurswese) \\ Desember 2017
}

Soos hedendaagse wind turbines toeneem in grote, neem die koste van kragopwekking deur so ' $\mathrm{n}$ turbine ook toe weens die aandryfstelsel wat gebruik word (ratkas of direk-gedrewe sisteme). As gevolg hiervan word daar gekyk na nie-konvensionele, medium-spoed (MS) turbines wat toegerus is met ' $\mathrm{n}$ ratkas. Dit rede hiervoor is die goeie kompromi aangaande die generator en ratkas kostes. In hierdie studie word ' $n$ nuwe benadering tot multi-objektiewe ontwerp-optimering (MOO) vir twee verskillende geratte MS vloed-omskakelende masjiene (VOM), wat in hul mees eenvoudige radiale-vloedstrukture-die 12-stator gleuf/ 10 rotor pole (12/10) en 12/14 masjiene, met 'n groot klem op skaars-aarde-vrye ontwerpe vir industriële toepassings. Gebaseer op eindige element ontledings (EEO), waardeur wringkragdigtheid, wringkrag-rimpeling en arbeidsfaktor geprioritiseer word, word verskeie ontwerpopsies verskaf vir die ontwerper om ingeligte keuses te maak, in verskillende Pareto kaarte. Vanuit 'n voorlopige vergelykende studie van die 12/10 masjien, wat gebaseer is op ' $\mathrm{n}$ skaars-aarde permanente magnete (PM) en toepassing vind in verskillende $10 \mathrm{~kW}$ turbines, is gevind dat die MS ontwerp die beste oplossing gelewer het in van gemiddelde wringkragdigtheid per generatorkoste. Gevolglik is die fokus op MS-turbines versterk om die 12/10 en 12/14 skaars-aarde PM-VOMs te evalueer, soos hul kraguitset van $10 \mathrm{~kW}$ na $3 \mathrm{MW}$ vermeerder. ' $\mathrm{n}$ Aanduiding dat skaars-aarde-vrye ontwerpe gebruik kan word in pleks van die huidige standaard, is die verbeteringe in terme van verhoogde wringkragdigtheid en verminderde wringkrag rimpelwaardes, wat verkry is vir die skaars-aarde ontwerpe by $3 \mathrm{MW}$, weens ' $\mathrm{n}$ aantal faktore soos die variasies in hul PM volumes en die relatiewe verskille in hul speek verhoudings. Verder, weens die optimale gedrag sleutel-ontwerpsparameters in die MOO, word daar gevind dat die voortreflike vermoë van die $12 / 14$ masjien by $10 \mathrm{~kW}$ omgekeerd is by $3 \mathrm{MW}$. Dit was egter teleurstellend dat, as gevolg van die selfde ontwerpskriteria, die benoemde skaars-aarde-vrye ontwerpe soos die ferrietPMs en bewikkelde veld (BV) spoele nie beter wringkrag-rimpel-effekte in vergelyking met die skaars-aarde-vrye ontwerpe lewer nie; soos verwag word vir sulke vloed-gekonsentreerde masjiene. Nogtans, daar was ' $n$ inherente uitruiling gevind tussen die aspekverhoudings en ankerstroomdigtheid wat ' $n$ invloed het op die aktiewe massa, veral binne industriële-skaal ontwerpe. Gevolglik, om die haalbaarheid van die optimale ontwerp te verseker, kan dit nodig wees om die grense van die aspek en verdeelverhoudings behoorlik te beperk voordat hulle in enige MOO-prosedure betrek word. In 'n ander geval word gevind dat dit beter kan wees om MOO-probleme, bv. Van BVVOMs, te volg, deur meer op die prestasie (wringkrag rimpel en arbeidsfaktor) te konsentreer as op die koste van die masjiene. Interessant genoeg is gevind dat die goedkoopste MS-generator, selfs as dit vergelyk word met soortgelyke konvensionele wind generators teen $3 \mathrm{MW}$, die BV-VOM is. Uiteindelik, om die reeks EEO-berekeninge van die die studie te bevestig, word 'n $10 \mathrm{~kW} \mathrm{BV-}$ VOM-generator prototipe gekies, vervaardig en getoets, met sekere nuwe implementerings. Gebaseer op die gemete geen-las, kortsluit, termiese, onbeheerd-normaal- en oorlas-weerstand, sowel as die stroom-beheerde toetse, is die ontwerpsuitvoerbaarheid sowel as die konsepsie van die voorgestelde wind turbine dryfstelsels buite redelike twyfel bewys. In ooreenstemming met Chen et al (2011) [44] is daar inderdaad 'n blink toekoms vir VOMs. Die studie word afgesluit met met 'n algemene gevolgtrekking en aanbevelings vir die toekoms. 


\section{ACKNOWLEDGEMENTS}

In reaching this stage in my life where the final production of this dissertation has become possible, I will like to thank the following persons, groups or entities who have made the journey possible for me, perhaps, not in any specified order:

- My supervisor Prof. Kamper for his excellent supervision and foremost support towards this venture. The words to express my gratitude are reserved for another volume. Thank you!

- My former HOD, Engr. Dr. B. O. Anyaka, I honestly cannot forget your help.

- The former Vice-Chancellor, University of Nigeria, Prof. Bartho Okolo.

- My amiable wife, My Honey, Eunice, and just to save space, to our son, Zion IK, who later joined us during my $\mathrm{PhD}$ research. I always have you both in my heart.

- My parents, Mr. and Mrs. S. N. Akuru. I'm happy that the Lord God I serve has preserved you both to enjoy this achievement with me!

- My late uncle, Mr. Peter Akuru who departed midway into my PhD study. I can't forget your impact at critical points in my life. Sincerely, I'm lost for words at your untimely departure.

- My siblings, cousins, in-laws and other extended members of the Akuru family, too numerous to mention individually, you're much loved and appreciated!

- The large body of believers in my spiritual network who have made my life sweet, with a host of close friends who have stuck closer like brothers and sisters, The Deeper Life Family...

- Professors O. I. Okoro and A. O. E. Animalu, two-of-a-kind. I can never forget the research seeds you both sowed in me when I was nothing in the academic space!

- All my teachers growing up... e.g., Uncle Bayo, Aunty Shola, Mrs. Rose, Bro. Isaac, Mr. Ayoola, etc. You all believed in me when I was nothing, I owe so much to your trusts.

- My senior brother and mentor, Engr. Ben Okah, former Honourable Commissioner for Public Utilities, Ebonyi State, Nigeria, for your kindness and selflessness.

- The entire staff of Electrical Engineering Department, University of Nigeria, under the immediate-past headship of Engr. Prof. E. C. Ejiogu and the current headship of Engr. Prof. E. S. Obe, my M.Eng supervisor; I cannot individually evaluate everyone's unalloyed support.

- Kenan Cloete for his assistance in developing the prototype manufactured in this thesis, in conjunction with André Swart, Petro Petzer, Murray Jumat, Tsepang Jebetle and Christoph Botha, for their support at various stages of the production, assembly and testing process.

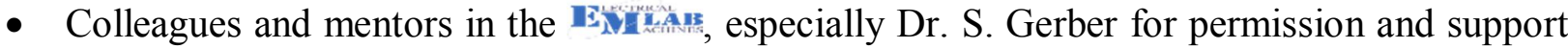
on the use of his FEA package (SEMFEM) in the course of my $\mathrm{PhD}$ research.

- Stellenbosch University, for the award of a Postgraduate Merit Bursary in 2015.

- The Government of Ebonyi State of Nigeria, for its foreign scholarship award.

- Confidants and pals over the years like Dr. (Mrs.) C. C. Esimone, Deji, Tony, Ifeanyi, Bamaiyi, Ola, Emma (both of them), Dr. Andrew at UKZN, etc.

- All my pastors and spiritual mentors superintended by Pastor W. F. Kumuyi, the general overseer of the Deeper Christian Life Ministry, worldwide.

- The University of Nigeria, my alma mater and the current Vice-Chancellor, Prof. Benjamin Chukwuma Ozumba.

- All my other professorial and family(ier) friends, and those who I have just missed their names here, for no justified reason, please, forgive me. You're all specially acknowledged!

The Lord bless you all in Jesus name, Amen. 


\section{Publications}

\section{Local conferences}

[1] U. B. Akuru and M. J. Kamper, "Investigation of Low-cost PM Flux Switching Machine for Medium-Speed Geared Wind Energy Applications," 2016 South African Universities Power Engineering Conference (SAUPEC) Proceedings, pp. 613-618, Stellenbosch, South Africa, 30 Jan.-1 Feb. 2017. Cited in text as reference [112].

\section{International conferences}

[2] U. B. Akuru and M. J. Kamper, "Performance Comparison of Optimum Wound-Field and Ferrite PM Flux Switching Machines for Wind Energy Applications," XXII ${ }^{\text {th }}$ International Conference on Electrical Machines (ICEM), Lausanne, Switzerland, pp. 2480-2487, 4-7 Sept. 2016. Cited in text as reference [111]. [Online]. Available: http://ieeexplore.ieee.org

[3] U. B. Akuru and M. J. Kamper, "Evaluation of flux switching PM machines for mediumspeed wind generator drives," 2015 IEEE Energy Conversion Congress and Exposition (ECCE), Montreal, Canada, pp.1925-1931, 20-24 Sept. 2015. Cited in text as reference [101]. [Online]. Available: http://ieeexplore.ieee.org

[4] U. B. Akuru and M. J. Kamper, "Comparative advantage of flux switching PM machines for medium-speed wind drives," 2015 International Conference on the Domestic Use of Energy (DUE), Cape Town, South Africa, pp.149-154, March 31 2015-April 1 2015. Cited in text as reference [98]. [Online]. Available: http://ieeexplore.ieee.org

[5] U. B. Akuru and M. J. Kamper, "Contemporary wind generators," 2014 International Conference on the Industrial and Commercial Use of Energy (ICUE), Cape Town, South Africa, pp.345-352, 19-20 Aug. 2014. Cited in text as reference [18]. [Online]. Available: http://ieeexplore.ieee.org

\section{Journal articles}

[6] U. B. Akuru and M. J. Kamper, "Formulation and Multi-Objective Design Optimisation of Wound-Field Flux Switching Machines for Wind Energy Drives," IEEE Transactions on Industrial Electronics, 2017 (in press). Cited in text as reference [125]. [Online]. Available: http://ieeexplore.ieee.org

[7] U. B. Akuru and M. J. Kamper, Contemporary wind generators. Journal of Energy in Southern Africa, vol. 26, no. 3, pp.116-124, Aug. 2015. Cited in text as reference [97]. [Online]. Available: http://www.scielo.org.za/pdf/jesa/v26n3/13.pdf 


\section{GRANTS}

- 2016 IEEE Region 8 Voluntary Contribution Fund Travel Grant to attend the XXII ${ }^{\text {th }}$ International Conference on Electrical Machines (ICEM 2016) held on 4-7 Sept. 2016 in Lausanne, Switzerland.

- 2016 IAS CMD Conference Student Travel and Publication Program Grant to attend XXII ${ }^{\text {th }}$ International Conference on Electrical Machines (ICEM 2016) held on 4-7 Sept. 2016 in Lausanne, Switzerland.

- 2015 IEEE ECCE Student Travel Grant to attend the IEEE Energy Conversion Congress and Exposition (ECCE) held on Sept. 20-24, 2015 in Montreal, Quebec, Canada.

- 2015 Stellenbosch University PGIO Overseas Conference Grant to attend the IEEE Energy Conversion Congress and Exposition (ECCE) held on Sept. 20-24, 2015 in Montreal, Quebec, Canada. 


\section{DEDICATION}

I dedicate this work to God the Almighty, the One who made me, and owns me-Galatians 1:15.

He caused the east wind to blow in the heaven; and by His power He brought in the south wind.

$* * * * * * *$ Psalm 78:26******* 


\section{CONTENTS}

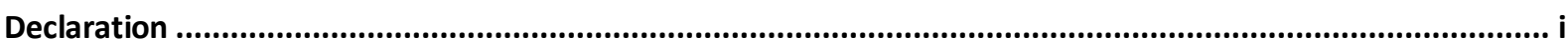

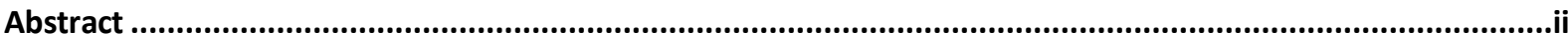

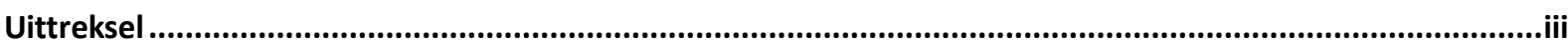

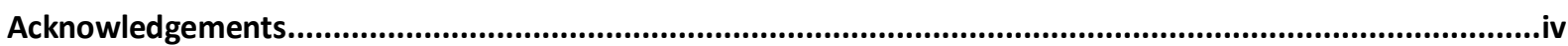

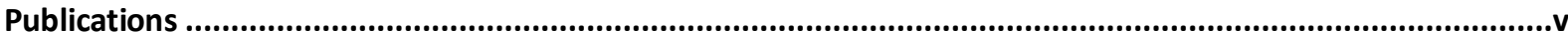

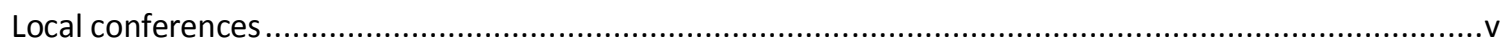

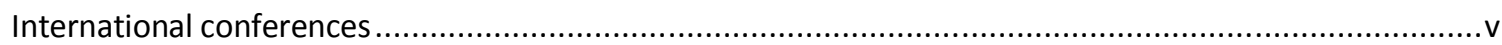

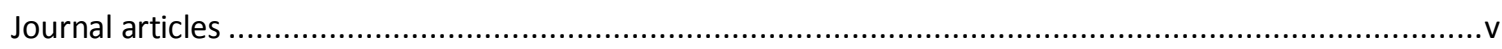

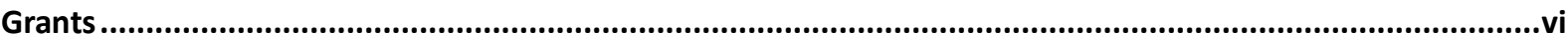

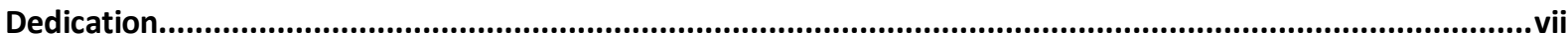

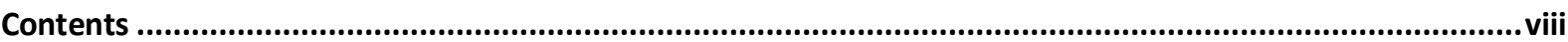

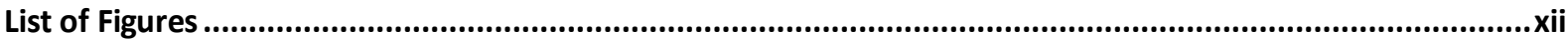

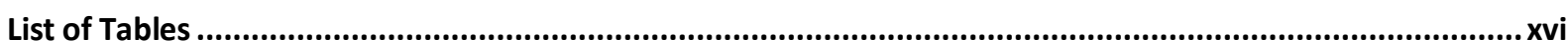

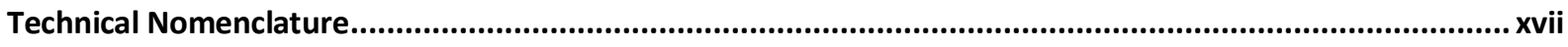

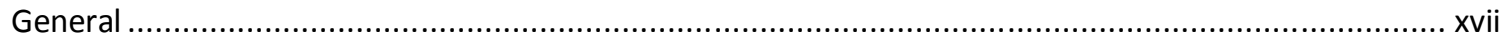

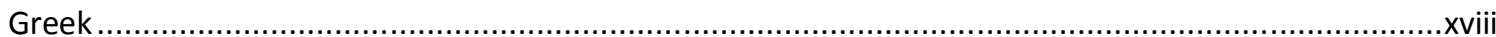

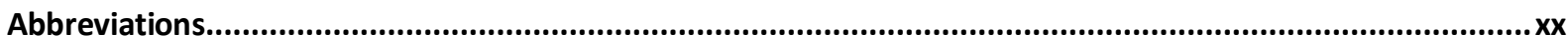

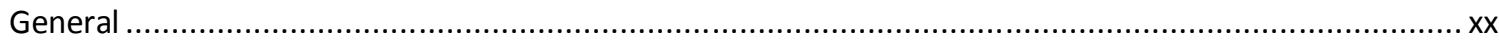

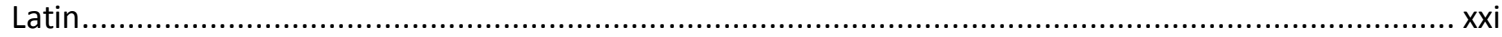

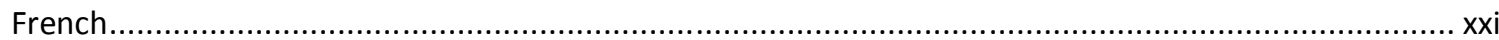

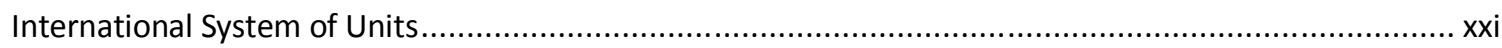

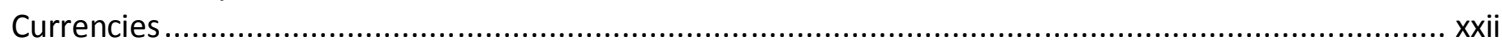

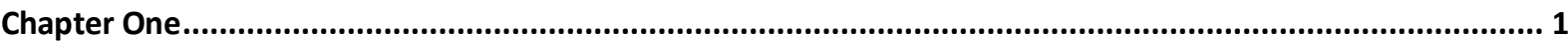

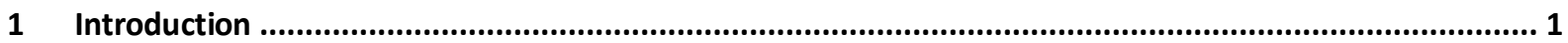

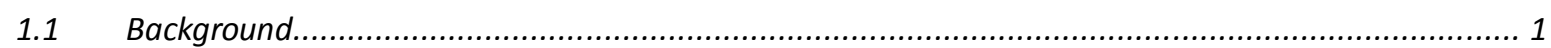

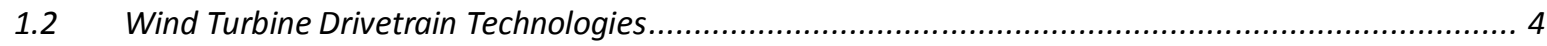

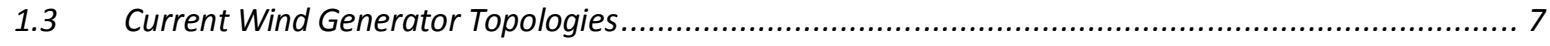

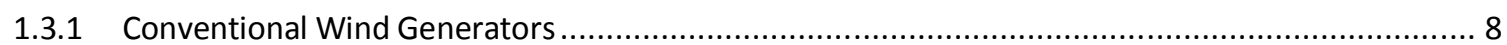

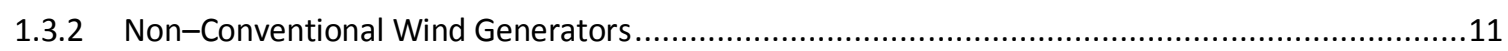

1.3.3 State-of-the-art on Flux Switching Machine Wind Generator Drives .........................................16 
1.4 Defining the Problem.

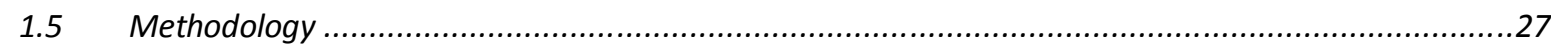

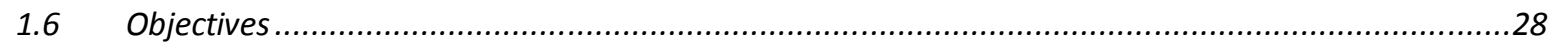

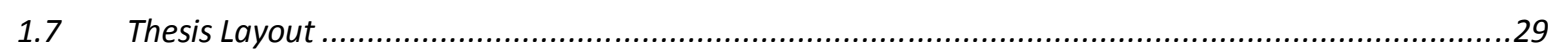

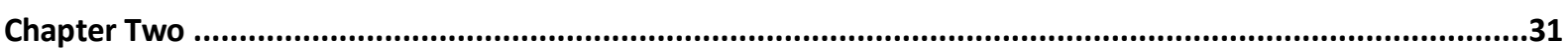

2 Drivetrain Performance and Comparison of PM-FSMs..................................................................31

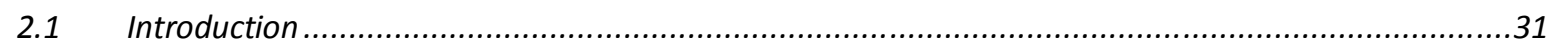

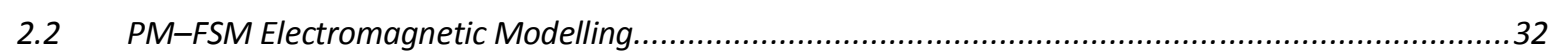

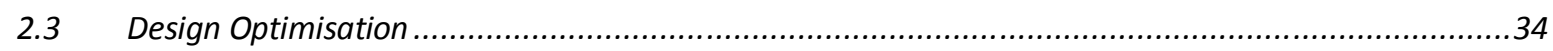

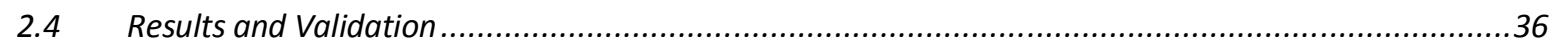

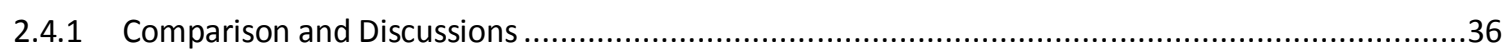

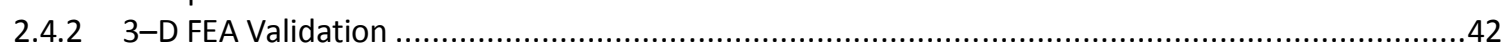

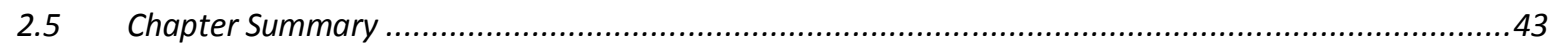

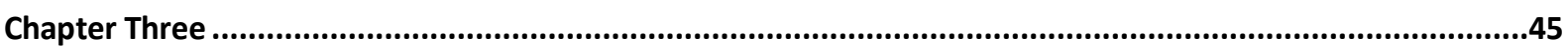

3 Design Optimisation and Evaluation of PM-FSMs .......................................................................45

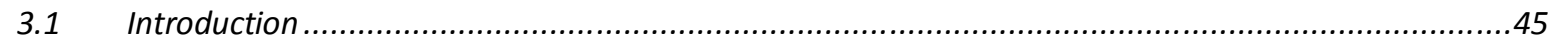

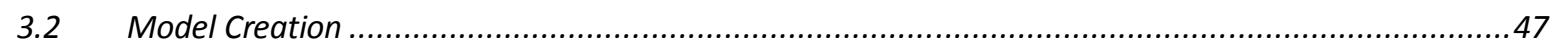

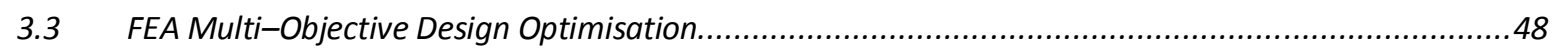

Implementation and Optimisation Results....................................................................49

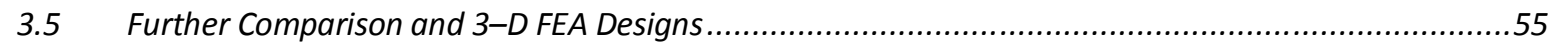

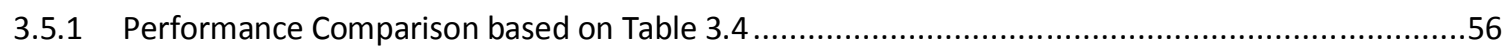

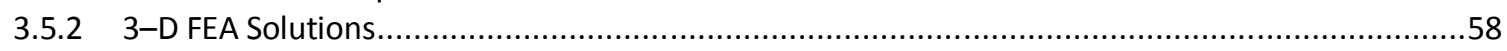

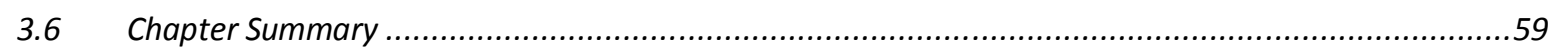

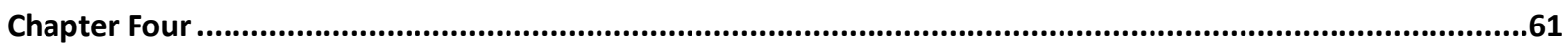

4 Performance Comparison and Investigation of Low-Cost Designs..................................................61

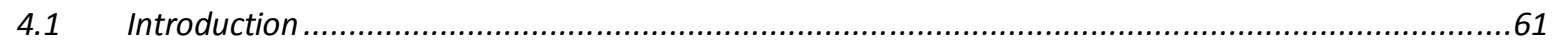

4.2 Electromagnetic Modelling and Design Optimisation ......................................................64

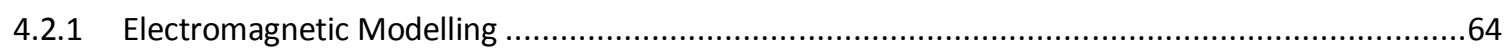

4.2.2 Design Optimisation Process, Results and Performance Comparison .......................................64

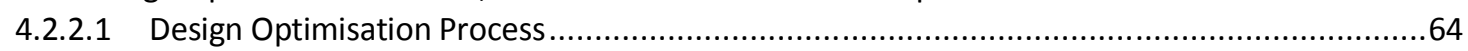

4.2.2.2 Optimisation Results and Performance Comparison .....................................................66 
4.3 Investigation of Ferrite PM-FSMs versus Rare-Earth PM-FSMs .......................................... 71

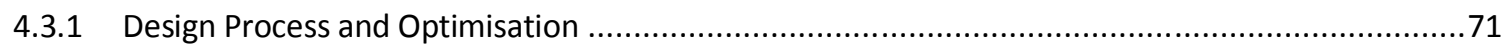

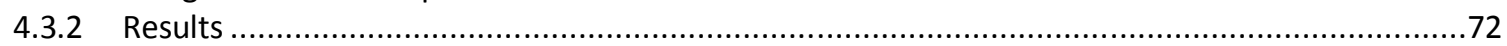

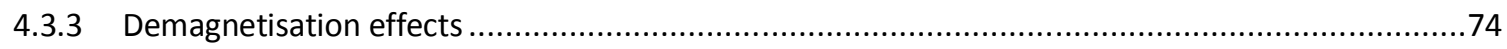

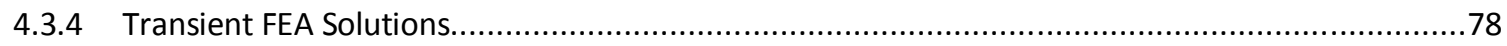

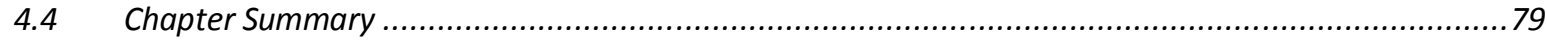

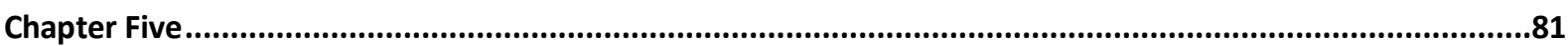

$5 \quad$ Formulation and Multi-Objective Design Optimisation of WF-FSM Wind Generators ...........................81

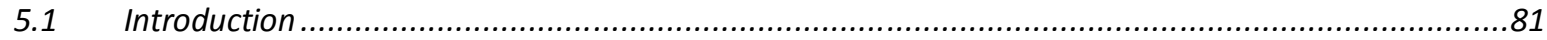

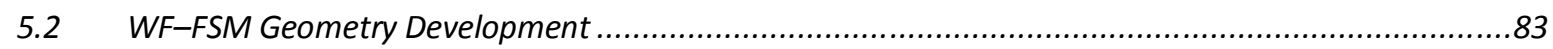

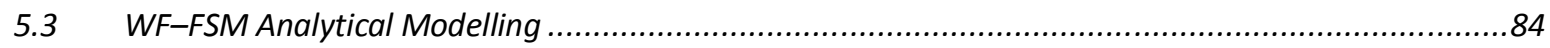

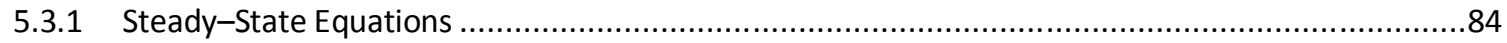

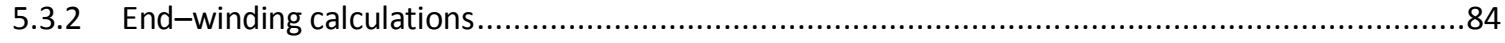

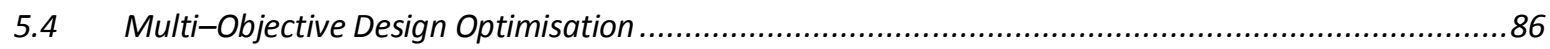

5.4.1 Optimisation Procedure and Problem Formulation ...........................................................86

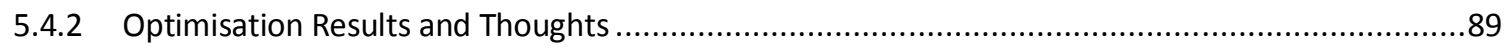

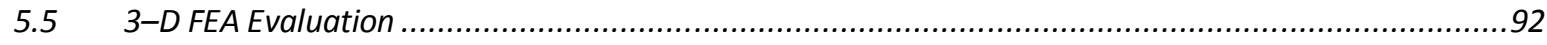

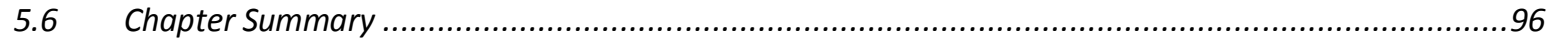

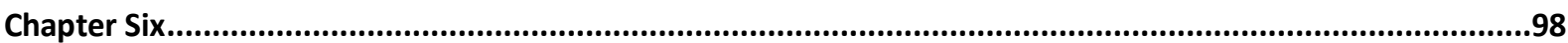

6 Design Characteristics and Potentials of Rare-Earth-Free FSMs for Large-Scale Wind Generator Drives .98

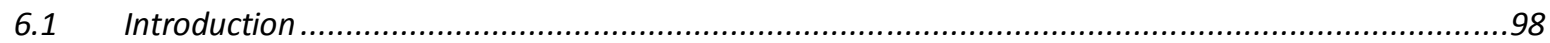

6.2 Design Optimisation Process..................................................................................... 100

6.3 Design Optimisation Results, Observations and Discussions ...............................................102

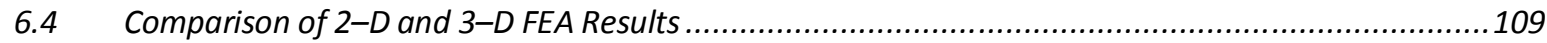

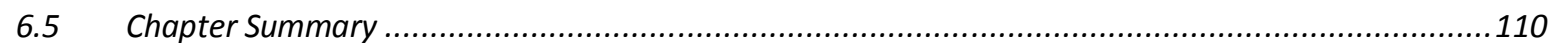

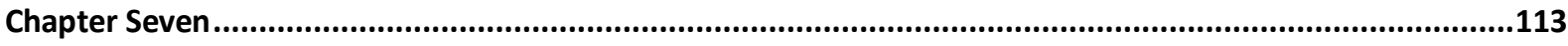

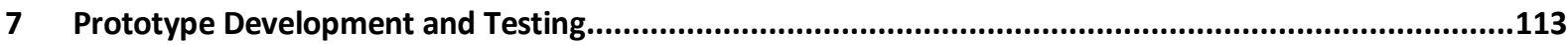

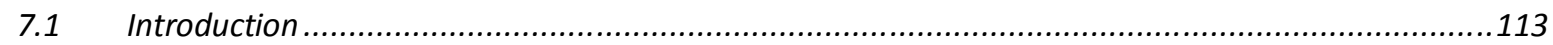

7.2 Prototype Presentation and Artistic Impression ....................................................... 114

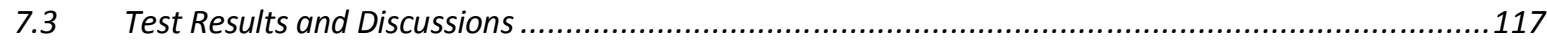

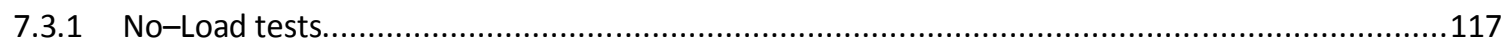

7.3.2 Short-Circuit Characteristics, Heat and Resistance Tests .....................................................124

7.3.3 Controlled Generator Mode and Voltage Regulation...........................................................129 


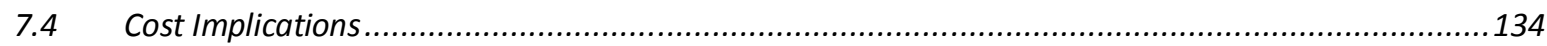

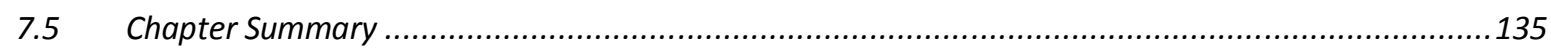

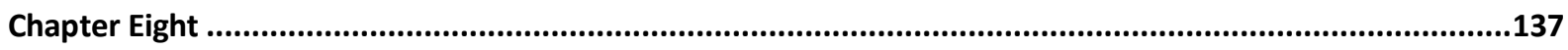

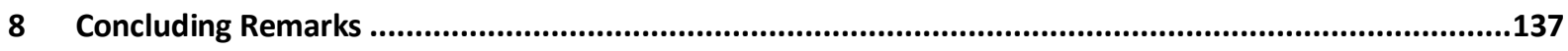

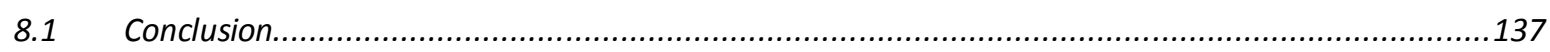

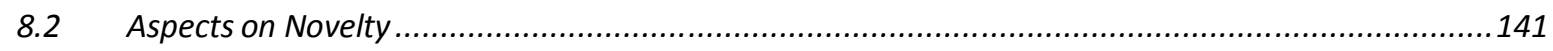

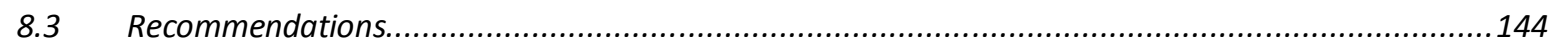

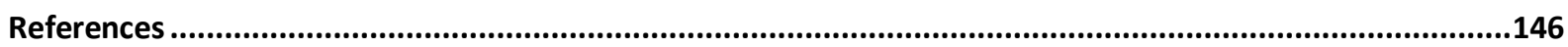

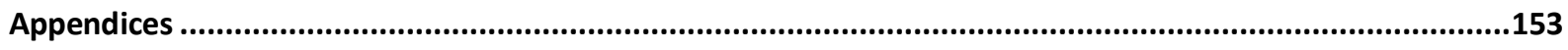

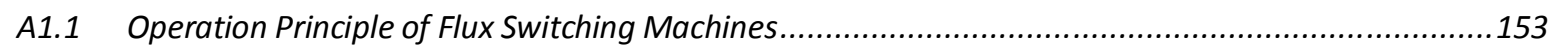

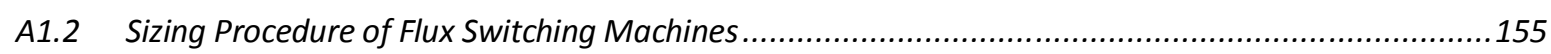




\section{LIST OF FIGURES}

Fig. 1.1. Global wind power capacity and annual additions, 2006-2016 [6]..................................................... 2

Fig. 1.2. Size and power increment in wind turbines since 1985 [8] ...........................................................

Fig. 1.3. Comparison in terms of mass and cost of energy of PMSG evaluated for different drivetrains at 4 MW [20]...... 6

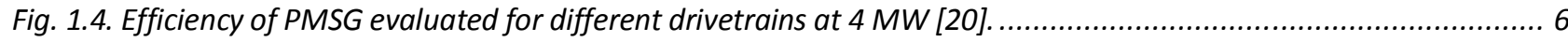

Fig. 1.5. Different wind generator drivetrains designed with induction generators [10]. ........................................ 9

Fig. 1.6. Wind generator drivetrains for different synchronous generator topologies [10]..........................................10

Fig. 1.7. Cross-sections of stator-active machines: (a) PM-DSM, (b) PM-FRM, and (c) PM-FSM................................

Fig. 1.8. Cross-section of the flux-switch alternator proposed in [40]. ..........................................................

Fig. 1.9. PM-FSM stator topologies: (a) E-core, (b) C-core, and (b) multi-tooth [44], [64]......................................19

Fig. 1.10. 12/10 PM-FSM: (a) all poles wound, and (b) alternate poles wound [65].................................................19

Fig. 1.11. Tree-diagram depicting the state-of-the-art in FSM wind generator drives...........................................22

Fig. 1.12. An illustration of the proposed geared medium-speed wind generator drivetrain. .....................................22

Fig. 1.13. Outline of the proposed 2-D FEA design optimisation process. .......................................................28

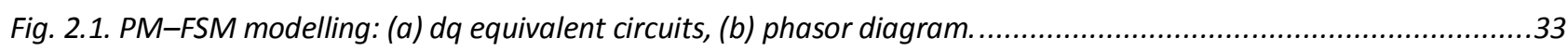

Fig. 2.2. Cut-out illustration of dq-axes rotor positions for 12/10 PM-FSM: (a) d-axis, and (b) q-axis..........................33

Fig. 2.3. Optimal design candidates presented for each PM-FSM drivetrain solution..................................................36

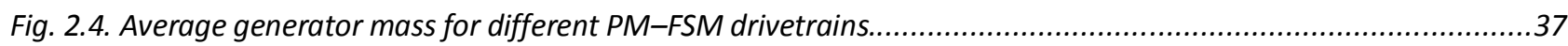

Fig. 2.5. Response of current density to power factor evaluated for different PM-FSM drivetrains............................39

Fig. 2.6. Efficiency versus power factor evaluated for the different optimal PM-FSM drivetrains. .................................39

Fig. 2.7. PM utilisation factor versus current density evaluated for different PM-FSM drivetrains...............................40

Fig. 2.8. Efficiency versus current density evaluated for different PM-FSM drivetrains..............................................41

Fig. 2.9. Efficiency versus optimal split ratios evaluated for different PM-FSM drivetrains.....................................41

Fig. 2.10. 3-D FEA model showing the magnetic flux lines at rated condition. ...................................................42

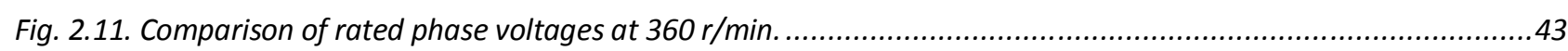

Fig. 3.1. All poles wound PM-FSM: (a) 12/10 design, and (b) $12 / 14$ design. .......................................................47

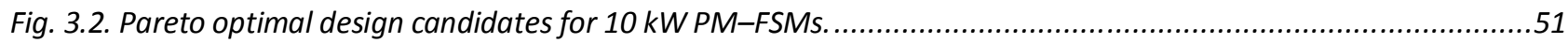

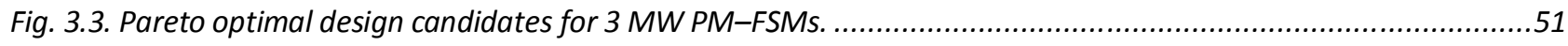

Fig. 3.4. Performance comparison of optimum design candidates of 12/10 $3 \mathrm{MW}$ machines at nomalised values and based on similar output power.

Fig. 3.5. Performance comparison of optimum design candidates of 12/14 $3 \mathrm{MW}$ machines at nomalised values and based on the same output power.

Fig. 3.6. Performance comparison of optimum design candidates of 12/14 3 MW machines at nomalised values, based on the torque ripple of design $I$.

Fig. 3.7. Comparison of PM amount used in $3 \mathrm{MW}$ optimum design candidates of $12 / 10$ and $12 / 14$ machines (1 per unit volume is the value of PM volume used in design I). .54

Fig. 3.8. Split ratios in selected 3 MW optimum design candidates. 54 
Fig. 3.9. Variation of current densities in selected $3 \mathrm{MW}$ optimum design candidates.

Fig. 3.10. Plots showing PM-FSMs load current profiles at optimum current angles against: (a) efficiency, and (b) power factor (1 per unit current is in terms of rated current in respective machine).

Fig. 3.11. Flux density maps of 3 MW PM-FSMs at rated conditions, analysed in 3-D transient FEA showing: (a) the 12/10 machine frozen at $5.21 \mathrm{~ms}$, and (b) the 12/14 machine frozen at $5.44 \mathrm{~ms}$.

Fig. 3.12. Load current versus torque characteristics of 3 MW PM-FSMs displayed for: (a) 12/10 machine, and (b) 12/14 machine (1 per unit current is in terms of rated current in respective machine).

Fig. 4.1. Global market for permanent magnets from 2013-2024 (volume in kilotons).

Fig. 4.2. Pareto optimal fronts for ferrite $P M-F S M$ showing plots of: (a) $M_{A}$ against $M_{P M}$ (b) $M_{P M}$ against $k_{\delta}$, and (c) $M_{A}$ against $k_{\delta}$

Fig. 4.3. Pareto optimal fronts for the WF-FSM showing plots of: (a) $M_{A}$ against $M_{F}$ (b) $M_{F}$ against $k_{\delta}$, and (c) $M_{A}$ against $k_{\delta}$

Fig. 4.4. Contrast between optimal aspect and split ratios. 68

Fig. 4.5. Comparison of different component costs. 70

Fig. 4.6. Cut-out cross-sectional views of magnetic field distributions at rated conditions analysed in the benchmarked optimal designs, viz., (a) ferrite PM-FSM, and (b) WF-FSM.......

Fig. 4.7. Plots of airgap flux densities under rated conditions.......................................................................... 70

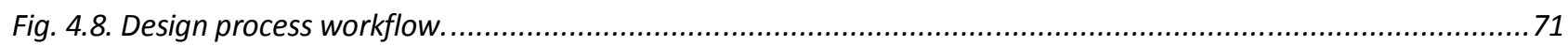

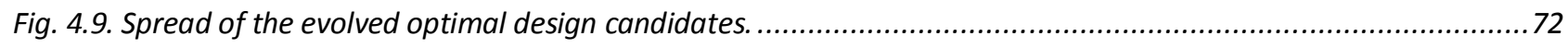

Fig. 4.10. Comparison of aspect ratio and split ratio in optimal PM-FSM variants. ............................................... 74

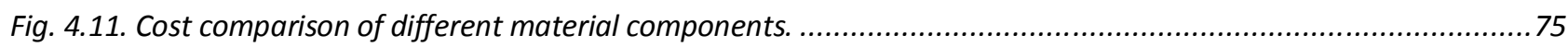

Fig. 4.12. 2-D static FEA display of PM reference point normal to flux lines along the $x$-direction for: (a) rare-earth PM-

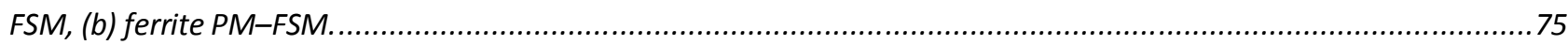

Fig. 4.13 Average PM flux density under different load conditions. .............................................................. 76

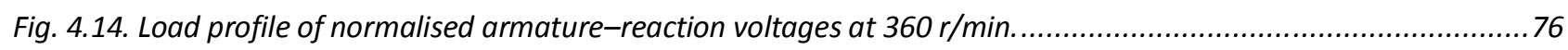

Fig. 4.15. Contour plots of PM flux densities at rated conditions: (a) rare-earth, and (b) ferrite. .................................77

Fig. 4.16. Comparison of flux linkages in rare-earth PM-FSM under no-load conditions. ........................................ 78

Fig. 4.17. Comparison of flux linkages in ferrite PM-FSM under no-load conditions.............................................. 78

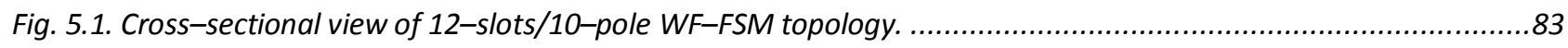

Fig. 5.2. Different WF-FSM end-winding projection: (a) airgap side, (b) radial cross-section, (c) outer perimeter surface and $(d)$ axial cut-out (Parts: $\boldsymbol{A}$ = field coil, $\boldsymbol{B}=$ phase coil, $\boldsymbol{C}=$ stator laminations). .................................................85

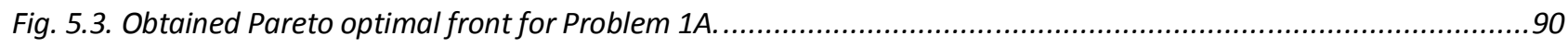

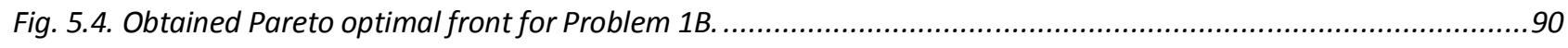

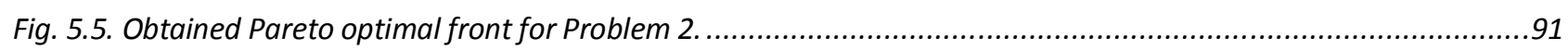

Fig. 5.6. Obtained Pareto optimal front for Problem 2, showing the torque ripple evaluated from purely 2-D FEA torque output. 93

Fig. 5.7. Per unit values of four optimum WF-FSMs selected from Problem 2 along the Pareto front. .94

Fig. 5.8. FEA display of: (a) finer 2-D mesh structures, (b) coarse 3-D mesh structures, (c) magnetic fields in 2-D static solution, and (d) flux density surface map in 3-D transient solution. 
Fig. 5.9. Nominal induced phase voltage waveforms at $360 \mathrm{r} / \mathrm{min}$ and the resulting frequency spectral harmonics in 2-D static and 3-D transient FEA

Fig. 6.1. Typical workflow processed in 2-D FEA (SEMFEM) simulation. 101

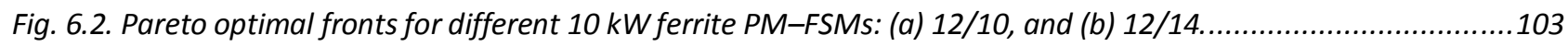

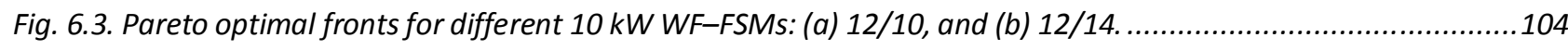

Fig. 6.4. Pareto optimal fronts for different 3 MW ferrite PM-FSMs: (a) 12/10, and (b) $12 / 14$...............................104

Fig. 6.5. Pareto optimal fronts for different 3 MW WF-FSMs: (a) 12/10, and (b) 12/14. 105

Fig. 6.6. Optimal partnership between current density and aspect ratio in $3 \mathrm{MW}$ rare-earth-free FSMs: (a) ferrite PMFSM, and (b) WF-FSM.. 106

Fig. 6.7. Magnetic flux distributions and densities of the compared 3 MW WF-FSMs displayed in: (a) 12/10 2-D model, (b) 12/10 3-D model, (c) 12/14 2-D model, and (d) 12/14 3-D model. 109

Fig. 6.8. On-load phase flux linkage waveforms plotted over half electrical period in 3 MW WF-FSMs: (a) 12/10 design, and (b) 12/14 design...... 110

Fig. 7.1. A block diagram of the drivetrain test-bench for the $10 \mathrm{~kW}$ WF-FSM prototype. 114

Fig. 7.2. An artistic impression of the proposed test-rig with highlights on: (a) the $10 \mathrm{~kW} W F-F S M$, (b) the torque sensor, (c) the coupling device, and (d) the $22 \mathrm{~kW}$ IM prime-mover.

Fig. 7.3. 3-D exploded views showing the different components in: (a) the stator assembly, and (b) the rotor assembly.

Fig. 7.4. Manufacturing and assembly: (a) laser cut puzzle-like modular stator lamination units, (b) laser cut simple rotor lamination, and (c) process of stator stack assembling......

Fig. 7.5. Manufacturing and assembly: (a) machined rotor hub and rotor guide pin affixed to laser cut end-plate, (b) assembling of wound-fields and stacked stator core, and (c) vanished stator core assembled with wound-fields and phase coils.

Fig. 7.6. Finalised assembly: (a) rotor, end plates, bearings and hubs, (b) stator, end plates, coils, back plate and support base, and (c) complete stator and rotor assembly. 120

Fig. 7.7. Parallel circuit devised for the DC wound-field coils in the constructed prototype. 121

Fig. 7.8. Implemented phase winding layout in the constructed prototype. 121

Fig. 7.9. Experimental test-bench used for actualising measurements on the constructed prototype........................122

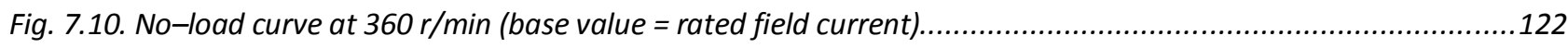

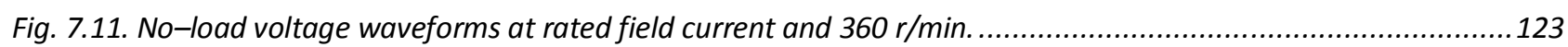

Fig. 7.12. Implementation of the Wye-connected three-phase short-circuits. 124

Fig. 7.13. Short-circuit characteristics of the $10 \mathrm{~kW}$ WF-FSM prototype operated at $360 \mathrm{r} / \mathrm{min}$ (base value = rated field current). 125

Fig. 7.14. Short-circuit current waveforms of the $10 \mathrm{~kW}$ WF-FSM prototype operated at rated field current and 360 $r / \min$.

Fig. 7.15. Representation of the different temperature hotspots: (a) Top projection on DC coil along the axial path, (b) end-winding knee on DC coil, and (c) top projection on AC coil on the radial side.

Fig. 7.16. Contour maps of instantaneous temperature readings observed after 90 minutes in the different hotspots: (a) Spot 1, (b) Spot 2, and (c) Spot 3. 
Fig. 7.17. Patterns of temperature rise during short-circuit operation in the major hotspots of the $10 \mathrm{~kW}$ WF-FSM prototype when operated at rated field current and $360 \mathrm{r} / \mathrm{min}$..

Fig. 7.18. Snapshot of very low-profile thermal activity in the surrounding stator lamination stack.

Fig. 7.19. Load resistance connection in uncontrolled load tests.

Fig. 7.20. Obtained current and voltage waveforms of the $10 \mathrm{~kW}$ WF-FSM prototype under load of 0.8 Ohms per phase operated without field-oriented control at rated field current and $360 \mathrm{r} / \mathrm{min}$.

Fig. 7.21. Current and voltage waveforms of the $10 \mathrm{~kW}$ WF-FSM prototype under uncontrolled-overload (10 Ohms per phase) operating condition at rated field current and $360 \mathrm{r} / \mathrm{min}$.

Fig. 7.22. Sample data measured on oscilloscope showing rotor alignment at $\sim 180 \mathrm{r} / \mathrm{min}$ and rated field and phase currents. 130

Fig. 7.23. Comparison of FEA and experimental results of the load current characteristics on the phase voltages for the WF-FSM prototype at $180 \mathrm{r} / \mathrm{min}$.

Fig. 7.24. Comparison of FEA and experimental results of the load current characteristics on the average torque evaluated for the WF-FSM prototype.

Fig. 7.25. Comparison of FEA and experimental results of the load current characteristics on torque ripple for the WFFSM prototype.

Fig. 7.26. Comparison of FEA and experimental results of the load current characteristics on efficiency for the WF-FSM prototype at $\sim 180 \mathrm{r} / \mathrm{min}$.

Fig. 7.27. Comparison of FEA and experimental results of the load current characteristics on power factor for the WFFSM prototype at $180 \mathrm{r} / \mathrm{min}$.

Fig. 7.28. Mass and cost of materials used in the manufacturing of the $10 \mathrm{kW-FSM}$ prototype.

Fig. 7.29. Distribution of costs among the different parts of the manufactured prototype (base value = total material cost of manufactured prototype).

Fig. A1.1. An illustration of the flux-switch concept in 2-D FEA (with field distributions) considered at different rotor positions such as: (a) $A$, and (b) $B$.

Fig. A1.2. Flux density map of 4-stator slots/6-rotor poles single-phase FSM at different rotor positions such as: (a) position " $A$ ", and (b) position " $B$ ".

Fig. A1.3. Sinusoidal expression of flux linkage, induced voltage, and load current plotted against the rotor position of single-phase flux-switch alternator, at $400 \mathrm{r} / \mathrm{min}$.

Fig. A1.4. Conceived geometry of the stator segments for: (a) PM-FSM design, and (b) WF-FSM design. 156

Fig. A1.5. Conceived geometry of the simple rotor lamination. 157 


\section{LIST OF TABLES}

Table 1.1. Comparison of the different drivetrain concepts.

Table 1.2. Quantitative comparison of three major wind generators [4] .......................................................... 11

Table 1.3. Comparison of different novel stator-PM machines [44] ............................................................... 14

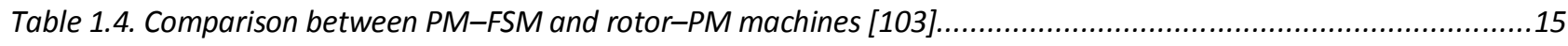

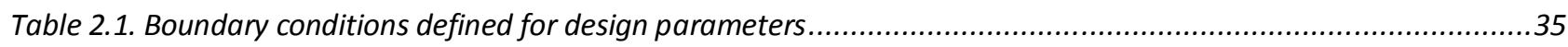

Table 2.2. Comparison of PM-FSM performance parameters under different drivetrains at $10 \mathrm{~kW} . . . . . . . . . . . . . . . . . . . . . . . . . .38$

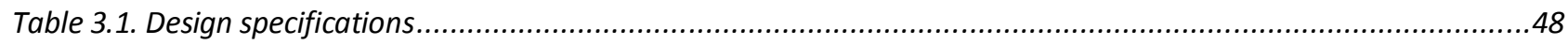

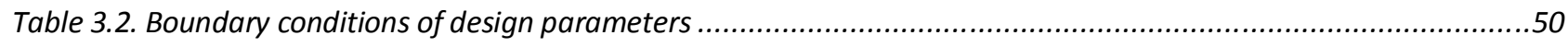

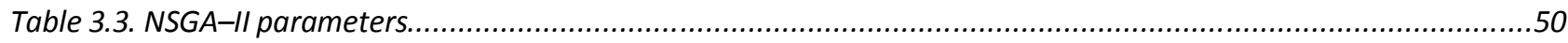

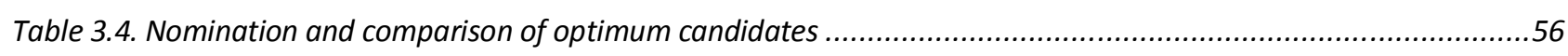

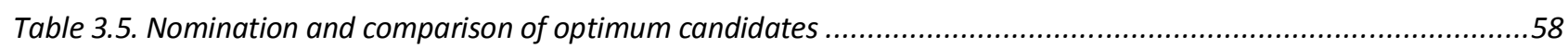

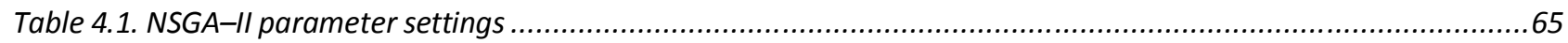

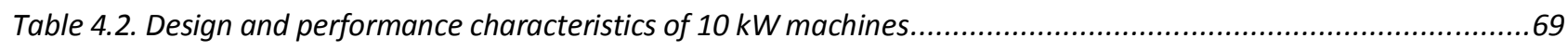

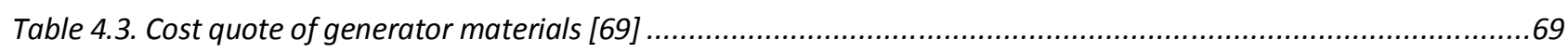

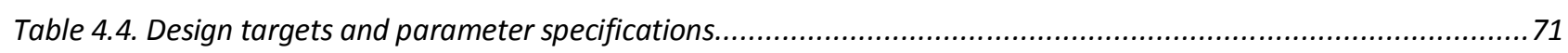

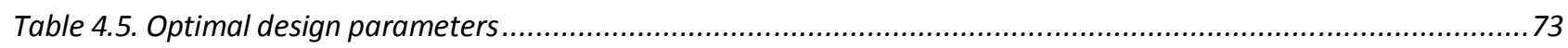

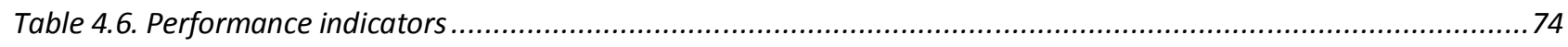

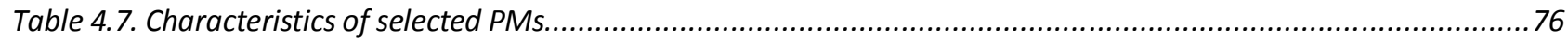

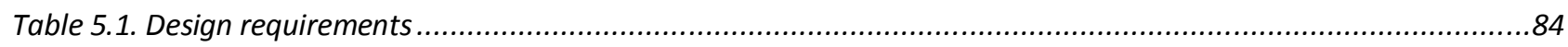

Table 5.2. Analytic calculations vs. FEA results for sampled $10 \mathrm{~kW}$ WF-FSM .......................................................85

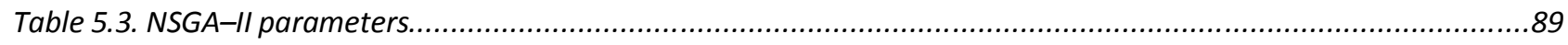

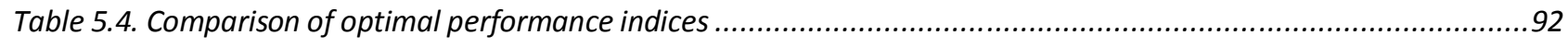

Table 5.5. Validation of optimal performance indices based on Design III.......................................................96

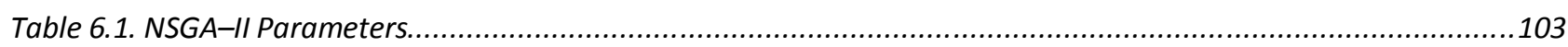

Table 6.2. Performance comparison of different machine characteristics for geared medium-speed wind generators.. 107

Table 6.3. Comparison of some basic optimal design parameters .................................................................107

Table 6.4. Comparison of performance characteristics of 3 MW WF-FSMs in 2-D and 3-D FEA...............................110

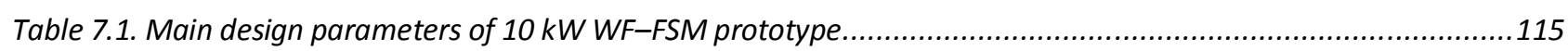

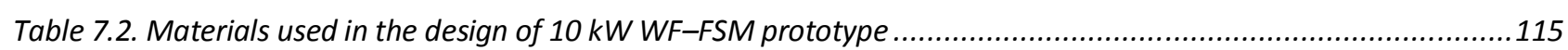

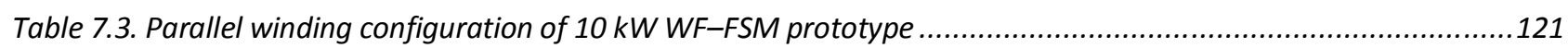

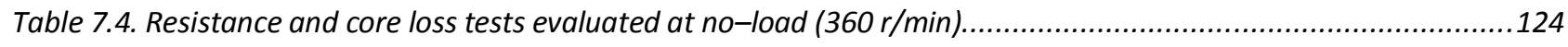

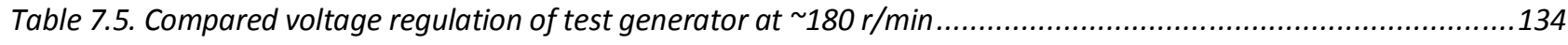




\section{TeChNical Nomenclature}

\section{General}

$A, B, C$

A, B

$A_{W}$

$a, b$, and $c$

$A_{F}$

$A_{p h}$

$b_{F}$

$\dot{B}_{g}$

$\dot{B}_{k}$

$b_{p m}$

$b_{p r}$

$B_{r}$

$b_{s l s}$

$c_{s}$

$C_{p}$

$C_{m}, \sigma$ and $\beta$

$D_{\text {in }}$

$D_{\text {out }}$

$D_{\text {rot }}$

$D_{s h}$

$e_{s}$

$e_{0}$

F

$f_{e}$

$g$

$G, G_{1}, G_{2}$

$H_{c}$

$h_{c}$

$h_{F}$

$h_{y r}$

$h_{y s}$

$I_{d}$ and $I_{q}$

$I_{F}$

$I_{S}$

$i_{s}$

$\mathrm{J}$

$\mathrm{J}_{F}$

K

$K_{M}$

$L_{d}$ and $L_{q}$

$L_{e}, L_{e(1)}$ and $L_{e(2)}$

$l_{e}, l_{e 1}, l_{e 2}$

$l_{e F}$

$l_{9}$

$l_{9 F}$ magnetic axis of the first, second and third phase of the stator winding threephase variables

random designations used in Tables 6.2 and 6.3 in Chapter 6

wind turbine rotor swept area

variables used to determine $K$

area of field wire

area of the phase wire

field core iron length in WF-FSMs

peak airgap flux density

peak flux density measured inside a corresponding $k$ iron core part

PM length in PM-FSMs

rotor pole width

PM remanence

slot opening width

stator tooth arc factor

wind turbine aerodynamic efficiency

Steinmetz coefficients for core loss estimation

stator inner diameter

stator outer diameter

rotor external diameter

shaft diameter

terminal voltage

internally generated voltage

vector of objective functions

fundamental frequency

airgap length

vector of inequality constraints

PM coercive force

height of the phase coil

field core iron width

rotor yoke height

stator yoke height

$\mathrm{d}-$ and $\mathrm{q}-$-axes phase currents

field current

RMS phase current

load current

phase current density

field current density

a constant used for end-winding calculations

a factor to account for cross magnetisation effects

$\mathrm{d}-$ and $\mathrm{q}-$ axes inductances

end-wing inductances

end-winding length on one side of the phase coils

end-winding length on one side of the field coil

full distance of the phase end-winding from the lamination stack

full the distance of the field end-winding coil from the lamination stack 


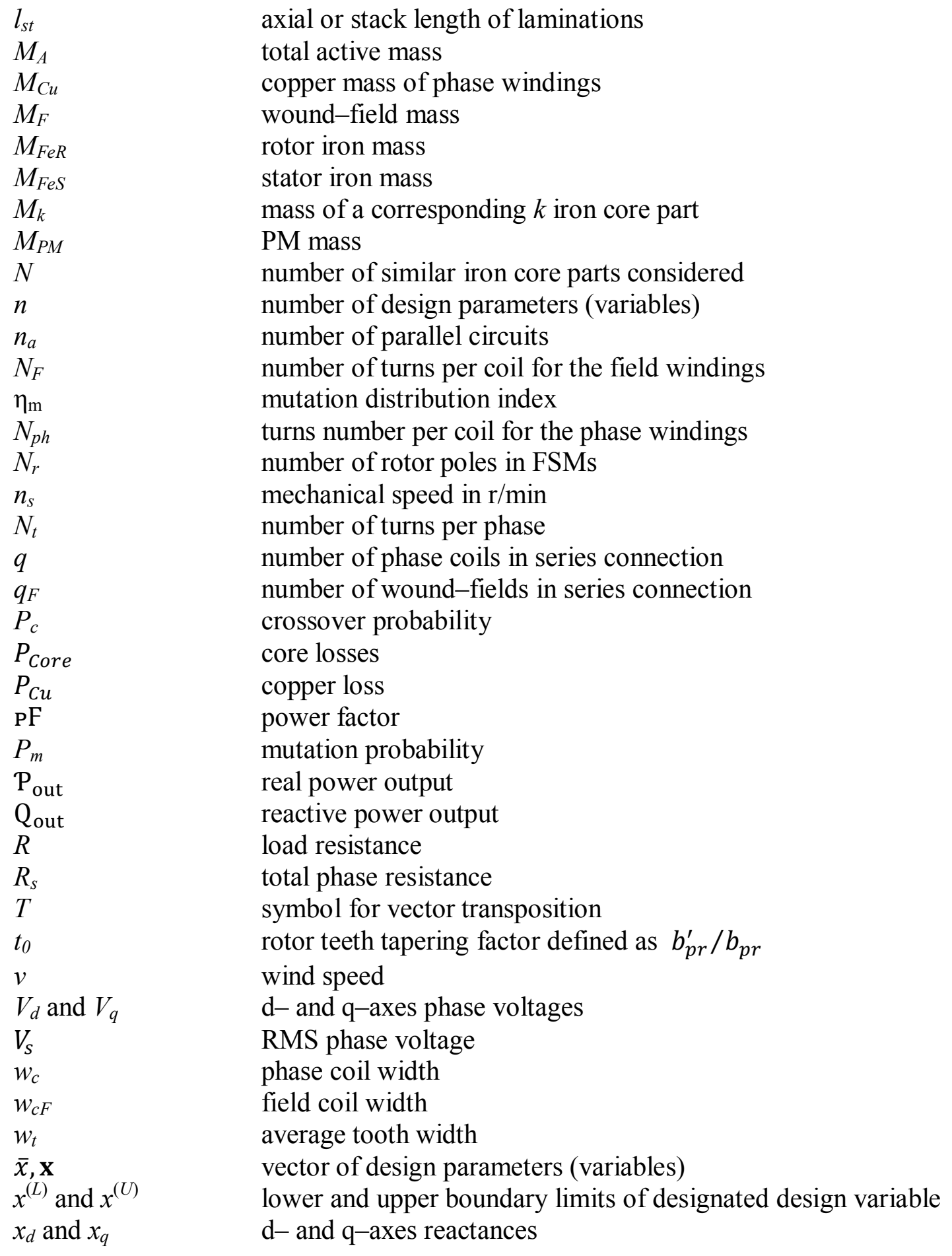

\section{Greek}

$\alpha$
$\Delta$
$\eta$
$\eta_{\mathrm{c}}$
$\theta$

$\theta_{p}$
$\theta_{\mathrm{sF}}$
$\theta_{\mathrm{sp}}$

current angle

load angle

efficiency

crossover distribution index

transformation angle representing the magnetic field axis (d-axis) of the rotor with respect to the magnetic phase $A$ vector or simply an angle used to determine the position of the rotor wind turbine blade pitch angle slot fill factor for field windings slot fill factor for phase windings 


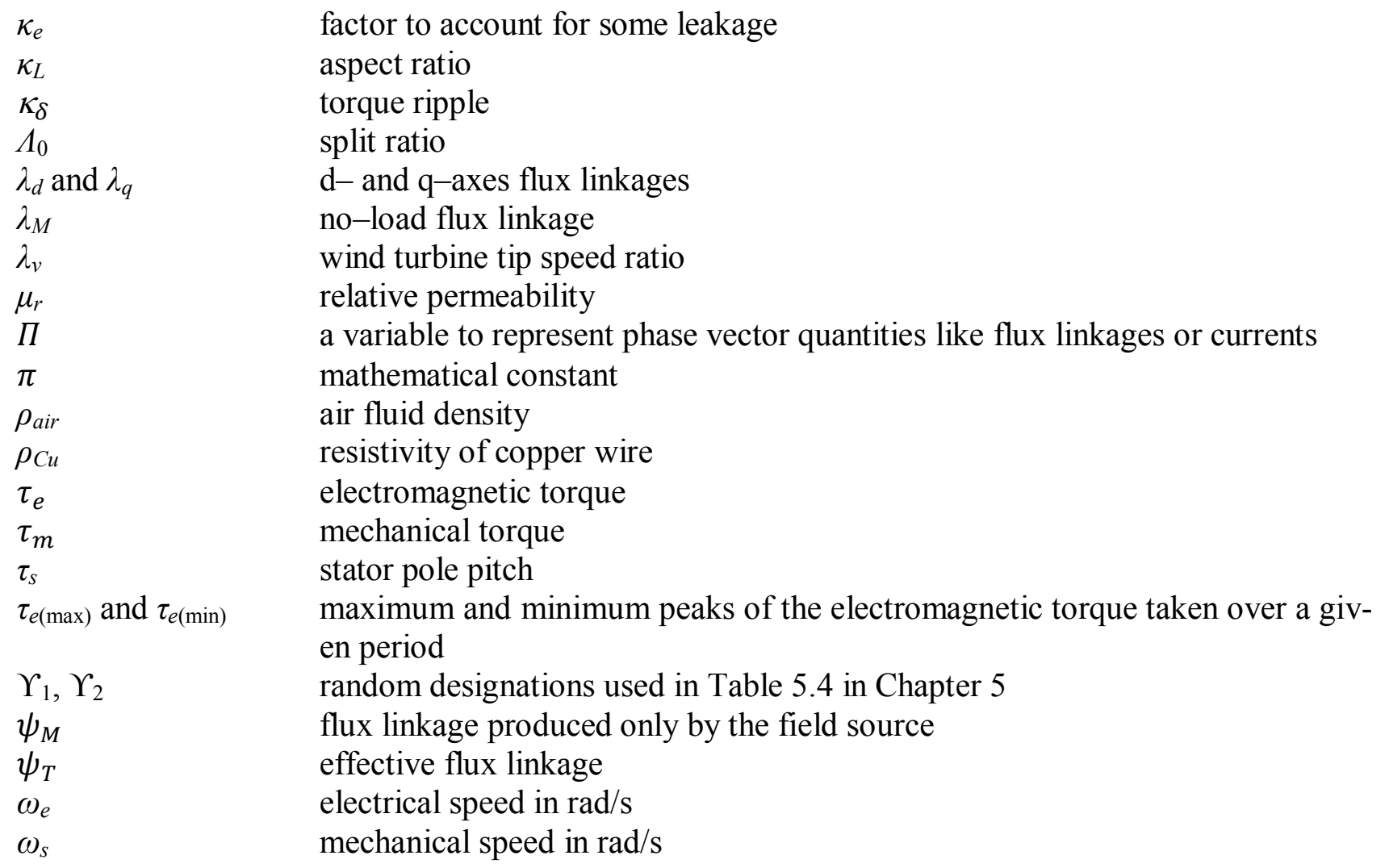




\section{Abbreviations}

\section{General}

a.k.a.

AFM

$\mathrm{AC}$

CAPEX

$\mathrm{CoE}$

CPU

EV

$\mathrm{d}-$ and $\mathrm{q}-(\mathrm{dq})$

DC

DD

DFIG

EESG

EMF

FEA

FP

FPC

G

GB

HAWT

HE-FSM

HEV

HS

HTS

IG

IM

IPM

LCOE

LS

MDO

MGPM

MMF

MS

$\mathrm{NdFeB}$

NSGA-II

OPEC

OPEX

PHEV

PM

PM-DSM

PM-FRM

PM-FSM

PMSG

PMSM

$\mathrm{pu}$

PWM

RAM
Also known as

Axial Flux Machine

Alternating Current

Capital Expenditure

Cost of Energy

Central Processing Unit

Electric Vehicle

Direct and Quadrature

Direct Current

Direct-Drive

Doubly-Fed Induction Generator

Electrical-Excited Synchronous Generator

Electromotive Force

Finite Element Analysis

Frozen Permeability

Fully-Rated Power Converter

Gearbox

Gigabyte

Horizontal Axis Wind Turbine

Hybrid-Excited Flux Switching Machine

Hybrid Electric Vehicle

High-Speed

High-Temperature Superconductor

Induction Generator

Induction Motor

Interior Permanent Magnet

Levelised Cost of Electricity

Low-Speed

Multi-objective Design Optimisation

Magnetically geared permanent magnet

Magnetomotive Force

Medium-Speed

Neodymium Iron Boron

Non-dominated Sorting Genetic Algorithm II

The Organisation of the Petroleum Exporting Countries

Operating expenditure

Plug-in Hybrid Electric Vehicle

Permanent Magnet

Permanent Magnet Double Salient Machine

Permanent Magnet Flux Reversal Machine

Permanent Magnet Flux Switching Machine

Permanent Magnet Synchronous Generators

Permanent Magnet Synchronous Motors

Per Unit

Pulse Width Modulation

Random Access Memory 


$\begin{array}{ll}\text { RFM } & \text { Radial Flux Machines } \\ \text { RMS } & \text { Root mean square } \\ \text { ROI } & \text { Return on Investment } \\ \text { SEMFEM } & \text { Stellenbosch Electrical Machines Finite Element Method } \\ \text { SCIG } & \text { Squirrel Cage Induction Generator } \\ \text { SG } & \text { Synchronous Generator } \\ \text { SmCo } & \text { Samarium Cobalt } \\ \text { SRG } & \text { Switched Reluctance Generator } \\ \text { SRM } & \text { Switched Reluctance Machine } \\ \text { SSC } & \text { Solid State Converter } \\ \text { SS-PMG } & \text { Slip Synchronous Permanent Magnet Generator } \\ \text { TFM } & \text { Transverse Flux Machine } \\ \text { THD } & \text { Total Harmonic Distortion } \\ \text { WF-FSM } & \text { Wound-Field Flux Switching Machine } \\ \text { WPG } & \text { Wind Power Generation } \\ \text { UMP } & \text { Unbalanced Magnetic Pull } \\ \text { USD } & \text { US Dollars } \\ \text { VAWT } & \text { Vertical Axis Wind Turbine } \\ \text { WF } & \text { Wound-Field } \\ \text { WRIG } & \text { Wound Rotor Induction Generator } \\ \text { WRSG } & \text { Wound Rotor Synchronous Generator } \\ \text { WRSG } & \text { Wound Rotor Synchronous Motor } \\ 2-D & \text { Two-dimensional } \\ \text { 3-D } & \text { Three-dimensional }\end{array}$

\section{Latin}

$a b$ intra

from within

a priori

de facto

et al

from the former

in fact

and others

etc. (et cetera)

in situ

and the other things

inter alios

in the place

among others

per se

through itself

quid pro quo

what for what

sic

just so

vice versa

with position turned

viz.

namely

\section{French}

$v i s-\grave{a}-v i s$

in relation to

\section{International System of Units}

$\begin{array}{ll}\mathrm{A} & \text { Ampere } \\ \mathrm{deg} . & \text { Degree } \\ \mathrm{Hz} & \text { Hertz } \\ \mathrm{kA} & \text { Kiloampere } \\ \mathrm{kg} & \text { Kilogram } \\ \mathrm{kHz} & \text { Kilohertz } \\ \mathrm{kNm} & \text { Kilonewton-metre } \\ \mathrm{kW} & \text { Kilowatt } \\ \mathrm{m} & \text { Metre }\end{array}$




$\begin{array}{ll}\mathrm{mH} & \text { Millihenry } \\ \mathrm{mm} & \text { Millimetre } \\ \mathrm{ms} & \text { Milliseconds } \\ \mathrm{MW} & \text { Megawatts } \\ \mathrm{MWh} & \text { Megawatts-hours } \\ \mathrm{Nm} & \text { Newton-metre } \\ \mathrm{r} / \mathrm{min} & \text { Revolutions per minute } \\ \mathrm{s} & \text { Second } \\ \mathrm{T} & \text { Tesla } \\ \text { ton } & 1000 \mathrm{~kg} \\ \mathrm{~W} & \text { Watts } \\ \mathrm{Wb} & \text { Weber } \\ \mu \mathrm{H} & \text { Microhenry } \\ \Omega & \text { Ohm } \\ { }^{\circ} \mathrm{C} & \text { Degree Celsius } \\ { }_{0} \mathrm{o} & \text { Percent }\end{array}$

\section{Currencies}

$€$

$\mathrm{k} €$

$\$$

ZAR

European Euro

1000 Euros

American Dollars

South African Rands 


\section{ChAPter ONe}

\section{INTRODUCTION}

Globally, renewable energy is receiving broader attention among regions and countries. This is the case presented in a recent report, the Renewables 2016 Global Status Report in REN21 (2016) [1], wherein it is shown that wind power remained the leading source of new generating power capacity with a total global capacity of $433 \mathrm{GW}$ towards the end of 2015. It is equally noted in the same report that most top wind turbine manufacturers broke their own annual installation records, of which the average-size wind turbines are in the multi-megawatts (MW) range. Thus, based on increasing demand for industrial-scale power wind turbine systems, the need to reduce the cost of generation is becoming critical such that attention is now being directed towards the available wind generator drive concepts. In this chapter, the main focus is on different wind generator drivetrain technologies, as well as recent trends towards non-conventional wind generators. Already, the researcher had presented parts of the notes in this chapter as a conference paper in Akuru and Kamper (2014) [18], later published as a selected journal paper in Akuru and Kamper (2014) [97].

\subsection{Background}

In ancient times, the power from winds have been used to power ships, grain mills, water pumps and threshing machines, DNV/Risø: 2002 [2], Patel: 1999 [3] and Cao, Xie and Tan: 2012 [4]. The dearth of such applications of the wind power resource was propagated by the industrial revolution of the late nineteenth century. At the same time, between 1880 and 1900, the first successful experiments using wind to generate electricity were reported, Patel: 1999 [3] and Manwell, McGowan and Rogers: 2002 [5]. According to Manwell, McGowan and Rogers: 2002 [5], it was not until the 1970s when the OPEC oil crises heightened, did the use of wind to generate electricity began at a commercial scale. Since then, other factors such as technical advances, government support, climate change concerns, dwindling cost of energy ${ }^{1}$ (CoEs), improved reliability, etc., have continued to sustain the unprecedented development and commercial growth in wind power generation (WPG), as witnessed

\footnotetext{
1 According to a 2013 World Energy Council study, Salvatore et al (2013) [131], cost of energy (CoE) is the cost of producing electricity from each renewable energy technology, as well as the key drivers of project costs. These include the cost of financing as well as equipment, installation, operating, maintenance and fuel costs where applicable. The following four different cost matrices were presented, though the researcher will be referring to aspects in the first case throughout the proposed research:

i) Capital expenditure (CAPEX). This includes the total cost of developing and constructing a plant, excluding any grid-connection charges.

ii) Operating expenditure (OPEX). This is the total annual operating expenditure from the first year of a project's operation, given in per unit of installed capacity terms.

iii) Capacity factor. Also referred to as load factor, this is the ratio of the net megawatt hours of electricity generated in a given year to the electricity that could have been generated at continuous full-power operation, or 8,760 full hours.

iv) Levelised cost of electricity (LCOE). A USD/MWh value that represents the total lifecycle costs of producing a MWh of power using a specific technology.
} 
especially in the last two decades or so. The latest growth trend, from 2006-2016, for global wind power as recently reported in REN21 (2017) [6] is pictured in Fig. 1.1.

Wind turbines, as machines with rotating blades, convert kinetic energy from wind into electrical energy by the use of electrical machines, so-called wind generators. Thus, a wind generator converts mechanical energy, channeled through the turbine rotor, into electrical energy.

A wind turbine is made up of many subsystems among which are the turbine rotor blades, hub, nacelle, tower and foundation, to mention a few. The nacelle is where the generator and other drivetrain components such as the gearbox (for a geared system), mechanical couplings and brakes, and solid state converters (SSCs), are usually housed. Thus, the gearbox, generator and the SSC are critical components in wind turbines, which not only determine the size of a wind turbine nacelle, but to a great extent account for a reasonable amount of the total capital costs, IRENA: 2012 [8]. However, it is important to bear in mind that the costs of wind turbines, to a great extent, also depend on the country and site location where it is hosted, Polinder et al: 2013 [7] and Vandendael: 2013 [9]. Therefore, depending on the site location, a wind turbine can either be installed on land (onshore) or at sea (offshore). Offshore wind turbines provide higher wind speeds, which improve the wind turbine performance, as well as offer unrestricted sizes and sites, but are nonetheless prone to higher installation and maintenance costs.

It is also vital to note that a variety of wind turbine configurations exists such as horizontal axis wind turbine (HAWT) and vertical axis wind turbine (VAWT), based on the axis of rotation of the blades, with the former as the most dominant in industry. A further distinction can be made for HAWT structures with regards to the position of the turbine rotor blades - upwind rotor blades which are sandwiched between the tower and the side facing the direction of the wind or downwind rotor blades which are positioned after the tower and against the wind direction.

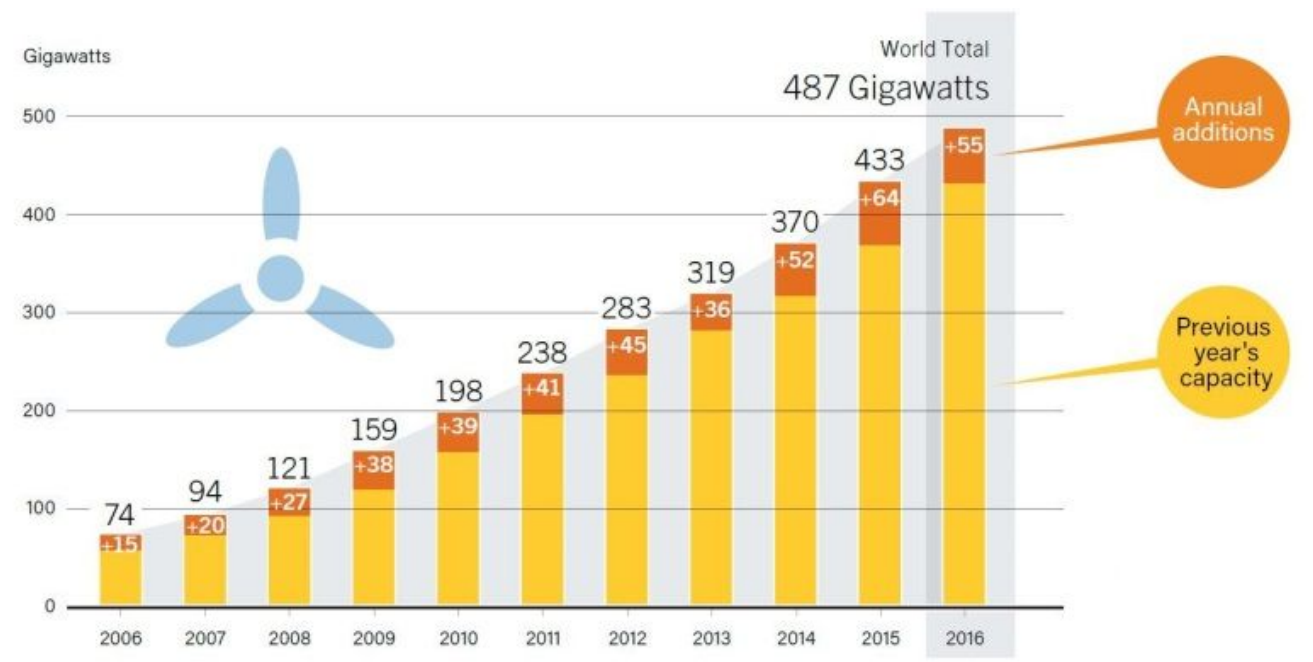

Fig. 1.1. Global wind power capacity and annual additions, 2006-2016 [6]. 
Furthermore, wind turbines can be categorised based on their grid interface-fixed-speed or variable speed. Fixed-speed wind turbines are so-called because, irrespective of the wind speed, the rotor speed of the wind generator is fixed as determined by the grid frequency. Conversely, variablespeed wind turbines, which are most commonly used today, are able to partially or fully isolate their wind generators from the grid, with the help of SSCs.

Yet, there are, equally, classifications of wind turbines according to how the rotor turbine shaft is connected to the generator shaft—-so-called geared and direct-drive systems. A geared drivetrain is when a gearbox is required to speed-up the incoming speed from the turbine rotor shaft entering the wind generator. Whereas if the turbine rotor shaft is connected directly to the generator shaft, a direct-drive configuration results. Further discussion on different wind turbine drivetrains is later provided in section 1.2.

Some other options for wind turbine classifications include but may not be limited to hub type (rigid, flexible, gimbaled or hinged blades), rigidity type (still or flexible), number of blades used (one, two, three or more), braking systems installed (stall, pitch, yaw or aerodynamic surfaces), and the rotor blades alignment mechanism (active or free yaw). More details on the last three paragraphs can be sourced from Patel: 1999 [3], Cao, Xie and Tan: 2012 [4], Manwell, McGowan and Rogers: 2002 [5] and Zhu and Hu: 2013 [10].

Over the last twenty five years, the sizes and power of wind turbines have grown significantly as shown in Fig. 1.2. This growth has prompted not only the size and cost, but also the overall market volume of wind generator design and manufacturing, wind generators acting as critical components in wind turbines, to increase dramatically, Polinder et al: 2013 [7]. With the growing wind turbine size, the choices for wind generator designs are mostly permanent magnet (PM) machines, viz., rareearth PMs. The use of such PM machines not only attract high cost as determined by the cost of PM materials, but also increases the costs of the associated drivetrain components e.g., SSCs. Note that, the availability and cost of rare-earth PMs are unpredictable due to apparent resource monopoly as stressed in Jahns (2017) [90]. Except for direct-drive systems, another component in the wind turbine drivetrain that is usually affected by size modulation, vis-à-vis costs, is notably, the gearbox system.

As a consequence, the need to reduce the cost of generation without compromising performance is considered as very important to research and development in WPG and as such, much focus is currently directed at both the available drivetrain (geared or direct-drive) and the generator (conventional or non-conventional), Cao, Xie and Tan: 2012 [4]. The next section is used to discuss the state-of-the-art in wind turbine drivetrains, by reviewing the current technology of different wind generator topologies. 


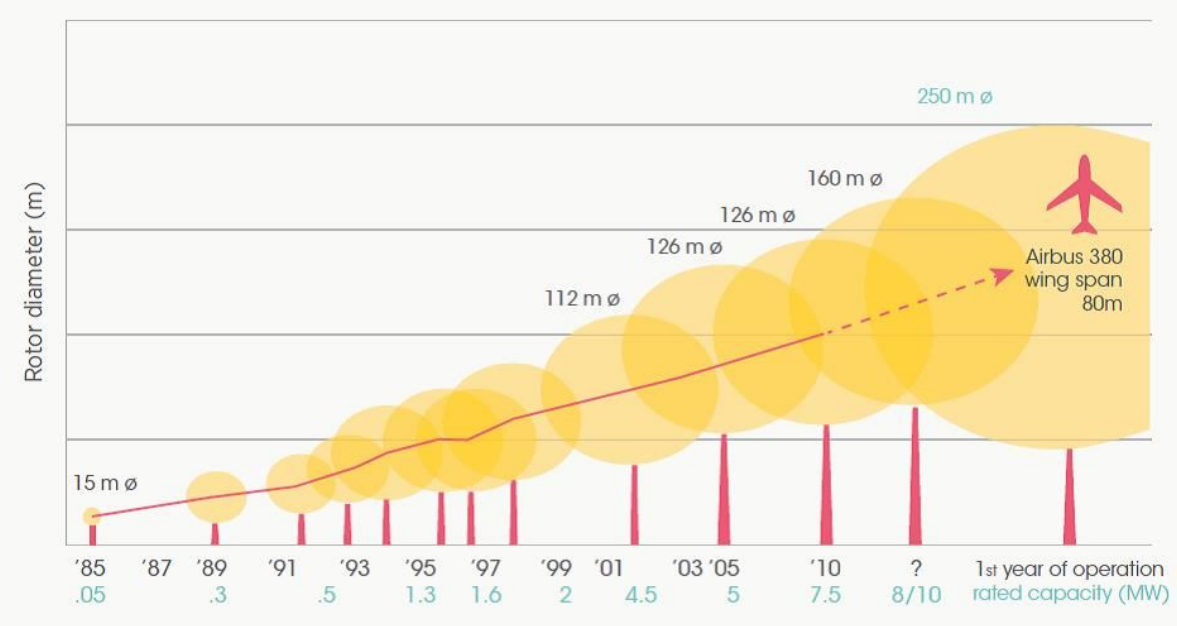

Fig. 1.2. Size and power increment in wind turbines since 1985 [8].

\subsection{Wind Turbine Drivetrain Technologies}

Given the recent push towards harnessing wind energy for industrial-scale power generation, the need to reduce the $\mathrm{CoE}$ is driving more and more researchers to concentrate on optimising the available drivetrains. The different known wind generator drive concepts are direct-drive (DD) a.k.a. lowspeed (LS) drives, medium-speed (MS) drives and high-speed (HS) drives, with the last two falling under the geared drivetrain category. Usually, the classification of wind generator drivetrains is made possible by the presence or absence of a gearbox. MS geared drives describe systems with a one- or less than a three-stage gearbox, while HS drivetrains are geared systems with a three- or more than a three-stage gearbox, Polinder et al: 2006 [11], Aydin: 2013 [12] and de Vries: 2012 [13].

It must be stressed in this study that, LS, MS and HS generator drivetrains should not be confused with low-speed, medium-speed or high-speed wind speed operations of wind turbines as implied in Gitano-Briggs (2012) [14]. Rather, the focus here is on the speed-limit at which the generator turns at steady-state, Aydin: 2013 [12]. Also, as corroborated in de Vries (2012) [13], the researcher is aware that some variants of LS and MS wind generator drive concepts exist in the industry which have single-stage and three-stage gearboxes, respectively, however the current study assumes a similar categorisation as done in Aydin (2013) [12], Tavner et al (2013) [22] and Li, Chen and Polinder (2006) [15], based on the following drivetrain specifications:

- LS is the absence of a gearbox system,

- MS is the presence of a one- or less than a three-stage gearbox, and

- HS is the presence of a three- or more than a three-stage gearbox.

Accordingly, Polinder et al (2006) [11] and de Vries (2012) [13] indicated that the most popu- 
lar among wind generator drivetrains are LS and HS systems, with over $80 \%$ existence attributed to the latter. No doubt, HS drivetrains offers minimum generator mass as opposed to LS systems, although such is achieved by sacrificing large generator mass for a bigger gearbox size cum costs. As a result of enlarged gearbox size, HS systems are not only prone to high installation costs but are also expensive to maintain due to increased reliability issues, Polinder et al: 2013 [7], Zhu and Hu: 2013 [10] and Ragheb and Ragheb: 2011 [16]. However, a report by ReliaWind Project (2011) [17] suggests otherwise, claiming that the high frequency attributed to gearbox failures in wind turbines, based on recent assessments, is debatable.

Meanwhile, LS systems are designed without gearbox and as a result, were initially favoured as the future of wind turbines, Zhu and $\mathrm{Hu}: 2013$ [10]. But nowadays, it is now known that LS systems result in the largest and heaviest wind generators, especially at multi-MW power ratings, Aydin: 2013 [12]. Moreover, because wind generators designed for LS drivetrains are mostly manufactured using rare-earth PMs as revealed in Zhu and Hu (2013) [10], Polinder et al (2006) [11], Aydin (2013) [12], Tavner et al (2013) [22] and de Vries: 2012 [19], their manufacturing costs also increase dramatically. This is due to their operating 'low-speed' drivetrain, which is responsible for a disproportionate increase in size of the wind turbine if a constant power capture corresponding to, for example, a geared HS system is to be maintained. To further explain this, consider the mechanical input power of a wind turbine which is given as

$$
P_{m}=\frac{1}{2} \rho_{a i r} C_{p}\left(\lambda_{v}, \theta_{p}\right) A_{W} v^{3}
$$

where $\rho_{\text {air }}$ is the air fluid density, $C_{p}$ is the aerodynamic efficiency being a function of the tip speed ratio $\left(\lambda_{v}\right)$ and blade pitch angle $\left(\theta_{p}\right), A_{W}$ is the wind turbine rotor swept area and $v$ is the wind speed. Note that, the size of a wind generator is a tradeoff between its torque and speed. Hence, at a fairly constant wind turbine power, observe that a lower generator steady-state speed implies a larger size rotor blade swept area, vis-⿳亠丷- -vis wind turbine, to satisfy a higher mechanical driving torque.

On the other hand, the main advantage of LS drivetrains is that they are gearless; therefore, they present no worries on gearbox maintenance issues, Polinder et al: 2006 [11]. In addition, LS drivetrains are nominated for best efficiency while operating at partial loads, Schmidt and Vath: 2012 [20] and Matveev: 2011 [21].

In-between HS and LS drivetrains are geared MS drivetrains. Geared MS drives first appeared in the mid-1990s when it was first developed by Multibrid, then known as hybrid drives because it was considered an intermediate between HS and LS drives, Polinder et al: 2006 [11] and de Vries: 2012 [19]. Among these three major drivetrains, geared MS drives can provide the lowest CoE, low- 
est maintenance and highest efficiency, while increasingly having an established industry footprint, Polinder et al: 2006 [11], de Vries: 2012 [13], Schmidt and Vath: 2012 [20], Tavner et al: 2013 [22], Coultate: 2011 [23] and Vath: 2012 [24].

Schmidt and Vath (2012) [20] were able to show that MS drive concepts, which come with 1or 2-stage gearboxes together with considerable high-pole generators, can lead to greater annual energy yield per generator costs at average wind speeds. They compared several wind turbine drivetrain concepts at full-load using PM generators. Consequently, they attributed the lowest CoE and highest efficiency to the MS drivetrain as illustrated in Fig. 1.3 and Fig. 1.4, respectively. Their findings which portray MS drivetrain as the drivetrain with the lowest CoE have been supported in other studies such as Cao, Xie and Tan (2012) [4] for 1-stage DFIG and Bang et al (2008) [29] for 1-stage PMSG.
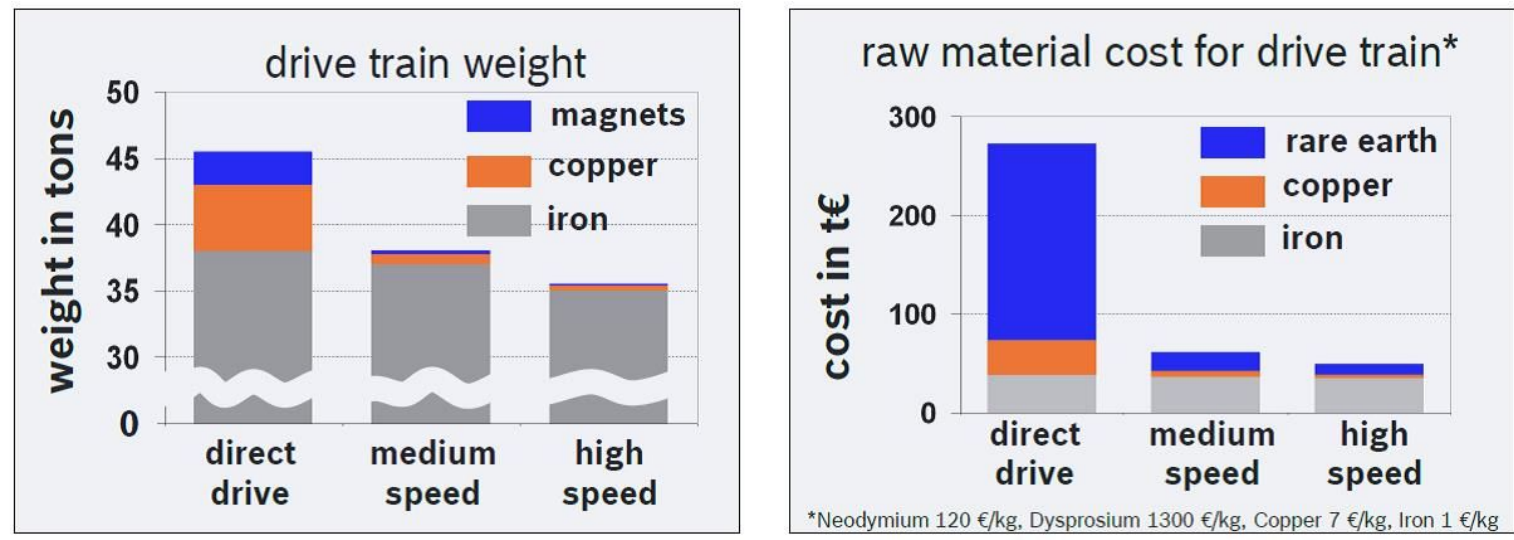

low amount of raw materials

low drive train material costs

Fig. 1.3. Comparison in terms of mass and cost of energy of PMSG evaluated for different drivetrains at $4 \mathrm{MW}[20]$.

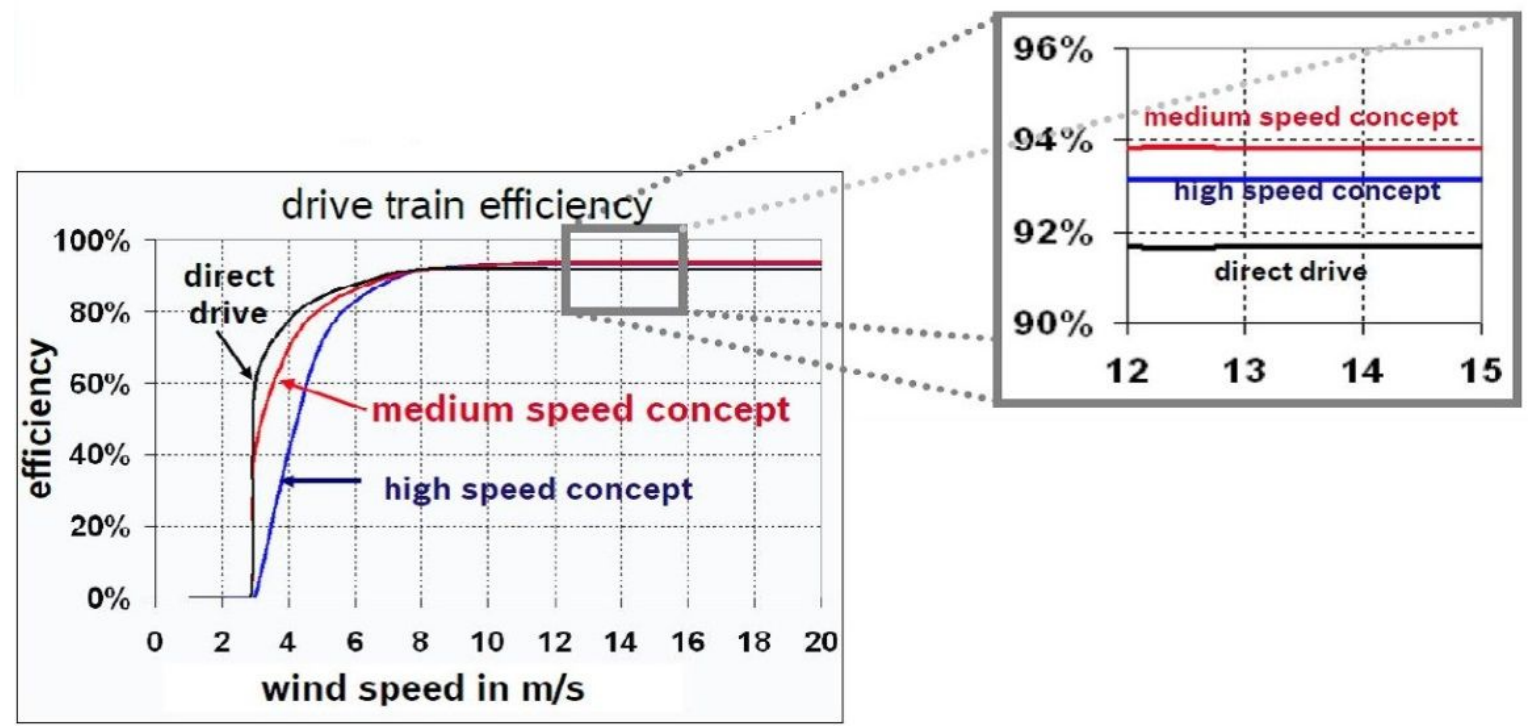

Fig. 1.4. Efficiency of PMSG evaluated for different drivetrains at 4 MW [20]. 
Table 1.1. Comparison of the different drivetrain concepts

\begin{tabular}{c|c|c|c}
\hline Parameter & HS & MS & LS \\
\hline \hline Speed margin & $600-2000 \mathrm{r} / \mathrm{min}$ & $40-600 \mathrm{r} / \mathrm{min}$ & $4-35 \mathrm{r} / \mathrm{min}^{2}$ \\
Mass & Lightest & Intermediate & Heaviest \\
Size & Smallest & Intermediate & Largest \\
Gearbox presence & Yes $\left(3 \mathrm{G}^{3}\right)$ & Yes $(1 \mathrm{G} / 2 \mathrm{G})$ & Absent \\
Generator type & $\mathrm{IG} / \mathrm{SG}^{4}$ & IG/SG & SG \\
Mechanical losses & High & Intermediate & Lowest \\
Electrical losses & Lowest & Intermediate & Highest \\
Cost $^{5}$ & Gearbox & Intermediate & Generator \\
\hline
\end{tabular}

To this end, some specific benefits of MS drivetrains include, but are not limited to the following:

i) Low structural, capital and operational costs: utilisation of simple 1- or 2-stage gearbox leading to smaller generator and drivetrain mass, and comparable cost of energy;

ii) Improved reliability and improved efficiency due to the absence of HS gearboxes known to cause failures, and

iii) Compact size of wind generators which lead to lower top head mass for easier logistics, as well as reduction in tower materials required in the use of moderate-sized nacelle.

In summary, the different drivetrain characteristics as provided in Table 1.1 show that the MS drivetrain is able to yield better compromise compared to LS and HS drivetrain concepts. An important point to also note from Table 1.1 is the speed-range as shown for the different drivetrains ${ }^{6}$. No doubt, drivetrain designs have meaningful impact on the performance of wind turbines. Consequently, to leverage on the salient advantages researched on geared MS drives, the researcher begins to propose in this study that, with the appropriate generator, the possibility to reduce the overall system costs, with minimum compromise on the drivetrain performance, is not in doubt. To this end, the next section is devoted to such an inquiry.

\subsection{Current Wind Generator Topologies}

As revealed in studies by Cao, Xie and Tan (2012) [4], Zhu and $\mathrm{Hu}$ (2013) [10] and Polinder et al (2006) [11], there is yet to be a consensus on the best wind generator machine. Since geared MS wind generator drives is the least developed in terms of wind generator drivetrain technologies, a

2 Also depends on operating power level.

$3 \mathrm{G}$ represents the gearbox stage.

$4 \mathrm{IG}=$ induction generator, $\mathrm{SG}=$ synchronous generator

5 This implies the component with the dominant cost.

6 In the literature, this range is highly debatable for the generality of electric machines, whereby MS is sometimes quoted to reach $4000-8000 \mathrm{r} / \mathrm{min}$ according to Kolehmainen and Ikaheimo (2008) [132], because it appears there are no established standards. The controversy also affects wind turbine manufacturers because some have used 1-, 2-, and even 3-stage gearbox system(s) to design wind generators in the MS range, de Vries: 2012 [13]. Although in this study, the researcher prefers to maintain 1- or 2-stage as the standard for the MS gearboxes, based on a gear ratio of between 10 and 40 , to describe a typical medium-speed wind generator. For instance, in a two-stage gearbox, the maximum achievable step-up gear ratio is usually fixed at 1:40. 
most suitable wind generator type is not yet ascertained. To this end, this section is dedicated to understudy the general trend in wind generator design.

\subsubsection{Conventional Wind Generators}

Conventional wind generators are wind generators that are designed and manufactured from traditional electrical machine concepts. These set of wind generators have been in existence from the onset of modern wind turbines. In Cao, Xie and Tan (2012) [4], the three main types of traditional wind generators are presented as: direct current (DC), alternating current (AC) synchronous and AC asynchronous generators. Asynchronous AC generators are usually squirrel cage rotor induction generators (SCIGs), wound rotor IGs (WRIGs) and doubly-fed IGs, so-called DFIGs, whereas synchronous generators include PMSGs and WRSGs, sometimes ascribed as electrical excited SGs (EESGs).

Information on DC generators, its theory, design and operation are well established, even by a simple online Google search. In undertaking such online research, some conceptual terms like fields, armature, stator, rotor, brushes, commutators, etc., which are general terminologies in the design of electrical machines, are displayed. In reality, DC machines acting as wind generators are not common. In fact, Cao, Xie and Tan (2012) [4] purportedly reported that when they do exist as wind generators, such wind turbines are usually in low power installations. One reason for their unpopular wind generator potentials might be attributed to its high maintenance costs due to inherent presence of commutators and brushes.

In Polinder et al's (2006) [11] study, where some current and future wind turbine generator systems were discussed, DC generator topologies, actually, did not feature. A similar outcome is observed in Li, Chen and Polinder (2006) [15]. Needless to say that prospects of DC generators for WPG is bleak, and with almost no account of its existence among wind generator manufacturers as appraised in studies by Matveev (2011) [21] and Ragheb (2014) [25]. However, some studies do abound where DC generator have been investigated for WPG, but they are usually operated in LS drivetrains, which unfortunately may further complicate both the manufacturing and maintenance challenges of the generator, Cao, Xie and Tan: 2012 [4] and Madani: 2011 [26].

On the other hand, induction generators are so popular for wind generator designs such that they are currently ranked as the highest used machines in the industry, Cao, Xie and Tan: 2012 [4], de Vries (2012) [13] and Zou: 2015: [27]. In particular, Zou (2015) [27] discussed the nitty gritty of using IGs for wind power systems, which, for the sake of avoiding redundant duplications, would not be comprehensively rehashed here. To this end, the common drivetrain topologies of IGs as used for WPG have been reproduced as shown in Fig. 1.5. 
The SCIG system is dubbed as "cheap" in Polinder, et al (2013) [7], but it is usually operated at fixed speed, thereby limiting the power capture, among other issues. In the same vein, Cao, Xie and Tan (2012) [4] wrote about SCIGs that they are "simple, reliable, inexpensive and well developed... but they draw reactive power from the grid and thus some form of reactive power compensation is needed". Furthermore, because SCIGs are grid-tied, it means that they do not permit adjustment of their output voltage, and they also experience limitations such as audible noise, low efficiency and high maintenance costs mainly due to its operation in multi-stage geared wind generator drivetrains, de Vries: 2012 [13], Ragheb: 2014 [25] and Zou: 2015 [27]. But to give credit to the earliest developers of modern wind turbines, SCIGs are distinguished in wind generators' hall of fame as the wind generators used in the popular Danish concept which ranged up to $1.5 \mathrm{MW}$ between 1980 and 1990.

On the other hand, WRIGs are slightly better than SCIGS in that they can be operated at variable speeds via a rotor resistance slip control, but in a very limited range. Today, DFIGs are the reigning superpower, not only among IGs, but among wind turbine generators generally. They have been designed to reach a capacity of $5 \mathrm{MW}$, according to Zhu and $\mathrm{Hu}$ (2013) [10]. The advantage of DFIGs is that they require only some percentage (20-34\%) of the generator nominal power to devise the ratings of the SSCs, which assist them to provide a wider range of speed variation compared to WRIGs, Zhu and Hu: 2013 [10] and de Vries: 2012 [13]. The known disadvantages of DFIGs are: presence of slip-rings and the use of a three-stage gearbox, as well as grid interconnection challenges as detailed in Polinder et al (2006) [7] and $\mathrm{Zhu}$ and $\mathrm{Hu}$ (2013) [10].
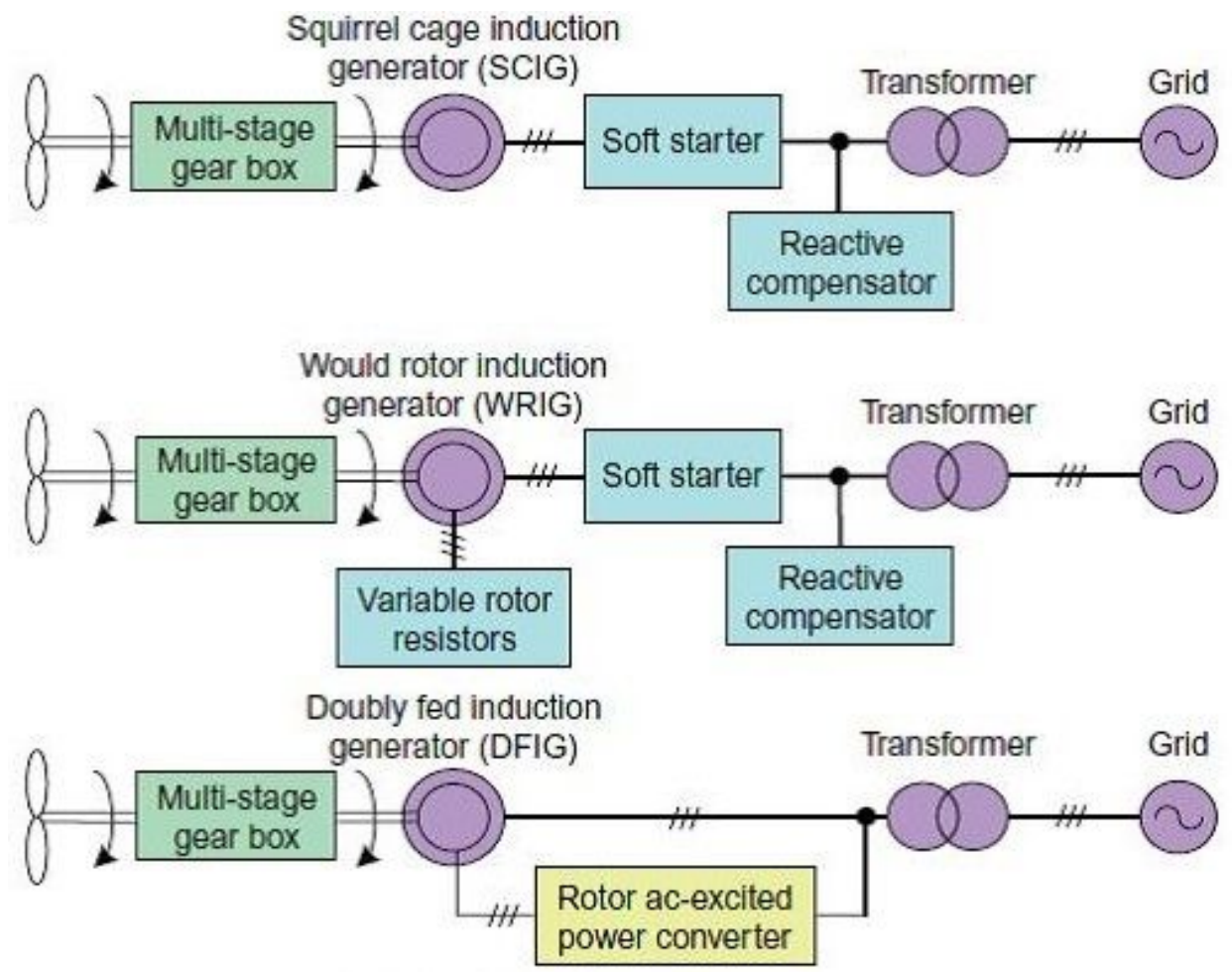

Fig. 1.5. Different wind generator drivetrains designed with induction generators [10]. 
Now, enter in the synchronous generators (SGs). SGs are growing in popularity today because, unlike DFIGs, they are fully decoupled from the grid with a fully-rated power converter (FPC), thus facilitating a wider variable speed range with superior grid compliance, de Vries: 2012 [13]. In some cases, they are operated as direct-drive systems, thus improving their drivetrain reliability, Dubois: 2004 [28]. However, Bang et al (2008) [29] and Semken et al (2012) [30], both agree that the main challenge with SGs, if designed for gearless drives, is a resulting large volume, which increases the generator costs, especially when rare-earth PMs are used.

As a matter of fact, PMSGs coupled with very high PM volumes pose added risk of PM demagnetisation due to poor thermal dissipation, in addition with associated motive forces on their rotor-housed PMs, Madani: 2011 [26], Chen et al: 2015 [31] and Sjökvist: 2014 [32]. Also, their fields, based on PMs, are not controllable. However, because PMSGs are self-excited, the problems of brushes and slip rings qualify them as very robust electrical machine candidates.

Next are the WRSGs or EESGs, with wound-fields (WFs) replacing PMs, which makes them advantageous in terms their ability to produce reactive power and regulate output voltage by the regulation of the field current, Madani: 2011 [26] and Zhu and Hu: 2013 [10]. Nevertheless, they experience maintenance and efficiency issues. The different drivetrain topologies so far discussed for SGs are summarised as shown in Fig. 1.6.
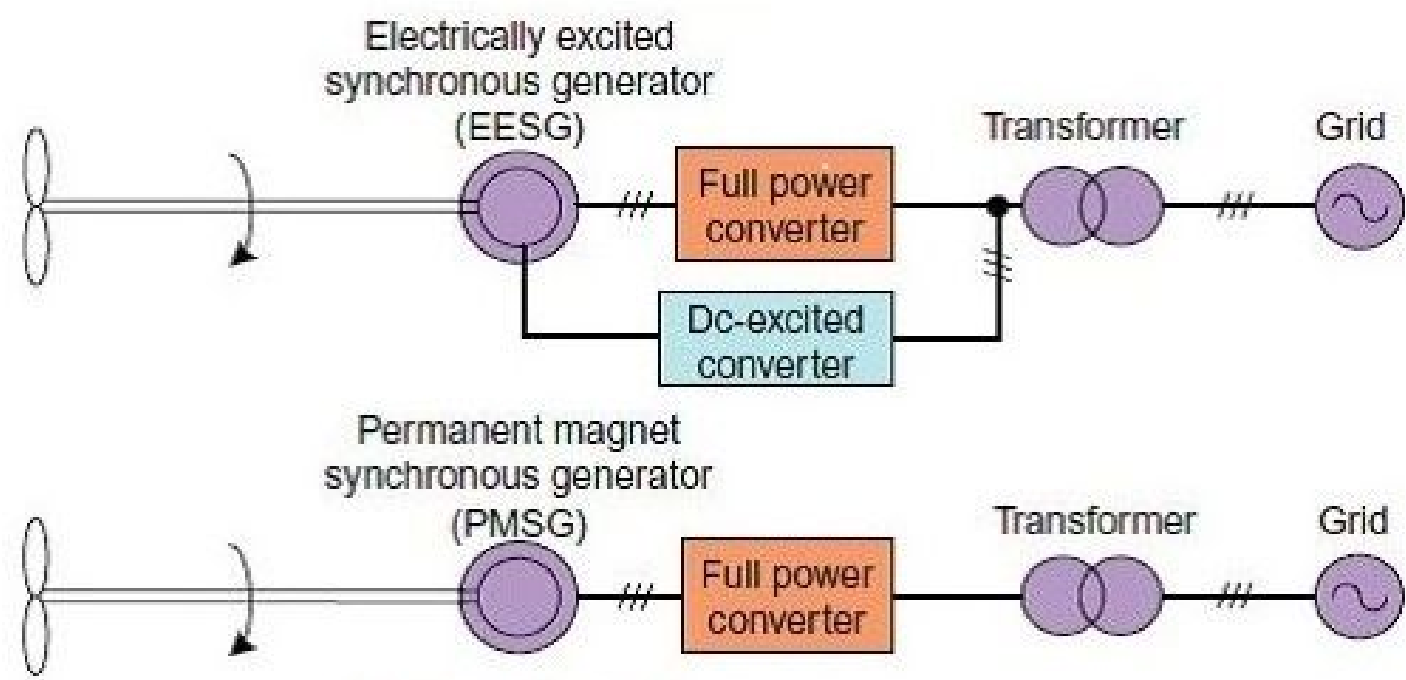

Permanent magnet

synchronous generator

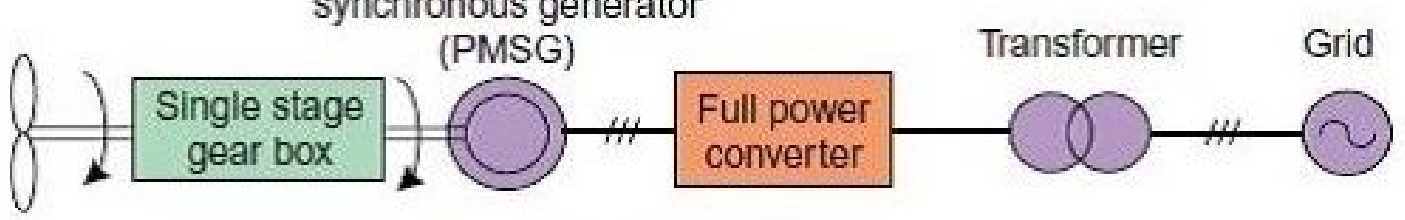

Permanent magnet

synchronous generator

Fig. 1.6. Wind generator drivetrains for different synchronous generator topologies [10]. 
Table 1.2. Quantitative comparison of three major wind generators ${ }^{7}[4]$

\begin{tabular}{c|c|c|c|c|c}
\hline \multirow{2}{*}{ Parameter } & \multicolumn{2}{|c|}{ DFIG } & \multicolumn{2}{c}{ Synchronous generators } \\
\cline { 2 - 6 } & \multicolumn{2}{|c|}{ geared } & PM & EE & PM \\
\cline { 2 - 6 } & - & - & direct drive \\
\cline { 2 - 6 } & 1-stage & 3-stage & 1-stage & - & - \\
\hline \hline Airgap diameter (m) & 3.6 & 0.84 & 3.6 & 5 & 5 \\
Stack length (m) & 0.6 & 0.75 & 0.4 & 1.2 & 1.2 \\
Iron weight (ton) & 8.65 & 4.03 & 4.37 & 32.5 & 18.1 \\
Copper weight (ton) & 2.72 & 1.21 & 1.33 & 12.6 & 4.3 \\
PM weight (ton) & & & 0.41 & & 1.7 \\
Generator active material cost $(\mathrm{k} €)$ & 67 & 30 & 43 & 287 & 162 \\
Gearbox cost (k€) & 120 & 220 & 120 & & \\
Converter cost (k€) & 40 & 40 & 120 & 120 & 120 \\
Generator construction cost $(\mathrm{k} €)$ & 60 & 30 & 50 & 160 & 150 \\
Total generator system cost $(\mathrm{k} €)$ & 287 & 320 & 333 & 567 & 432 \\
Annual electricity yield $(\mathrm{MWh})$ & 7760 & 7690 & 7700 & 7740 & 7890 \\
Yield/total cost (kWh/k€) & 4.22 & 4.11 & 4.09 & 3.67 & 3.98 \\
\hline
\end{tabular}

A detailed quantitative performance comparison of the traditional wind generators as reported in Cao, Xie and Tan (2012) [4] is reproduced in Table 1.2. Based on conclusions drawn in section 1.2 , it is not surprising to note that, among other things, the 1-stage geared (MS) DFIG present an obvious advantage in terms of energy yield per total cost compared to the other wind generator drivetrains.

In summary, most conventional wind generators employ a multi-stage gearbox together with commutator brushes, expensive rare-earth PMs, and slip-rings in the rotors of e.g., DC wound-field configurations. Meanwhile, the use of PMSGs poses two major problems, high cost of PMs and demagnetisation risks. Hence, for multi-MW systems, these problems are necessarily scaled up. However, there are emerging brushless wind generators today, with some having stator-active and robust rotor qualities. These brushless machines are very attractive because they lack the use of brushes in all ramifications, and their hotspots are easily exposed to cooling schemes. Thus, the next section will be used to discuss the generality on the state-of-the-art of non-conventional, viz., brushless wind generators and their potentials for the proposed geared MS wind generator drives.

\subsubsection{Non-Conventional Wind Generators}

Apparently, the most established brushless wind generators are the SCIGs, Li, Chen and Polinder: 2006 [15]. This constant speed system is already discussed in subsection 1.3.1. They were eventually replaced by DFIGs, which though are not entirely brushless in nature, but are able to produce higher energy yield due to a wider operating speed range. 
Also, PMSGs as discussed in the preceding subsection can be considered as brushless; but because the PMs are positioned internally within the rotating member, they become vulnerable to temperature rise and certain motive forces, which can result in demagnetisation and mechanical stress, respectively. Besides, the high cost of high-energy PMs is another major disadvantage of PMSGs in spite of remarkable benefits such as potentially reduced generator active mass, as well as capabilities for high power density and efficiency, Cao, Xie and Tan: 2012 [4] and Madani: 2011 [26].

Switched reluctance generators (SRGs) are next in line to be considered as brushless wind generators, and the first to be highlighted as stator-active machines with a robust rotor structure. In addition, because they have both salient rotors and stators, they are also called double salient machines. SRGs are simple to construct (bodily), mechanically robust and can withstand high temperature conditions, but they produce loud acoustic noise as attributed in Mademlis and Kioskeridis (2005) [33] and Ahmad (2010) [34]. Besides, they have a complicated control strategy in that, "they obtain their excitation from the same voltage bus that it generates power to", citing Husain, Radun and Nairus (2002) [35]. Karthikeyan (2009) [36] suggested that the suitable drivetrain topology for SRGs are LS drivetrains; this, of course, would make them very bulky and expensive for WPG systems.

In Potgieter's (2014) [85] thesis, some details on the emergence of non-conventional wind generator concepts is provided. Eventually, one such candidates, the slip synchronous permanent magnet generator (SS-PMG) design, was preferred for his study because, according to him, it has improved simplicity, robustness, reliability and grid compatibility compared to most conventional wind generator systems. However, the researcher is aware that SS-PMG's are usually configured for fixed speed wind turbine systems, coupled with a complex and bulky architecture, which is acerbated by the assembling of two direct-drive generators, among others.

Wang and Gerber (2014) [86] investigated on the use of magnetic geared PM (MGPM) machines for wind generator drives. Their findings, among other things, show that such generator topologies have potentially higher efficiency, better thermal management and higher reliability compared to conventional geared wind generator drives. However, the complexity and PM-reliance of such systems is again bound to increase their manufacturing costs.

Yet, there are new and emerging stator-active brushless wind generator concepts such as the PM flux switching machine (PM-FSM) studied in Ojeda et al (2012) [37]. PM-FSMs are not alone in this category, as others such as PM flux-reversal machines (PM-FRMs) and the PM doubly salient machines (PM-DSMs) have been similarly designed for wind energy applications in Saou, Zaïm and Alitouche (2009) [38] and Fan, Chau and Cheng (2006) [39], respectively. These categories of brushless machines have similar features which distinguish them from other machines such as, a robust 
rotor structure, ease for thermal cooling due to stator-mounted components and the capacity to deploy less copper usage due to a concentrated winding configuration, among others.

Interestingly, PM-FSM is being resurrected after it was initially invented by Rauch and Johnson (1955) [40], which unlike the PM-DSM was first devised by Liao, Laing and Lipo (1995) [41] and the PM-FRM by Boldea, Serban and Babau (1996) [42]. These brushless machines tend to site their active components (be it PM blocks or DC coils), as well as the phase windings, mainly in their stators as similarly contrived in SRGs. Thus, they result in less cumbersome rotor structures as depicted in Fig. 1.7.

Different from SRGs, their field sources are physically distinguishable by either PMs or field coils in the stators, making their design less complicated. Considering that cooling measures are also easier to deploy, they are further preferred for HS fault-tolerant applications. Also, they have very good flux weakening ${ }^{8}$ capabilities, which is mainly beneficial in automotive applications, Fasolo, Alberti and Bianchi: 2014 [69], Zhu, Shen and Howe: 2006 [133] and Štumberger et al: 2006 [134].

An overview on these stator-active PM machines is already provided in Hua et al (1999) [43] and Cheng et al (2011) [44], hence the researcher is not going to go on and on about it. In Hua et al (1999) [43], it was simply about PM-FSMs - their features and what makes them interesting for a number of application needs; their different excitation modes such as PM-FSMs (only PMs), WFFSMs (only wound-fields) and HE-FSMs for hybrid-excited systems (both PMs and wound-fields); their electromagnetic performance; as well as their different stator and rotor topologies.

In Cheng et al (2011) [44], the discussion was generally on all three stator-active machines, with focus on their concepts, operating principles, machine topologies, electromagnetic performance, etc. They were able to provide a summary on the advantages and disadvantages of all three machines, based on a qualitative comparison, replicated here in Table 1.3 for emphasis.

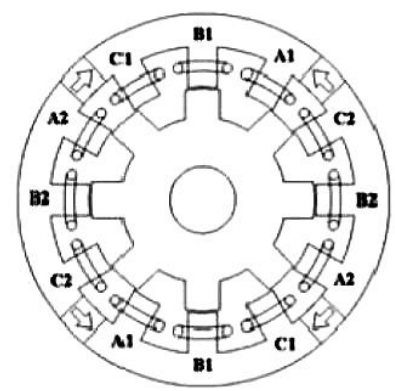

(a)

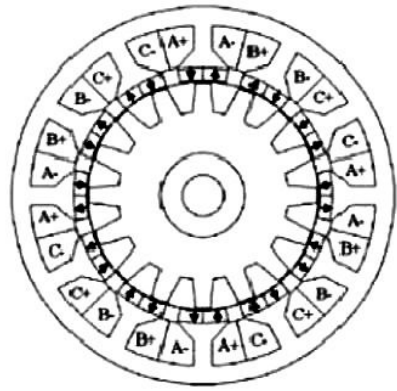

(b)

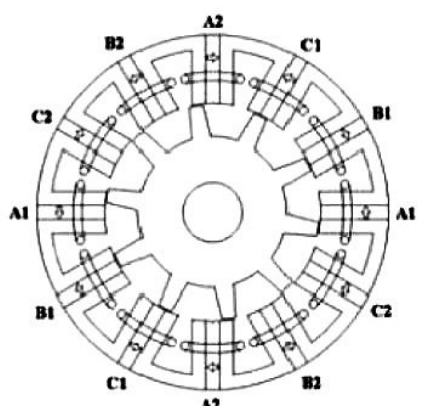

(c)

Fig. 1.7. Cross-sections of stator-active machines ${ }^{9}$ : (a) PM-DSM, (b) PM-FRM, and (c) PM-FSM.

\footnotetext{
${ }^{8}$ Flux weakening is a control strategy usually employed to extend the operating speed range of electric drives, while maintaining constant power. In cases of coils e.g., WRSGs, it is achieved by controlling the field current magnitude to adjust the back-EMF which is speed dependent, while in other cases, e.g., PM-FSMs, it is characteristically achieved by forging a compromise between the mechanical torque and output power, when the speed is adjusted.

9 Source: SAE 2011 Powertrain Electric Motors Symposium-Indianapolis.
} 
Table 1.3. Comparison of different novel stator-PM machines [44]

\begin{tabular}{c|c|c|c}
\hline Design issue & PM-DSM & PM-FSM & PM-FRM \\
\hline \hline Phase flux & Unipolar & Bipolar & Bipolar \\
Energy conversion loop & 1st and 2nd quadrants & All four quadrants & All four quadrants \\
PMs location & Stator back iron & Sandwiched by stator teeth & Stator teeth surface \\
PMs consumption & Low & High & Medium \\
EMF waveform & Trapezoidal & Sinusoidal & Trapezoidal \\
Cogging torque & Low & High & Medium \\
Torque density & Low & High & Medium \\
Control mode & BLDC & BLAC & BLDC \\
\hline
\end{tabular}

It is clear to see from Table 1.3 that, PM-FSM provides the best qualities in terms of torque density and bipolar flux linkages among the rest, thus making it a promising candidate for the proposed geared MS wind generator drives. Little wonder, therefore, why Cheng et al (2011) [44] concluded their appraisal with the prediction of a bright future for PM-FSMs in areas such robotics, automobiles, wind energy drives, to mention a few.

Just like in Cheng et al (2011) [44], Zhang et al (2009) [45] also embarked on a comparative study on all three stator-active PM machines but this time, it was based on the formulation of their respective general power equations. After establishing the equations, which were used for the comparison, they equally confirmed in their findings that the PM-FSM option possesses the highest power density among the three machines.

But, as highlighted in Table 1.3, a major setback in PM-FSMs is their characteristically high cogging torque and torque ripple, which is due to its double salient structure. Even when compared to other conventional machines, they do not fare any better in this regard, according to the studies in Hua et al (2008) [46] and Pang et al (2007) [47]. Shao et al [103] provided in tabular form, a comparison of stator-PM machines e.g., PM-FSMs, with typical rotor-PM machines, reproduced herein as Table 1.4. Clearly, it can be further seen that the airgap field harmonics in PM-FSMs is very high, another factor usually attributed to the effects of high cogging torque and torque ripple in PM-FSMs. To this end, numerous studies have been initiated in an attempt to address the problem of high cogging torque and torque ripple in FSMs, with some reasonable success already established.

For instance, Sikder, Husain and Ouyang (2015) [48] used flanges together with rotor pole shaping to reduce cogging torque, while Wang, Wang and Jung (2012) [49] employed rotor teeth notching schemes but at a slight detriment to the average torque. Also, Xu et al (2011) [50] applied several techniques such as rotor pole-pairing, rotor pole-notching, rotor pole-chamfering and rotor pole-skewing, just to ensure that they topple the problem of cogging torque in PM-FSMs, once and for all. On the other hand, $\mathrm{Xu}$ (2014) [51] proposed a novel decoupling model-based predictive cur- 
rent control algorithm to diffuse torque ripple effects as against popular techniques which are based on electromagnetic optimal design, whereas Hwang et al (2016) [52] employed the winding function theory on the back-EMF and MMF airgap harmonics in their attempt to diminish torque ripple in PM-FSMs, to mention a few.

Again, another debilitating problem in PM-FSMs is their high PM utilization as also implied in Table 1.3, which can greatly increase their cost, when designed using very expensive PM materials like rare-earth PMs. One way to tackle this problem is by employing rare-earth-free materials like ferrite PMs or wound-fields. The feasibility of using rare-earth-free excitation sources is suggested in the light of the following:

- PM-FSMs have flux focusing ${ }^{10}$ capabilities as mentioned in Hua et al (2005) [53], which can be exploited by the use of lower performing rare-earth-free materials such as ferrite PMs or wound-fields, leading to a more economical design at reasonable performance constraints.

- Although with higher demagnetisation risks compared to rare-earth PMs, ferrite PMs used in PM-FSMs are to come without any fear of demagnetisation during normal operation, due to a special magnetic circuitry produced in PM-FSMs, which constructively align the field source MMF and the stator current MMF, Amara et al: 2005 [54] and McFarland, Jahns and ElRefaie: 2014 [55].

- PM-FSMs are low maintenance machines due to their brushless nature. Hence, the problem of brushes and slip rings, in the case of their wound-field designs, are totally eliminated.

Table 1.4. Comparison between PM-FSM and rotor-PM machines [103]

\begin{tabular}{c|c|c}
\hline Design issue & PM-FSM & Rotor-PM \\
\hline \hline Airgap field harmonics & High & Low \\
Back-EMF harmonics & Low & High \\
Simple and robust rotor & Yes & No \\
Simple stator & No & Yes \\
PM temperature management & Easy & Difficult \\
Copper slot area & Small & Large \\
Copper loss & High & Low \\
Saturation & Heavy & Light \\
Overload capability & Poor & Good \\
Stator outer flux-linkage & Yes & No \\
PM volume & High & Low \\
\hline
\end{tabular}

\footnotetext{
${ }^{10}$ Due to doubly salient structure and smaller overlapping areas between the stator and rotor teeth, as well as the position of field excitation (usually PM) sandwiched in-between ' $U$ '-core laminations in the stator of FSMs, the flux linking the rotor and the stator teeth tend to saturate, resulting in high airgap flux density. In Kim et al (2005) [135], it is called spoke configuration which is meant to mount magnets in PM machines such that magnets with poles of the same kind are placed facing each other, with alternate magnet poles in-between. This allows the flux from a large area of $\mathrm{PM}$ to be directed into the rotor pole, that is why it is known as flux focusing or flux concentration.
} 
- PM-FSMs being stator-active machines, does present an easier option for cooling of the field sources in instances with high operating temperature concerns.

Considering these important qualities which have triggered enormous traffic in the study of PM-FSMs, the researcher became quizzical and proceeded to perform an assessment on the state-ofthe-art on PM-FSMs in relation to wind generator drives. The next section is thus dedicated to this inquiry and the outcome thereof. The operating principle of the flux switching machine, which is yet to be discussed, should the reader be interested, is referred to in Appendix A1.

\subsubsection{State-of-the-art on Flux Switching Machine Wind Generator Drives}

Just like in typical PMSGs, PM-FSMs appear also in various designs such as axial flux (AFM, with disc type rotors), transverse flux (TFM, with pancake or disc type rotors) or radial flux machines (RFM, with cylindrical rotors). The thing about RFMs is that the flux direction is perpendicular to the axis of the airgap, whereas AFMs have their airgaps parallel to the flux direction. TFMs are machines that share both qualities of RFMs and AFMs - flux lines like in RFMs and rotors like in AFMs. They eventually achieve flux lines that lie in planes transverse to the direction of motion, usually with a torus or ring-shaped windings, Madani: 2011 [26], Bang: 2010 [56] and Nipp: 1999 [80].

The original PM-FSM design by Rauch and Johnson (1955) [40] is of the RFM structure; a $50000 \mathrm{r} / \mathrm{min}, 3 \mathrm{~kW}, 250 \mathrm{~V}$, single-phase, 4-stator poles/6 rotor poles flux-switch alternator as shown in Fig. 1.8. Subsequently, ensuing studies were more than often patterned according to the RFM design demonstrated over the years as in: Hoang, Ben Ahmed and Lucidarme (1997) [58], Pollock and Wallace (1999) [59], Pollock and Brackley (2003) [60], Amara et al (2005) [54], Hua et al (2005) [53], Hua et al (2006) [61], Pang et al (2007) [47], Hua et al (2008) [46], Zhu et al (2008) [62], Thomas et al (2008) [63], Chen et al (2010) [64], Chen and Zhu (2010) [65], Chen and Nilssen and Nysveen (2010) [66], Tang et al (2012) [67], Somesan and Viorel (2013) [68], Fasolo, Alberti and Bianchi (2014) [69], Zhou and Zhu (2014) [70], McFarland, Jahns and El-Rafaie (2014) [55], Raminosoa et al (2015) [71], Sulaiman et al (2016) [72] and Hwang et al (2016) [52], etc.

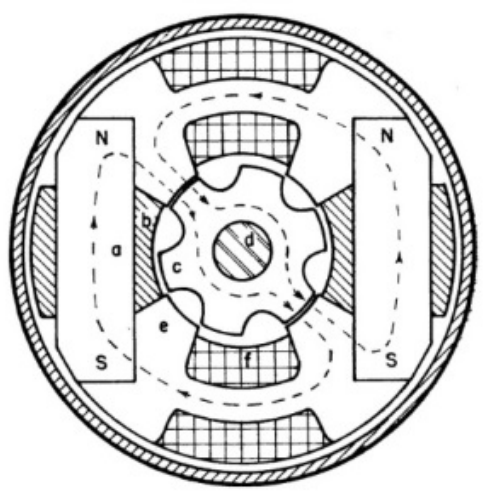

Fig. 1.8. Cross-section of the flux-switch alternator proposed in [40]. 
But, it has to be mentioned that most of these studies are predominantly in terms of high-speed traction drives, with exceptions based on studies by Amara et al (2005) [54] and Thomas et al (2008) [63] for aerospace applications, Sulaiman et al (2016) [72] for an electric boat, Chen, Nilssen and Nysveen (2010) [66] for downhole applications, and Hwang et al (2016) [52] for an elevator door.

In recent times, AFM and TFM types of PM-FSMs have been investigated mostly for LS wind generator drives but usually at very low-power levels, Hao et al: 2012 [73], Lin et al: 2011 [74], Dobzhanskyi et al: 2012 [75], Yan et al: 2009 [76], Kim, Li and Sarlioglu: 2017 [77] and Xu et al: 2015 [78]. Although attempts have also been made in HS traction drives ${ }^{11}$ as documented in Kim, Li and Sarlioglu (2017) [77] and Xu et al (2015) [78], it is more commonplace to have such designs operated in LS drivetrains which guarantee high average torque values.

Moreover, advancements in SSC technology is one other reason why such LS designs are mostly favoured, Polinder, et al: 2013 [7]. However, AFMs and TFMs are known for their difficult analyses and manufacturing process, which could be scaled-up for multi-MW designs.

With regards to HS wind generator drives, not so much is on record in terms of designs based on FSMs. In Yu and Niu (2015) [79], a new single-phase magnetless FSM was developed for rooftop WPG with rated power of less than $1 \mathrm{~kW}$. Also, the study by Thomas, Zhu and Jewell (2011) [80] demonstrated the feasibility of PM-FSMs for a high-speed and high power $(50 \mathrm{~kW})$ generator applications. Besides issues regarding low gearbox reliability, core loss in PM-FSMs designed for HS wind generator drives can be very high due to a high fundamental frequency, which is generically double in FSMs compared to conventional machines.

The study by Ojeda et al (2012) [37], which considered the RFM structure of a ferrite PMFSM, is a departure from the typical AFM and TFM structures, as well as in the use of rare-earth PMs, popular for such wind generator applications. However, their machine was designed for smallscale LS wind turbine applications. But a cost comparison performed in the study show that the use of ferrite PM is a cheaper option compared to rare-earth PM.

Similarly, Wang et al (2013) [81] proposed a RFM structured WF-FSM with high power density for LS wind energy system. However, because the machine is based on high-temperature superconductor (HTS) field windings, manufacturing cost is bound to be very high with such incipient winding concept.

To the best of the researcher's ability, studies that are committed to the design of FSM for

\footnotetext{
${ }^{11}$ The researcher is not surprised by the domineering presence of PM-FSMs in HS traction applications, whether structured as RFMs, AFMs or TFMs. This is due to the fact that PM-FSM possess good flux weakening capabilities, Pang et al: 2007 [47], Amara et al: 2005 [54] and Fasolo, Alberti and Bianchi: 2014 [69]. Also, as earlier mentioned, the fear of PM demagnetization during flux weakening operation in PM-FSMs is minimal compared to most electrical machines - a characteristic which is mostly desired in such applications.
} 
geared MS wind generator drives based on a simple RFM structure is not easily researchable, until 2014 when the idea struck. As earlier appraised in section 1.2 for conventional geared MS wind generator drivetrains, the size of the gearbox compared to a HS system, as well as that of the generator compared to a LS system, are not only smaller in both cases, but lead to better overall CoE versus performance. Perhaps, the same outcome might be applicable to FSM wind generator drives.

On the other hand, given the low PM utilization ratio notorious with high-energy PM materials such as rare-earth PMs in the design of FSMs, coupled with a very high market monopoly of these rare-earth PMs which make their costs very unpredictable, and the need to completely replace them with either ferrites or wound-fields ${ }^{12}$ cannot be overemphasized.

Now, concerning the use of a simple RFM FSM structure, it should be said that there are many variants such as the E-core, $\mathrm{C}$-core and multi-tooth topologies illustrated in Fig. 1.9, compared to the U-core topology with all poles wound and alternate poles wound as shown in Fig. 1.10. It is expedient to note that all the different topologies come with their advantages and disadvantages, but the most prominent and widely researched of them all is the three-phase 12-stator slots/10-rotor poles (12/10) all poles wound design shown in Fig. 1.10(a), and first conceived by Hoang, Ben Ahmed and Lucidarme (1997) [58]. The 12/10 design possesses important qualities such as symmetrical backEMF waveforms, high torque density and absence of unbalanced magnetic pull (UMP). Its closest competitor is the 12/14 machine, also initially proposed by Chen et al (2009) [82].

Similarly, the WF-FSM was first patterned to mimic the PM-FSM structure depicted in Fig. 1.10(a) by Tang et al (2012) [67], whereby they showed that, given the same phase electrical loading, the 12/14 WF-FSM is able to produce torque at comparable levels to its PM-FSM counterpart. Their finding is unlike what can be achieved in other existing RFM WF-FSM structures e.g., the WF-FSM structures proposed in Zhou and Zhu (2014) [70] and Zulu, Mecrow and Armstrong (2010) [83], which promote redundancy in the way their field and phase coils are arranged. Besides, the 12/8 topology in Zulu, Mecrow and Armstrong (2010) [83] adopts a segmented-rotor which, unlike 12/10 and 12/14 conventional toothed-rotor schemes, exhibits non-sinusoidal back-EMF qualities with potentially high total harmonic distortion (THD), among other things.

Coming back to the 12/14 design, it is known to yield relatively higher torque and lower torque ripple compared to the 12/10 design, Chen et al (2009) [82] were able to prove this. In appearance, the position of its phase $B$ and $C$ coils compared to the 12/10 machine shown in Fig. 1.10(a) are swapped to facilitate a balanced symmetrical back-EMF waveforms, Chen and Zhu: 2010 [65].

\footnotetext{
${ }^{12}$ Note that, the choice to completely replace the rare-earths instead of forming hybrids with either ferrites or wound-fields is to simplify the manufacturing as much as possible.
} 


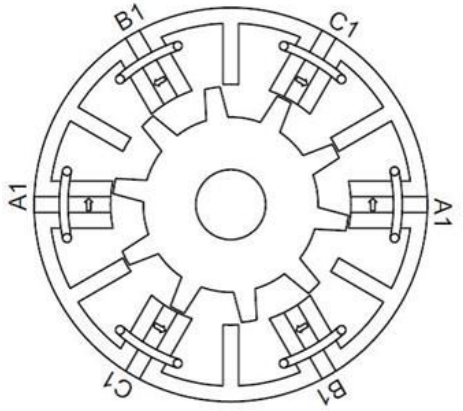

(a)

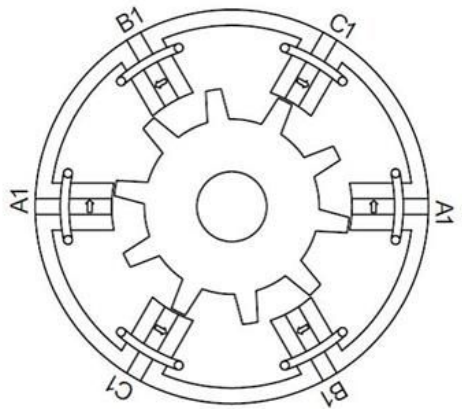

(b)

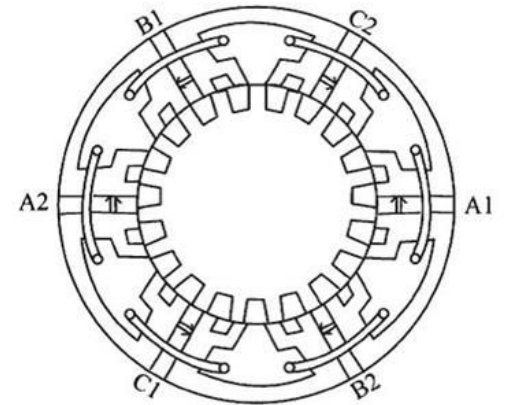

(c)

Fig. 1.9. PM-FSM stator topologies: (a) E-core, (b) C-core, and (b) multi-tooth [44], [64].

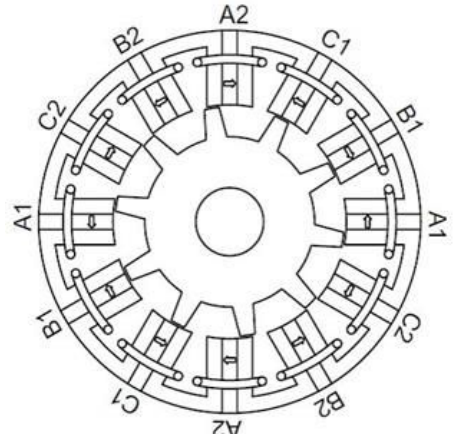

(a)

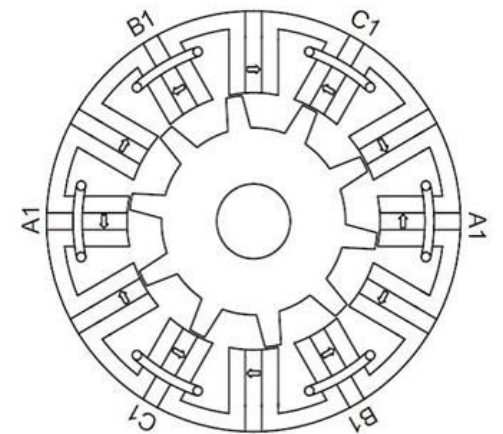

(b)

Fig. 1.10. 12/10 PM-FSM: (a) all poles wound, and (b) alternate poles wound [65].

Interestingly, both the 12/10 and 12/14 have desirable qualities that adequately suit them for the proposed geared MS wind generator drives. For instance, at rotor speed of $360 \mathrm{r} / \mathrm{min}$, the 12/10 and $12 / 14$ both have fundamental frequencies of $60 \mathrm{~Hz}$ and $84 \mathrm{~Hz}$, respectively. Thus, based on a higher fundamental frequency and rotor iron mass, the 12/14 machine might not fully dominate in its efficiency performance due to potentials for higher core losses.

With respect to the use of rare-earth PMs in the design of FSMs, they are very common, even among studies focused on wind generator drives. But as earlier mentioned, not one of these studies have emphasised such designs for geared MS wind generator drives. Furthermore, the advantage of using rare-earth-free materials in FSMs for proposed generator drivetrain cannot be overemphasized. For example, ferrite PMs provide cheaper, low-cost field materials compared to more expensive rare-earth PMs. Notwithstanding, due to low remanence quality, ferrite PMs are prone to high demagnetisation risks. But, as mentioned earlier, the flux focusing abilities as well as the low demagnetisation risks encountered in FSMs generally make them adaptable. Besides, it is also not to be doubted whether the high torque ripple values notorious in FSMs could be abated by the use of ferrite PMs or even wound-fields, since high airgap flux densities in rare-earth-PM-designed PM-FSMs have also been incriminated as one of the causes for high torque ripples, Hua et al: 2008 [46].

As for WF-FSMs, they bring on the table qualities which are not possible with the generality of PM-FSM wind generator topologies. For instance, they can easily achieve constant output armature 
voltage by simply varying the field current under varying wind speed conditions. Also, the maintenance problems associated with slip rings and brushes are non-existent. When designed for MS geared wind generator drives, there is an obvious tradeoff in terms of the core losses and active material costs which they would incur if they were otherwise designed for either HS or LS drivetrains, respectively. To this end, it remains to be seen how the 12/10 or 12/14 FSM could provide a reliable drivetrain solution in geared MS systems compared to HS and LS systems, especially in respect of rare-earth-free designs.

Besides, for the WF-FSM topology proposed by Tang et al (2012) [67], the study was basically on finite element analyses (FEA) on the 12/14 machine, whereby the researcher is yet to encounter experimental validation on this proposed topology. Perhaps, the reason why this has lingered may not be unconnected to the fact that the manufacturing process of this particular topology poses a major challenge in terms of an inherent complication in assembling the field coils unto the stator cores. However, the researcher hopes that, should the same modular nature used for the design of its PMFSM counterpart be adopted, this difficulty may be collapsed.

Also lacking in the printed literature, is a well laid out formulation for the sizing and design of the generality of WF-FSMs as is usually the case for PM-FSMs, e.g., Somesan and Viorel (2013) [68], etc. The researcher is confident that if the design process can be explicitly laid out, it could provide a template for enhancing further research on such WF configurations.

Thus, considering the 12/10 machine, only those designed with rare-earth PMs and ferrite PMs have been studied so far, e.g., Amara et al (2005) [54], etc. No mention has been made on the design and performance study of this topology with regards to a wound-field topology, except in Tang et al (2012) [67] where only a symbolic representation was made. Essentially, the three-phase 12/10 machine, as it is known, has been popularised due to bipolar flux linkage waveform, high torque capability and sinusoidal back-EMF waveform, which should portend similar potentials in wound-field designs. To embark on this, is highly a probable first. In addition, the construction and experimental feasibility of these WF-FSMs is obviously yet to be attempted, neither have there been dedicated studies on their utility-scale designs.

But, concerning large-scale wind turbine installations, particular focus has to be given to the evaluation and performance characteristics in scaling up the generality of these FSM topologies from small-scale kW designs to multi-MW systems. Apparently, such large-scale power investigation is also a new initiative for any of the 12/10 and 12/14 machines. In the literature, only two of such inquiries have been reported to the researcher's best knowledge such as in Wang et al (2013) [81] who proposed a 'low-speed' 'high temperature superconductor' FSM and in Ditmanson et al (2013) [84] 
who proposed and designed a novel $500 \mathrm{~kW}$ 'low-speed' 'modular' PM-FSM drive.

For these reasons, the researcher intends to consecrate the 12/10 and 12/14 FSMs for the proposed geared medium-speed wind generator drivetrains. This is to ensure their performance is evaluated, based on certain optimum design criteria and procedures. The design criteria should include, but are not limited to, minimum mass, maximum efficiency, maximum torque density, minimum torque ripple and maximum power factor, which are considered at prescribed operating power levels. Although it can be argued that so many other RFM structured FSM topologies are also in existent today and are still very much adaptable to the proposed wind generator drives, including single-phase designs, however, not all of these other topologies can boast of the simplicity, maturity, magnetic stability and better torque quality of the 12/10 and 12/14 machines as emphasised in studies by Chen and Zhu (2010) [65], Fasolo, Alberti and Bianchi (2014) [69] and Raminosoa et al (2015) [71].

By way of summary, the tree diagram shown in Fig. 1.11 is produced to highlight the major flow on the current research based on the established state-of-the-art on FSMs for wind generator applications. An artist impression of the proposed wind generator drivetrain is thereafter provided in Fig. 1.12, which would be religiously alluded to in the course of this study progresses. Although, the researcher is motivated by the drivetrain performance of the major components flanked by the wind turbine rotor and the grid, the current study is primarily focused on the optimum design and performance of the wind generator itself in relation to other drive components.

The reason for pursuing the aforementioned design parameters for the proposed geared MS wind generator drive is incumbent on the following important considerations:

- Minimum mass: It is without doubt that the total component mass of the wind generator plays a significant role in determining not only the size, but both the capital and running costs of the general wind turbine assembly. Therefore, minimising the generator mass is bound to immediately lower the drivetrain manufacturing and installation costs.

- Maximum efficiency: Because of the limited maximum power (less than $60 \%$ ) which can be captured by the wind turbine blades from the wind resource, a high efficiency is important to improve the cost-energy ratio of not only the wind generator, but the overall system.

- Maximum torque density and power factor: A high torque density is also beneficial to the size, cost and energy yield of the wind generator, while an improved the power factor is bound to reduce the size and cost of the inverters.

- Minimum torque ripple: A low torque ripple is critical to the survival of the drivetrain since they are sources of mechanical stress. Thus, because FSMs are generally known to suffer from high torque ripple values, the purported target to alleviate them is considered apt. 


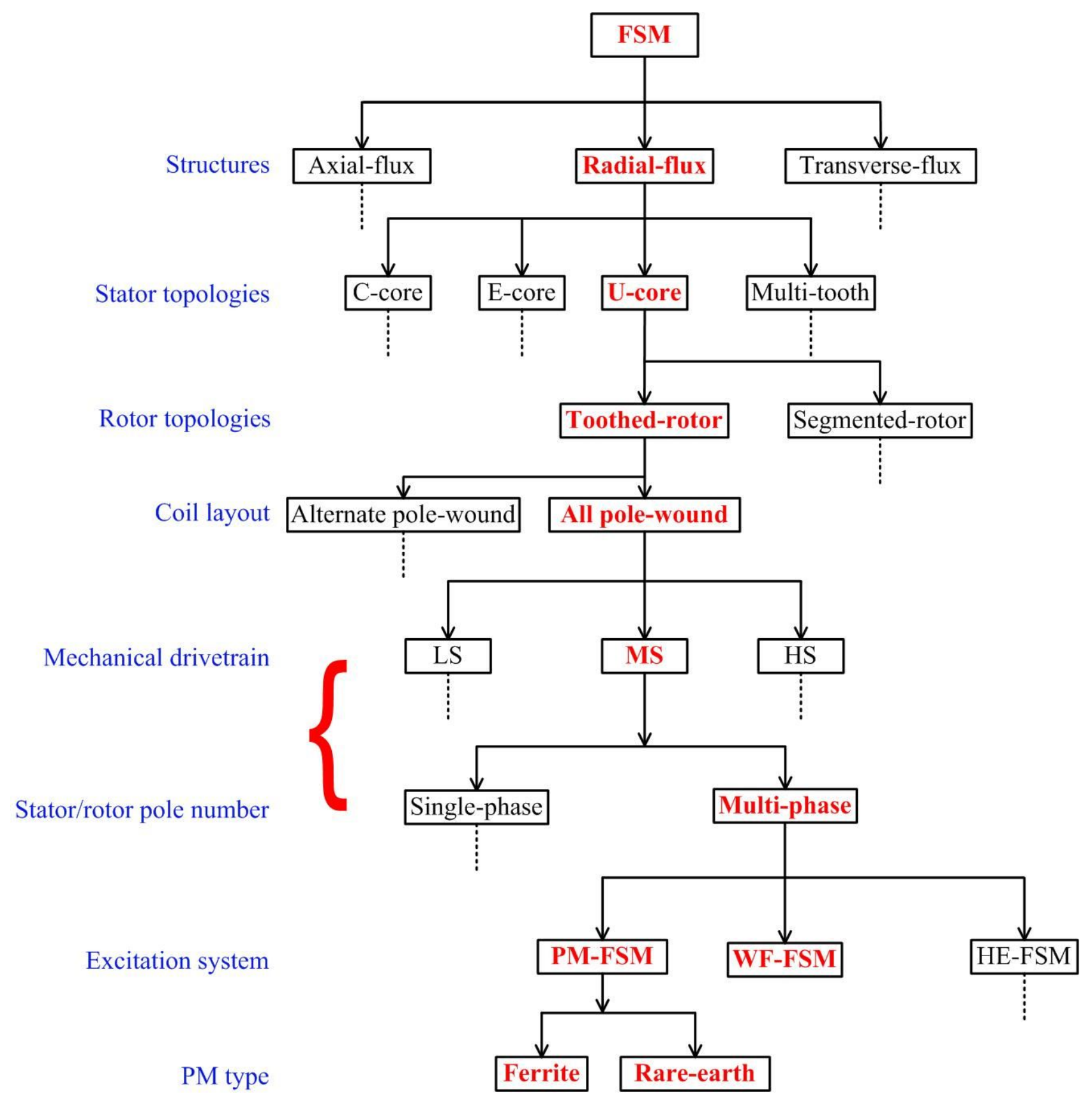

Fig. 1.11. Tree-diagram depicting the state-of-the-art in FSM wind generator drives.

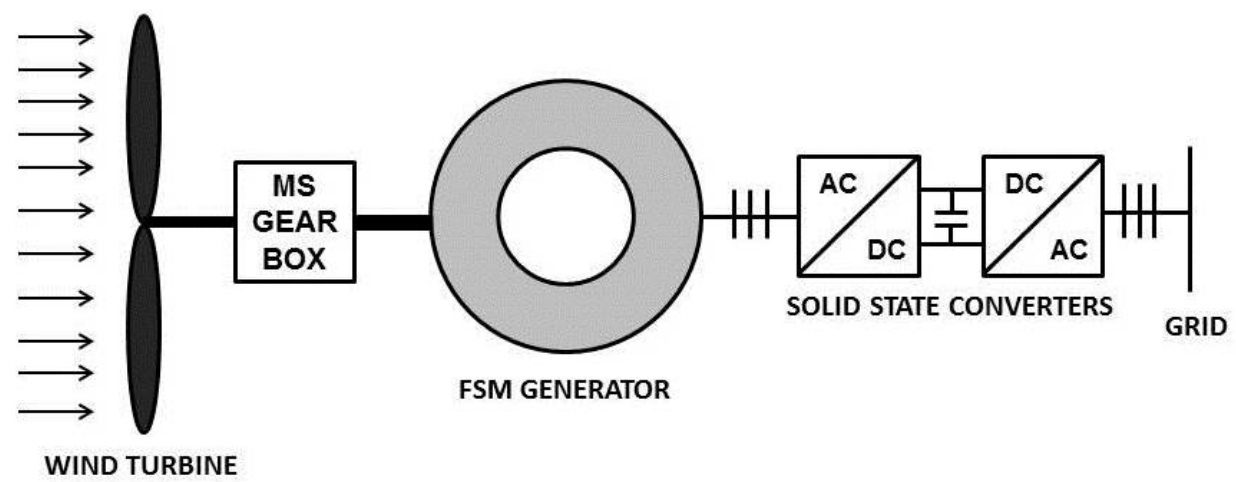

Fig. 1.12. An illustration of the proposed geared medium-speed wind generator drivetrain. 


\subsection{Defining the Problem}

Now that the researcher has established a background for the current dissertation, it is necessary to still provide additional information on what has been documented about the design optimisation cum performance analyses of the 12/10 and/or 12/14 RFM structured FSMs. By so doing, a clearer and narrower direction on the proposed research can be articulated.

Hence, over the years, the design, analyses and performance characteristics of the proposed 12/10 and 12/14 machines have been demonstrated in many studies. A track of some of these studies are summarised as follows:

- Amara et al (2005) [54] presented a design procedure of the 12/10 machine using both ferrite and rare-earth PMs, based on geometry parameterisation (no optimisation) and FEA. The machines were designed at $2 \mathrm{~kW}$ for HS airborne applications, and compared in terms of mass, cost, and temperature sensitivity. The comparison reveals, among other things that, although the rare-earth machine uses fewer magnets, it is much more expensive than the ferrite design.

- Based on a $2 \mathrm{~kW}, 1500 \mathrm{r} / \mathrm{min}, 12 / 10$ machine, Hua et al (2006) [61] proposed a general design procedure for PM-FSMs by replacing the traditional $D_{\text {in }}^{2} l_{s t}$ sizing equation with $D_{\text {out }}^{2} l_{s t}$. Thus, equipped with only the power requirements and stator/rotor topology, among others, they confirmed by FEA and experimental results that the volume of the machine and other dimensional parameters can be so determined.

- Pang et al (2007) [47] compared both analytically and by FEA the electromagnetic performance of the 12/10 PM-FSM to an 18/12 IPM machine for traction drives. By considering different split ratios based on parametric analyses, they found that the former has slightly higher torque and flux weakening capability.

- Also, using parametric analyses coupled with FEA, Zhu et al (2008) [62] investigated the influence of design parameters on the output torque of the 12/10 PM-FSM for motor applications. They showed among other things that, if the machine's stator tooth width, stator magnet thickness and slot opening are kept equal, maximum output torque is produced.

- Chen et al (2009) [82] proposed the 12/14 machine for the first time by comparing it to the 12/10 machine. Again, based on FEA parametric evaluations, they were able to show the superior torque and torque ripple performance of the 12/14 machine.

- Chen and Zhu (2010) [65] developed analytically, the general conditions for balanced symmetrical back-EMF waveforms in PM-FSMs. They found that the best stator to rotor pole number combinations must be very close and that with larger rotor pole number, a relatively 
higher torque is obtained. Then concentrating on the 12/10 and 12/14 machines, they validated their proposal both by FEA and experiment at a rated speed of $400 \mathrm{r} / \mathrm{min}$.

- Xu et al (2011) [50] proposed four rotor-based cogging torque reduction schemes (rotorpole pairing, rotor pole-notching, rotor-pole chamfering, and rotor-pole skewing) to improve the adaptability of the 12/14 machine for HS plug-in hybrid electric vehicles (PHEV). Eventually, six important performance indexes, including the cogging torque, were normalized with respect to the original rotor scheme and compared in FEA. The results showed that the best effects of the four is the rotor-pole pairing scheme.

- Tang et al (2012) [67] proposed, for the first time, the wound-field version of the 12/14 machine for enhanced field weakening capabilities. Their design, which preserves the flux focusing effect and compares shoulder-to-shoulder with its PM counterpart, was fashioned for an electric truck and analysed using FEA.

- Somesan and Viorel (2013) [68] embarked on design optimisation based on deterministic algorithms and performance analyses of a $3000 \mathrm{r} / \mathrm{min}, 30 \mathrm{~kW} \mathrm{12/10} \mathrm{machine} \mathrm{using} \mathrm{analytic} \mathrm{ex-}$ pressions concocted from the design-sizing theory. Later, FEA was adopted to validate the electromagnetic performance of the optimally designed machine, as well as establish the positive influence of rotor-pole shaping on cogging torque reduction.

- McFarland, Jahns and El-Rafaie (2014) [55] provided insights on the static demagnetisation characteristics in PM-FSMs using the 12/10 machine. They found that, due to the arrangement of the PMs and the stator coils, their MMFs (PM and current) seem to form common partnership, thereby ensuring minimal demagnetisation risks in the PMs during normal operation, "regardless of the current's axis orientation or polarity". According to them, this peculiar behavior underscores the prospects of using less expensive rare-earth-free materials.

- Based on the 12/10 and 12/14 machines, Raminosoa et al (2015) [71] assessed the potentials of three different designs, which either reduce or remove rare-earth materials, for HEV traction applications. They exploited the special magnetic configuration of FSMs already broadcasted by McFarland, Jahns and El-Rafaie (2014) [55] to justify two of the designs which used PMs, with the key design requirements being $55 \mathrm{~kW}$ peak power at $2800 \mathrm{r} / \mathrm{min}$ base speed. The third one was designed using only wound-fields, whose topology is different from that proposed in Tang et al (2012) [67]; hence, it could not satisfy the efficiency requirement among the three designs. Among other things, they found as usual that, the 12/14 machine has a higher torque density than a 12/10 machine, with higher efficiency in the latter due to reduced losses.

Based on the prevailing literature on the design, analyses and performance of the proposed 
$12 / 10$ and 12/14 machines, it is commonly observed that a well thought out design optimisation that accommodates multiple design objectives, sequel to some constraint functions, is fundamentally absent. A good number of the published works have been so dedicated to parametric resolution for the optimal design (Ojeda et al (2012) [37] for example), and most times they confuse such "sensitivity analyses" for "design optimisation". Where this is not the case, deterministic algorithms are invoked to optimise these machines based on single objective problems or their hybrids, such that they fail to promote the absolute optimum of such design ventures.

Moreover, an existing framework which is established on the widely utilised design-sizing method as proposed in Hua et al (2006) [61], used in Somesan and Viorel (2013) [68], and emphasised in Zhang et al (2009) [45] to trigger this process, is still not fully exploited. When this technique is fully deployed, an initial design that can be conceptualised to activate the optimisation process is produced. Thereafter, it becomes easy to collectively evaluate a part or all of the aforementioned design requirements such as power factor, torque ripple and torque density among others, bearing in mind, the optimum performance of the associated drivetrain components.

Granted, the study by Somesan and Viorel (2013) [68] does not address a single objective problem per se since the objective function considered is to maximise the torque density, nevertheless the approach falls short because it was a non-constrained deterministic (i.e., gradient-based) search method, with only five design parameters considered. This point is stressed because in the optimal design of electrical machines, a good number, both of the design parameters and the design requirements, which hustle for relevance, must be taken into account initially, Duan and Ionel: 2013 [87] and Stipetic, Miebach and Zarko: 2015 [88]. Thus, if the competition is not properly arbitrated by the designer, the resulting optimal solution is merely a local optimum. For this not to happen, the preferred optimisation methods should be multi-objective stochastic (non-gradient) based algorithms. As quipped in Vanderplaats (2007) [99], it does go without saying that the researcher understands fully, that no given optimisation problem, especially with so-called non-convex problems, can guarantee a finite optimum solution without a quid pro quo. But when the case for realising a design, as close as possible to the global optimum is admitted, the satisfaction of the imposed design criteria comes without doubt.

Short of reference to any application area, it still must be said that other studies have optimised and/or compared the 12/10 and 12/14 machines. For instance in terms of rare-earth-free designs, studies by Zhou and Zhu (2014) [70] and Sulaiman, Kosaka and Matsui (2012) [95] only dealt on deterministic optimisation methods in their studies; whereas Raminosoa et al (2015) [71] and Tang et al (2012) [67] processed some design and comparison aspects, but without any form of optimisation 
mentioned. Thus, to the best of the researcher's knowledge, it appears that for the relevant design requirements, they are yet to be collectively optimised for best results of the 12/10 and 12/14 machines, especially in line with the proposed geared MS wind generator operation.

An example of a multi-objective and multi-constraint optimisation problem is reported for IPM synchronous motor for HEV/EV applications in Zhang, Ionel and Demerdash (2016) [89], wherein "three concurrent objective functions are minimized: material cost, losses, in order to ensure high efficiency, and the difference between the rated and the characteristic current, aiming to achieve very high constant-power flux-weakening range" [sic.], having a minimum of ten design variables and three constraints for a prescribed problem. As expected, their study highlighted the use of stochastic algorithms e.g., differential evolution, which can produce a population of non-dominant optimal solution as a Pareto optimal set. Remarkably, a search by the researcher reveals that the Pareto effect has never been attempted for any combination of performance parameters in the design optimisation of the proposed $12 / 10$ and $12 / 14$ machines.

In the meantime, it has to be said that stochastic algorithms do not come without their concerns in the design optimisation of electrical machines. The main challenge posed is that they are computationally expensive and time consuming to implement. Furthermore, the use of FEA-based numerical optimisation, in tandem, is considered an additional burden to the computation time, viz., costs. But, FEA is, without doubt, both robust and highly efficient for electrical machine design and analyses. To do otherwise, would suggest that increased accuracy is being sacrificed on the altar of enhanced speed in the process chain. To the researcher, the former situation is preferred to the latter for the present inquiry. Notwithstanding, the fear of aggregating both the computer memory and design cycle may not be deep seated, as the researcher intends to adopt a "powerful and efficient" twodimensional (2-D) static FEA optimisation program (SEMFEM) developed by Gerber (2011) [91], with script-based interface and a propensity for parallel processing which speeds up the process in any case.

However, SEMFEM which is based on 2-D FEA modelling is limited by the fact that compared to 3-D FEA solutions, the end-winding effects can result in huge disparities for the proposed FSMs. Even more, seeing that the case being made for rare-earth-free designs includes the presence and arrangement of the phase coils over the field coils in the WF-FSM topology, usually with a very large split ratio in comparison to the aspect ratio, it becomes indispensable to devise an approximate calculation which accounts for the end-winding effects in 2-D FEA. Besides, to devise such endwinding approximation is also for the sake of the design optimisation procedure converging to the true optimal design. 
Hence, the method of considering only the end-winding resistance and ignoring the endwinding inductance as done in Zhou and Zhu (2014) [70] is limited for design optimisation, same as for prescribing a fixed margin to account for end effects as done in Raminosoa et al (2015) [71]. To this end, the incorporation of an approximate formula which captures, in real-time, the end-winding effect in the 2-D FEA is meant to enhance the accuracy of the design optimisation process.

Based on the foregoing, the researcher, at this juncture, formally proposes the use of FEA solutions interfaced with a robust multi-objective stochastic algorithm in the design optimisation and performance evaluation of the 12/10 and 12/14 FSMs benchmarked for geared MS wind generator drivetrains. In pursuit of the underlying research objectives, additional emphasis is to be placed on the generators' design characteristics for both small-scale and utility-scale systems.

\subsection{Methodology}

In the proposed study, FEA is to be copiously engaged. To this end, the design optimisation anticipated is heavily based on 2-D static FEA solution of a number of performance quantities arising from varying the feasible stack volume on the one hand, as well as other relevant constrained dimensional (in the radial geometry) and non-dimensional design variables of the proposed machines. However, before then, the sizing of each machine would be incurred using the sizing-design theory popularised by Huang et al (1998) [92], but further embellished to suit the current study. Because the sizing-design technique is to be frequently invoked in the current study, the whole procedure in relation to FSMs has been annotated in Appendix A1.2.

After undertaking the conceptual sizing, constrained multi-objective, multi-variable FEA design optimisation would be administered on the initial designs. In order not to increase the time for the FEA-based optimisation, as earlier quipped, so-called SEMFEM, an in-house time-stepping 2D static FEA package, devised by Gerber (2011) [91], would be applied. Compared to commercial FEA packages, SEMFEM has potentials to speed up the simulation time, without compromising the design accuracy. The speed enhancements in SEMFEM is mostly facilitated by its script-based nature adopted in solving most of the machine performance parameters, which is simply facilitated by first obtaining the phase flux linkages and transforming them into dq quantities for each static step covered within a time period. The complete details on the functionalities of SEMFEM and its FEA capabilities are fully captured in the dissertation report by Gerber (2011) [91].

To this end, SEMFEM comes with an added library which enables it to perform the optimisation of electrical machines, by simply coupling it to an external optimiser. The optimiser preferred for this task is the commercial VisualDOC suite, VisualDOC: 2017 [93]. In respect of this, the flowchart 
in Fig. 1.13, which is very fundamental to the design optimisation methodology assumed throughout this thesis, is devised to simplify the execution of the design optimisation workflow.

As earlier discussed in the preceding section, the importance of end-winding effects cannot be overruled in the design optimisation of electrical machines. Without 3-D FEA modelling, the accuracy of mimicking the end-winding characteristics in 2-D FEA predictions is usually very limited. However, the incorporation of an approximate expression which evaluates such effects in real-time could be very vital in a design optimisation process. Considering that the proposed 12/10 and 12/14 FSM topologies are typically non-overlap winding machines, thus, an approximate formula as proposed for similar non-overlap PM winding machines in Potgieter and Kamper (2014) [94] is to be adapted for this purpose, in respect of both the PM and wound-field designs.

Also, it is appropriate for any outcome of the design optimisation process, at each stage of the inquiry, to be lightly validated. Mostly, such validations would be undertaken in 3-D transient FEA simulations, using a commercial FEA package called ANSYS Maxwell ${ }^{\odot}$. This process will continue until at last, a $10 \mathrm{~kW}$ WF-FSM optimal design candidate is prototyped and experimentally tested.

\subsection{Objectives}

In the beginning of this chapter, the importance of wind generators in the wind energy drivetrain was set forth. Subsequently, an assessment of the different wind generator drivetrains which are in existence and their value chain in terms of energy yield per cost was provided. Obviously, among the three established drivetrains, the geared MS wind generator drives stands tall as the best drivetrain solution in sync with traditional wind generators like DFIGs and PMSGs. With some known limitations encountered in such traditional wind generator concepts, especially when designed at utilityscale power levels, the need to begin to consider the use of non-conventional wind generator concepts, viz., so-called stator-active (brushless) machines, in the geared MS drivetrain, becomes pertinent.

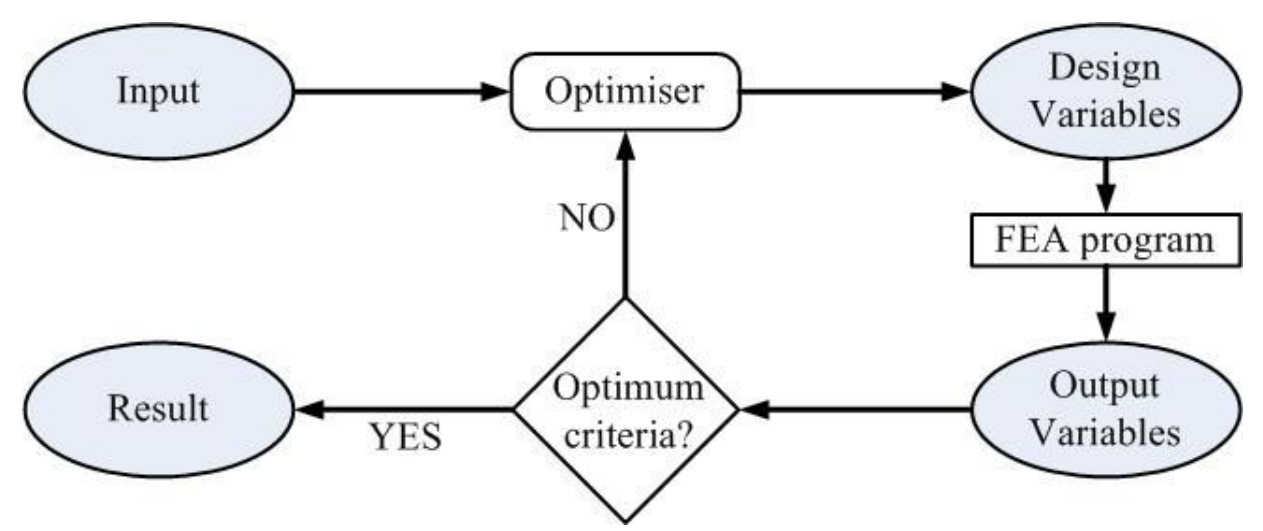

Fig. 1.13. Outline of the proposed 2-D FEA design optimisation process. 
One of such stator-mounted machines, the FSM, is gaining increased attention because of key features such as high power density, high efficiency, good thermal management and a robust rotor structure compared to conventional machines, which makes it a suitable candidate for the proposed wind generator drivetrain. To this end, the candidature of FSMs for geared MS wind generator drivetrains is being proposed in this thesis, for the first time, in its very simple and very popular generator topologies. To achieve this aim, the following specific objectives are to guide the study:

- Evaluate the different drivetrain performance of the nominated FSM wind generators.

- Formulate the procedure to perform multi-objective design optimisation (MDO) of the proposed FSMs in geared MS drivetrain.

- Investigate the use of rare-earth-free excitation for the proposed FSM wind generator drives.

- Compare the performance of the proposed FSM wind generator drivetrains for small-scale kW designs to utility-scale MW designs.

- Manufacture and experimentally test a $10 \mathrm{~kW}$ prototype based on the proposed FSM wind generator drivetrain.

\subsection{Thesis Layout}

The remainder of this dissertation is structured according to the following chapter topics and in pursuit of the stated research objectives:

- Chapter 2 is used to provide insights on the comparison of the different drivetrain solutions of optimally designed 12/10 rare-earth PM-FSMs in terms of CoE versus performance. In addition, it gradually presents the steady-state FEA design and modelling adopted throughout the study.

- In Chapter 3, the design optimisation and comparison of the 12/10 and 12/14 rare-earth PMFSM at $10 \mathrm{~kW}$ and $3 \mathrm{MW}$ power levels is presented for the proposed geared MS wind generator drivetrain. The main purpose of this chapter is to monitor how by scaling, the performance of both machines, based on some key design parameters, is affected.

- In Chapter 4, the possibility of using rare-earth-free field excitation sources, viz., ferrite PM and wound-field, in a 12/10 flux switching machine designed for wind energy drives is gradually initiated for small-scale power. The study involves the performance comparison of these so-called low-cost FSM variants for geared MS wind generator drives on one hand, and the investigation of the demagnetisation effects of the ferrite PM-FSM in lieu of its rare-earth PM-FSM counterpart. The aim is to limit dependence on the use of rare-earth PMs.

- Chapter 5 presents the formulation and multi-objective design optimisation (MDO) of WF- 
FSMs. The major highlight here is on the different approaches to the MDO problem and the influence of the respective outcomes on the generator drivetrain performance.

- Chapter 6 details the design characteristics of rare-earth-free FSMs by comparing their performance in small- and large-scale power levels. To evaluate their potentials, a comparison is further made with the $3 \mathrm{MW}$ rare-earth PM-FSMs earlier established in Chapter 3 and other conventional geared MS wind generators designed at utility-scale wind turbines.

- Chapter 7 briefly discusses the processes of manufacturing and experimental testing of a 10 $\mathrm{kW}$ WF-FSM prototype developed in the laboratory.

- Chapter 8 is documents the general conclusion, unique contributions and future recommended areas of research based on the broad-spectrum of wind energy research and development, especially as it relates to the specific studies escalated in this dissertation. 


\section{ChAPTER Two}

\section{Drivetrain Performance and Comparison of PM- FSMS}

This chapter is written to highlight the competitive performance of PM-FSMs in different wind generator drivetrains. Only one of the proposed generator topology is considered here i.e., the 12/10 machine. At first, some background information is provided. Then, the design process is presented, detailing the basic performance variables of the PM-FSM in steady-state dq expressions, as well as prescribing the optimisation problems. Thereafter, important features of the different wind generator drivetrains are evaluated and compared, especially in terms of $\mathrm{CoE}$ versus performance. Note that, some of the ideas portrayed in this chapter have been previously reported by the researcher in a conference paper as Akuru and Kamper (2015) [98], and later accepted in a journal volume, Akuru and Kamper: 2015 [97].

\subsection{Introduction}

In recent times, wind turbine manufacturers have increasingly shifted their focus to geared MS drivetrains for wind turbine systems because of some challenges encountered in LS and HS systems, such as higher manufacturing and maintenance costs, de Vries: 2012 [13]. Also, there is an increasing drive to tap the vast wind energy potential which exists both onshore and offshore, with a guaranteed good return on investment (ROI) when wind turbines are designed at industrial-scale power levels. Hence, geared MS systems present a compromise for such industrial-scale wind turbines, in terms of yielding a good tradeoff in both the gearbox and generator sizes. Recent trends which show the benefits of geared MS drivetrains over LS and HS drivetrains have been already addressed in section 1.2 in Chapter 1, particularly in Figs. 1.3 and 1.4 mined from Schmidt and Vath (2012) [20], which dealt on the drivetrain performance comparison of PMSGs.

Also, studies by Cao, Xie and Tan (2012) [4] on geared MS DFIGs and Bang et al (2008) [29] on geared MS PMSGs both portrayed lowest CoE relatively compared to LS and HS systems. Consequently, to capitalise on the stated benefits, there is need to do further research to highlight important qualities of newer concept of wind generators designed for geared MS drivetrains, while targeting highest CoE. Such studies appear not to have been documented for FSMs - typically, a nonconventional machine - gaining wide usage because of its stator-mounted (and brushless) qualities, Rauch and Johnson: 1955 [40] and Cheng et al: 2011 [44]. 
In this chapter, an attempt is made to design, optimise and compare performance of the very popular 12/10 radial-flux PM-FSM topology in the different wind generator drivetrains, for the first time. To achieve this, 2-D static FEA method is employed for both the optimisation and the performance evaluation. Eventually, the design feasibility is established by 3-D FEA process applied to a benchmark design.

\subsection{PM-FSM Electromagnetic Modelling}

Due to the fact that the design optimisation and performance evaluation is undertaken by means of an in-house Python script-based non-linear 2-D static FEA program, so-called SEMFEM developed by Gerber (2011) [91], it is important to set forth, for the FSM, the steady-state d- and q-axes (dq) equations as implemented in situ. Besides, these dq expressions are an additional means to analytically evaluate relevant performance parameters when called upon in the course of this dissertation. This way, the speed of the design optimisation process is hopefully enhanced.

Consequently, the steady-state dq equivalent circuits and phase diagram, in generator mode, are shown in Fig. 2.1, with $\Delta$ and $\alpha$ representing the load and current angles, respectively. Note that, $V_{s}>E_{q}$ and that $I_{s}$ lags $V_{s}$ as implied in Fig. 2.1. Based on the evaluation of the 2-D FEA model, the $\mathrm{dq}$ axes flux linkages, from which other machine characteristics are devised, are given as

$$
\begin{aligned}
& \lambda_{d}=\lambda_{M}+L_{d} I_{d}, \\
& \lambda_{q}=L_{q} I_{q},
\end{aligned}
$$

where $\lambda_{d}$ and $\lambda_{q}$ are the transformed dq flux linkages, $I_{d}$ and $I_{q}$ are the dq phase currents, $L_{d}$ and $L_{q}$ are the dq axes inductances, and $\lambda_{M}$ is the no-load flux linkage.

The expressions in (2.1) and (2.2) are meant to facilitate the determination of the dq inductances. In reality, the dq flux linkages are fundamental output variables resulting from the SEMFEM technique which prescribes as input variables, the field excitation source and load profile of a proposed electrical machine design. To this end, the 2-D FEA program evaluates the dq quantities mainly from their fundamental phase quantities by using Park's dq transformation equation expressed as

$$
\left[\begin{array}{l}
\Pi_{d} \\
\Pi_{q} \\
\Pi_{0}
\end{array}\right]=\frac{2}{3}\left[\begin{array}{ccc}
\cos \theta & \cos \left(\theta+\frac{2 \pi}{3}\right) & \cos \left(\theta-\frac{2 \pi}{3}\right) \\
\sin \theta & \sin \left(\theta+\frac{2 \pi}{3}\right) & \sin \left(\theta-\frac{2 \pi}{3}\right) \\
\frac{1}{2} & \frac{1}{2} & \frac{1}{2}
\end{array}\right]\left[\begin{array}{l}
\Pi_{a} \\
\Pi_{b} \\
\Pi_{c}
\end{array}\right]
$$

where $\Pi$ can represent the flux linkages, currents or voltages and $\theta$ is the transformation angle of the magnetic field axis (d-axis) of the rotor with respect to the magnetic phase $A$ vector of the stator windings (see Fig. 2.2), measured in electrical radians. 


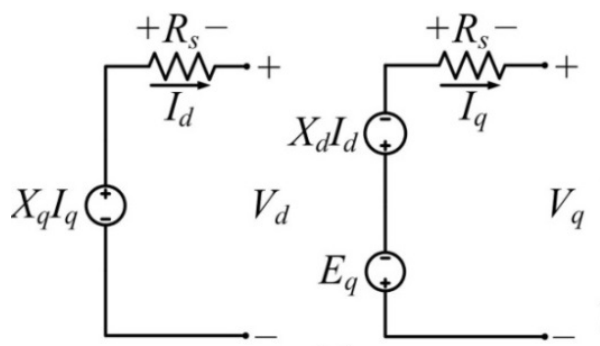

(a)

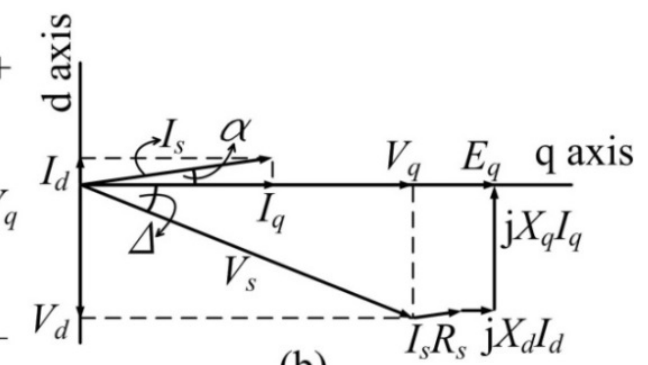

(b)

Fig. 2.1. PM-FSM modelling: (a) dq equivalent circuits, (b) phasor diagram.

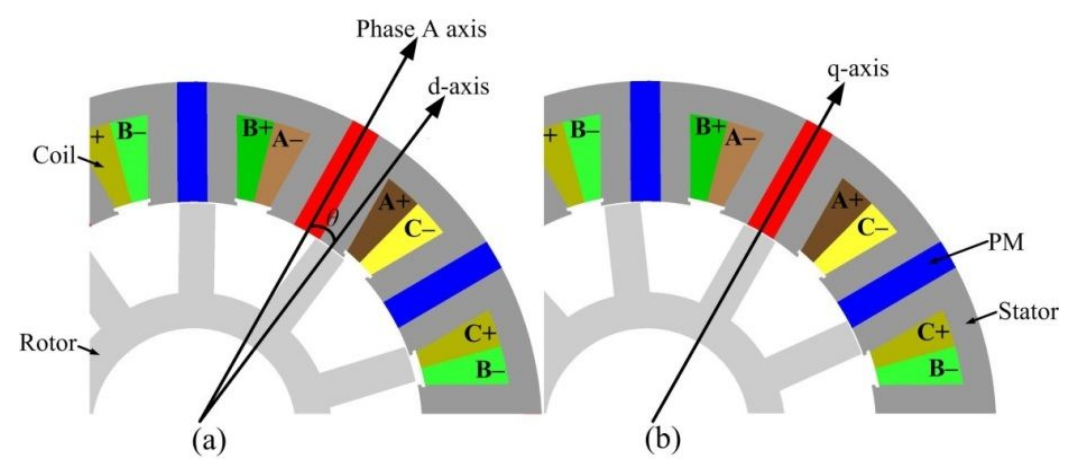

Fig. 2.2. Cut-out illustration of dq-axes rotor positions for 12/10 PM-FSM: (a) d-axis, and (b) qaxis.

The dq axes voltages are evaluated as

$$
\begin{aligned}
& V_{d}=\omega_{e} L_{q} I_{q}-R_{s} I_{d}, \\
& V_{q}=\omega_{e} \lambda_{M}-\omega_{e} L_{d} I_{d}-R_{s} I_{q},
\end{aligned}
$$

where $R_{s}$ is the phase resistance and $\omega_{e}$ is the electrical speed in $\mathrm{rad} / \mathrm{s}$.

The phase resistance is given as

$$
R_{s}=2 q N_{p h}^{2} \rho_{C u} \frac{l_{s t}+l_{e}}{A_{p h}},
$$

where, $q$ is the number of phase coils in series connection, $N_{p h}$ is the turns number per coil for the phase windings, $\rho_{C u}$ is the resistivity of copper wire at room temperature, $l_{s t}$ is the axial length of the lamination core, $l_{e}$ is the end-winding length on one side of the phase coil (see later in subsection 5.3.2 in Chapter 5 for full details) and $A_{p h}$ is the area of the phase wire.

The magnitudes of the phase output voltage and current are calculated as

$$
V_{s}=\sqrt{V_{d}^{2}+V_{q}^{2}}, \quad I_{s}=\sqrt{I_{d}^{2}+I_{q}^{2}}
$$

The electromagnetic torque and torque ripple are given as

$$
\begin{aligned}
\tau_{e} & =\frac{3}{2} N_{r}\left(I_{q} \lambda_{M}+\left(L_{d}-L_{q}\right) I_{d} I_{q}\right), \\
\kappa_{\delta} & =\frac{\tau_{e(\max )}-\tau_{e(\min )}}{\tau_{e}}
\end{aligned}
$$

where $N_{r}$ is the number of rotor poles, and $\tau_{e(\max )}$ and $\tau_{e(\min )}$ are the maximum and minimum peaks of 
$\tau_{e}$ when the machine is operating on-load.

The real and reactive power, total copper and core losses are given as follows:

$$
\begin{aligned}
& \mathrm{P}_{\mathrm{out}}=\frac{3}{2}\left(V_{d} I_{d}+V_{q} I_{q}\right), \\
& \mathrm{Q}_{\mathrm{out}}=\frac{3}{2}\left(V_{q} I_{d}-V_{d} I_{q}\right), \\
& P_{C u}=\frac{3}{2}\left(I_{d}^{2}+I_{q}^{2}\right) R_{s}, \\
& P_{\text {Core }}=C_{m} f_{e}^{\beta} \sum_{k=1}^{N} \dot{B}_{k}^{\sigma} M_{k},
\end{aligned}
$$

where $C_{m}, \sigma$ and $\beta$ are the purported Steinmetz coefficients determined by experiments at different operating frequencies for the laminated iron material. In this thesis, non-oriented fully processed M400-50A gauge magnetic steel grade with mass density $7600 \mathrm{~kg} / \mathrm{m}^{3}$ is the preferred core sheets from which the Steinmetz coefficients are determined, based the core loss versus frequencies curves, yielding $C_{m}=0.003204, \sigma=1.577$ and $\beta=1.924$. $\dot{B}_{k}$ is the peak flux density located in a corresponding iron lamination, $M_{k}$ is the mass of the corresponding iron lamination stack, and $N$ is the total number of regions (usually of teeth and yokes) considered in the iron lamination. In addition, $f_{e}$ is the fundamental frequency of the PM-FSM generator calculated as

$$
f_{e}=\frac{n_{s} N_{r}}{60}
$$

where $n_{s}$ is the rotor shaft speed in $\mathrm{r} / \mathrm{min}$ which typifies the drivetrain operation.

Lastly, the efficiency and power factor are calculated as

$$
\begin{aligned}
& \eta=\frac{\mathrm{P}_{\text {out }}}{\mathrm{P}_{\text {out }}+P_{C u}+P_{\text {Core }}} \\
& \mathrm{PF}=\cos \left(\tan ^{-1}\left(\frac{I_{d}}{I_{q}}\right)+\tan ^{-1}\left(\frac{V_{d}}{V_{q}}\right)\right) .
\end{aligned}
$$

\subsection{Design Optimisation}

Although wind generator drivetrain is implied in this study, the focus of the current design is on the wind generator component itself, whereby conclusions on other associated drivetrain components are drawn. In respect of this, the inquiry is mainly on the performance of 12/10 PM-FSM generator operated under different drivetrains. Thus, the $\mathrm{CoE}$ is of primary interest. The objective functions to be optimised are the ratio of the average torque to PM mass, and the active mass given as

$$
\begin{aligned}
& F_{1}(\bar{x})=\frac{\tau_{e}}{M_{P M}}, \\
& F_{2}(\bar{x})=M_{A}=M_{F e S}+M_{F e R}+M_{P M}+M_{C u},
\end{aligned}
$$

where $M_{P M}$ is the mass of the PM, $M_{F e S}$ is the mass of the stator iron, $M_{F e R}$ is the mass of the rotor iron, and $M_{C u}$ is the copper mass. 
Table 2.1. Boundary conditions defined for design parameters

\begin{tabular}{c|c|c|c|c|c|c|c}
\hline \multirow{2}{*}{ Design variables } & \multirow{2}{*}{$\overline{\boldsymbol{x}}$} & \multicolumn{6}{|c}{ Drivetrain } \\
\cline { 3 - 8 } & & $\boldsymbol{x}^{(\boldsymbol{L})}$ & $\boldsymbol{x}^{(\boldsymbol{U})}$ & $\boldsymbol{x}^{(\boldsymbol{L})}$ & $\boldsymbol{x}^{(\boldsymbol{U})}$ & $\boldsymbol{x}^{(\boldsymbol{L})}$ & $\boldsymbol{x}^{(\boldsymbol{U})}$ \\
\hline Current angle (deg.) & $\alpha$ & 0 & 90 & 0 & 90 & 0 & 90 \\
Current density (A/mm $\left.{ }^{2}\right)$ & $\mathrm{J}$ & 1 & 5 & 1 & 5 & 1 & 5 \\
Stator outer diameter (mm) & $D_{\text {out }}$ & 600 & 700 & 250 & 300 & 170 & 200 \\
Stator inner diameter (mm) & $D_{\text {in }}$ & 388.5 & 500 & 162 & 180 & 120 & 140 \\
Shaft diameter (mm) & $D_{s h}$ & 225 & 300 & 80 & 85 & 50 & 60 \\
Stack length (mm) & $l_{s t}$ & 250 & 500 & 90 & 180 & 70 & 140 \\
PM length (mm) & $b_{p m}$ & 10 & 20 & 5 & 10 & 5 & 10 \\
Rotor pole width (mm) & $b_{p r}$ & 20 & 40 & 10 & 20 & 7.5 & 15 \\
Slot opening width (mm) & $b_{s l s}$ & 12.5 & 25 & 7.5 & 15 & 5 & 10 \\
Stator yoke height (mm) & $h_{y s}$ & 12.5 & 25 & 7.5 & 15 & 5 & 10 \\
Rotor yoke height (mm) & $h_{y r}$ & 10 & 20 & 7.5 & 15 & 7.5 & 10 \\
Rotor tooth tapering factor & $t_{0}$ & 0.5 & 1 & 0.5 & 1 & 0.5 & 1 \\
\hline
\end{tabular}

The design optimisation process is further subjected to the following constraint functions defined as $\mathrm{P}_{\text {out }} \geq 10 \mathrm{~kW}, \mathrm{PF} \geq 0.8, \kappa_{\delta} \leq 10 \%{ }^{13}$ and $\eta \geq 90 \%$. To account for the different drivetrains, the mechanical speed of the generator is constrained at $30 \mathrm{r} / \mathrm{min}, 360 \mathrm{r} / \mathrm{min}$ and 1500 r/min which represent the LS, MS and HS drivetrains, respectively, while the airgap length $(g)$ was kept constant at $0.7 \mathrm{~mm}$ for the three designs. The use of the same airgap length for the three designs may be considered unfair, especially for the LS design, but as would be shown later in Chapters 4,6 and 7, this same airgap length is used for similar sized WF-FSMs. As a matter of fact, Pyrhönen, Jokinen and Hrabovcova (2008) [96] reported that for machines with exceptionally large stator outer diameter, e.g., a LS design, an airgap ratio defined as $g / D_{\text {out }} \approx 0.001$ is preferred. They further stated that for LS designs, a small airgap is necessary to cut down on the amount of PM usage. Hence, the uniform airgap size adopted for the three designs appears to mostly favour the LS design.

The initial design is created according to the sizing-design method presented in Appendix A1.2. Each drivetrain speed is used generate a reference design to initiate the design optimisation in respect of the design geometry. Reasonable boundary conditions are formulated for the twelve design parameters chosen as itemised in Table 2.1, with $x^{(L)}$ and $x^{(U)}$ representing the lower and upper

\footnotetext{
13 The imposition of $10 \%$ constraint on the torque ripple may be considered as very high, especially for the proposed wind generator drives, but as it has earlier been reported in this dissertation, that torque pulsation in FSMs is generally high due to their double salient nature. For instance, the study by Raminosoa et al (2015) [71] which considered two reduced rare-earth PM and one wound-field designs, based on similar design constraints being pursued in this study, obtained torque ripple values at $4 \%, 9 \%$ and $7 \%$, irrespectively. For LS applications, Pyrhönen, Jokinen and Hrabovcova (2008) [96] suggested that torque ripple can be restricted to 5-10\% or less for typical double salient machines. Note that, already, the insights gathered by the researcher from the study by Zhang, Ionel and Demerdash (2016) [89] on the interactions between some major design performance variables, also considered in this study, such as mass, efficiency and power factor in conventional PM machines, is a precursor to defray the torque ripple at $10 \%$, in order to ease the burden in the optimisation search space whenever it is used as a design constraint. Besides, in an attempt to reduce the torque ripple, the researcher only intends to use a simple rotor tooth shaping scheme, as prescribed in Somesan and Viorel (2013) [68] and Zhu et al (2008) [62], to improve the torque profile of the proposed machines. Meanwhile, the reader can refer to Chapters 3-6, where the torque ripple is imposed as a variable objective parameter, thus providing extended insights on its subjective use as constraint parameter elsewhere in this study.
} 
boundary limits of each design variable. As investigated in Ilhan et al (2012) [100], the RMS current density and current angle have been included as non-dimensional parameters so as to increase the flexibility of the optimum design considering FSMs are prone to saturation and magnetic crosscoupling effects. The flowchart outlined in Fig. 1.1 in section 1.5 describes the design optimisation procedure.

The optimisation is based on a non-gradient algorithm called the Non-dominated Sorting Genetic Algorithm II (NSGA-II). NSGA-II as an adaptive search technique inspired from nature, which works on the principle of Darwin's theory of survival-of-the-fittest, and broadly referred to as evolutionary algorithm. It works with a set of solutions (population) and as the simulation (evolution) proceeds, the individuals (solution) in the population improve, Deb et al: 2002 [107]. On this premise, each optimisation problem is ascribed 20 individuals while 100 iterations are applied, with the tuneable crossover and mutation index set at 20 and 10 , respectively.

\subsection{Results and Validation}

\subsubsection{Comparison and Discussions}

The simulation results of the optimisation problems executed for the three different drivetrain solutions are presented in Fig. 2.3, showing scatter plots of the optimal designs. It is clear that relatively less amount of PM is required to achieve the average torque requirements in the LS drivetrain, but with significant increase in the generator active mass. In reality, what is lost in terms of PM amount is traded off for an increase in the amount of copper and steel.

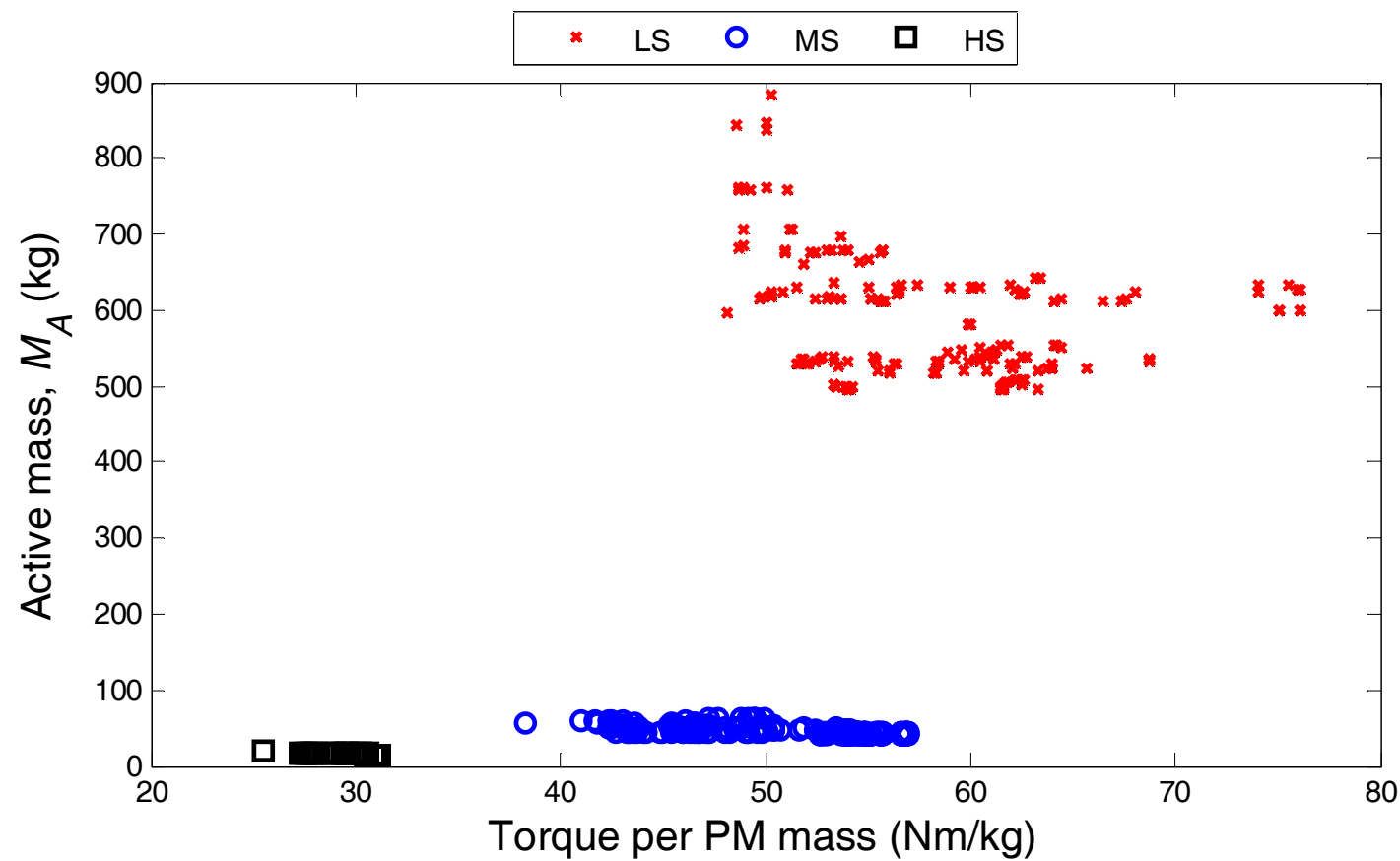

Fig. 2.3. Optimal design candidates presented for each PM-FSM drivetrain solution. 
As for the HS designs, the ratio of the torque requirement to PM mass is highest, with smallest amount of copper and steel is used. As expected, the geared MS designs presented a tradeoff with impressively high torque/PM densities similar to the LS designs at very low active mass as obtained in the HS design candidates.

Furthermore, the average values of the active mass for each optimal drivetrain design candidates are plotted as shown in Fig. 2.4. As indicated, the average active mass for the LS designs is highest compared to both the HS and MS options, given that all machines were designed for the same power levels of about $10 \mathrm{~kW}$. Thus, it is perceived that the potential incorporation of gearboxes in both the MS and HS designs, gave rise to suppression of their corresponding generator sizes. But considering the generator costs, it is clearly noted that the penchant for best $\mathrm{CoE}$ is escalated in the MS drivetrain because which unlike the HS option, its PM utilisation factor is more attractive.

To further evaluate the performance versus $\mathrm{CoE}$, a representative design is benchmarked from each optimal drivetrain solutions, with the baseline power requirement set within the $10 \mathrm{~kW}$ limit. The key performance indices for each drivetrain solution are displayed as shown in Table 2.2, leading to further conclusions.

As observed in Table 2.2, the torque-mass densities have similar trends with respect to the torque-PM mass densities. Moreover, it is clearly shown that the generator costs are proportional to the output torque. Hence, for the estimated total material costs, the LS generator is over 10 times the cost of the MS generator and 27 times that of the HS generator. However, between the MS and HS generators, the cost margin only differs by $250 \%$.

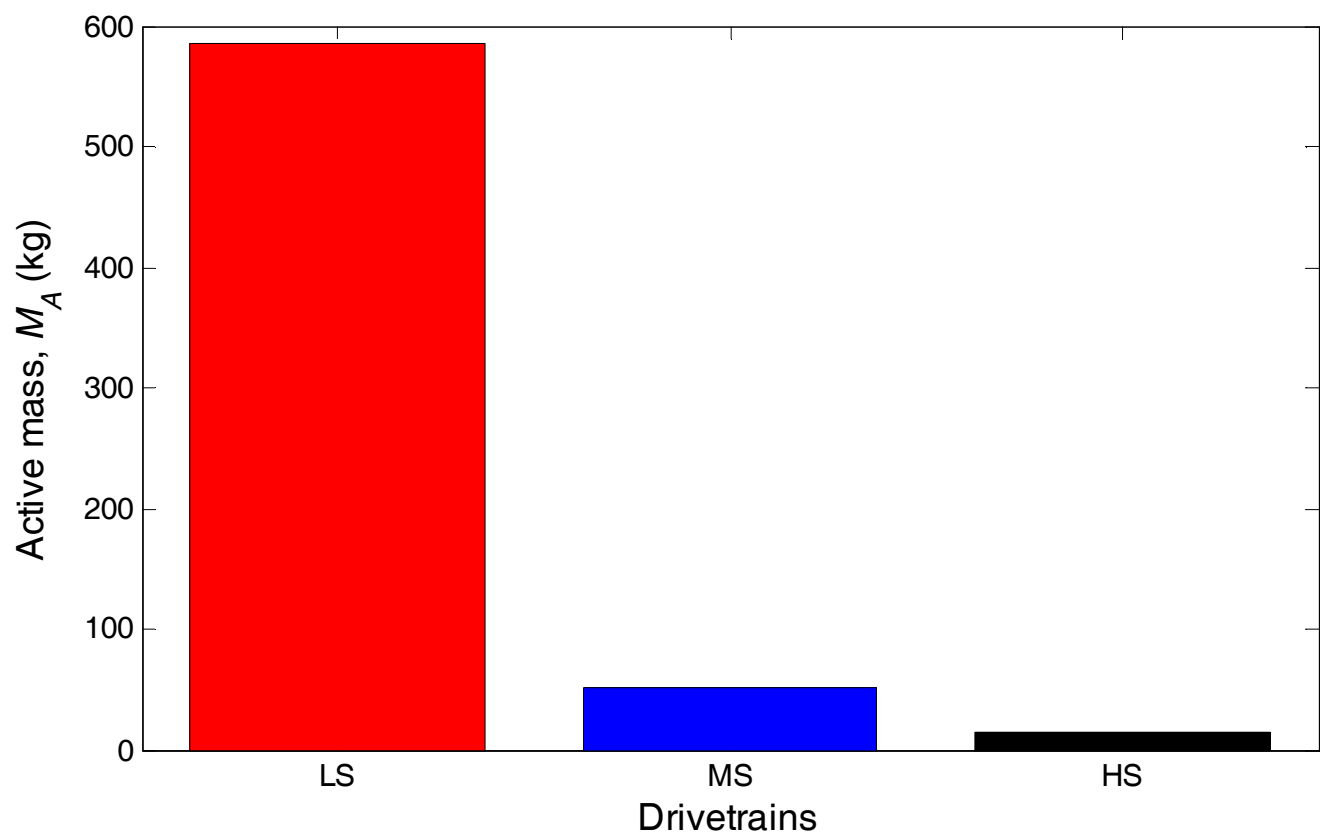

Fig. 2.4. Average generator mass for different PM-FSM drivetrains. 
Table 2.2. Comparison of PM-FSM performance parameters under different drivetrains at $10 \mathrm{~kW}$

\begin{tabular}{c|c|c|c|c}
\hline \multirow{2}{*}{ Parameters } & \multirow{2}{*}{ Units } & \multicolumn{3}{|c}{ Drivetrains } \\
\cline { 3 - 5 } & & LS & MS & HS \\
\hline Torque, $\tau_{e}$ & $\mathrm{Nm}$ & 3207.17 & 260.47 & 62.33 \\
Torque ripple, $\kappa_{\delta}$ & $\%$ & 10.29 & 9.96 & 10.28 \\
Output power, $\mathrm{P}_{\text {out }}$ & $\mathrm{kW}$ & 10.24 & 10.03 & 10.04 \\
Copper loss, $P_{C u}$ & $\mathrm{~kW}$ & 1.13 & 0.42 & 0.16 \\
Core loss, $P_{C o r e}$ & $\mathrm{~kW}$ & 0.02 & 0.09 & 0.26 \\
Efficiency, $\eta$ & $\%$ & 90 & 95.12 & 95.95 \\
Power factor, $\mathrm{PF}$ & - & 0.89 & 0.8 & 0.83 \\
Stator steel mass, $M_{F e S}$ & $\mathrm{~kg}$ & 292.56 & 22.88 & 7.66 \\
Rotor steel mass, $M_{F e R}$ & $\mathrm{~kg}$ & 91.04 & 8.03 & 2.23 \\
Copper mass, $M_{C u}$ & $\mathrm{~kg}$ & 58.64 & 6.15 & 2.52 \\
PM mass, $M_{P M}$ & $\mathrm{~kg}$ & 52.08 & 4.75 & 1.99 \\
Active mass, $M_{A}$ & $\mathrm{~kg}$ & 494.32 & 41.82 & 14.40 \\
Torque/active mass & $\mathrm{Nm} / \mathrm{kg}$ & 6.49 & 6.23 & 4.33 \\
Torque/PM mass & $\mathrm{Nm} / \mathrm{kg}$ & 61.58 & 54.83 & 31.32 \\
Total material cost ${ }^{14}$ & $\mathrm{USD}$ & 4625.48 & 421.88 & 169.38 \\
Split ratio, $\Lambda_{0}$ & - & 0.63 & 0.57 & 0.53 \\
Aspect ratio, $\kappa_{L}$ & - & 0.68 & 0.69 & 0.83 \\
Current density, $\mathrm{J}$ & $\mathrm{A} / \mathrm{mm}$ & 2.673 & 4.999 & 4.999 \\
\hline
\end{tabular}

Consequently, should a gearbox cost ratio of $183 \%$ between the three-stage HS gearbox and single-stage MS gearbox as implied in Polinder et al (2006) [11] be adopted, then the MS generator drivetrain costs is improved to $136 \%$. But with higher operation and maintenance costs accruing to the HS gearbox, the cost of the MS system is anticipated to improve further.

Also, comparing the power factor shows the LS design with the best outcome at 0.89 ; however, the power factor limit was achieved in all three drivetrains. The excellent power factor in the LS design is possible only because it incurred the lowest current density after the MDO process. To account for this, the current densities of the different drivetrain solutions evaluated in the MDO global search space are fitted against power factor as shown in Fig. 2.5, with highlights made in the different regions where the selected benchmarks are sited. To this end, the LS benchmark potentially yields the cheapest SSC due to its excellent power factor compared to the rest, because, according to Sulaiman, Kosaka and Matsui (2012) [95], the conduction loss of SSCs depends on power factor, which escalates their power ratings and costs. But such cost rebate might consequently be diminished considering its oversized generator, with its implications for very high installation and logistics costs. Furthermore, the power factor of the LS design improved because of the similar airgap length imposed on the three designs, which capacity to improve the magnetising reactance in the resulting oversized generator. To support this claim, it is reported in Pyrhönen, Jokinen and Hrabovcova (2008) [96] that

\footnotetext{
14 The cost estimations are performed based on quotations in US dollars (USD) mined from Fasolo, Alberti and Bianchi (2014) [69].
} 
by decreasing the magnetic inductance of machine, greater amount of reactive current is usually generated to the detriment of the power factor. Considering the positive correlation between efficiency and power factor observed for all three PM-FSM drivetrains in Fig. 2.6, the possibility arises for the $10 \mathrm{~kW}$ LS PM-FSM to achieve unitary power factor at less than $95 \%$ machine efficiency.
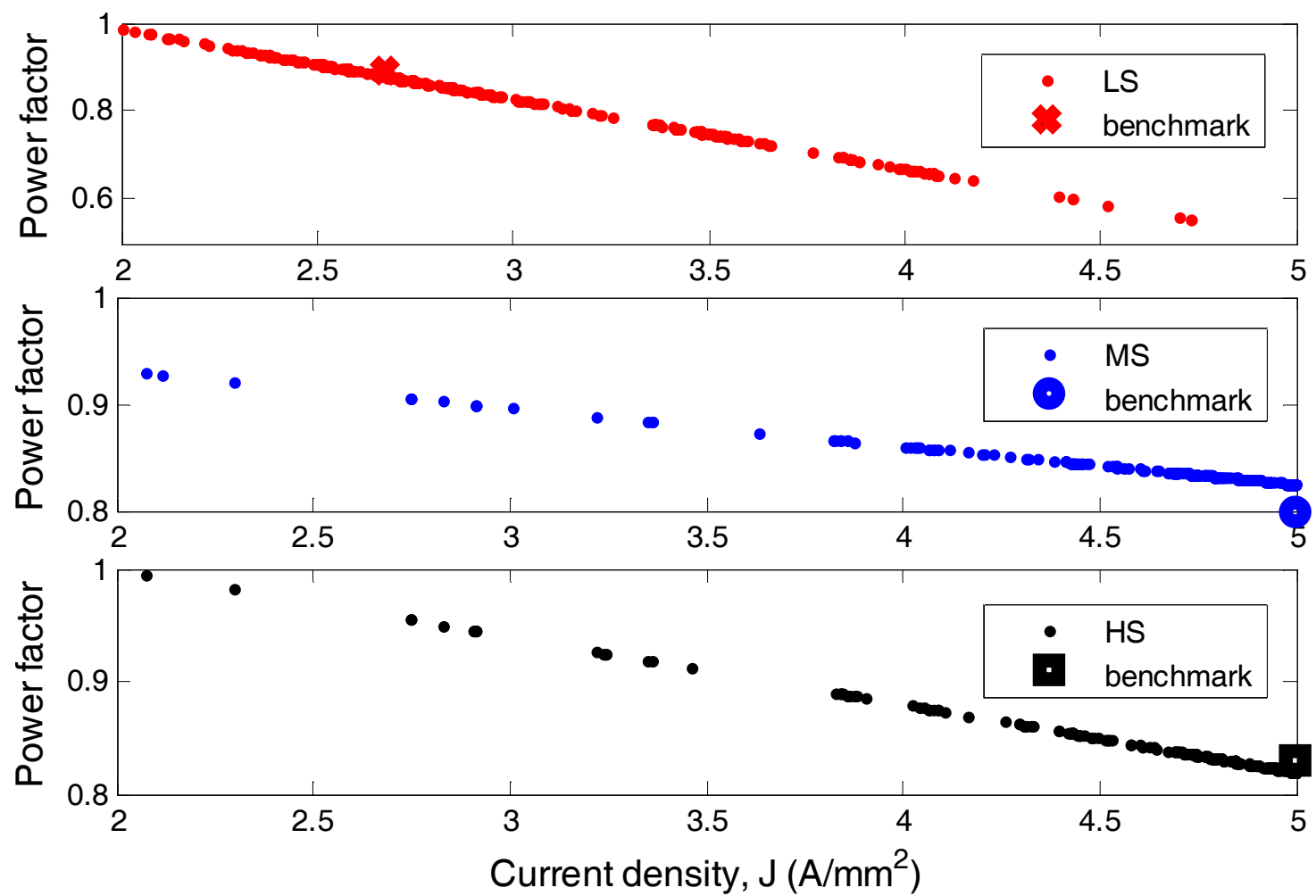

Fig. 2.5. Response of current density to power factor evaluated for different PM-FSM drivetrains.
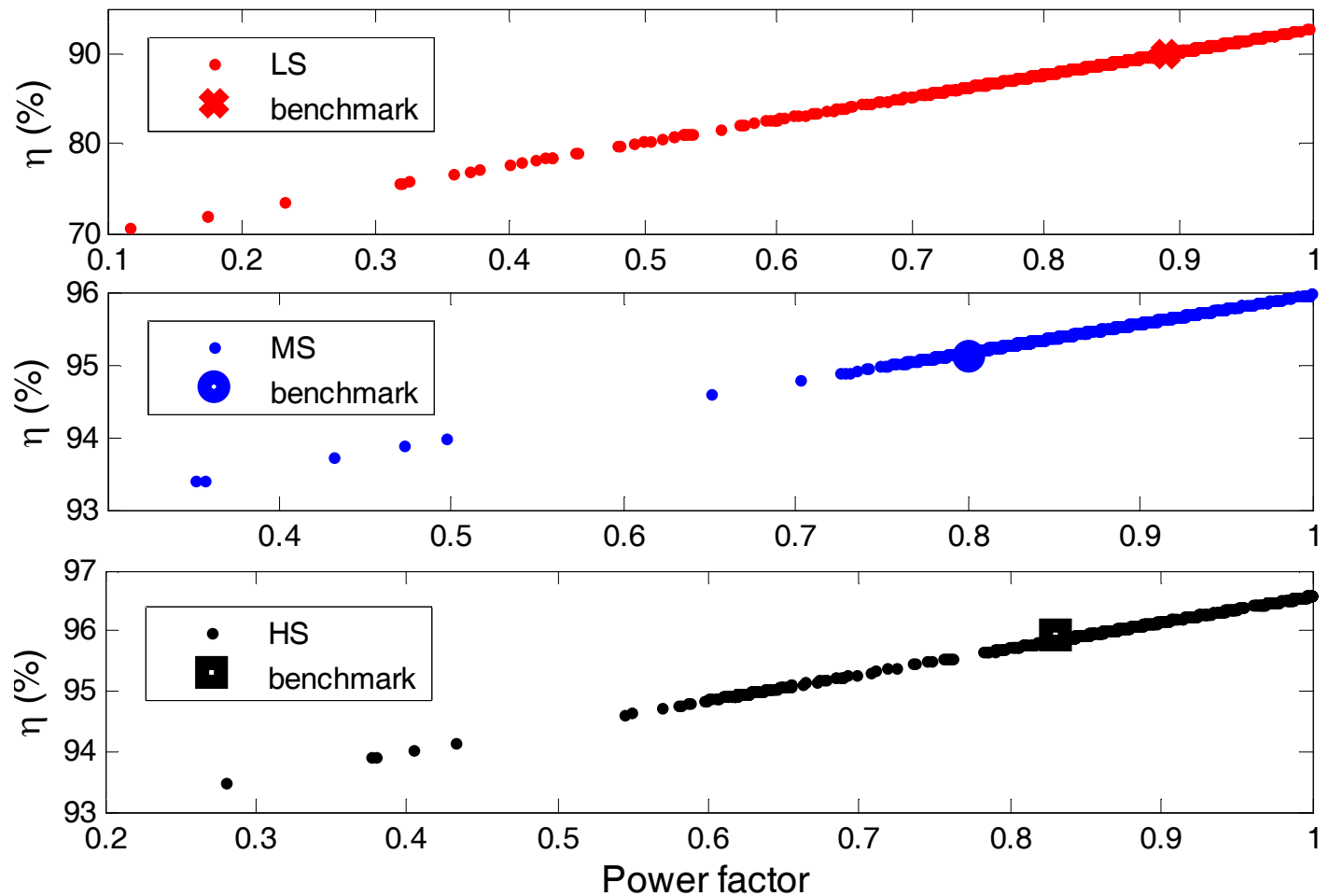

Fig. 2.6. Efficiency versus power factor evaluated for the different optimal PM-FSM drivetrains. 

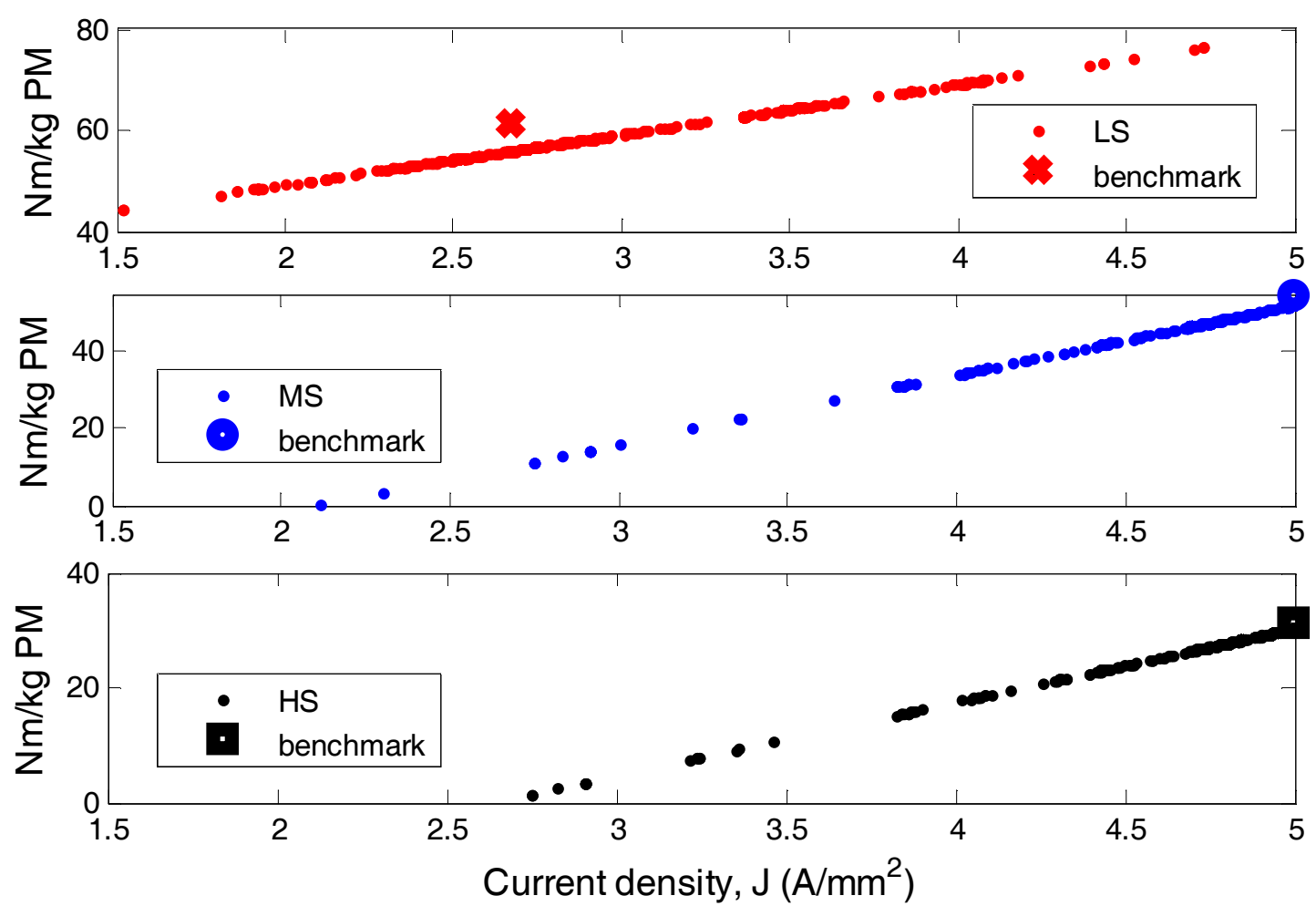

Fig. 2.7. PM utilisation factor versus current density evaluated for different PM-FSM drivetrains.

Meanwhile, due to the torque capability expressed in the LS design, one would expect greater torque per PM mass density as against the published value in Table 2.2. But as observed in Fig. 2.3, a good proportion of the evolved torque/PM mass in the LS designs were mostly overlapped by those of the MS designs, no thanks to high PM usage and low current densities, proven by theoretically fitting the current density operated during the MDO process as shown in Fig. 2.7.

On the other hand, the copper loss in the LS design dominates among the three different drivetrains as observed in Table 2.2. By fitting all the control variables used for the MDO process, it is not difficult to infer the reason for this. In Fig. 2.8, it is clearly shown that the characteristics of the current density in the LS design are different from the rest. Whereby high current density is desired in the MS and HS designs for improved machine efficiency, the reverse appears to be the case for the LS design. Evaluation of the RMS current values for the reported benchmarks in Table 2.2 resulted in 24.91 A, 12.24 A and 6.54 A for the LS, MS and HS designs, respectively. Thus, by reducing the current density of the LS design, the phase current value decreases, viz., $I_{S}^{2} R_{S}$ loss. For this to happen, it is clearly portrayed in Fig. 2.9 that a reduction in the optimal split ratio is also necessary.

In respect of the core losses, the HS design experiences the highest amount among the three drivetrains as shown in Table 2.2, due to its highest fundamental frequency. Notwithstanding, this increment is nothing compared to that of the copper loss observed in the LS design, which apparently resulted in the worst efficiency performance of the latter, barely surviving the $90 \%$ design threshold. 
Meanwhile, as also indicated, those of the MS and HS machines were logged in excess of $95 \%$. As for torque ripple values, it is observed that the variation among the three drivetrains is not dramatic, also bearing in mind that each drivetrain solution satisfied the design requirements.
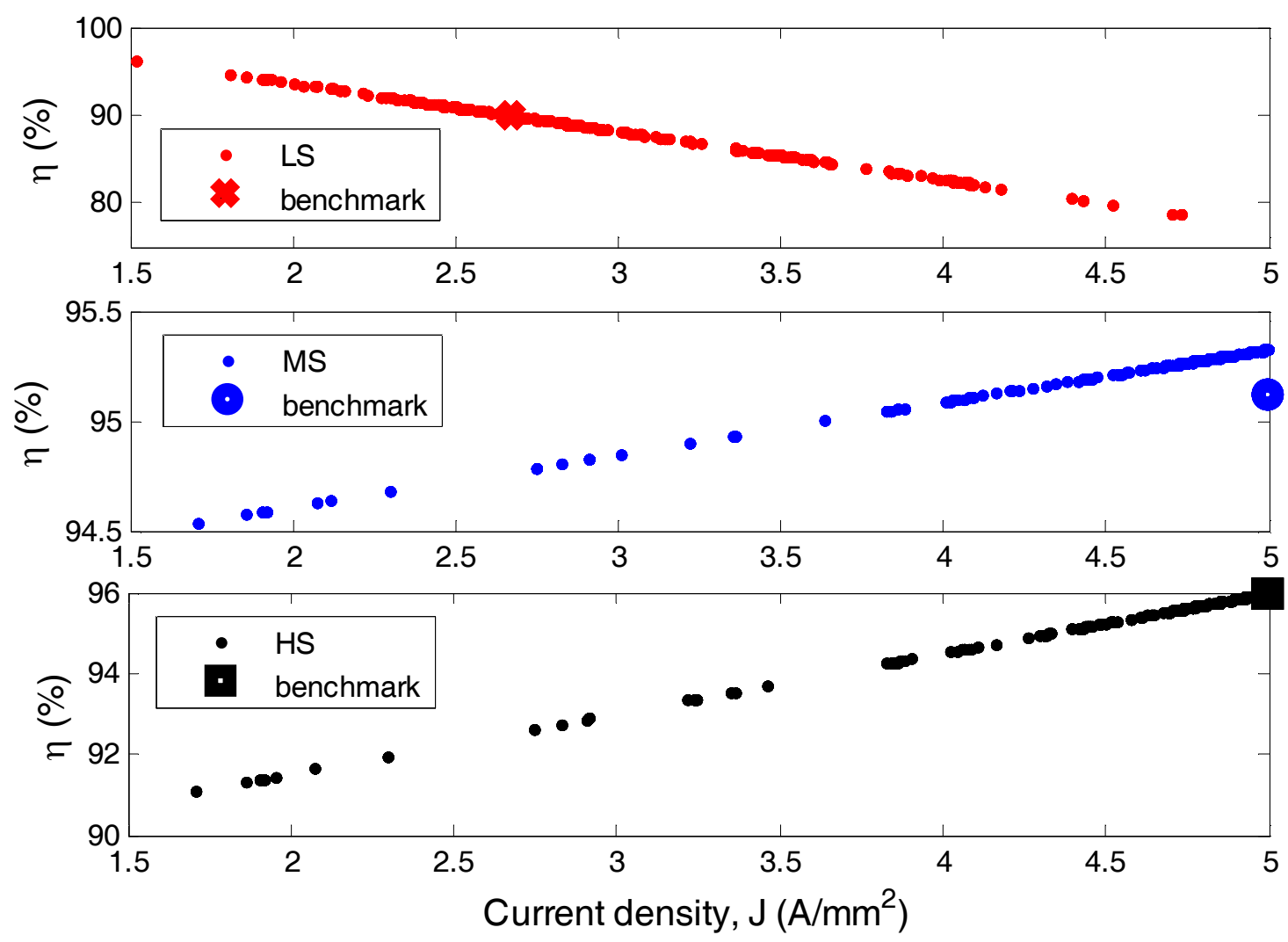

Fig. 2.8. Efficiency versus current density evaluated for different PM-FSM drivetrains.
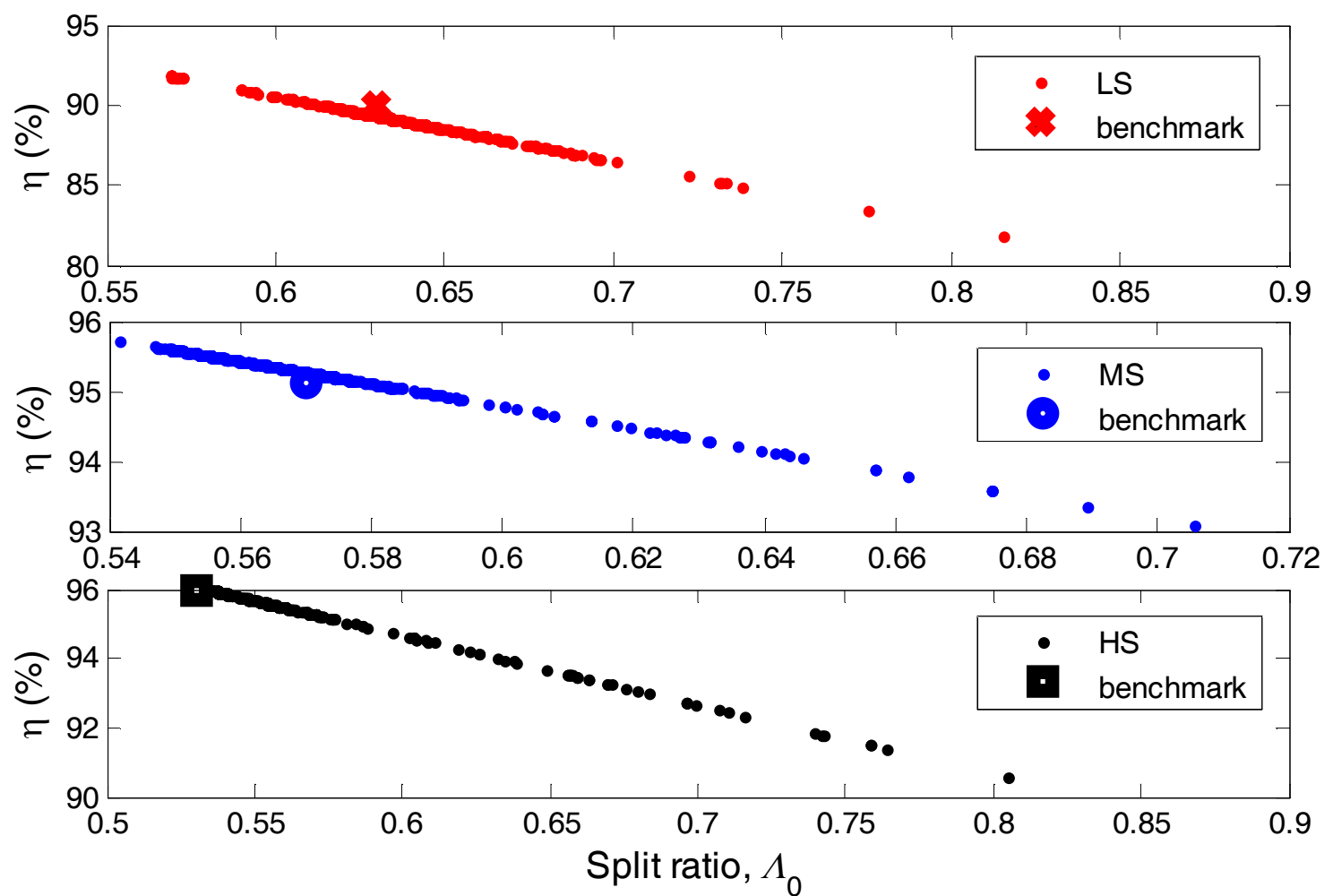

Fig. 2.9. Efficiency versus optimal split ratios evaluated for different PM-FSM drivetrains. 
While it may not be a conclusive task to predict which of the generators give the best drivetrain solution, the MS design is notwithstanding acknowledged as the preferred solution due to yielding the best compromise between $\mathrm{CoE}$ and performance. To reach this conclusion, the following are noteworthy:

- The torque densities (both of the PM and the total active mass) of the MS design is as high as those observed in the LS designs, while their costs remain comparable to that of the HS designs. This is beneficial to provide limited size and cost of the wind turbine tower and hub.

- Although the power factor is lowest for the MS generator, perhaps, due to very high current densities, which increases the leakage reactance and may in turn increase the losses and cost of the SSC. However, the higher cost implications of the LS generator and the HS gearbox, in any case, diminish their SSC cost advantage occasioned by better power factor.

- Lastly, considering the baseline power, the MS generator produced comparable torque ripple values and generator efficiency with overriding impact on the drivetrain reliability and energy yield.

\subsubsection{3-D FEA Validation}

In this subsection, the design feasibility of the 2-D static FEA predictions, are compared in 3-D transient FEA. The MS benchmark design selected in the preceding subsection is primed for the analyses. The magnetic field distributions realised 3-D model is as shown in Fig. 2.10, while the 2-D and 3-D FEA calculated terminal voltage waveforms are compared in Fig. 2.11. As can be seen, a good agreement has been obtained. Loose meshing in the models constituted in 3-D FEA is likely responsible for the rough edges observed in its corresponding voltage waveform.

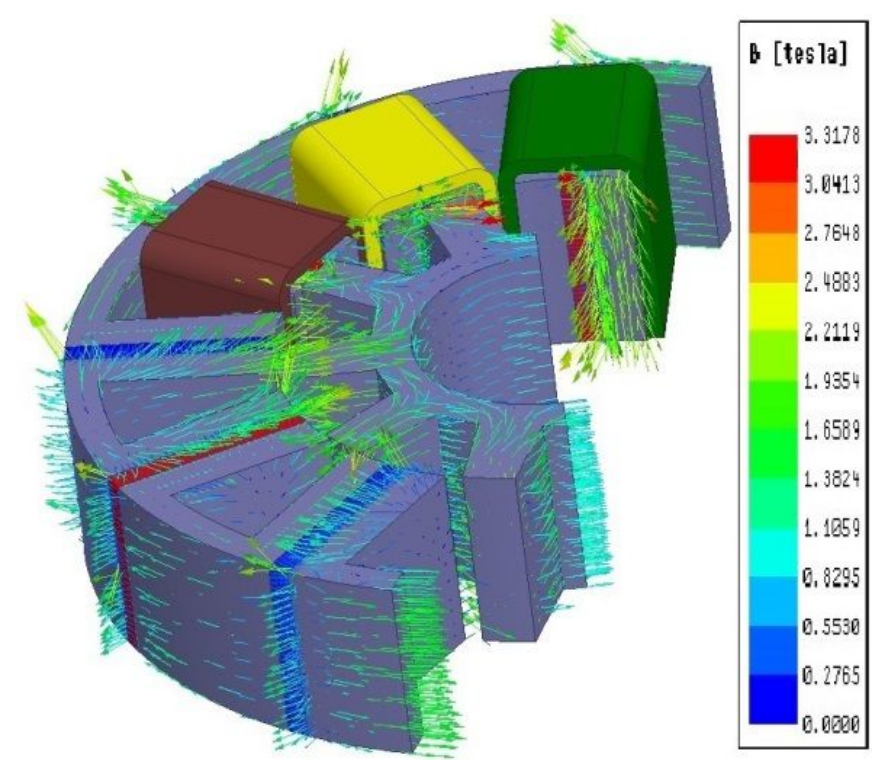

Fig. 2.10. 3-D FEA model showing the magnetic flux lines at rated condition. 


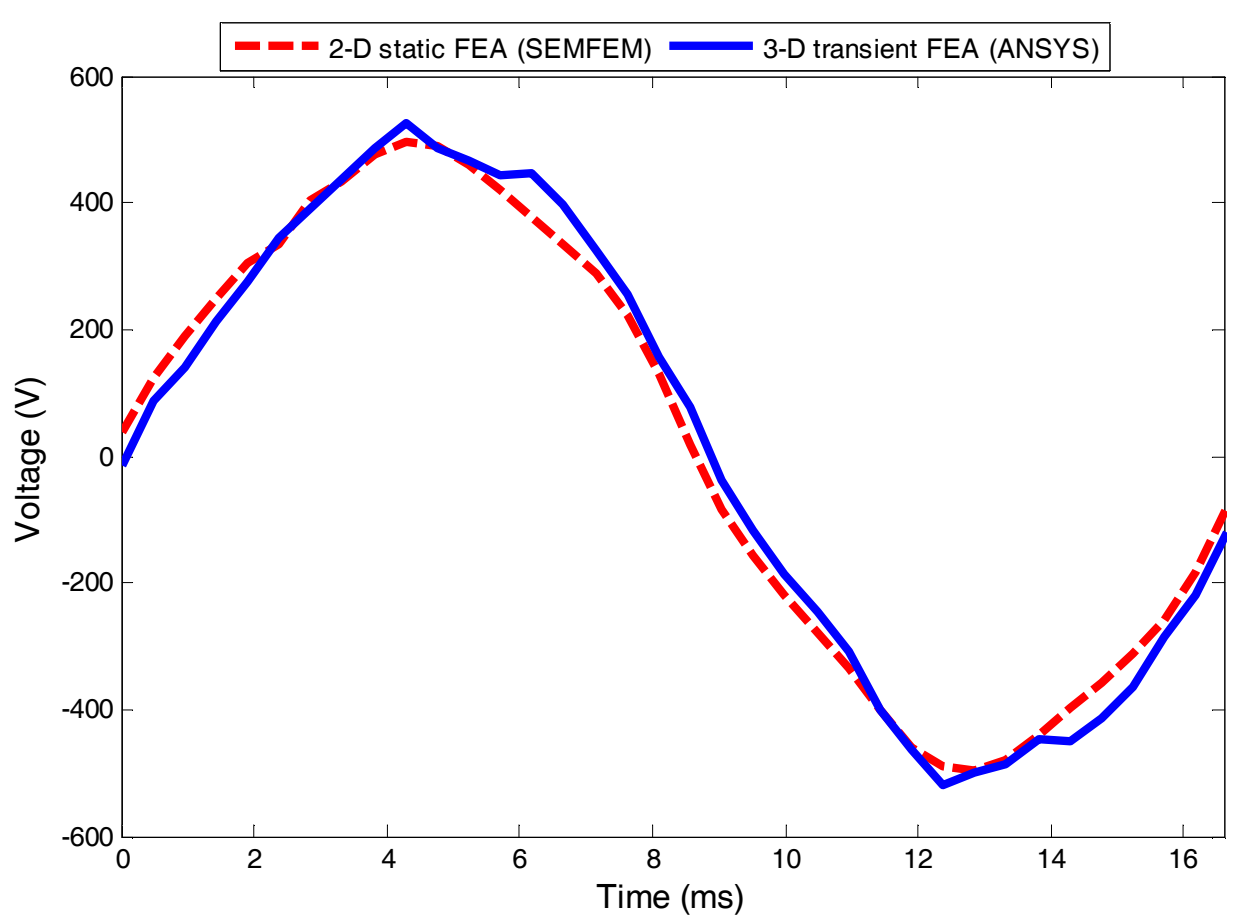

Fig. 2.11. Comparison of rated phase voltages at $360 \mathrm{r} / \mathrm{min}$.

\subsection{Chapter Summary}

In this chapter, the drivetrain comparison of 12/10 PM-FSM for small power $(10 \mathrm{~kW})$ wind generators has been studied using FEA-based design optimisation. The evaluation is anchored on CoE versus performance for each generator drivetrain - LS, MS and HS. The results show that, in terms of costs, the HS drivetrain presents the cheapest design but at a very high PM utilisation rate - torque per PM mass at less than $35 \mathrm{Nm} / \mathrm{kg}$. In addition, the cost of HS drivetrains is escalated due to potentially highest gearbox installation and maintenance costs.

On the other hand, the LS drivetrain uses, relatively, the lowest amount of PM material, at a torque per PM mass ranging from $47 \mathrm{Nm} / \mathrm{kg}$ to $67 \mathrm{Nm} / \mathrm{kg}$, but at the expense of producing an oversized generator, thus increasing the active material cost, to at least, 10 times the cost of the MS generator and 27 times that of the HS generator. In addition to this, very large LS generators mean that the cost of installation and other logistics are further stretched in proportionally sizing the wind turbine tower. To this end, the MS design is nominated as the preferred solution because of apparent tradeoffs in the torque-PM densities $(37-57 \mathrm{Nm} / \mathrm{kg})$ and generator costs.

Based on some optimal benchmarks from each of the three drivetrain solutions, it is observed that the huge size of the LS generator requires very high amount of copper, such that a resulting high copper loss limits the efficiency target to the advantage of the power factor. On the other hand, the efficiency requirements of the MS and HS designs are both exceeded, i.e., beyond $95 \%$, with their power factor also respected for the design targets. 
With the electromagnetic design of the 12/10 PM-FSM wind generator as the main interest in the drivetrain comparison, the MS solution, nevertheless, provides an ideal solution among the three drivetrains. However, it has to be said that the study is limited because, apart from the evaluated costs of the optimally designed generators, the cost of other drivetrains components such as gearboxes and SSCs were considered merely as estimates. 


\section{Chapter Three}

\section{Design Optimisation and Evaluation of PM-FSMs}

This chapter is used to compare and highlight the adaptability of the 12/10 and 12/14 rare-earth PMFSM for MS wind generator drives. Once again, the evaluations are carried in 2-D static FEA calculations together with design optimisation. The approach which is on non-gradient-based multiobjective design optimisation (MDO) strategy, also gives insight into the evolutionary performance from small-scale kW to multi-MW designs. In the end, some of the obtained results are confirmed in corresponding 3-D FEA evaluations. Already, parts of this chapter have been discussed as a conference paper in Akuru and Kamper (2015) [101].

\subsection{Introduction}

Based on increasing demand for large megawatts wind turbine systems, the need to reduce the cost of generation is becoming critical such that attention is now being directed towards the available wind generator drivetrains. The known wind generator drivetrains are low-speed (LS), medium-speed (MS) and high-speed (HS) drives. A summary of the different drivetrain characteristics is already presented in Table 1.3 in subsection 1.3.2, wherein it shown that MS drivetrains yields a good compromise to LS and HS systems.

As underscored in Schmidt and Vath (2012) [20], MS drivetrains produce greater annual energy yield per generator cost at average wind speeds. This is equally elucidated in the preceding chapter of this thesis (Chapter 2), whereby 12/10 PM-FSMs which were optimised and compared in different wind generator drivetrains showed the MS drivetrain as the best solution in terms of producing high torque densities at low generator costs.

PM-FSMs, which are not entirely new, belong to a class of stator-active machines with double saliency, Zhang et al: 2009 [45]. The operation of PM-FSMs is such that it produces bipolar flux in the armature (stator) teeth as a result of modulation of the flux linkage with respect to change in rotor position (flux switching). As previously noted, the field excitation system can be equally be either wound-fields (WFs) based on appropriate configurations, Zhu et al: 2015 [102]. Besides, PM-FSMs are endowed with such qualities as high power (torque) density, good thermal dissipation, and a robust rotor structure. In addition, concentrated windings, similar to those in SRMs are employed, leading to low end-winding length and increased efficiency. However, it must be said that the torque pulsations in FSMs are known to be very severe, Hua et al: 2008 [46]. 
Just like in typical PM synchronous machines, PM-FSMs are also designed as axial flux (with disc type rotors), transverse flux (with pancake or disc type rotors) or radial flux (with cylindrical rotors). Oftentimes, radial-flux PM-FSMs have been applied in in-wheel traction for EV/HEV motor operation at low power levels, Somesan and Viorel: 2013 [68], Zhu et al: 2015 [104] and Cao, Mi and Cheng: 2012 [105].

In terms of wind generator applications, transverse-flux and axial-flux PM-FSMs have been more or less popular in LS drivetrains, at low power levels, Hao et al: 2012 [73], Lin et al: 2011 [74], Dobzhanskyi et al: 2012 [75] and Yan et al: 2009 [76]. However, an exception is in Wang et al (2013) [81], whereby a high-temperature superconductor (HTS) radial-flux WF-FSM with highpower density is proposed to replace its PM-FSM counterpart. But unlike the radial-flux designs, transverse-flux and axial-flux PM-FSMs manifest in very complex structures, which are bound to increase their manufacturing and assembly costs. Besides, LS systems are less attractive because they result in heavier and more expensive generators which may also implicate the cost of the overall wind turbine architecture.

With regards to HS systems, not much is documented for PM-FSMs designed as wind generators. In Yu and Niu (2015) [79], a new single-phase magnetless FSM was developed for rooftop WPG with rated power of less than $1 \mathrm{~kW}$. Also, Thomas, Zhu and Jewell (2011) [80] demonstrated the feasibility of PM-FSMs for so-called high-speed and high power $(50 \mathrm{~kW})$ generator application. The known issue with HS systems is their very high gearbox reliability issues, but apart from this, PM-FSMs when designed for HS drivetrains are prone to experience increased core losses as revealed in Akuru and Kamper (2015) [98].

Coming to MS drivetrains, the application of radial-flux PM-FSM to geared MS wind generator drives was never emphasised by other researchers in the literature until the current study. Meanwhile, Anyuan et al (2009) [106] studied and compared the 12/10 and 12/14 PM-FSM based on parameter variation at $400 \mathrm{r} / \mathrm{min}, 1.5 \mathrm{~kW}$ power, without reference to any application requirements. In the study, a higher average torque and lower torque ripple performance of the 12/14 over the 12/10 were expounded. On the other hand, the limitation of using gradient-based technique in the design optimisation and comparison of both PM-FSM topologies was studied by Akuru and Kamper (2015) [101], showing that such optimisation process results in hard problems in their nonlinear multimodal search space.

Therefore, it goes without saying that, for a more robust optimisation, the use of metaheuristic optimisation algorithms in the design of PM-FSMs cannot be overemphasized. Moreover, because existing studies concentrated on the analyses of small $\mathrm{kW}$ power ratings, which may not be repre- 
sentative of the power levels in industrial-scale wind turbines, there is need to investigate the performance of FSMs when it is scaled from $\mathrm{kW}$ to MW power levels. Thus, this chapter is based on the multi-objective design optimisation (MDO) and design comparison of the 12/10 and 12/14 rare-earth PM-FSMs proposed for geared MS wind generator drives, and evaluated at $10 \mathrm{~kW}$ and $3 \mathrm{MW}$ power levels. The main focus here is to uncover tradeoffs which may occur in rare-earth PM-FSMs designed at the prescribed power levels.

An illustration of the proposed drivetrain topology is already depicted in Fig. 1.12, subsection 1.3.3. Because the torque density is critical to the generator, which in turn defines the nacelle weight, a reduced generator mass will be solely pursued in the MDO process escalated in this chapter. Also to be considered, is the torque ripple, which if unusually high, can be hazardous to the MS gearbox leading to mechanical vibrations. Of course, other important penalties such as high power factor and efficiency are bound to increase the drivetrain efficiency; these performance variables will also be carefully considered.

\subsection{Model Creation}

The layouts for the three-phase 12/10 and 12/14 PM-FSM result in balanced magnetic fields. However, the 12/14 topology, with a higher fundamental frequency, has its phase "B" and "C" coils swapped compared to the 12/10 design as shown in Fig. 3.1. For the same operating conditions, the $12 / 14$ produces higher fundamental frequency, torque and induced phase voltages than the 12/10 machine based on the following expressions:

$$
\begin{aligned}
f_{e} & =\frac{n_{s} N_{r}}{60}, \\
\tau_{e} & =\frac{3}{2} N_{r}\left(I_{q} \lambda_{M}+I_{d} I_{q}\left(L_{d}-L_{q}\right)\right), \\
E_{g} & =2 \pi f_{e} N_{t} \kappa_{e} \dot{B}_{g} l_{s t} \tau_{s} c_{s},
\end{aligned}
$$

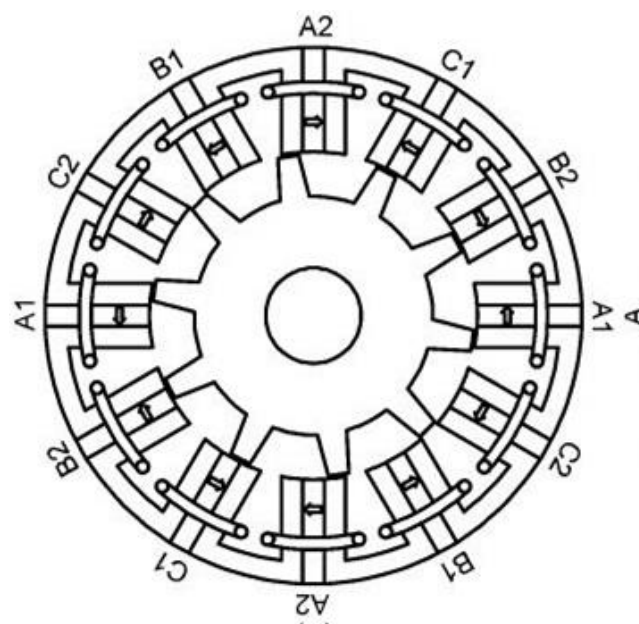

(a)

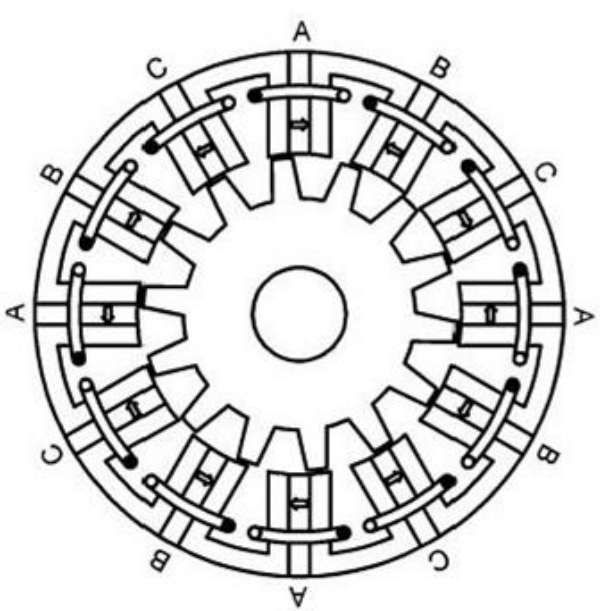

(b)

Fig. 3.1. All poles wound PM-FSM: (a) 12/10 design, and (b) 12/14 design. 
Table 3.1. Design specifications

\begin{tabular}{c|c|c|c}
\hline Symbol & Parameters & $\mathbf{1 0 ~ k W}$ & $\mathbf{3 ~ M W}$ \\
\hline \hline$n_{s}$ & Rated speed & $360 \mathrm{r} / \mathrm{min}$ \\
$\mathrm{J}$ & Current density & $1-5 \mathrm{~A} / \mathrm{mm}^{2}$ \\
$\theta_{\mathrm{sF}}$ & Slot fill factor & \multicolumn{2}{|c}{0.4} \\
$B_{r}$ & PM remanence & \multicolumn{2}{|c}{$1.2 \mathrm{~T}$} \\
$\mathrm{PF}$ & Power factor & \multicolumn{2}{|c}{$\geq 0.8$} \\
$\eta$ & Efficiency & $\geq 90 \%$ & $\geq 97 \%$ \\
$g$ & Airgap length & $0.7 \mathrm{~mm}$ & $2.5-3 \mathrm{~mm}$ \\
\hline
\end{tabular}

where $n_{s}$ is the mechanical speed in $\mathrm{r} / \mathrm{min}, N_{r}$ is the number of rotor poles, $\lambda_{M}$ is the no-load flux linkage, $I_{d}$ and $I_{q}$ are the dq axes phase currents, $L_{d}$ and $L_{q}$ are the dq axes inductances, $N_{t}$ is the number of turns per phase, $\kappa_{e}$ is a factor to account for some leakage, $\dot{B}_{g}$ is the peak airgap flux density, $l_{s t}$ is the stack length of the lamination cores, $\tau_{s}$ is stator pole pitch, and $c_{s}$ is the stator tooth arc factor.

The initial geometries of the proposed PM-FSM topologies are generated from the design specifications imposed on the $10 \mathrm{~kW}$ and $3 \mathrm{MW}$ designs as given in Table 3.1. With this information, the PM-FSMs are modelled according to the sizing-design procedure in Appendix A1.2. Based on the design data in Table 3.1, the conceptual stator outer diameter of the 12/10 machines are drawn. For a fair comparison, the same stator outer diameter is retained a priori for the 12/14 machines.

\subsection{FEA Multi-Objective Design Optimisation}

No doubt, the design optimisation of PM-FSMs presents a nonlinear multi-objective problem. Thus, the best optimisation procedure is propagated when the problem is constrained and a multi-objective design is pursued. In the present scenario, where wind generator design is implied, certain key performance indices, such as minimum mass and torque ripple, are simultaneously optimised in order to increase the wind turbine cost savings and improve the drivetrain reliability, as well as declare any other potential tradeoffs.

The approach taken for the MDO is to concurrently minimise the active mass $\left(M_{A}\right)$ and torque ripple $\left(\kappa_{\delta}=\frac{\tau_{e(\max )}-\tau_{e(\min )}}{\tau_{e}}\right)$ while constraining output power $\left(\mathrm{P}_{\text {out }}\right)$, efficiency $(\eta)$ and power factor $(\mathrm{PF})$, with $\tau_{e}$ as defined previously, $\tau_{e(\min )}$ and $\tau_{e(\max )}$ represents the instantaneous minimum and maximum peaks, respectively. The objective functions are proposed in two combinatorial problems, weighted equally. Thus, two different constraints are evinced because of the two different power levels being investigated. The MDO problems are summarised as follows:

Minimise $\quad F(\bar{x})=\left[\begin{array}{ll}M_{A} & \kappa_{\delta}\end{array}\right]^{T}$,

with boundary constraints on $n$ number of design parameters defined as 


$$
x_{i}^{(L)} \leq x_{i} \leq x_{i}^{(U)}, \quad i=1, \ldots, n,
$$

subject to inequality constraints on the design requirements such as

$$
\begin{aligned}
& G_{1}(\bar{x})=\left[\begin{array}{lll}
\mathrm{P}_{\text {out }} \geq 10 \mathrm{~kW} & \eta \geq 90 \% & \mathrm{PF} \geq 0.8
\end{array}\right]^{T}, \\
& G_{2}(\bar{x})=\left[\begin{array}{lll}
\mathrm{P}_{\text {out }} \geq 3 \mathrm{MW} & \eta \geq 97 \% & \mathrm{PF} \geq 0.8
\end{array}\right]^{T},
\end{aligned}
$$

where $\bar{x}$ is the vector of design parameters, $T$ is a symbol for vector transposition, $L$ and $U$ are insignias to specify the lower and upper boundary limits as previously noted, $n$ is total the number of design parameters, and $G_{1}$ and $G_{2}$ are the inequality constraint vectors for $10 \mathrm{~kW}$ and $3 \mathrm{MW}$ design requirements, respectively.

A total of 12 and 13 design parameters are varied for the $10 \mathrm{~kW}$ and $3 \mathrm{MW}$ machines, respectively. Detailed information about the design parameters is provided in Table 3.2, while the optimisation procedure is set-up as shown in Fig. 1.1 in section 1.5 in Chapter 1. Based on some random starting population of the design variables, a conceptual model is constructed and analysed in 2-D FEA, through an optimiser which processes the problems until the convergence criteria are achieved.

For the constrained MDO problems, a metaheuristic evolutionary algorithm-the Nondominated Sorting Genetic Algorithm II (NSGA-II) — proclaimed as "fast and elitist" is adopted, Deb et al: 2002 [107]. NSGA-II has been applied to: IMs in Besnerais et al (2008) [108], IPMs in Jannot et al (2011) [109] and brushless DFIGs in Wang et al (2016) [110] as MDO problems, but not yet for PM-FSMs to the researcher's best knowledge. The advantage of using this algorithm for MDO problems is that it produces better spread of solutions to the truest convergence of the Pareto optimal front, compared to other evolutionary algorithms. However, because they require greater function evaluations for convergence, they are very time consuming compared to deterministic methods.

NSGA-II operates on the basis of certain genetic operators - mutation and crossover - that are applied to existing members of the population so as to evolve into new solutions. The populations keep evolving based on the prescribed boundaries, constraints and objective functions, until the allocated number of generations are exhausted and the simulation is terminated. For the professed MDO problems in (3.4)-(3.7), the NSGA-II parameters have been set forth as shown in Table 3.3. The difference observed in the mutation probability is due to the different number of design variables considered for each MDO problem category indicated.

\subsection{Implementation and Optimisation Results}

The constrained MDO problems are implemented for the 12/10 and 12/14 machines, at two different power levels- $10 \mathrm{~kW}$ and $3 \mathrm{MW}$. To ensure a fair comparison, the same boundary conditions and 
parameters settings are maintained as indicated in Tables 3.2 and 3.3, respectively, for both the 12/10 and 12/14 machines. The average total time taken for the optimisation of the $10 \mathrm{~kW}$ designs is 37.4 hours at $29.6 \mathrm{~s}$ per FEA evaluation on an i7-4770 CPU workstation, while that of the $3 \mathrm{MW}$ designs cruise to around 65.4 hours at $75.6 \mathrm{~s}$ per FEA solution.

The optimisation results which evolved from the initial populations are evinced as shown in Figs. 3.2 and 3.3. It is clear from both figures that some amount of compromises persists between the active mass and torque ripple. As similarly reported in Anyuan et al (2009) [106] for a $1.5 \mathrm{~kW}$ design, it is observed that the competing variables perform better for the 12/14 machine compared to the $12 / 10$ machine at $10 \mathrm{~kW}$ power. However, in the present circumstance, it is observed that any advantage acquired for the 12/14 in a small $10 \mathrm{~kW}$ machine is reversed at $3 \mathrm{MW}$ power, and vice versa. To articulate why this is the case, the researcher committed to further carefully investigate the behavior of the optimal design candidates at $3 \mathrm{MW}$ power based on some selected benchmarks.

Table 3.2. Boundary conditions of design parameters

\begin{tabular}{c|c|c|c|c|c}
\hline & \multirow{3}{*}{ PM-FSM design } \\
\cline { 3 - 6 } Design variables & $\overline{\boldsymbol{x}}$ & $\mathbf{1 0} \mathbf{k W}$ & \multicolumn{2}{|c}{$\mathbf{3} \mathbf{M W}$} \\
\cline { 3 - 6 } & & $\boldsymbol{x}^{(\boldsymbol{L})}$ & $\boldsymbol{x}^{(\boldsymbol{U})}$ & $\boldsymbol{x}^{(\boldsymbol{L})}$ & $\boldsymbol{x}^{(\boldsymbol{U})}$ \\
\hline Current angle (deg.) & $\alpha$ & 0 & 90 & 0 & 90 \\
Current density (A/mm (m) $^{2}$ & $\mathrm{~J}$ & 1 & 5 & 1 & 5 \\
Stator outer diameter (mm) & $D_{\text {out }}$ & 250 & 300 & 1550 & 1650 \\
Stator inner diameter (mm) & $D_{\text {in }}$ & 162 & 180 & 1150 & 1250 \\
Shaft diameter (mm) & $D_{\text {sh }}$ & 80 & 85 & 800 & 850 \\
Stack length (mm) & $l_{s t}$ & 90 & 180 & 600 & 1200 \\
Airgap length (mm) & $g^{15}$ & - & - & 2.5 & 3 \\
PM length (mm) & $b_{p m}$ & 5 & 10 & 35 & 70 \\
Rotor pole width (mm) & $b_{p r}$ & 9 & 18 & 70 & 140 \\
Slot opening width (mm) & $b_{s l s}$ & 7.5 & 15 & 40 & 80 \\
Stator yoke height (mm) & $h_{y s}$ & 7.5 & 15 & 37.5 & 75 \\
Rotor yoke height (mm) & $h_{y r}$ & 7.5 & 15 & 40 & 80 \\
Rotor tooth tapering factor & $t_{0}$ & 0.5 & 1 & 0.5 & 1 \\
\hline
\end{tabular}

Table 3.3. NSGA-II parameters

\begin{tabular}{c|c|c}
\hline \multirow{2}{*}{ Parameters } & \multicolumn{2}{|c}{ PM-FSM design } \\
\cline { 2 - 3 } & $\mathbf{1 0} \mathbf{~ k W}$ & $\mathbf{3 ~ M W}$ \\
\hline Mutation probability & 0.083 & 0.076 \\
Crossover probability & 0.9 & 0.9 \\
Mutation distribution index & 10 & 10 \\
Crossover distribution index & 20 & 20 \\
Population size & 25 & 25 \\
Iterations & 100 & 100 \\
\hline
\end{tabular}

15 Refer to Table 3.1 for $10 \mathrm{~kW}$ specifications, which are not varied in the study. 


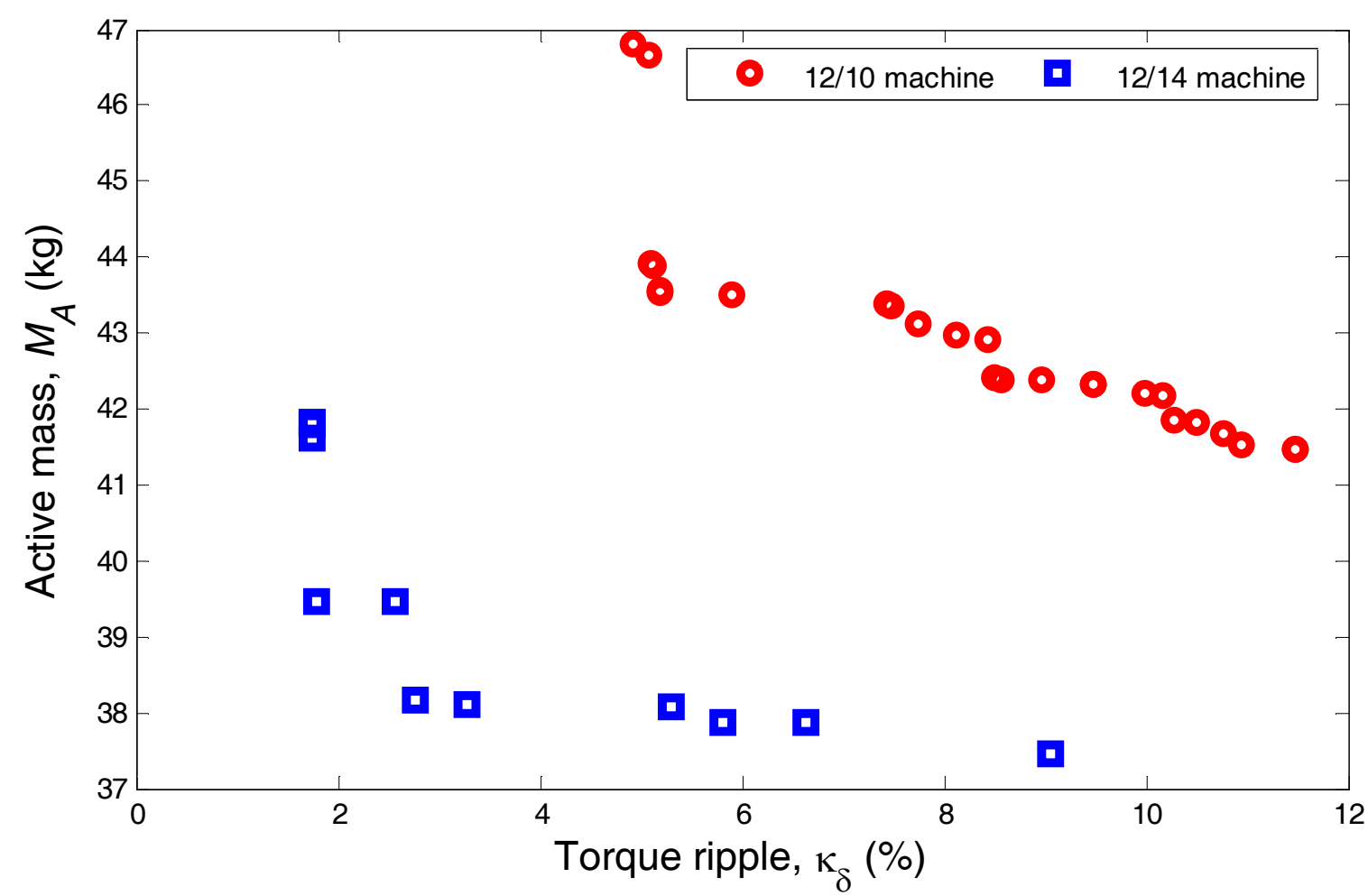

Fig. 3.2. Pareto optimal design candidates for $10 \mathrm{~kW}$ PM-FSMs.

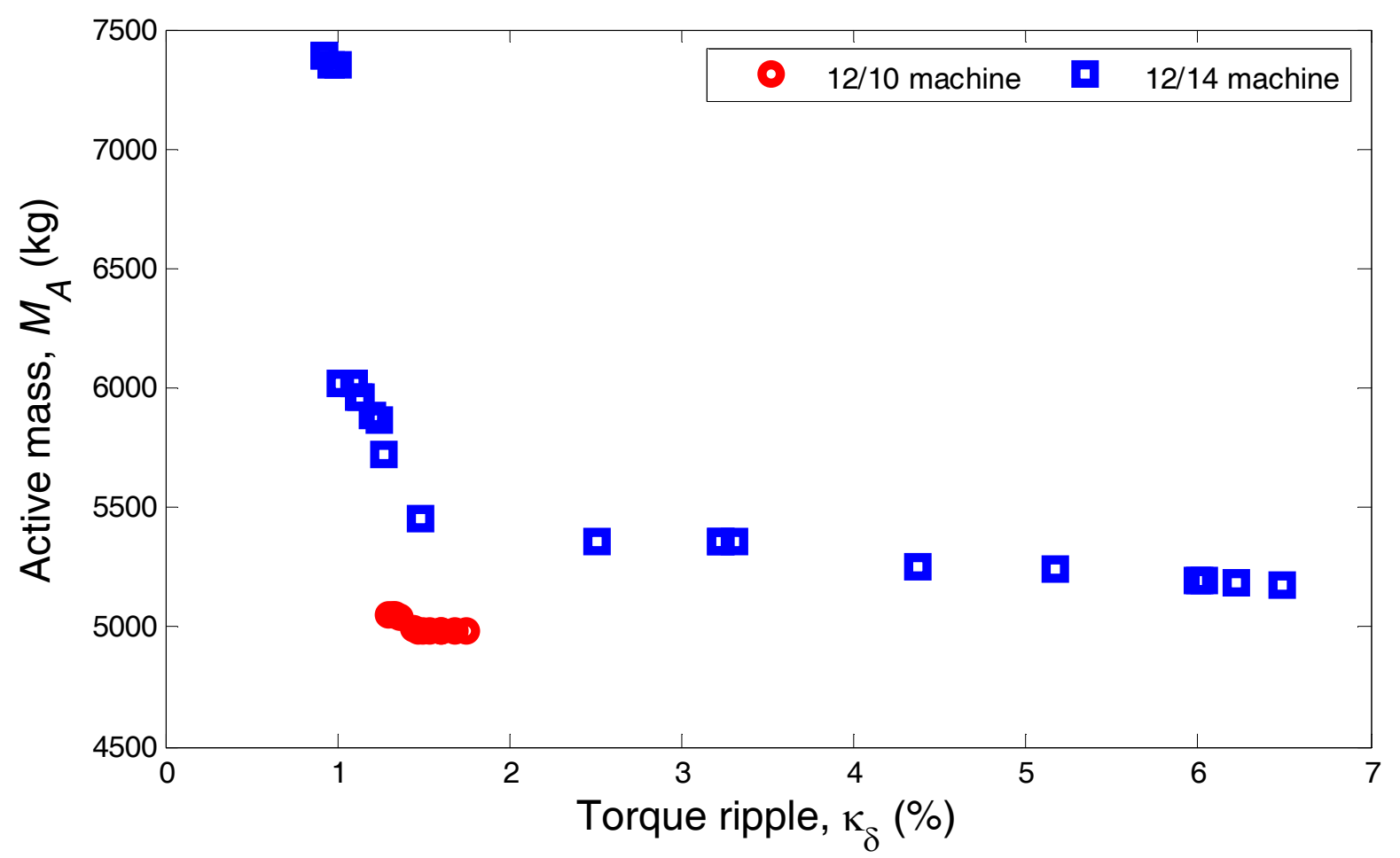

Fig. 3.3. Pareto optimal design candidates for 3 MW PM-FSMs.

To this end, five random optimal design candidates labelled ' $\mathrm{I}-\mathrm{V}$ ' and representing the 12/10 machine were chosen and their performance indices compared in per unit as shown in Fig. 3.4. The underlying factor in these selections is anchored on designs that are able to match the power specification at $3 \mathrm{MW}$. 


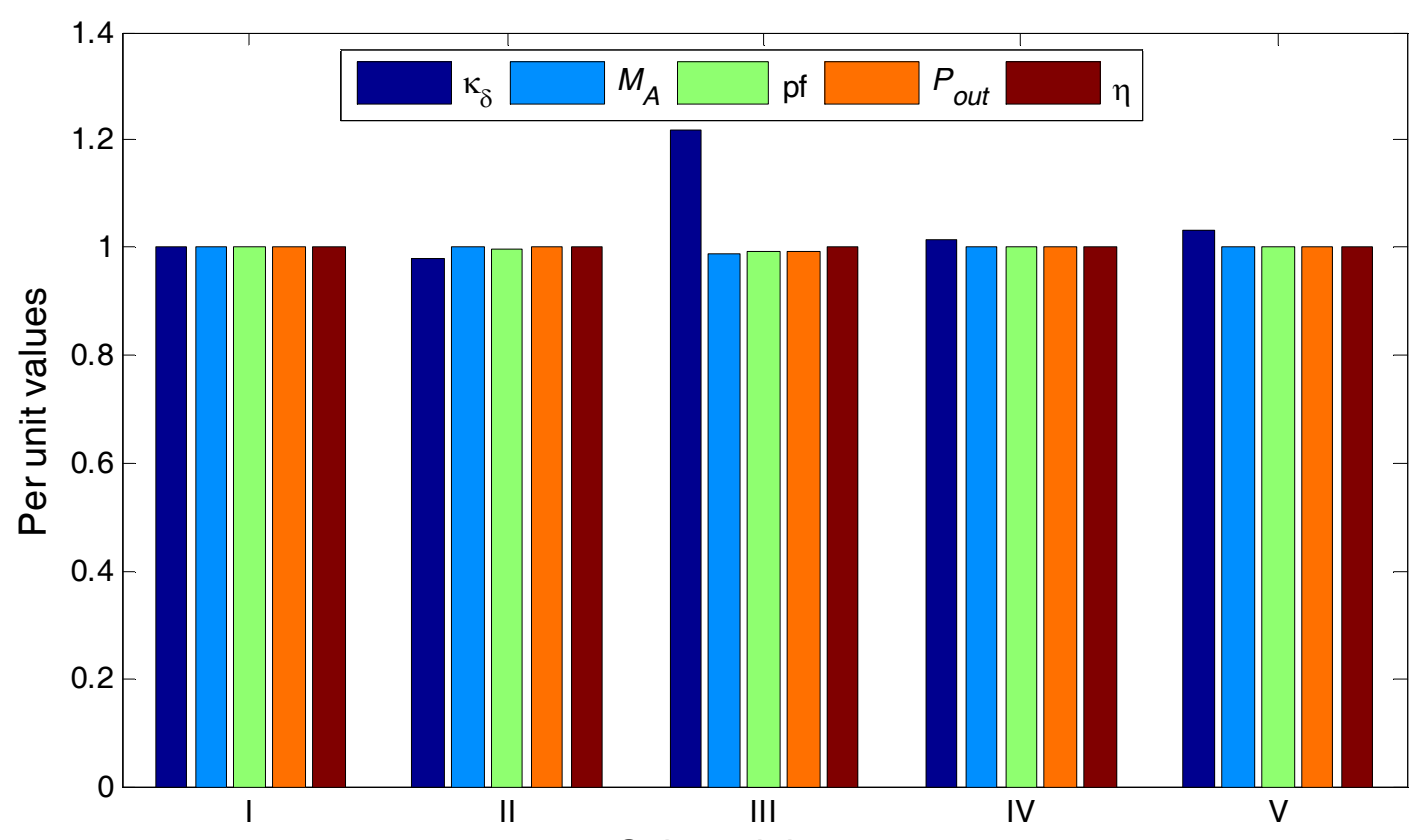

Selected designs

Fig. 3.4. Performance comparison of optimum design candidates of 12/10 $3 \mathrm{MW}$ machines at nomalised values and based on similar output power.

The same process, having the same selection criteria, is repeated for the $12 / 14$ machine leading to the optimal designs labelled as 'VI-X' and shown in Fig. 3.5. Lastly, another five optimal design candidates (XI-XV) shown in Fig. 3.6, are also chosen from the 12/14 optimal set. But this time, it is referenced to the same torque ripple value in design I. Also note that, all the performance indices compared in Figs. 3.4-3.6 are normalised with reference to the values indicated for design I.

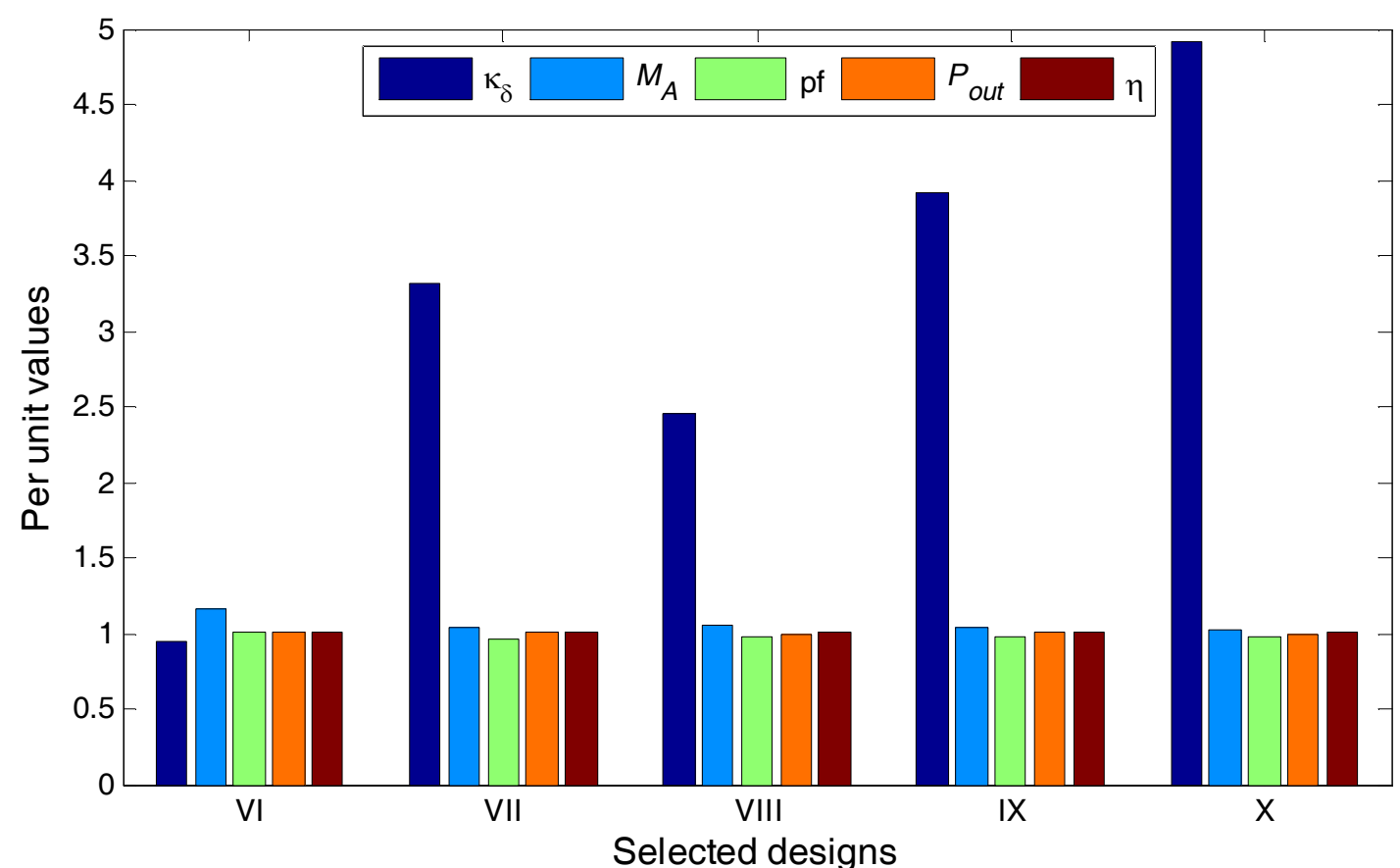

Fig. 3.5. Performance comparison of optimum design candidates of 12/14 $3 \mathrm{MW}$ machines at nomalised values and based on the same output power. 


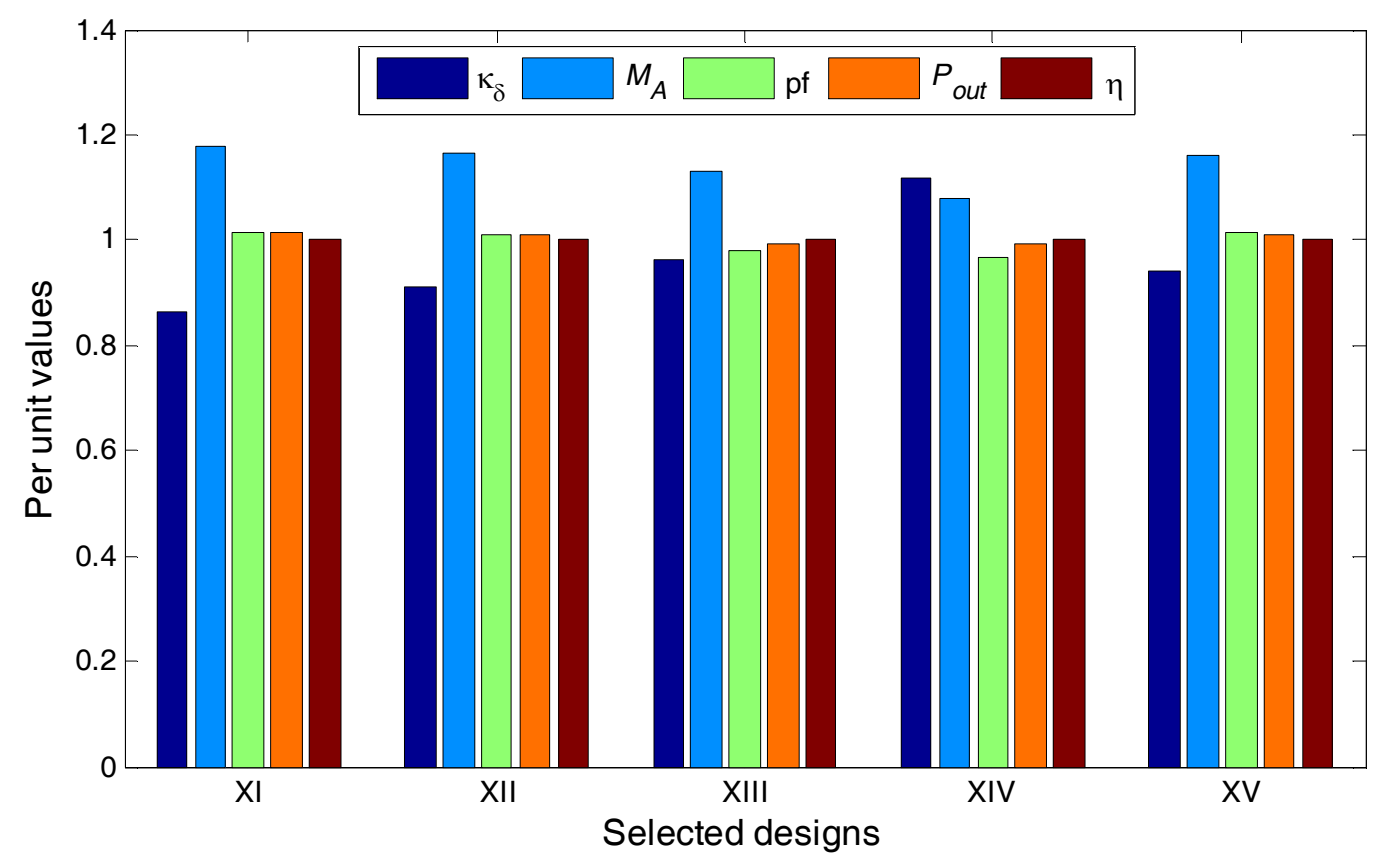

Fig. 3.6. Performance comparison of optimum design candidates of 12/14 $3 \mathrm{MW}$ machines at nomalised values, based on the torque ripple of design I.

In the first category (designs I-V), Fig. 3.4 shows that most of the optimal selections exhibit almost similar performance characteristics, because of a closely knitted optimal solution set as depicted in Fig. 3.3, while the second category (designs VI-X) in Fig. 3.5, which is dispersed by virtue of the optimal solutions indicated along the Pareto optimal front Fig. 3.3, portray dissimilar characteristics of the torque ripples. Meanwhile, Fig. 3.5 also reveals that for the same reference power as the $12 / 10$ machine, the optimum torque ripple values in the 12/14 machine are increased unusually, reaching up to five times in one instance.

To further grasp the behavior of the 12/14 machine at $3 \mathrm{MW}$ power levels, which deviates from that of its $10 \mathrm{~kW}$ designs, Fig. 3.6 shows that by sizing the optimum torque ripple of the $3 \mathrm{MW} \mathrm{12/14}$ machine to that of a 3 MW 12/10 machine, the active mass is increased to about $20 \%$. Then by comparing the optimal solutions of the 3 MW 12/14 machines, such as designs VI-X (obtained by baselining the output power to $3 \mathrm{MW}$ ) to those of designs XI-XV (obtained by baselining the torque ripple to that obtained in design 1), different patterns of certain key design variables are plotted as shown in Figs. 3.7-3.9.

Figs. 3.7-3.9 show that increase in PM volume, decrease in split ratio and decrease in current density are the main factors that constitute larger active mass in the 12/14 machines compared to the $12 / 10$ ones at $3 \mathrm{MW}$ power range. This is possible because, as indicated, the decrease in current density for the 12/14 machines is very significant, twice as less compared to that in the 12/10 machines. 


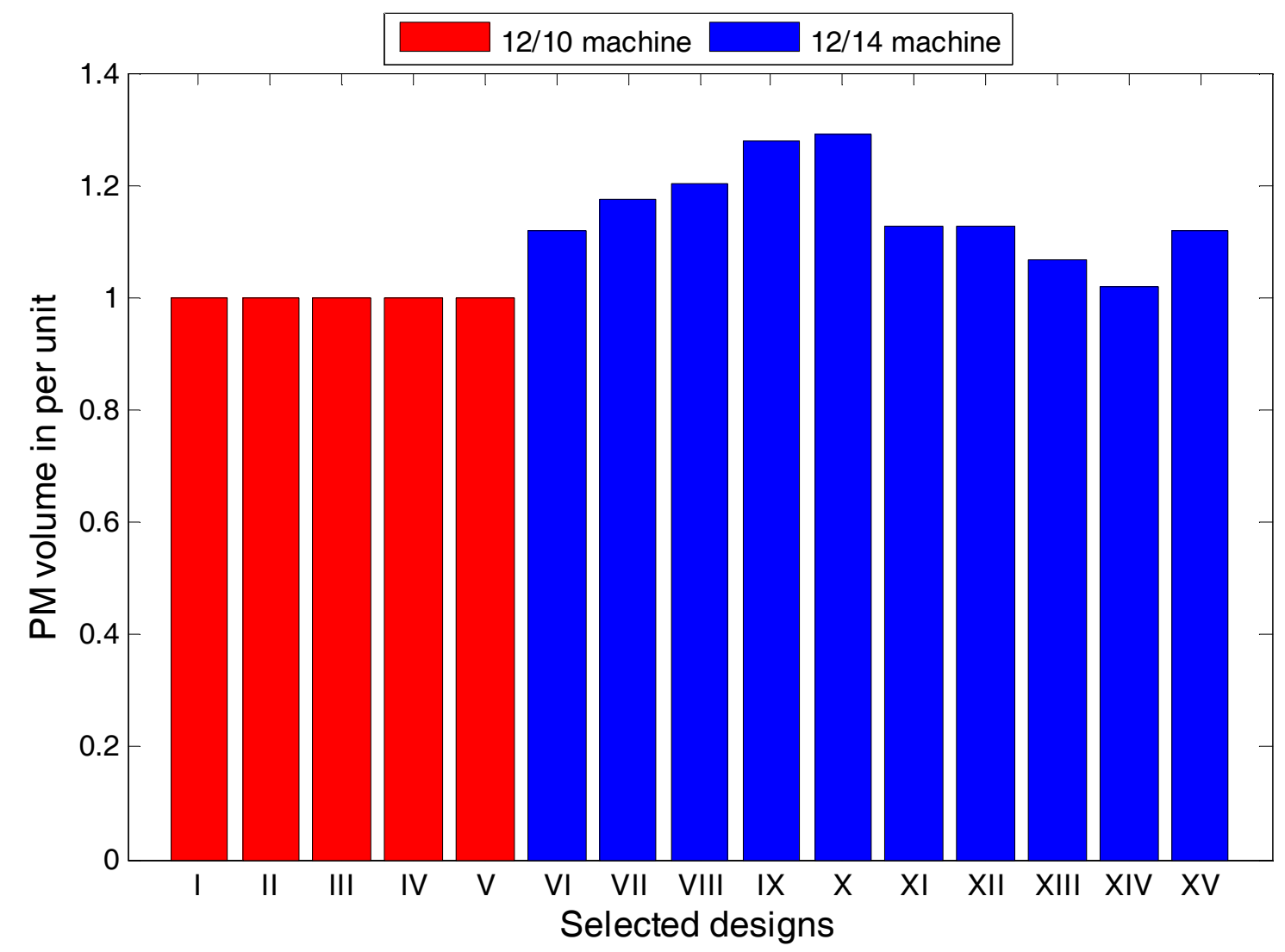

Fig. 3.7. Comparison of PM amount used in 3 MW optimum design candidates of $12 / 10$ and 12/14 machines ( 1 per unit volume is the value of PM volume used in design I).

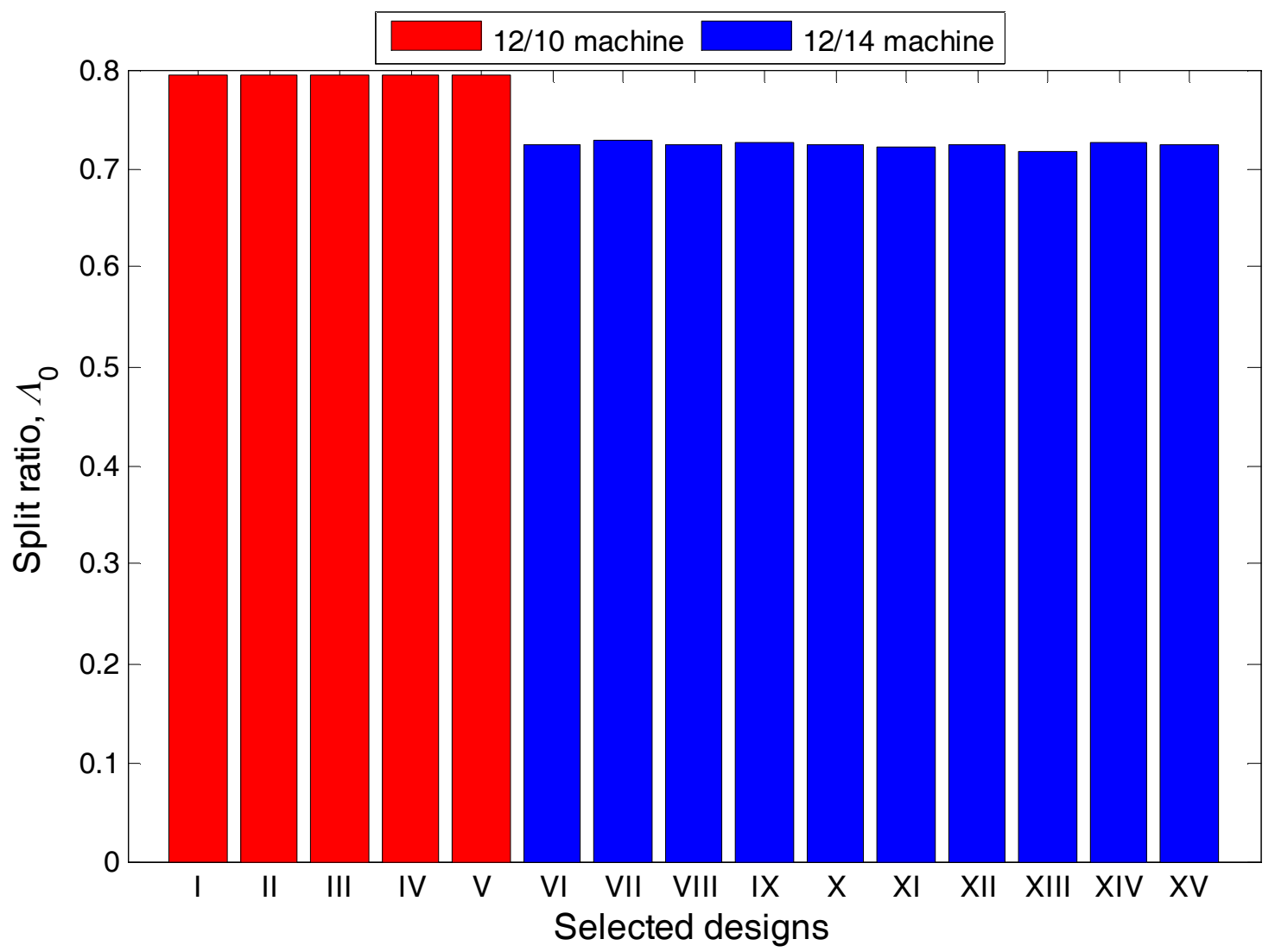

Fig. 3.8. Split ratios in selected $3 \mathrm{MW}$ optimum design candidates. 


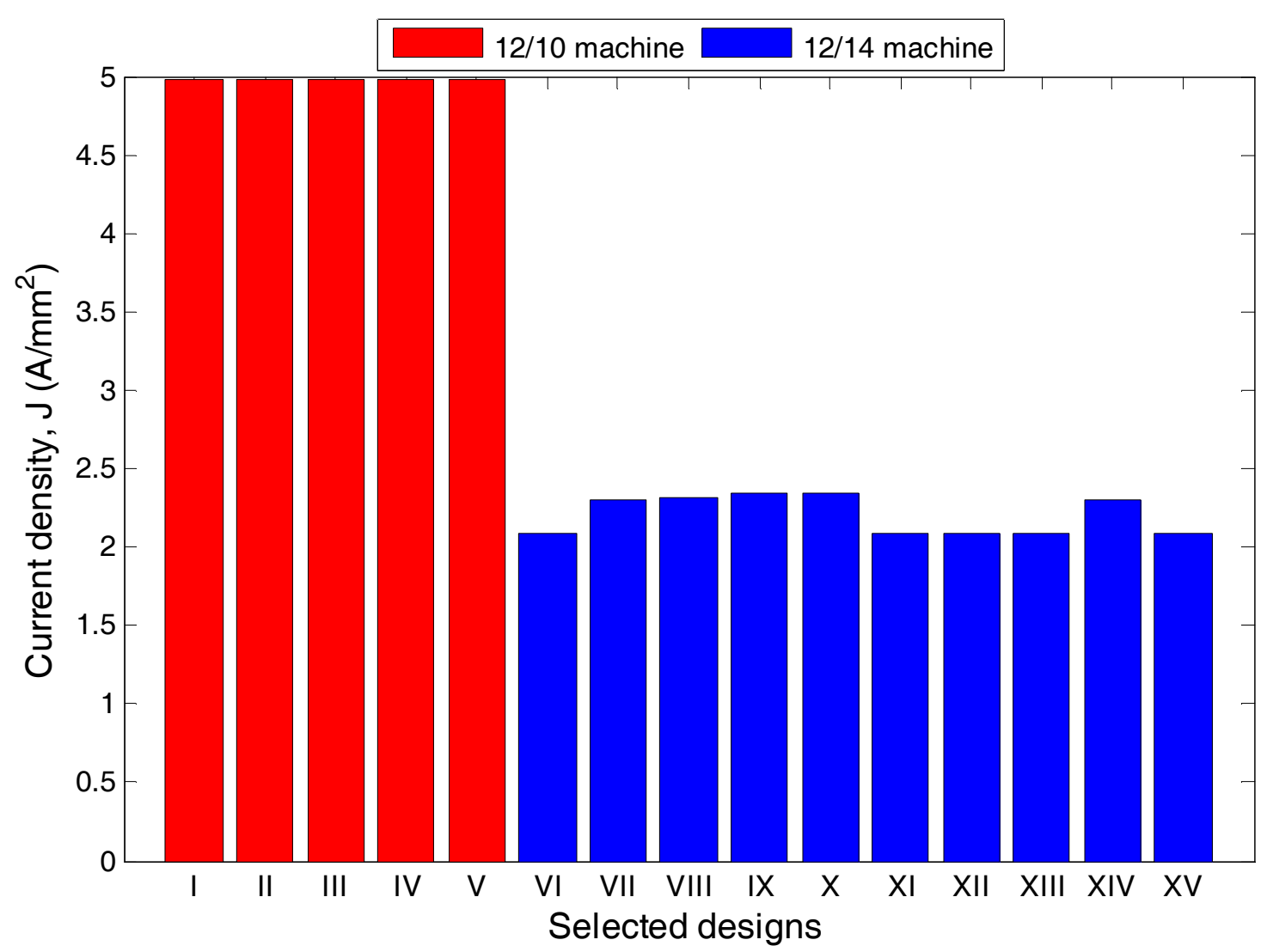

Fig. 3.9. Variation of current densities in selected $3 \mathrm{MW}$ optimum design candidates.

In addition, one can also observe a connection between the variations of the PM volume and the resulting torque ripple. For instance, torque ripple values in Figs. 3.4 and 3.6 are mostly low compared to those evaluated in Fig. 3.5, which when contrasted with the normalised PM volumes in Fig. 3.7, a pattern on the corresponding effects of their PM consumption is realised. Hence, a higher

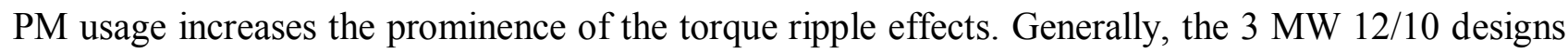
give better tradeoffs between the quantity of PM used and the generator active mass, as well as overall improvement in terms of the power density of the proposed wind power generators.

\subsection{Further Comparison and 3-D FEA Designs}

Two of the selected 3 MW optimal candidates, one from each machine as detailed in Table 3.4, areanalysed in 3-D transient FEA using ANSYS Maxwell ${ }^{\odot}$ to authenticate the time-stepped 2-D static FEA results obtained during the MDO process. In addition, Table 3.4 displays two other $10 \mathrm{~kW}$ optimal candidates randomly selected from the results earlier broadcasted in Fig. 3.2 (with both still adjudged on very similar torque ripple values). The selected $10 \mathrm{~kW}$ machines are used to further correlate with the performance of the $3 \mathrm{MW}$ machines, also shown. 
Table 3.4. Nomination and comparison of optimum candidates

\begin{tabular}{c|c|c|c|c}
\hline \multirow{2}{*}{ Parameters } & \multicolumn{2}{|c|}{ 3 MW machines } & \multicolumn{2}{c}{ 10 kW machines } \\
\cline { 2 - 5 } & 12/10: I & 12/14: XIII & $\mathbf{1 2} / \mathbf{1 0}$ & $\mathbf{1 2 / 1 4}$ \\
\hline \hline $\mathrm{P}_{\text {out }}(\mathrm{kW})$ & 3018.26 & 3044.43 & 10.02 & 10.12 \\
$\tau_{e}(\mathrm{Nm})$ & 78354.53 & 78908.75 & 261.03 & 262.24 \\
$\kappa_{\delta}(\%)$ & 1.34 & 1.28 & 5.73 & 5.88 \\
$P_{C u}(\mathrm{~kW})$ & 22528.93 & 6470.97 & 0.433 & 0.394 \\
$P_{\text {Core }}(\mathrm{kW})$ & 11806.32 & 21076.75 & 0.095 & 0.092 \\
$M_{A}(\mathrm{~kg})$ & 5049.74 & 5719.16 & 43.57 & 37.86 \\
$\eta(\%)$ & 98.87 & 99.10 & 94.99 & 95.40 \\
$\mathrm{PF}$ & 0.82 & 0.83 & 0.83 & 0.81 \\
$\Lambda_{0}$ & 0.79 & 0.72 & 0.56 & 0.60 \\
$\kappa_{L}$ & 0.56 & 0.57 & 0.81 & 0.78 \\
$\mathrm{Nm} / \mathrm{kg}$ & 15.51 & 13.40 & 5.99 & 6.93 \\
$\mathrm{kNm} / \mathrm{m}^{3}$ & 58.92 & 57.09 & 30.61 & 33.12 \\
$\mathrm{~J}\left(\mathrm{~A} / \mathrm{mm}^{2}\right)$ & 4.97 & 2.08 & 4.99 & 4.72 \\
\hline
\end{tabular}

\subsubsection{Performance Comparison based on Table 3.4}

It is observed that at $3 \mathrm{MW}$ output powers, larger split ratios evolve for the selected 12/10 machine, unlike its $12 / 14$ counterpart. At $10 \mathrm{~kW}$, both the split and aspect ratios are fairly comparable for the considered machines. No doubt, this immediately gives some insight on the behavior of the copper losses and current densities at the different power levels. Observe that the copper losses and current densities evolve to fairly the same values for the $10 \mathrm{~kW}$ machines but not for the $3 \mathrm{MW}$ machines, because of relatively smaller split ratio of the 12/14 machine at $3 \mathrm{MW}$. Perhaps, the potentially higher core losses of the 12/14 machine suppresses the current density, viz., the copper loss in order to satisfy the efficiency design limits, among other things.

It is also seen that the torque densities for the $3 \mathrm{MW} \mathrm{12/10} \mathrm{machines} \mathrm{are} \mathrm{more} \mathrm{than} \mathrm{two} \mathrm{times,}$ in fact almost triple, compared to their $10 \mathrm{~kW}$ machines, thanks to fixed rotor speed at both power levels. Whereas the torque density is higher for the $12 / 14$ compared to the $12 / 10$ at $10 \mathrm{~kW}$, the outcome, as earlier indicated, is reversed at $3 \mathrm{MW}$, no thanks to tradeoffs in the current densities and active masses. But considering the $10 \mathrm{~kW}$ machines and its fairly comparable design variables which evolved, it is safe to conclude that to emphasise the superior performance of the 12/14 machine compared to the 12/10 machine, in terms of torque density, their design space must be kept constant.

Amazingly, the efficiency of the 12/10 machine is lower for both the $10 \mathrm{~kW}$ and $3 \mathrm{MW}$ selected candidates when compared with their 12/14 counterparts. Such an outcome discredits the 1.4 times the fundamental frequency effect on the core loss which must always play out to the detriment of the 
12/14 machine, Anyuan et al: 2009 [106]. It is believed that this upturn has more to do with the optimal condition of certain design variables (e.g., split ratio, aspect ratio and current density) which evolved from the MDO process, rather than basing the selection criteria of the compared benchmarks only on their normalised torque ripple values. Besides, by visual inspection of the optimal design community, the researcher found that even if the selection criteria was rather on similar active mass, the $12 / 10$ machines were still able to operate at slightly lower efficiencies, generally. However, and perhaps, due to effects of a higher number of rotor teeth, which is implicated by a smaller split ratio, it has to be said that the $3 \mathrm{MW} \mathrm{12/14} \mathrm{machine} \mathrm{is} \mathrm{still} \mathrm{capable} \mathrm{of} \mathrm{a} \mathrm{lower} \mathrm{torque} \mathrm{density} \mathrm{compared} \mathrm{to}$ that of the $12 / 10$.

Meanwhile, by comparing the efficiency and power factor under varying load current as shown in Fig. 3.10, it is interesting to see that at $45 \%$ load current, both machines can operate close to unity power factor and almost at peak efficiency, at any of the power levels. More importantly is that the power factor specification (0.8) and efficiency specification (90\% at $10 \mathrm{~kW}$ and $97 \%$ at $3 \mathrm{MW}$ ) can be surpassed for both machines in the observed load range. The only exception is the efficiency of the 3 MW machines which dominates over just $80 \%$ of the load range, whereas the efficiency of the $12 / 10$ machine performs better when it is operating at less than $60 \%$ of the rated load current. Besides, the $3 \mathrm{MW}$ machines show better and more stable efficiency at higher loads. Overall, there is an excellent off-peak performance of the optimally designed PM-FSMs to suit the proposed wind generator and its associated drivetrain components in terms of efficiency and power factor.

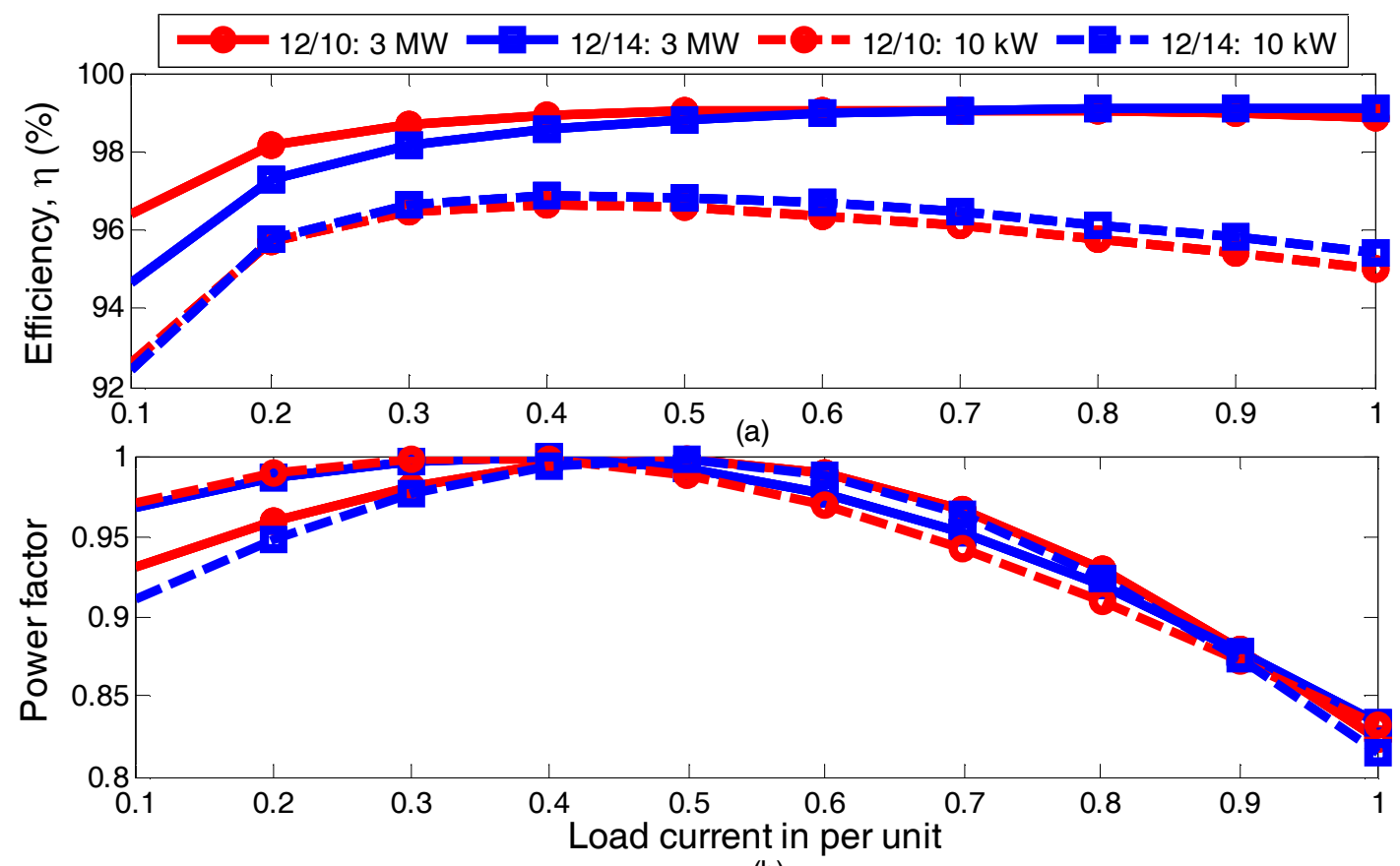

(b)

Fig. 3.10. Plots showing PM-FSMs load current profiles at optimum current angles against: (a) efficiency, and (b) power factor ( 1 per unit current is in terms of rated current in respective machine). 
Furthermore, it is also observed from Table 3.4 that the torque ripple values can be much lower in the $3 \mathrm{MW}$ machines compared to the $10 \mathrm{~kW}$ machines, a situation which was earlier visualised in the Pareto optimal plots in Figs. 3.2 and 3.3. It is indicated in Table 3.5 that it is due to relatively lower saliency ratios $(s)$ in the $3 \mathrm{MW}$ machines compared to their $10 \mathrm{~kW}$ counterparts-10\% and $15.2 \%$ for the $12 / 10$ and 12/14 machines, respectively. This is another important finding especially since PM-FSMs, due to its double saliency structure, are notorious for high torque ripple values. It then means that, for utility-scale wind turbines, such concerns might be less worrisome for the considered PM-FSM wind generator designs.

At this stage, it is difficult to conclude on the superiority of the $12 / 10$ over the $12 / 14$, or vice versa, because the performance of either machine appears to be more a function of the power level at which the design is undertaken. However, it is very clear that both machines possess more reputable performance characteristics at MW power, than at $\mathrm{kW}$. Thus, the superior performance of the $3 \mathrm{MW}$ machines, compared to the $10 \mathrm{~kW}$ machines, is an indication that the potentials of the proposed PMFSM machines for geared medium-speed wind power generators is, indeed, very high.

\subsubsection{3-D FEA Solutions}

To limit the computation time, quarter symmetry is employed in the 3-D FEA design approach undertaken. The flux density maps of the constructed models are as shown in Fig. 3.11. Also, a comparison of the average electromagnetic torque in 2-D and 3-D FEA, taken from no-load to full-load, are made for both machines as shown in Fig. 3.12. A good agreement is witnessed, but with slight deviation observed towards the rated load, in both machines. No doubt, this discrepancy at higher load profiles is due to worsening end-leakages not fully accounted for at the design stage of the 2-D FEA predictions. Note that, at higher load current, the saturation effects in the 3-D cores are increased. Thus, unlike in a 2-D FEA model without fringing along the axial direction, the effect which is very active in 3-D FEA is responsible for increasing the end-leakage flux. Essentially, an estimation of the discrepancy is given in the range of 4-8\% of these large machines, to assist in the accurate modelling of the end effects in the $2-\mathrm{D}$ FEA.

Table 3.5. Nomination and comparison of optimum candidates

\begin{tabular}{c|c|c|c|c}
\hline \multirow{2}{*}{ Parameters } & \multicolumn{2}{|c|}{ 3 MW machines } & \multicolumn{2}{c}{$\mathbf{1 0} \mathbf{k W}$ machines } \\
\cline { 2 - 5 } & $\mathbf{1 2 / 1 0 :} \mathbf{I}$ & $\mathbf{1 2} / \mathbf{1 4 :}$ XIII & $\mathbf{1 2} / \mathbf{1 0}$ & $\mathbf{1 2 / 1 4}$ \\
\hline \hline$\alpha($ deg.) & 64.061 & 40.607 & 70.795 & 30.332 \\
$I_{s(\mathrm{RMS})}(\mathrm{A})$ & 103.714 & 75.020 & 11.673 & 11.466 \\
$L_{d}(\mathrm{mH})$ & 564.017 & 590.309 & 114.894 & 93.759 \\
$L_{q}(\mathrm{mH})$ & 284.477 & 609.676 & 64.356 & 114.241 \\
$s$ & 0.504 & 1.032 & 0.560 & 1.218 \\
\hline
\end{tabular}




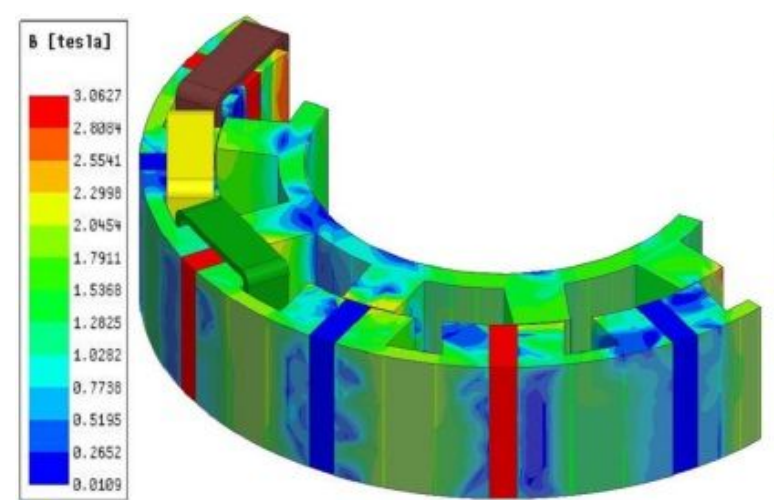

(a)

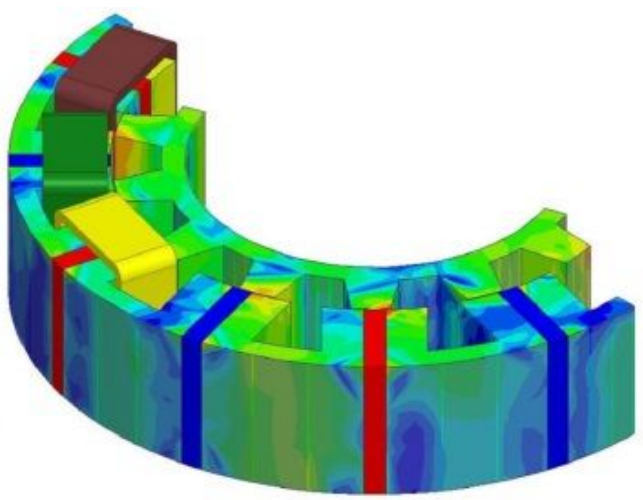

(b)

Fig. 3.11. Flux density maps of 3 MW PM-FSMs at rated conditions, analysed in 3-D transient FEA showing: (a) the 12/10 machine frozen at $5.21 \mathrm{~ms}$, and (b) the 12/14 machine frozen at $5.44 \mathrm{~ms}$.
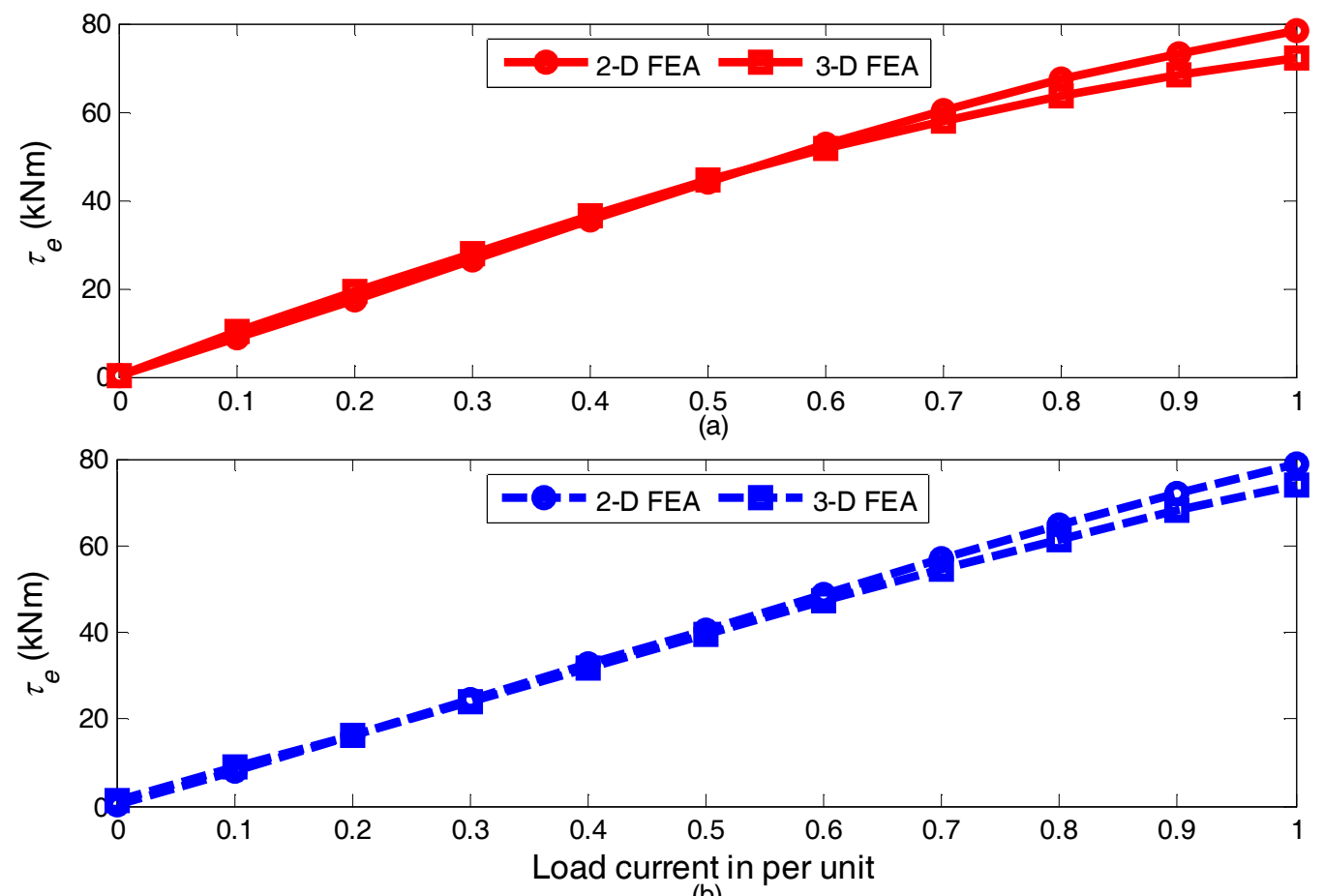

(b)

Fig. 3.12. Load current versus torque characteristics of 3 MW PM-FSMs displayed for: (a) 12/10 machine, and (b) 12/14 machine (1 per unit current is in terms of rated current in respective machine).

\subsection{Chapter Summary}

In this chapter, the design evaluation of optimum PM-FSMs at $10 \mathrm{~kW}$ and $3 \mathrm{MW}$ have been investigated for geared medium-speed wind generator drives. The design approach, machine topologies and MDO strategy have been presented. Based on 2-D static FEA, two PM-FSM topologies - the 12/10 and 12/14 machines - were optimised and compared at the prescribed power levels. The result shows that the torque ripple values in the $3 \mathrm{MW}$ machines can be much lower compared to the $10 \mathrm{~kW}$ machines due to disparities in their evaluated saliency ratios. Also, the torque densities are significantly higher in the $3 \mathrm{MW}$ machines than in the $10 \mathrm{~kW}$ machines, thanks to a fixed rotor speed at both pow- 
er levels. However, this is an indication of the high potentials of the proposed PM-FSMs for geared MS wind generator drives in large-scale wind turbine systems, which makes them eligible to be designed with the rare-earth-free excitation sources like ferrite PMs or wound-fields.

Moreover, it is observed that the superior optimum performance of the 12/14 machine compared to the $12 / 10$ machine at $10 \mathrm{~kW}$ power is reversed at $3 \mathrm{MW}$ power, due to significant decrease in the current densities of the 12/14 machines which increases both their torque ripple and active masses at the $3 \mathrm{MW}$ power range. A connection is also observed between the variations of the PM volume and the resulting torque ripple at $3 \mathrm{MW}$ such that the higher the PM usage, the more prominent the torque ripple effects.

Also, based on the MDO results, it is established that the fundamental frequency of the $12 / 14$ machine, which theoretically surpasses that of the $12 / 10$ machine by a factor of 1.4 , is not strategically disadvantageous to its efficiency, due to the optimal behaviour of certain key design parameters. This is generally observed in all the optimal solutions and for all power levels investigated, although it is has to be said that it results in the power density of the 12/14 machines, designed at $3 \mathrm{MW}$ power levels, to be slightly reduced. The observed tendency is in response to the design optimisation strategy implemented such that, for instance, the current density of the 3 MW 12/14 machine is less than half to that of the $3 \mathrm{MW} \mathrm{12/10} \mathrm{machine,} \mathrm{with} \mathrm{a} \mathrm{corresponding} \mathrm{increase} \mathrm{in} \mathrm{the} \mathrm{copper} \mathrm{loss} \mathrm{to} \mathrm{about}$ $350 \%$.

It has been equally found that the torque ripple values can be much lower in the $3 \mathrm{MW}$ machines compared to the $10 \mathrm{~kW}$ machines due to relative differences in their saliency ratiosevaluated at between $110 \%$ and $115 \%$ for the $10 \mathrm{~kW}$ machines. This finding is crucial because it further heralds the potential adoption of these machines for utility-scale wind generator applications, with less worry about the bad publicity on their high torque ripple effects.

As a final point, the two selections made from each category of the $3 \mathrm{MW}$ optimal candidates and compared between 2-D static and 3-D transient FEA results, with good confidence displayed. 


\section{ChApter Four}

\section{Performance Comparison and Investigation of Low- COST DESIGNS}

This chapter heralds the use of cheaper and easily accessible rare-earth-free materials which could serve as replacements for the more expensive and scarce rare-earth PMs. It is an attempt to study the potentials of using either ferrite PM or wound-field for the design and manufacturing of the proposed FSM wind generator. Again, the popular 12/10 machine is preferred for the present design optimisation and performance comparison which will be confronted in 2-D FEA, for power specifications at $10 \mathrm{~kW}$. The chapter also presents a separate investigation on cost savings per performance, highlighting also the demagnetisation risks, particularly when ferrite PM designs are compared at par with rare-earth PM designs. The chapter, which is a follow-up from the concluding remarks given in the preceding chapter, has also been published as conference papers in Akuru and Kamper (2016) [111] and Akuru and Kamper (2017) [112].

\subsection{Introduction}

The growth and prominence of wind energy development for electricity generation in the last two decades or so has been unprecedented, even as it is currently the most used among newer renewable energy technologies, REN21: 2017 [6]. This has prompted significant interest in the design and manufacturing of wind generators, which are major components in wind turbines. As a result, more and more researchers have focused on the use of non-conventional electrical machines for WPG, Potgieter and Kamper: 2012 [113], Wang et al: 2013 [114] and Boldea, Tutelea and Blaabjerg: 2014 [115].

The FSM is a typical non-conventional electrical machine that is currently metamorphosing. The traditional design of FSM incorporates PMs, Cheng et al: 2011 [44]. Consequently, commonplace in the literature is the use of $\mathrm{NdFeB}$ or SmCo rare-earth PMs in their designs, which is commercially unattractive due to the fact that FSMs are PM-intensive machines. Besides, rare-earth PMs are prone to price fluctuations and are relatively expensive as a result of limited geographic sourcing, amidst a rising market volume for ferrites as shown in Fig. 4.1.

Rare-earth-free PMs e.g., ferrites are expected to offer less expensive options in FSMs compared to rare-earth PMs, but they carry a greater risk of demagnetisation due to characteristically lower magnetic coercivity according to Fasolo, Alberti and Bianchi (2014) [69]. But despite this fact, McFarland, Jahns and El-Rafaie (2014) [55] have showed that the magnetic circuit in FSMs possess 
unique design characteristics that can conveniently forbear demagnetization during normal operation.

On the other hand, there are FSM topologies that are entirely free of PMs, viz., WF-FSMs. The main advantage of WF-FSMs is that their field sources are controllable, which is not achievable in PM-FSM configurations. Also, Zhou and Zhu (2014) [70] opine that WF-FSMs are beneficial for high temperature applications because they have zero risks of demagnetisation.

Yet, there are FSM options combining both PM and wound-field excitations such as HEFSMs, which also possess good field regulation capabilities. In Dupas et al (2014) [116] and Hoang et al (2015) [117], it is also shown that such topologies, while using rare-earth PMs, yield higher noload flux capability comparable to ferrite PM variants, but the latter is able to provide up to $80 \%$ more flux variation range under load. However, such flux weakening in HE-FSMs is mostly beneficial in automotive applications as demonstrated in Guang-Jin, Zhu and Jewell (2015) [118].

To this end, some researchers have recently attempted (yet with much emphasis on automotive applications) low-cost high-performance FSMs which use only wound-fields, ferrite PMs or reduced-rare-earth PM contents. For instance, a novel 9/5 WF-FSM was proposed and compared with other WF-FSM topologies in Zhou and Zhu (2014) [70], by considering the back-EMF, cogging torque and static torque. The popular 12/10 PM-FSM topology was also proposed, for the first time, in the same design structure as the PM-FSMs being considered in this dissertation. By using only wound-fields as in Tang et al (2012) [67], they were able to highlight the torque versus field weakening performance of such machine topologies. Moreover, in Sulaiman, Kosaka and Matsui (2012) [95], a different structured WF-FSM was designed and evaluated for maximum torque/power capabilities relevant to automotive applications.

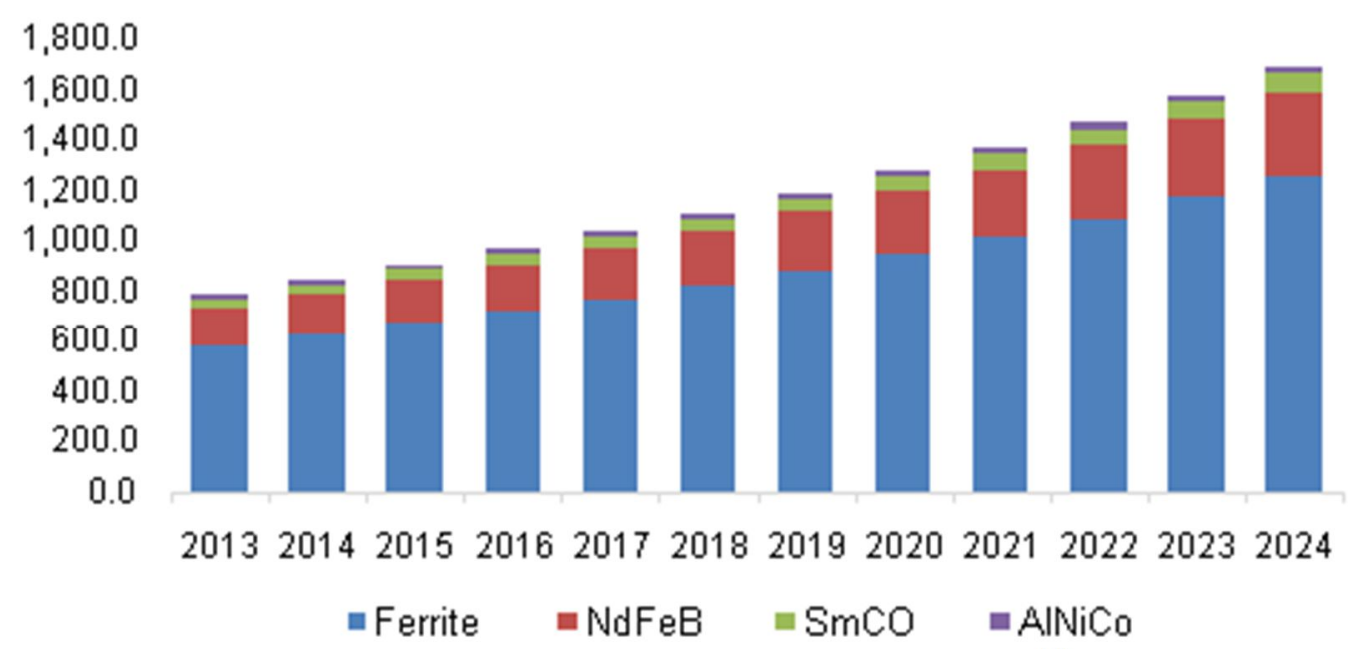

Fig. 4.1. Global market for permanent magnets from 2013-2024 ${ }^{16}$ (volume in kilotons). 
No doubt, the candidacy of FSMs for wind energy application is mounting as supported in an assessment study by Andrada and Martinez (2016) [120], however only a handful of researchers have attempted using non-rare-earth-PM field excitation sources as done in Wang et al (2013) [81] and Ojeda et al (2012) [37] for LS drivetrains. Geared MS drivetrains are important because they reduce the gear box size to 1 - or 2-stage from 3-stage or even more. Thus, unlike the traditional HS and LS systems which have been popular for wind generator designs, geared MS designs (say between 50 $600 \mathrm{r} / \mathrm{min}$ nominal speed) produce tradeoffs in terms of the size and cost of both the gear and generator units, Schmidt and Vath: 2012 [20].

In terms of optimisation for certain performance indices, Raminosoa et al (2015) [71] emphasized some design specifications such as mass, voltage, current and efficiency in pursuit of the potentials of FSMs for traction applications. By using two different grades of reduced-rare-earth designs and one wound-field variant, they showed that reducing or totally removing the rare-earth contents in rare-earth PM machines result in ample potentials of such designs for traction applications, depending on the design specifications. However, their study did not underscore the optimality of the design process.

In contrast, Tang et al (2013) [119] devised general correlations between various design parameters and machine performance considering the thermal condition, speed range and efficiency of rare-earth PM-FSMs in relation to WF-FSMs. Yet, they, as well, did not dwell on optimisation.

Similarly, the study by Fasolo, Alberti and Bianchi (2014) [69] only presented, without design optimisation, variants of ferrite and rare-earth PM-FSMs compared to conventional PM machines. They showed that the ferrite PM-FSM is an interesting solution in terms of lowest active material cost compared to the other machines considered, but with the worst demagnetisation behaviour. Nevertheless, the latter submission by Fasolo, Alberti and Bianchi (2014) [69] on the demagnetisation potentials of ferrite PM-FSM has been disputed by findings in another study conducted by McFarland, Jahns and El-Refaie: 2014 [55] where they found that, due to the arrangement of the PMs and the stator winding, their magnetic fields are constructively in sync to benefit the use of lower energyPMs like ferrites.

To this end, the optimal design and performance of parameters such as power factor and active mass, which are critical indices if a rare-earth-free FSM should be designed for wind generator drives, is yet to be studied. Hence, on one hand, this chapter documents a unique performance comparison on the power factor cum active mass relationship between ferrite PM-FSM and WF-FSM optimised for geared MS wind generator drives. On the other hand, it extends the analyses by focusing on the PM mass versus active mass of just the ferrite PM compared to rare-earth PM designs. All 
the considered machines are fashioned according to the popular 12/10 topology earlier pictured in Fig. 3.1(a) in section 3.2. As for the WF-FSM, it should be mentioned that although various structures do exist as highlighted in Zhou and Zhu (2014) [70] and Tang et al (2013) [119], but the preferred structure under consideration produces average torque comparable to PM-FSMs when based on the same electrical loading, Tang et al: 2012 [67].

For the rest of the chapter, the modelling process of FSMs as processed in the 2-D FEA package (SEMFEM) buoyed by its Python scripting functionalities, is rehashed. It is then followed by the first part of the study which is on the design optimisation strategy and performance evaluation of the candidate designs, after which the results are validated in 3-D FEA. Thereafter, a second design optimisation is undertaken to study the optimal performance of ferrite PM designs compared to rareearth PM designs, their cost tradeoffs and demagnetisation effects. The results of the second part of the study are also compared with transient FEA solutions in both 2-D and 3-D.

\subsection{Electromagnetic Modelling and Design Optimisation}

\subsubsection{Electromagnetic Modelling}

The electromagnetic modelling of FSM pursued in this section is not different from that already detailed in section 2.2 of this dissertation. The only difference is in the consideration of wound-field designs, which require the information on the formulation of the wound-field resistance, as well as a modification in the total copper loss estimation, presented as follows:

$$
\begin{aligned}
& R_{F}{ }^{17}=2 q_{F} N_{F}^{2} \rho_{C u} \frac{l_{s t}+l_{e F}}{A_{F}}, \\
& P_{C u}=3\left(I_{d}^{2}+I_{q}^{2}\right) R_{S}+I_{F}^{2} R_{F},
\end{aligned}
$$

where, $q_{F}$ is the number of wound-fields in series connection, $N_{F}$ is the turns number per coil for the field windings, $\rho_{C u}$ is the resistivity of copper wire at room temperature, $l_{s t}$ is the axial length of the lamination core, $l_{e F}$ is the end-winding length on one side of the field coil (refer later to Chapter 5, subsection 5.3.2 for full details), $A_{F}$ is the area of the field wire and $I_{F}$ is the field current. Other performance parameters retain their regular meaning.

\subsubsection{Design Optimisation Process, Results and Performance Comparison}

\subsubsection{Design Optimisation Process}

The design optimisation pursued here is still FEA-based, administered using the workflow illustrated in Fig. 1.13 in section 1.5. To give more insight, the optimisation is performed by coupling of the 2

17 It should be noted, for the benefit of the reader, that the end-winding length $\left(l_{e F}\right)$ used in the calculation of the total wound-field resistance of the WF-FSM topology as selected in this dissertation is fully described much later using Fig. 5.1 in Chapter 5. 
D static FEA package (SEMFEM) to the optimisation suite (VisualDOC). The VisualDOC optimiser is responsible for administering the non-gradient NSGA-II algorithm (refer to sections 2.3 and 3.3). The parameters adopted for the NSGA-II algorithm is summarised as shown in Table 4.1, while a general insight on their functionalities is discussed already in Deb et al (2002) [107].

The optimisation problems are propounded as constrained multi-objection design problems, with three objective parameters - minimum PM mass $\left(M_{P M}\right)$ for ferrite PM-FSM or wound-field mass $\left(M_{F}\right)$ for WF-FSM, minimum generator active mass $\left(M_{A}\right)$ and minimum torque ripple $\left(\kappa_{\delta}\right)$. The constraint parameters are the power factor $(\mathrm{PF})$, electromagnetic torque $\left(\tau_{e}\right)$ and efficiency $(\eta)$.

The various design variables as decided for the MDO are displayed much later in Table 4.2, with two benchmarks compared. But as indicated, the total number of design variables is 12 for the ferrite PM-FSM and 13 for the WF-FSM. Meanwhile, the airgap length is kept constant at $0.7 \mathrm{~mm}$ while the slot fill factors (of both wound field and phase coils) are also maintained at constant values of 0.45 for both the ferrite PM-FSM and WF-FSM.

The three objective parameters selected is due to the fact that the proposed study is undertaken to establish their design relevance for geared MS wind generator drives based on the following:

- It is known that FSMs have a propensity for high ripple effects due to their double salient structures, which in most cases are products of vibrations. Thus, to minimise the torque ripple is an attempt to manage this problem.

- Minimising the generator active or in particular the mass of the field excitation source is meant to improve the power density of the wind generator, while maintaining a reasonable cost of the main generator components.

The optimisation problem is defined as follows:

Minimise $\quad F(\bar{x})=\left[\begin{array}{lll}M_{P M / F} & M_{A} & \kappa_{\delta}\end{array}\right]^{T}$,

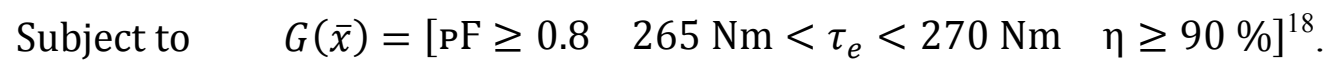

Table 4.1. NSGA-II parameter settings

\begin{tabular}{c|c|c}
\hline \multirow{2}{*}{ Parameter } & \multicolumn{2}{|c}{ Designs } \\
\cline { 2 - 3 } & ferrite PM-FSM & WF-FSM \\
\hline \hline Mutation probability, $P_{m}$ & 0.083 & 0.071 \\
Crossover probability, $P_{c}$ & 0.9 & 0.9 \\
Mutation distribution index, $\eta_{\mathrm{m}}$ & 10 & 10 \\
Crossover distribution index, $\eta_{\mathrm{c}}$ & 15 & 15 \\
Populations & 30 & 30 \\
Iterations & 100 & 100 \\
\hline
\end{tabular}

18 Note that, the electromagnetic torque constraint is meant to restrict the output power specification to within $10 \mathrm{~kW}$. In addition, to escalate the proposed geared MS drivetrains, the shaft speed is set to $360 \mathrm{r} / \mathrm{min}$. 


\subsubsection{Optimisation Results and Performance Comparison}

The resulting Pareto distributions are plotted as shown in Figs. 4.2 and 4.3. The objectives of the ferrite PM-FSM and WF-FSM, viz., $M_{P M / F}, M_{A}$, and $\kappa_{\delta}$, have been plotted based on a $\left(\begin{array}{l}3 \\ 1\end{array}\right)$ pairwise interactions. In most of the cases, it is observed that the convergence to the true Pareto optimal front is not explicit. For example, only the interaction between the PM mass and torque ripple clearly converged to the true Pareto optimal front for the ferrite PM-FSM as shown in Fig. 4.2(b).

In Fig. 4.2, we see the ability of the NSGA-II algorithm in producing diverse solutions for the WF-FSM design, while attempting to converge to the true Pareto optimal front. Thus, only the first solution set of the interactions between active mass and field coil mass, as well as active mass and torque ripple closely mimic the true Pareto optimal fronts.

No surprise, achieving the true Pareto optimal front is mostly influenced by the maximum number of generations evaluated, which in the cases considered is only 100 . With the population size set at 30, it results in a total number of 3000 design evaluations, which lasts for an average total optimisation time of 57.5 hours at $42.6 \mathrm{~s}$ per FEA solution using an i7-4770 CPU workstation. That notwithstanding, flares of the optimum feasible solutions have been produced, whereby some important inferences are made as follows:
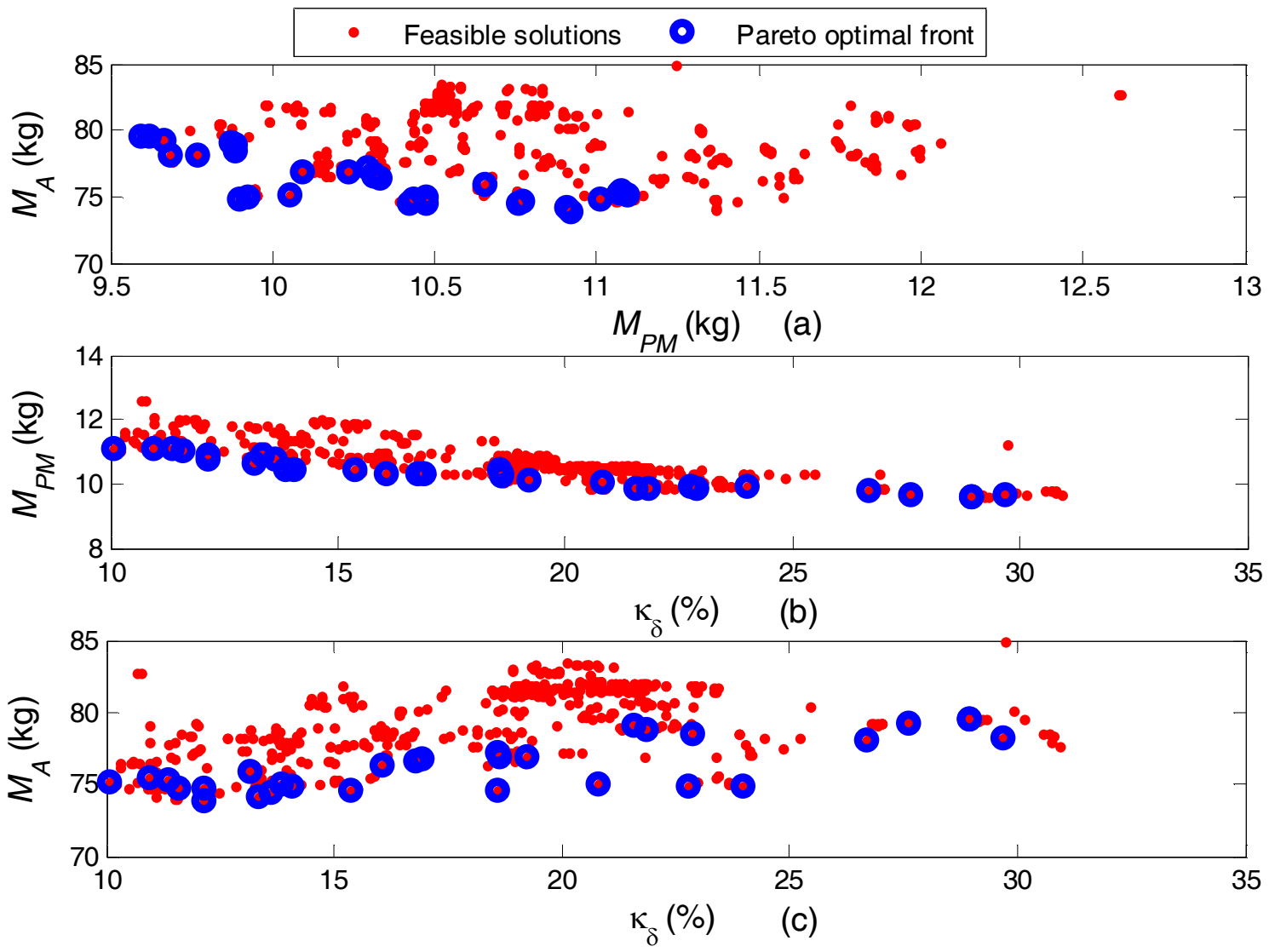

Fig. 4.2. Pareto optimal fronts for ferrite PM-FSM showing plots of: (a) $M_{A}$ against $M_{P M}$, (b) $M_{P M}$ against $k_{\delta}$, and (c) $M_{A}$ against $k_{\delta}$. 

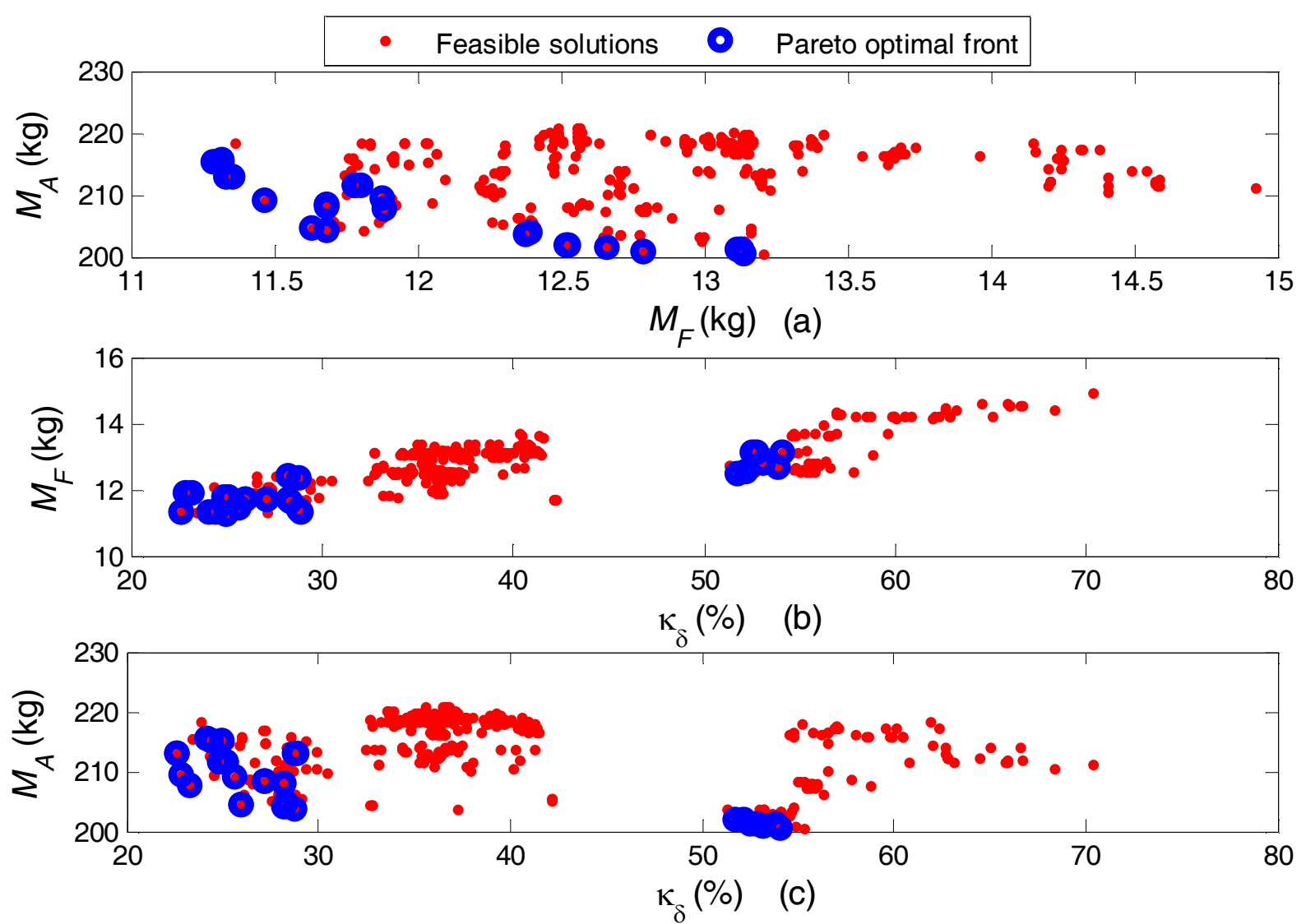

Fig. 4.3. Pareto optimal fronts for the WF-FSM showing plots of: (a) $M_{A}$ against $M_{F}$, (b) $M_{F}$ against $k_{\delta}$, and (c) $M_{A}$ against $k_{\delta}$.

- For the prescribed design requirements, the active mass of the ferrite PM-FSMs are significantly minimised compared to the WF-FSMs as observed in Figs. 4.2(a) and 4.3(a), respectively.

- A slight increase in the mass of the ferrite PMs lead to a drastic drop in the torque ripple values (refer to Fig. 4.2(b)), but the same cannot be said of the field coils which are observed in divergent solution sets in Fig. 4.3(b). Thus, reduced field coil masses result in decrease in torque ripple for the WF-FSM. Also, it is observed that based on comparable mass of the different field sources, a minimum torque ripple can be achieved in the ferrite PM-FSM which is less than half of that attainable in the WF-FSM.

- By observing the posture in Fig. 4.3(c), it is very clear that the active mass influences the torque ripple values in the WF-FSM. It also appears that the larger the generator active mass becomes, the smaller the torque ripple. For this to be the case, it is evident that the three objective functions and three constraints applied to the WF-FSM MDO problem appears to have over-penalised the problem as implied in Zhang, Ionel and Demerdash (2016) [89].

Hence, in order to explain the differing behaviour of the ferrite PM-FSM and WF-FSM towards satisfying the MDO objective and constraint requirements and based on the non-violated op- 
timal design candidates, a 3-D scatter plot which compares the aspect and split ratios ${ }^{19}$ is produced as shown in Fig. 4.4. As expected for PM machines, the aspect ratios of the ferrite PM-FSMs are significantly higher compared to the WF-FSM. On the other hand, the WF-FSM designs are predisposed to larger split ratios due to the presence of their field coils which also compounds the copper losses, Zhu et al: 2008 [62]. Thus, as revealed in Tang et al (2013) [119], there is generally a tradeoff correlated between the axial and radial dimensions in both PM-FSMs and WF-FSMs with respect to the specified design requirements.

To further elucidate on the findings, two optimal candidates, one each from the ferrite PMFSM and WF-FSM, are nominated for additional evaluations. The characteristics expressing the key design and performance parameters of both benchmarks are expressed in Table 4.2 from which it is very clear that the performance of the ferrite PM-FSM outclasses that of the WF-FSM in terms of efficiency, torque ripple, and torque density.

Also, the saliency ratios $\left(L_{q} / L_{d}\right)$ of both designs are compared and are found to be very much similar in response to the power factor at 0.8 , notwithstanding the fact that the $L_{d}$ and $L_{q}$ values are higher for the WF-FSM design. Thus, for the size and cost limitations of the SSCs, it goes to show that the power factor of the analysed machines are influenced by their saliency ratios. As noted in Sulaiman, Kosaka and Matsui (2012) [95], the sharp difference observed in the armature current densities in Table 4.2 is due to the need to give a good balance between both MMFs of the DC and AC windings. In that regard, the field and armature current densities of the WF-FSM design must be balanced in such a way that it satisfies the operating point of certain design requirements such as efficiency, torque and power factor quality, which apparently depends on the current commands.

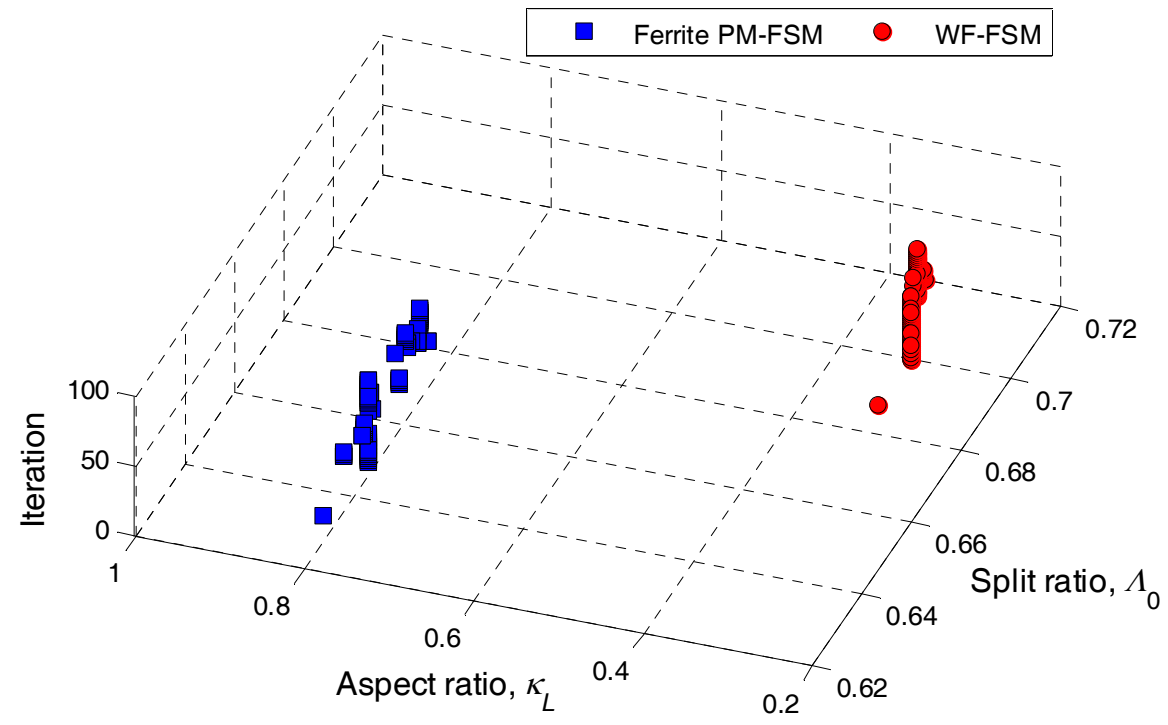

Fig. 4.4. Contrast between optimal aspect and split ratios.

\footnotetext{
19 Note that, the aspect and split ratios are defined in (A1.5) in Appendix A1.2.
} 
Table 4.2. Design and performance characteristics of $10 \mathrm{~kW}$ machines

\begin{tabular}{|c|c|c|c|}
\hline \multirow{2}{*}{ Parameter } & \multirow{2}{*}{ Symbol } & \multicolumn{2}{|c|}{ Value } \\
\hline & & Ferrite PM-FSM & WF-FSM \\
\hline Current angle (deg.) & $\alpha$ & 89.98 & 77.86 \\
\hline Armature current density $\left(\mathrm{A} / \mathrm{mm}^{2}\right)$ & $\mathrm{J}$ & 4.96 & 1.19 \\
\hline Stator outer diameter (mm) & $D_{\text {out }}$ & 339.90 & 634.17 \\
\hline Stator inner diameter (mm) & $D_{\text {in }}$ & 223.04 & 446.72 \\
\hline Shaft diameter (mm) & $D_{s h}$ & 99.724 & 248.46 \\
\hline Stack length (mm) & $l_{s t}$ & 172.28 & 136.42 \\
\hline PM or field core iron length (mm) & $b_{p m}$ or $b_{F}$ & 18.37 & 30.669 \\
\hline Rotor pole width (mm) & $b_{p r}$ & 25.25 & 69.87 \\
\hline Slot opening width (mm) & $b_{s l s}$ & 16.20 & 24.35 \\
\hline Stator yoke height (mm) & $h_{y s}$ & 22.88 & 34.96 \\
\hline Rotor yoke height (mm) & $h_{y r}$ & 12.05 & 38.90 \\
\hline Rotor tooth tapering factor & $t_{0}$ & 0.81 & 0.65 \\
\hline Field core iron width (mm) & $h_{F}$ & - & 28.01 \\
\hline Field current density $\left(\mathrm{A} / \mathrm{mm}^{2}\right)$ & $\mathrm{J}_{F}$ & - & 3.91 \\
\hline PM or field coil mass $(\mathrm{kg})$ & $M_{P M}$ or $M_{F}$ & 11.10 & 11.88 \\
\hline Active mass (kg) & $M_{A}$ & 75.20 & 209.50 \\
\hline Torque ripple $(\%)$ & $\kappa_{\delta}$ & 10.06 & 22.48 \\
\hline Power factor & $\mathrm{PF}$ & 0.80 & 0.80 \\
\hline Electromagnetic torque (Nm) & $\tau_{e}$ & 270.57 & 268.69 \\
\hline Machine efficiency $(\%)$ & $\eta$ & 94.95 & 91.06 \\
\hline Output power $(\mathrm{kW})$ & $\mathrm{P}_{\text {out }}$ & 10.32 & 10.38 \\
\hline Torque/active mass $(\mathrm{Nm} / \mathrm{kg})$ & - & 3.60 & 1.28 \\
\hline $\mathrm{d}-$ axis inductance $(\mathrm{mH})$ & $L_{d}$ & 46.26 & 74.61 \\
\hline $\mathrm{q}-$ axis inductance $(\mathrm{mH})$ & $L_{q}$ & 66.61 & 82.61 \\
\hline
\end{tabular}

Table 4.3. Cost quote of generator materials [69]

\begin{tabular}{c|c}
\hline Item & Cost (USD/kg) \\
\hline \hline NdFeB PM & 60 \\
Ferrite PM & 10 \\
Copper & 11.2 \\
Iron lamination & 2.2 \\
\hline
\end{tabular}

Based on the price schedule in Table 4.3, an estimated cost comparison of the components used in the design of both machines is processed as shown in Fig. 4.5. Again, the ferrite PM-FSM clearly presents a cheaper option due to its comparatively smaller active mass.

The 2-D FEA rated field distributions of the candidate designs are presented as shown in Fig. 4.6, with plots of their respective airgap flux densities shown in Fig. 4.7. Although the WF-FSM encountered a higher peak airgap flux density at $2.01 \mathrm{~T}$ compared to $1.83 \mathrm{~T}$ for the ferrite PM-FSM, the average flux density in the ferrite PM-FSM when evaluated along the reference airgap arc, is almost twice that of the WF-FSM, both estimated at $0.41 \mathrm{~T}$ and $0.28 \mathrm{~T}$, respectively. Besides Fig. 4.6 shows that, as common in PM machines, the leakage flux is more dominant in the ferrite PM-FSM than in WF-FSM. 


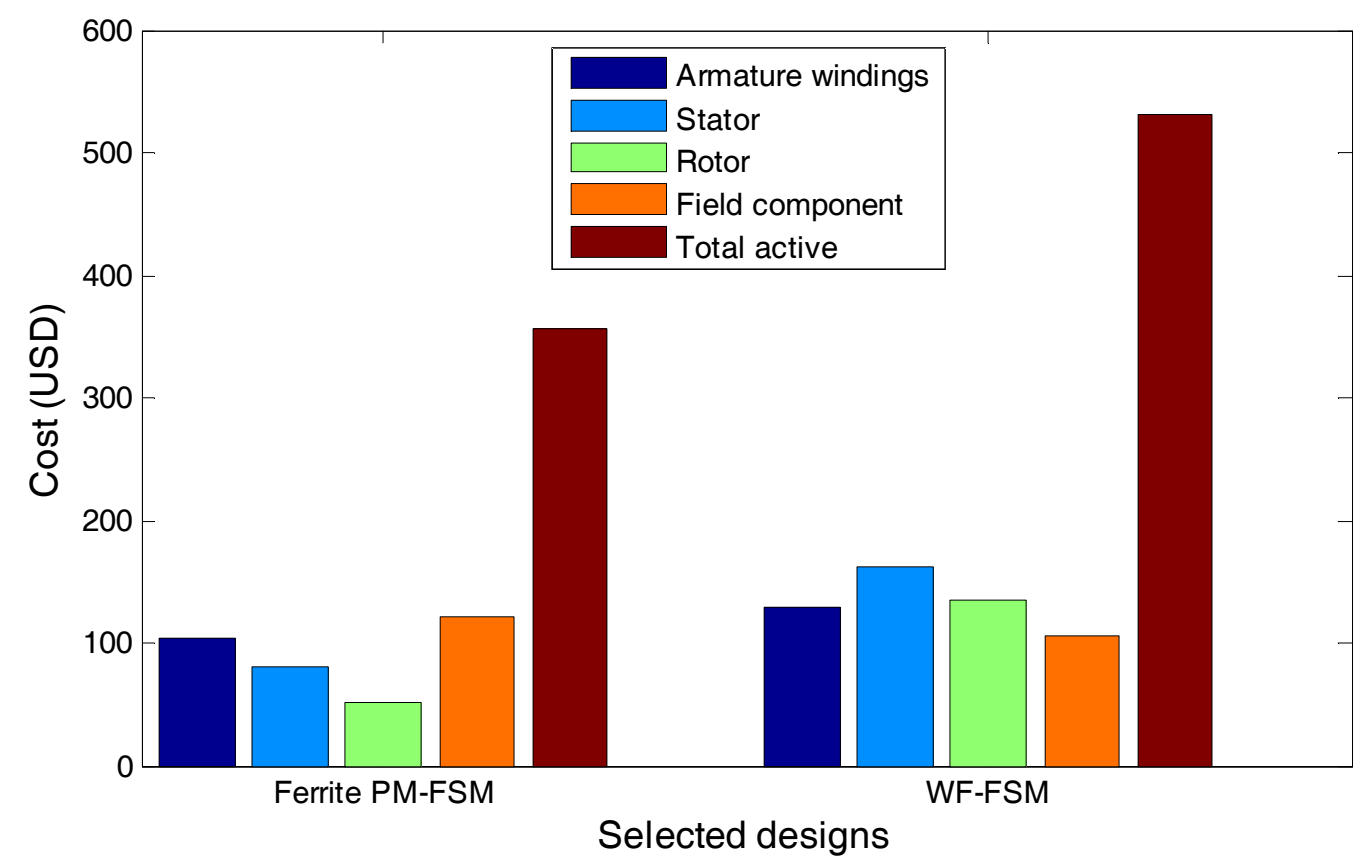

Fig. 4.5. Comparison of different component costs.

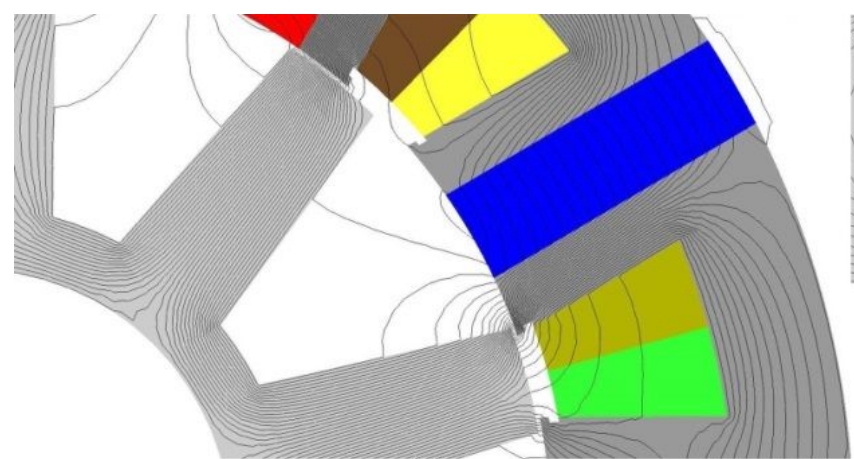

(a)

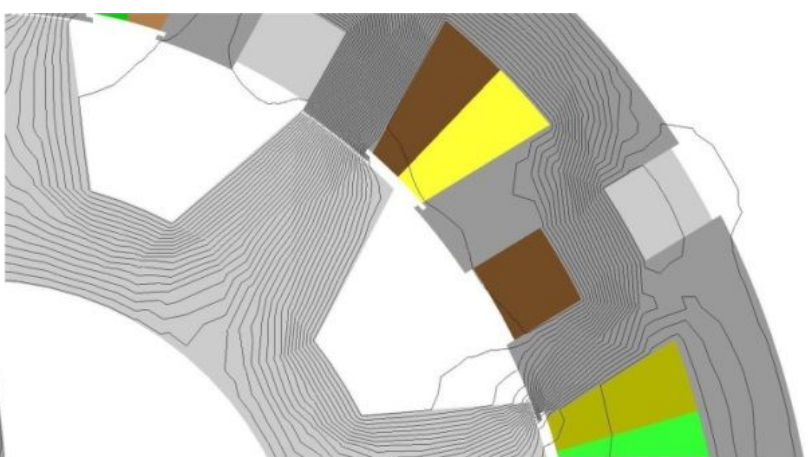

(b)

Fig. 4.6. Cut-out cross-sectional views of magnetic field distributions at rated conditions analysed in the benchmarked optimal designs, viz., (a) ferrite PM-FSM, and (b) WF-FSM.
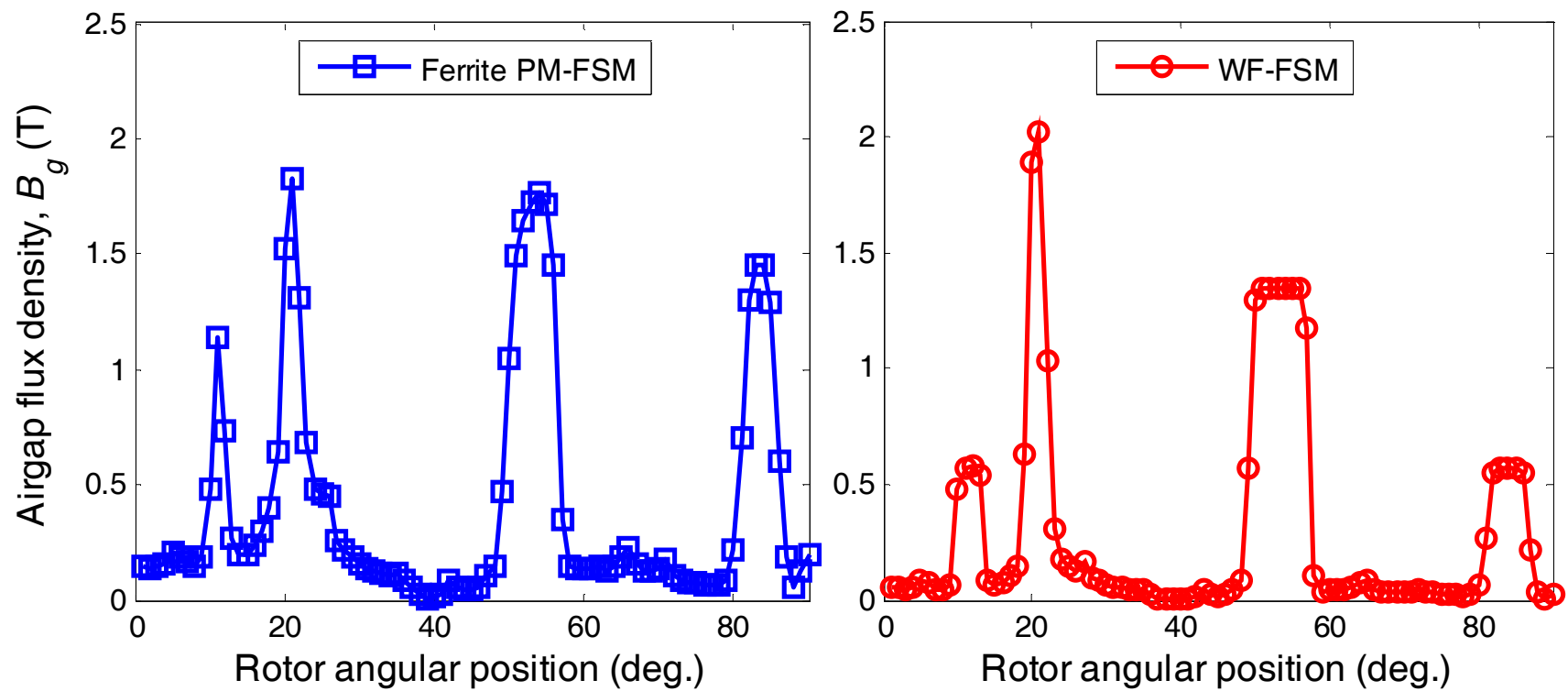

Fig. 4.7. Plots of airgap flux densities under rated conditions. 


\subsection{Investigation of Ferrite PM-FSMs versus Rare-Earth PM-FSMs}

Based on the preliminary results which portray the candidature of the $10 \mathrm{~kW}$ ferrite PM-FSM for the proposed wind generator drivetrain as superior compared to the $10 \mathrm{~kW} \mathrm{WF-FSM,} \mathrm{this} \mathrm{section} \mathrm{is} \mathrm{used}$ to provide additional investigation on the cost savings derivable when the ferrite PM-FSM is rather compared to its rare-earth PM-FSM counterpart, based on similar performance limits.

To this end, the investigation is instituted by undertaking a fresh design optimisation of both PM-FSM topologies based on the 2-D FEA process already escalated. Thereafter, the performance comparison of two benchmarks would be accomplished. Furthermore, demagnetisation studies would be executed on the selected benchmarks before finally proving the 2-D FEA results with 3-D FEA.

\subsubsection{Design Process and Optimisation}

At first, the 12/10 PM-FSM topology is once again designated for this analysis. The design process to be used is summarised in the flowchart given in Fig. 4.8. Both the ferrite and rare-earth PM-FSMs are subjected to the same optimisation problems based on the target specifications advertised in Table 4.4.

The design optimisation process involved the simultaneous evaluation of two objective functions, namely, PM mass $\left(M_{P M}\right)$ and total active mass $\left(M_{A}\right)$, with four design constraints, already indicated in Table 4.4, which ensure that the proposed wind generators achieve acceptable operating points. The design variables are composed in a transposed vector of design variables given as

$$
\bar{x}=\left[\begin{array}{lllllllllll}
\alpha & \mathrm{J} & l_{s t} & D_{i n} & D_{s h} & b_{P M} & b_{p r} & b_{s l s} & h_{y s} & h_{y r} & t_{0}
\end{array}\right]^{T},
$$

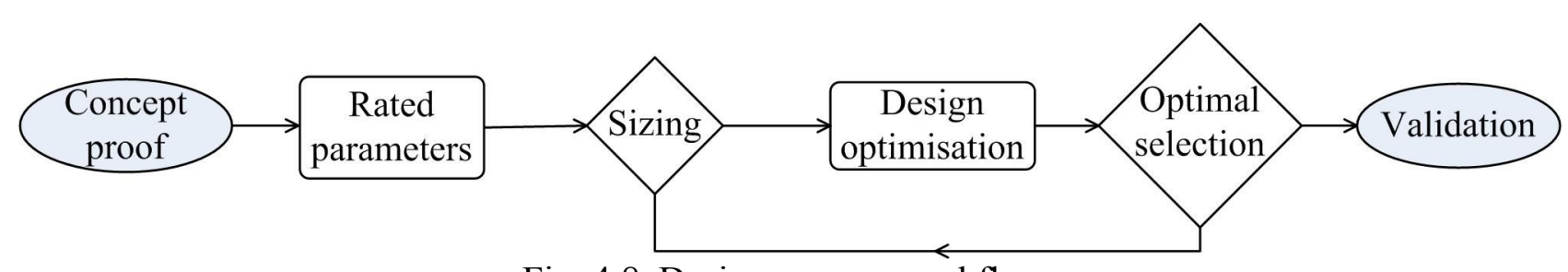

Fig. 4.8. Design process workflow.

Table 4.4. Design targets and parameter specifications

\begin{tabular}{c|c|c}
\hline \multirow{2}{*}{ Parameters } & \multicolumn{2}{|c}{ Designs } \\
\cline { 2 - 3 } & Rare-earth & Ferrite \\
\hline PM remanence, $B_{r}$ & $1.2 \mathrm{~T}$ & $0.4 \mathrm{~T}$ \\
Stator outer diameter, $D_{\text {out }}$ & $250 \mathrm{~mm}$ & $350 \mathrm{~mm}$ \\
Rated speed, $n_{s}$ & \multicolumn{2}{|c}{$360 \mathrm{r} / \mathrm{min}$} \\
Output power, $\mathrm{P}_{\text {out }}$ & \multicolumn{2}{|c}{$\geq 10 \mathrm{~kW}$} \\
Efficiency, $\eta$ & $\geq 90 \%$ \\
Power factor, $\mathrm{PF}$ & \multicolumn{2}{|c}{$\geq 0.8$} \\
Torque ripple, $\kappa_{\delta}$ & $\leq 10 \%$ \\
\hline
\end{tabular}


whereby all the variables have their usual meanings as defined in Table 4.2. Thus, the total number of decision variables as quoted in (4.5) is 11. The stator outer diameters were evaluated based on (A1.4) in Appendix A1.2 as indicated in Table 4.4, and kept constant for the duration of the design optimisation.

A total of 2000 function evaluations are compiled based on a population size of 20 for 100 generations. Key parameter settings such as the crossover probability, crossover distribution index and mutation distribution index are tuned to $0.95,10$ and 15 , respectively. Note that, the mutation probability is taken as the inverse of the number of design variables. As expected, the optimisation time used to complete a single run in a Core i7 CPU 16 GB RAM computer unit is 28.945 hours and 30.063 hours for the ferrite and rare-earth PM-FSM, respectively.

\subsubsection{Results}

The design optimisation results are displayed by the scatter plots in Fig. 4.9, which clearly shows a wide margin gulfing the colonies of the optimal set of the PM-FSM variants. At a closer look, it is observed that the ferrite PM-FSM designs consumes at least 1.5 times of the active mass and 2 times of the PM mass of that of the rare-earth PM-FSM designs.

Also, from the broadcasted results in Fig. 4.9, two sample designs, one from each PM-FSM variants, are benchmarked for further evaluation. The selected designs are highlighted as DI (for the rare-earth PM-FSM) and DII (for the ferrite PM-FSM) from the optimal design collections.

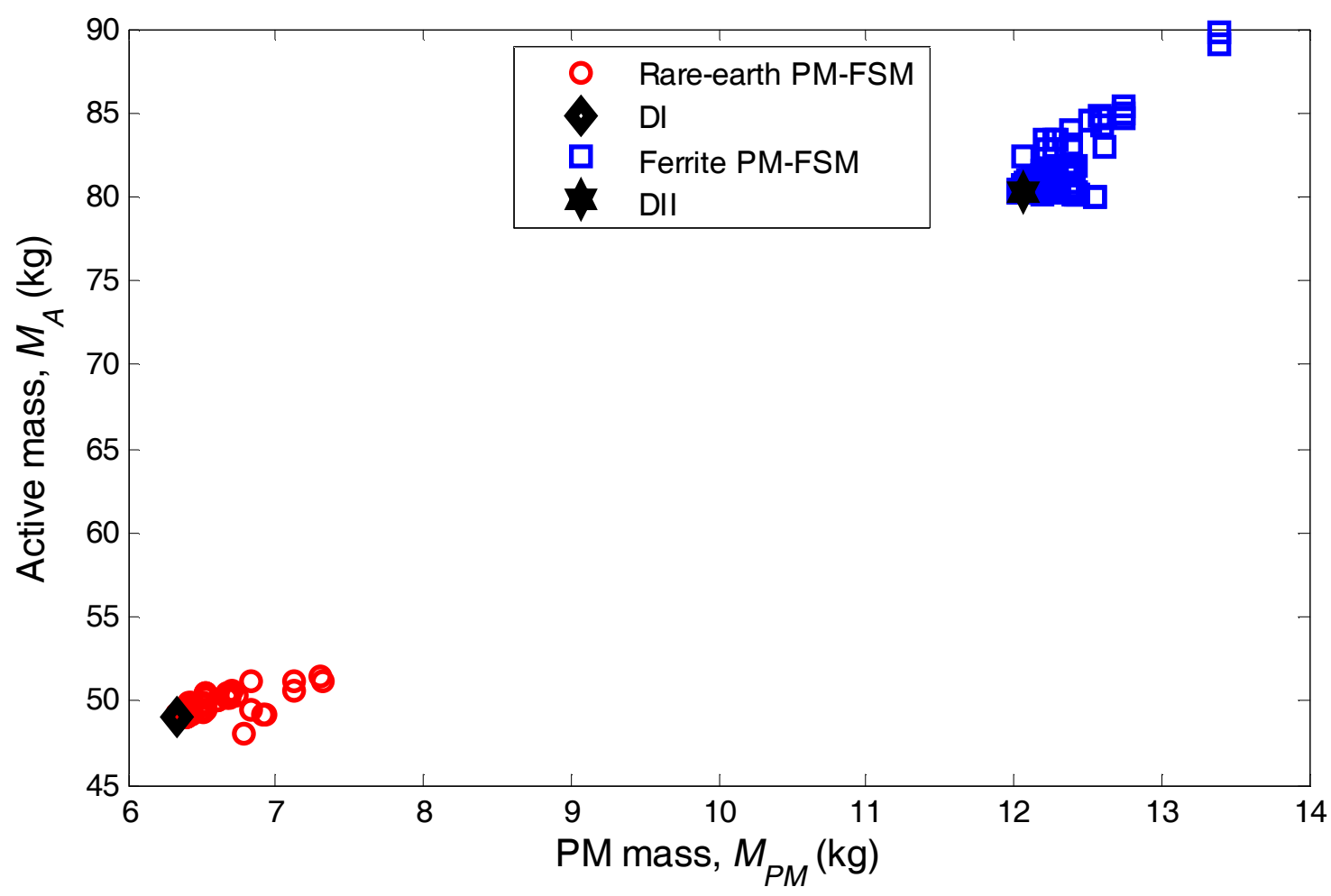

Fig. 4.9. Spread of the evolved optimal design candidates. 
Table 4.5 displays the optimum design parameters obtained for the preferred designs. It is observed that for the same design requirements, the tradeoffs between the optimal PM mass to active mass results in a narrower PM length and longer stack length for the rare-earth design, compared to the ferrite PM-FSM. In particular, the ratio of PM length to stack length is $4.2 \%$ in the former, compared to $11.3 \%$ in the latter. The outcome is because PM-FSMs, having flux focusing capabilities, are able to etch out thinner PM lengths when superior rare-earth PMs is used as against low-energy ferrite PMs.

As done in Fig. 4.4, the aspect $\left(\kappa_{L}\right)$ and split $\left(\Lambda_{0}\right)$ ratios are both compared for the optimal designs once again. The contrast is illustrated as shown in Fig. 4.10, which shows that the aspect ratio, more than the split ratio, is critical for the optimal performance of the rare-earth design. As for the lower quality ferrite PM, the split ratio is usually much more escalated in terms of boosting the corresponding airgap magnetic fields. Then, as indicated in Table 4.5 and later in Table 4.6, the discrepancy in current densities tends to influence the copper losses viz., the efficiency. With the theoretical slot filling factor of both designs kept constant at $45 \%$, it is observed that a higher current density value corresponds, more significantly, to higher copper loss and lower efficiency in the rare-earth PM-FSM compared to the ferrite PM-FSM.

Table 4.6 displays further comparison of both PM-FSM benchmarks. The torque density for the rare-earth is $63.2 \%$ more than the ferrite; thanks to its potentials to reduce the wind generator head mass. But from an economic standpoint, the total material cost of the ferrite design is only 65 $\%$ of the rare-earth design, based on quotations earlier documented in Table 4.3. Thus, in terms of the cost of materials, Fig. 4.11 clearly shows that the rare-earth PM-FSM is more expensive to manufacture, no thanks to the high cost of its PMs. Thus, the ferrite PM-FSM suffers lower torque/mass ratio but experiences higher torque/cost ratio compared to its rare-earth counterpart.

Table 4.5. Optimal design parameters

\begin{tabular}{c|c|c|c}
\hline$\overline{\boldsymbol{x}}$ & Parameters & Rare-earth (DI) & Ferrite (DII) \\
\hline$\alpha$ & Current angle (deg.) & 89.984 & 89.997 \\
$\mathrm{~J}$ & Phase current density (A/mm $\left.\mathrm{mm}^{2}\right)$ & 4.982 & 3.607 \\
$l_{s t}$ & Stack length $(\mathrm{mm})$ & 178.948 & 165.529 \\
$D_{i n}$ & Stator inner diameter $(\mathrm{mm})$ & 146.287 & 220.626 \\
$D_{s h}$ & Shaft diameter $(\mathrm{mm})$ & 80.029 & 119.879 \\
$b_{p m}$ & PM length $(\mathrm{mm})$ & 7.582 & 18.793 \\
$b_{p r}$ & Rotor pole width (mm) & 20.438 & 24.004 \\
$b_{s l s}$ & Slot opening width (mm) & 7.282 & 13.801 \\
$h_{y s}$ & Stator yoke height (mm) & 7.711 & 18.420 \\
$h_{y r}$ & Rotor yoke height (mm) & 13.784 & 18.750 \\
$t_{0}$ & Rotor teeth taper factor & 0.738 & 0.850 \\
\hline
\end{tabular}




\subsubsection{Demagnetisation effects}

A common characteristic of PMs is their demagnetisation susceptibility at high temperatures or as a result of excessive current in the associated windings, Umans: 2014 [146]. Ordinarily, a drop in coercivity (negative $H_{c}$ ) in a typical PM demagnetisation curve is indicative of proportional PM temperature rise. Although, according to Sjökvist (2014) [32], this generalisation is not applicable to ferrites and Alnico because of their positive temperature coefficient which mean that they perform better as they get hotter. Besides, ferrites, unlike rare-earths, are not prone to eddy current losses, also meaning that their electrical resistance is very high, Nipp: 1999 [57]. This goes to show that the PM quality is also a factor of its demagnetisation characteristics.

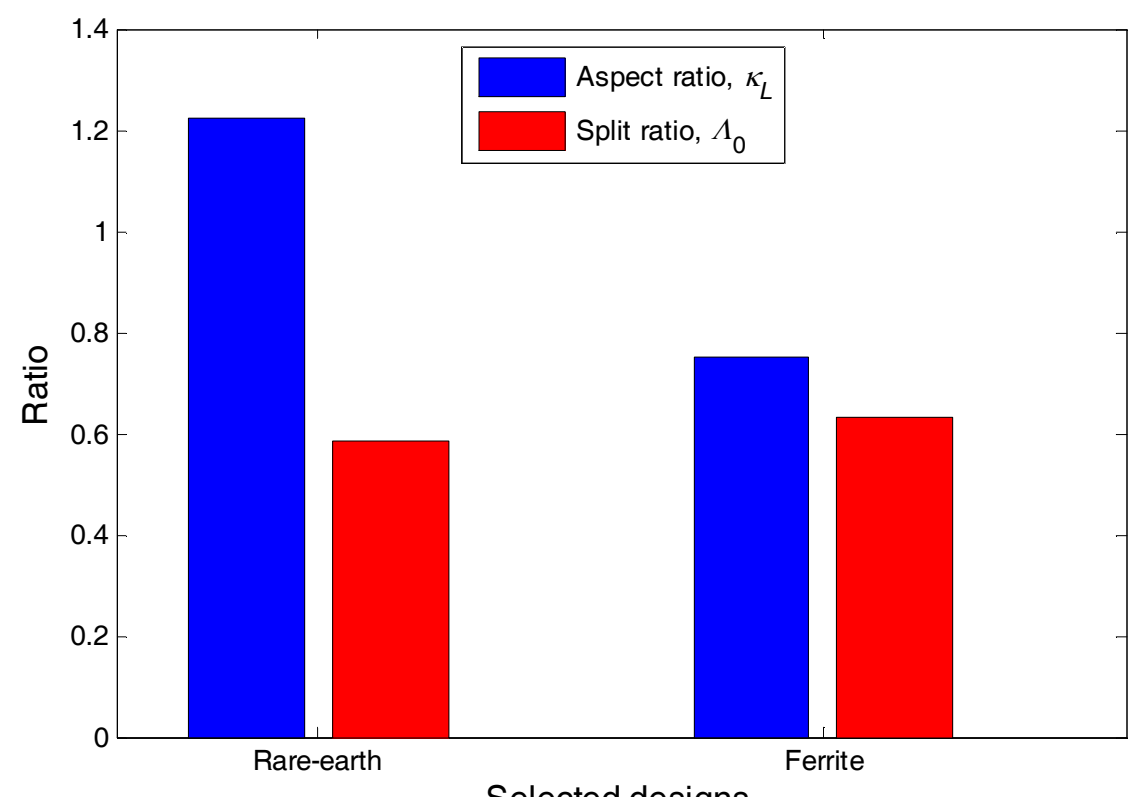

Fig. 4.10. Comparison of aspect ratio and split ratio in optimal PM-FSM variants.

Table 4.6. Performance indicators

\begin{tabular}{c|c|c|c}
\hline Parameters & Units & Rare-earth & Ferrite \\
\hline \hline Average torque, $\tau_{e}$ & $\mathrm{Nm}$ & 259.23 & 258.48 \\
Torque ripple, $\kappa_{\delta}$ & $\%$ & 7.84 & 9.42 \\
Cogging torque & $\%$ & 6.42 & 12.65 \\
Stator iron mass & $\mathrm{kg}$ & 23.84 & 36.42 \\
Rotor iron mass & $\mathrm{kg}$ & 10.02 & 18.91 \\
Copper mass & $\mathrm{kg}$ & 6.95 & 9.19 \\
PM mass, $M_{P M}$ & $\mathrm{~kg}$ & 6.33 & 12.07 \\
Active mass, $M_{A}$ & $\mathrm{~kg}$ & 49.02 & 80.27 \\
Copper loss, $P_{C u}$ & $\mathrm{~W}$ & 447.92 & 341.63 \\
Core loss, $P_{C o r e}$ & $\mathrm{~W}$ & 188.17 & 163.15 \\
Output power, $\mathrm{P}_{\text {out }}$ & $\mathrm{kW}$ & 9.990 & 9.972 \\
Machine efficiency, $\eta$ & $\%$ & 94.014 & 95.182 \\
Power factor, $\mathrm{PF}$ & - & 0.797 & 0.797 \\
Torque/Active mass & $\mathrm{Nm} / \mathrm{kg}$ & 5.50 & 3.37 \\
\hline
\end{tabular}


In this subsection, the demagnetisation tolerance of the rare-earth and ferrite PM-FSM benchmarks (DI and DII, respectively) are tested in 2-D static FEA procedure, in order to appraise the impact of the load current characteristics on their PM remanence $\left(B_{r}\right)$. The characteristics of the ferrite and rare-earth PMs used for the investigation are provided in Table 4.7. The demagnetisation characteristics of both machines are investigated using the average flux density component normal to the field lines along the $\mathrm{x}$-direction, illustrated by points " $\mathrm{A}$ " and "B" in Fig. 4.12. As stated in Fasolo, Alberti and Bianchi (2014) [69], the safety limit of flux density normal to PM flux lines in rare-earth $\mathrm{PM}$ is given as $B_{n}>0.4 \mathrm{~T}$ at maximum temperature of $120^{\circ} \mathrm{C}$, while that of ferrite $\mathrm{PM}$ is $B_{n}>0.1 \mathrm{~T}$ at minimum temperature of $20{ }^{\circ} \mathrm{C}$. The PM position where the magnitude is lowest (the so-called 'worst-load point') for a given static FEA solution is thus considered. But, it should be mentioned that the demagnetisation levels considered in this study are mainly evaluated based on the magnetic saturation effects as a result of the load current coupling with the PMs.

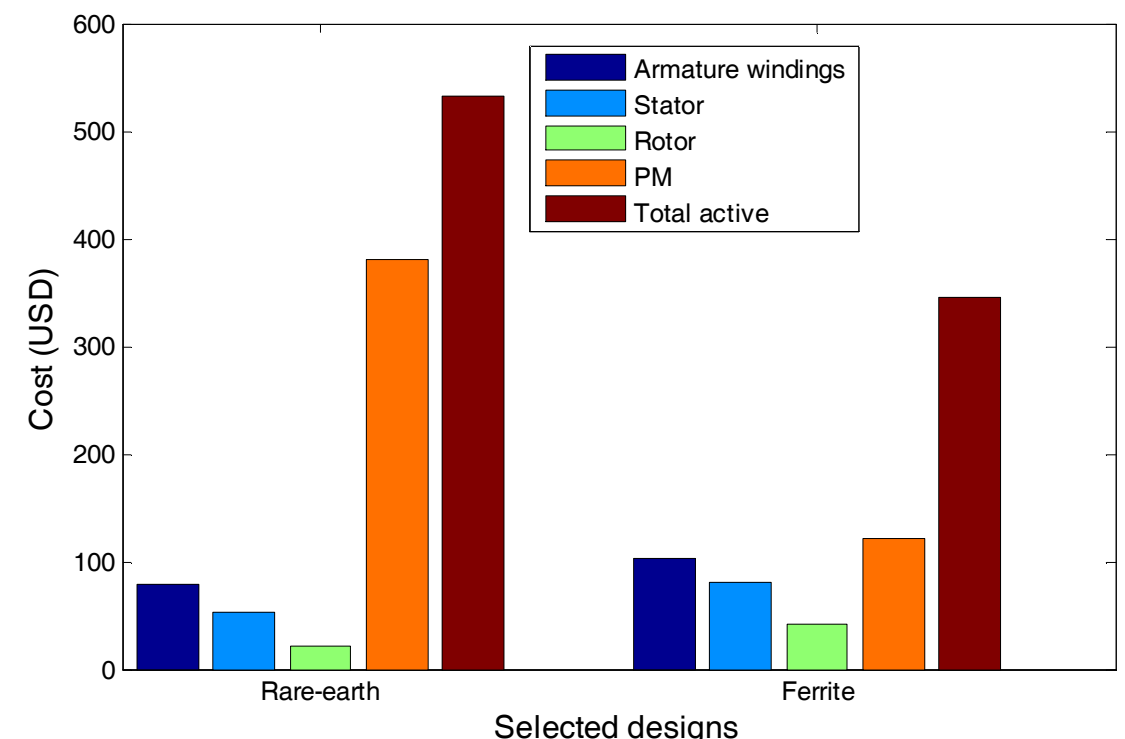

Fig. 4.11. Cost comparison of different material components.

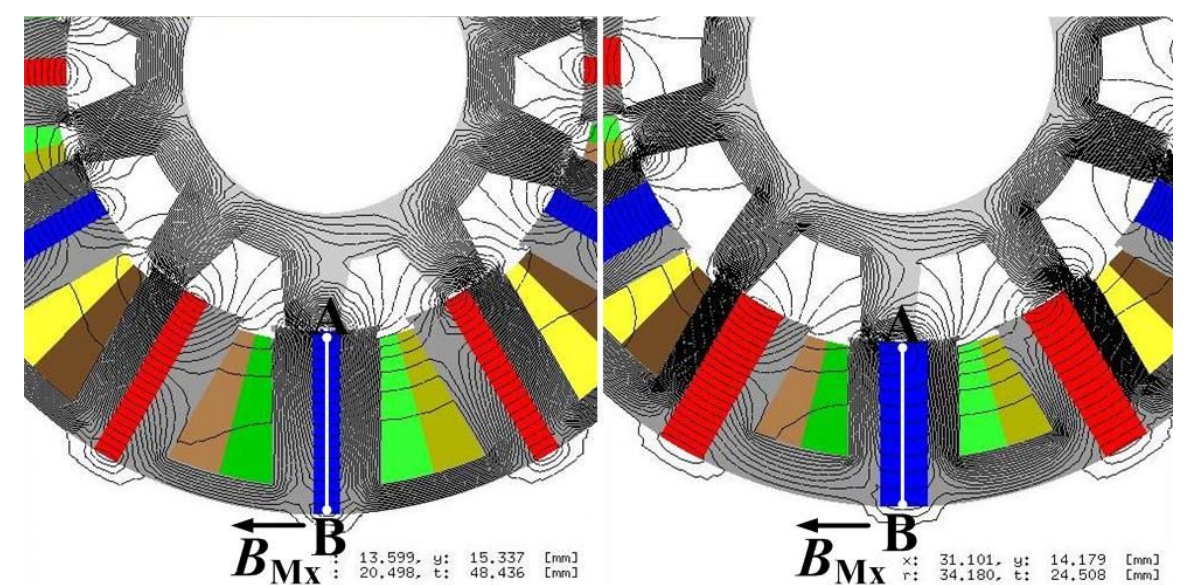

(a)

(b)

Fig. 4.12. 2-D static FEA display of PM reference point normal to flux lines along the $\mathrm{x}$-direction for: (a) rare-earth PM-FSM, (b) ferrite PM-FSM. 
Table 4.7. Characteristics of selected PMs

\begin{tabular}{c|c|c}
\hline & Ferrite & Rare-earth \\
\hline \hline Coercive force, $H_{c}(\mathrm{kA} / \mathrm{m})\left(\right.$ at $\left.20{ }^{\circ} \mathrm{C}\right)$ & 258 & 900 \\
PM remanence, $B_{r}(\mathrm{~T})$ & 0.4 & 1.2 \\
Mass density $\left(\mathrm{kg} / \mathrm{m}^{3}\right)$ & 5000 & 7500 \\
Relative permeability, $\mu_{r}$ & \multicolumn{2}{|c}{1.06} \\
\hline
\end{tabular}

In Fig. 4.13, it is shown that across the load region considered, the rare-earth design is unable to satisfy the safety limit (showing $B_{\mathrm{Mx}}<0.4 \mathrm{~T}$ ), unlike the ferrite design (showing $B_{\mathrm{Mx}}>0.1 \mathrm{~T}$ ). The main reason for this disparity is due to the high cross-magnetisation effects of the current-induced magnetic fields in the former, which results in the higher armature-reaction voltage values observed between the margin of no-load and about the nominal load as shown in Fig. 4.14.

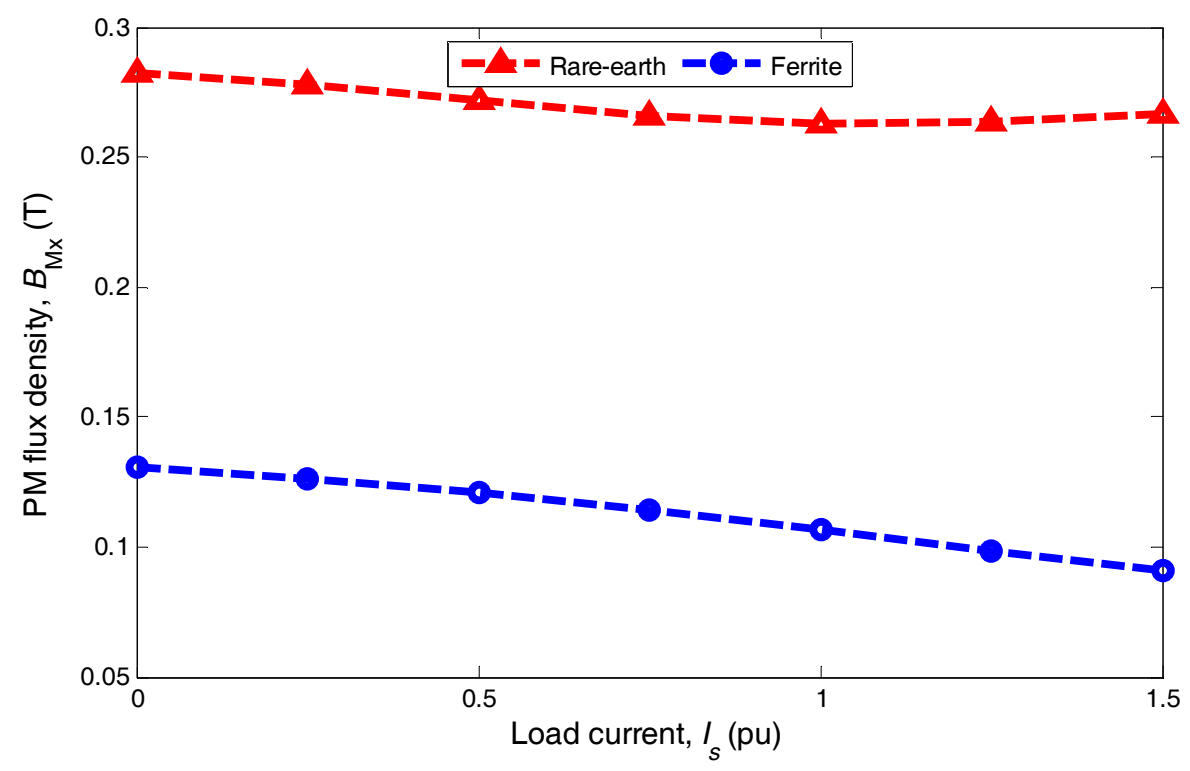

Fig. 4.13 Average PM flux density under different load conditions.

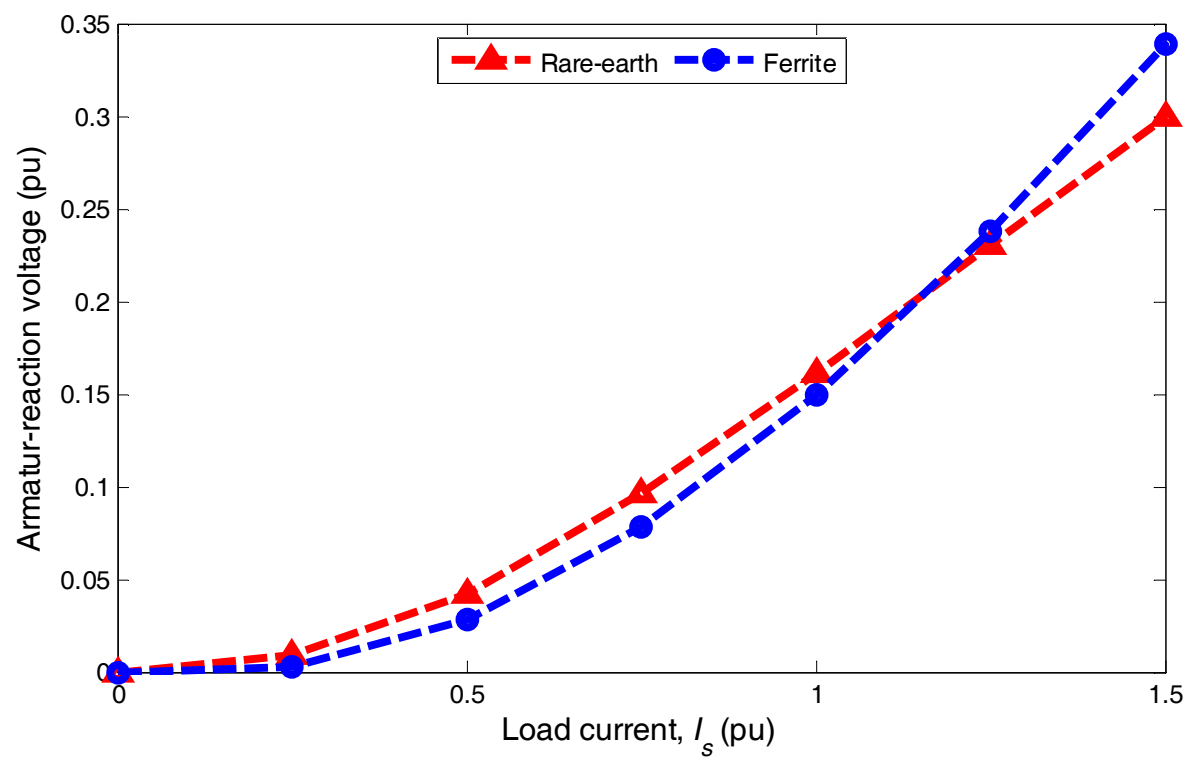

Fig. 4.14. Load profile of normalised armature-reaction voltages at $360 \mathrm{r} / \mathrm{min}$. 
On the other hand, beyond the nominal load in Fig. 4.13, the safety limit for the ferrite design is not respected. Apparently, this is due to drastic increase in saturation when overloaded. Meanwhile, the presented contour plots in Fig. 4.15 are zoomed in to clearly show the degree of demagnetisation, at rated conditions, on the flux density levels inside the PMs; thus, corroborating the trend observed in Figs. 4.13 and 4.14.

With current induced magnetic fields, the transition of the mean PM flux density in Fig. 4.13 also implies steady but drastic demagnetisation behaviour of the ferrite PM machine. Thus, it can be said that the ferrite PM-FSM can operate with minimum demagnetisation risks at load current values not exceeding nominal condition. Suffice to say that the emphasis on the nominal condition is based on the fact that such operating regime is the most dominant range between the cut-in and cut-out values in wind speed bands of variable-speed wind turbines, Simões, Farret and Blaabjerg: 2015 [143]. Nevertheless, the deep saturation of the rare-earth PM machine, within the same load regime, is an indication of higher demagnetisation risks.

Meanwhile, by taking into account the fact that the eddy current effects on rising temperature are not considered in the 2-D static FEA simulations, the demagnetisation study is nonetheless supported by the following assumptions:

- By increasing the load current, the external magnetic fields necessary to demagnetise the PM is mimicked. Thus, the potential of irreversible demagnetisation is exacerbated by the heating up (temperature rise) of the windings when loaded, Zhou et al: 2012 [126].

- In any case, the results may not differ compared to transient FEA solution because the temperature coefficient of ferrites is much lower than that of rare-earths, Kudrjavtsev et al: 2015 [127].

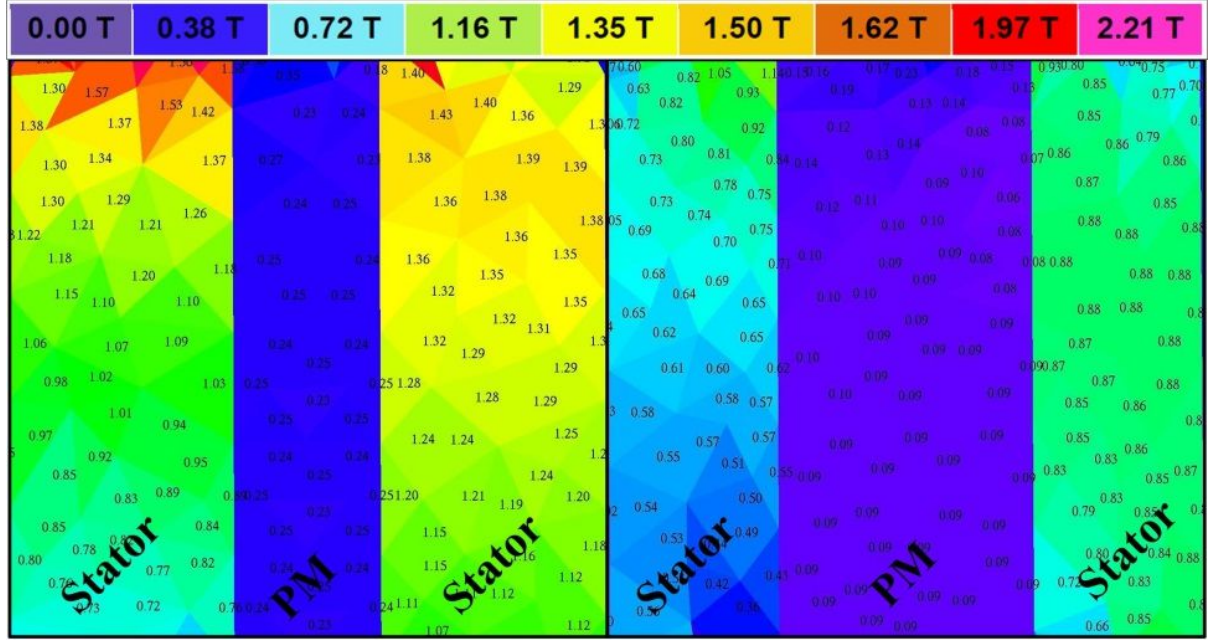

(a) (b)

Fig. 4.15. Contour plots of PM flux densities at rated conditions: (a) rare-earth, and (b) ferrite. 
- Similarly, Fasolo, Alberti and Bianchi (2014) [69] also portrayed the demagnetisation behaviour of the worst-load at rated condition, and along stationary points using 2-D FEA, as well as Kang et al (2003) [128], who used 2-D magneto-static FEA in their study.

- Lastly, transient FEA design is subsequently earmarked for comparing the 2-D static FEA results, and with good confidence reported.

\subsubsection{Transient FEA Solutions}

So far, the results in this section have been based on non-linear static FEA method carried out by means of an in-house 2-D FEA package called SEMFEM, Gerber: 2011 [91]. To this end, counterpart 2-D and 3-D transient FEA designs are constructed in ANSYS Maxwell ${ }^{\odot}$ environment to contrast with the 2-D static FEA modelling. Consequently, Figs. 4.16 and 4.17, which compare the noload flux linkages, are realised as shown.

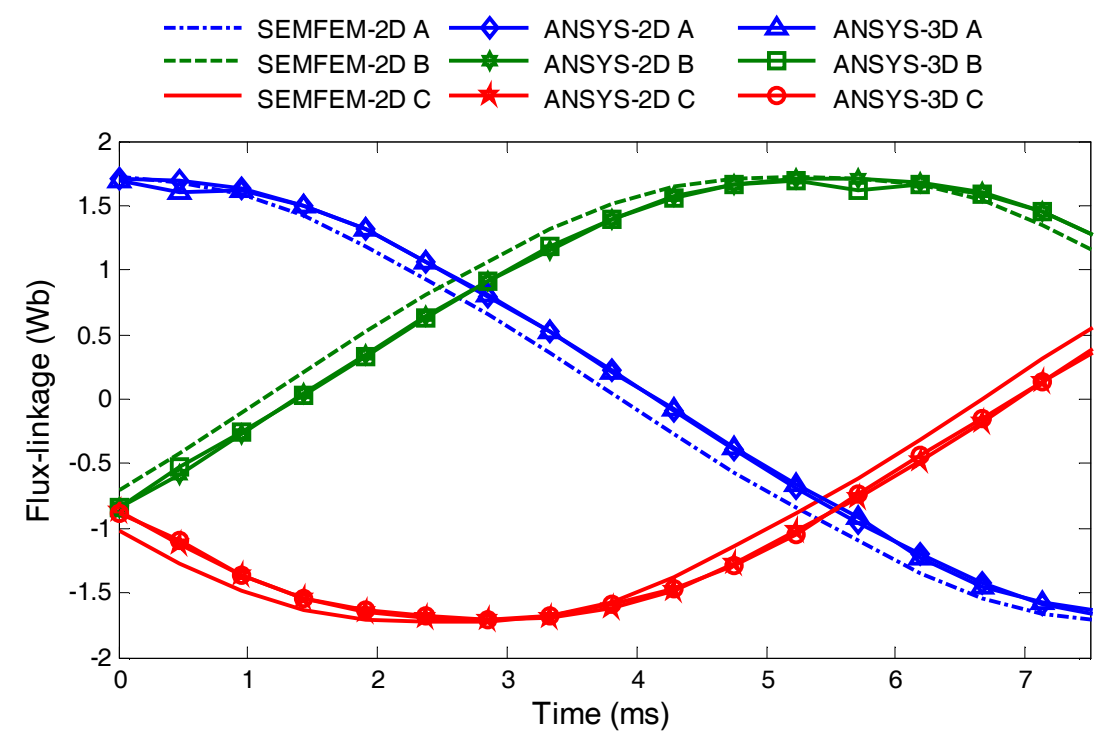

Fig. 4.16. Comparison of flux linkages in rare-earth PM-FSM under no-load conditions.

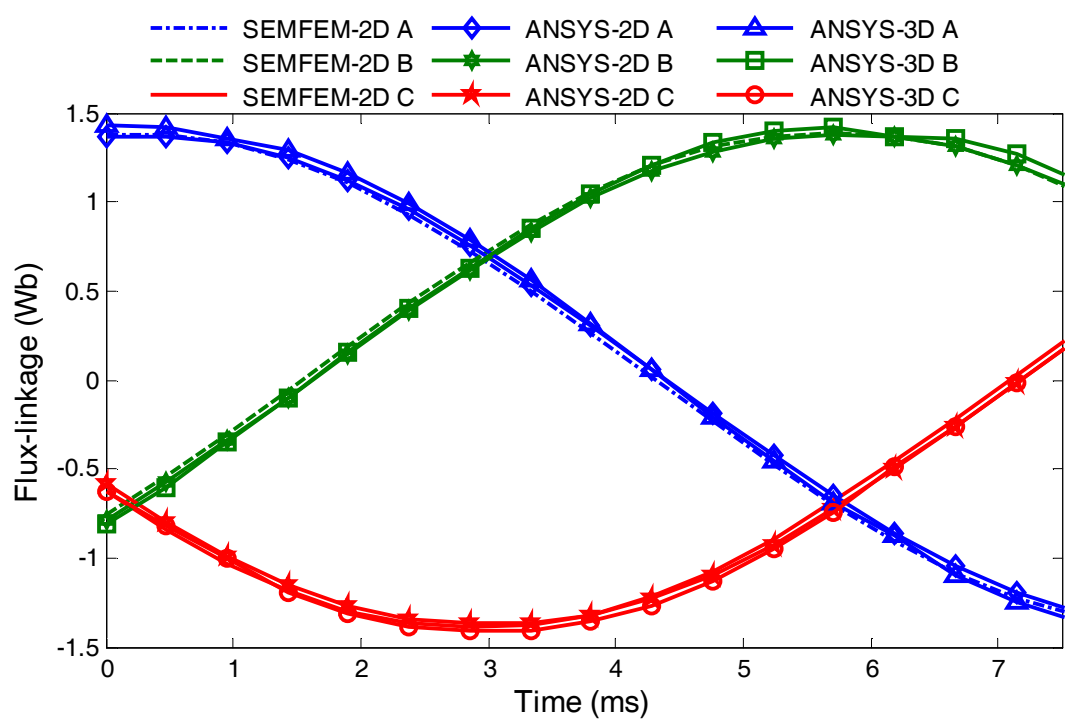

Fig. 4.17. Comparison of flux linkages in ferrite PM-FSM under no-load conditions. 
It is observed that, based on the comparison of the different FEA techniques, an approximate correlation has been obtained. The confidence in the FEA transient solutions is in the fact that the end-winding effects do not seriously differentiate the 3-D and 2-D solutions. As a matter of fact, when evaluated at rated conditions (not shown), the $2-\mathrm{D}$ results match that of $3-\mathrm{D}$ by $100 \%$ for the rare-earth design and $96.7 \%$.

\subsection{Chapter Summary}

In this chapter, the possibility of using rare-earth-free field excitation sources, viz., ferrite PM and wound-field, based on the wind generator design of the 12/10 FSM is initiated. To facilitate the design process, non-gradient-based design optimisation is processed on three design objectives (minimum mass of the field sources, minimum total active mass and minimum torque ripple), as well as three constraints (power factor, electromagnetic torque and efficiency).

Based on the outcome of the multiple objective evaluations, a matrix of the cross-interactions of the three parameters which evinced as Pareto optimal fronts for each of the specified design problems, showed that the active mass of ferrite PM-FSMs are significantly minimised compared to the WF-FSMs. In addition, it was observed that an increase in the mass of the ferrite PM result in decrease in the torque ripple, whereas the mass of the wound-fields increases with respect to decrease in torque ripple values at two times more than in the former. Besides, it was shown that by slightly increasing the total active mass of the WF-FSM, the torque ripple can also be significantly improved.

In general, it was found that the opposing design and performance characteristics of the optimal ferrite PM-FSM and WF-FSM candidates are perpetrated as tradeoffs in cocktails of their split and aspect ratios. The aspect ratio batch of the ferrite PM-FSMs, as with typical PM machines, are significantly higher when compared to that of the WF-FSMs. On the other hand, the WF-FSM designs, due to the incorporation of field coils, viz., the tendency for higher copper losses, result in a consignment of larger split ratios.

Informed by the pool of optimal design candidates, individual selections which were made to further compare the optimal performance of the ferrite PM-FSM against WF-FSM indicate that for the same output power constraint, the former is superior in terms of efficiency ( $4.2 \%$ higher), torque ripple (55.2\% lower) and torque density (181.25\% higher), at only half the total material cost of the WF-FSM.

Encouraged by the excellent performance of the ferrite PM-FSM for the proposed wind generator drive, another study is instituted to investigate its performance and demagnetisation risks in comparison to a similarly designed 12/10 rare-earth PM-FSM. Armed with a new set of design tar- 
gets (two objective functions and four design constraints), the resulting population of smaller sized rare-earth PM optimal design candidates, compared to the ferrite PM-FSM solutions, was never in doubt. Thus, based on two individual nominations as before, the rare-earth design excelled above the ferrite PM design in terms of torque ripple (16.77\% lower), cogging torque $(49.24 \%$ lower) and torque density (63.20\% higher). However, a slight advantage in terms of efficiency (1.24\%), and a major cost savings of $35 \%$ was recorded in the latter. The higher total material cost of the rare-earth PM-FSM is mainly escalated by the high cost of the rare-earth PM materials despite appearing in a smaller size cum mass.

On the other hand, it was further discovered that deeper saturation occurs in the rare-earth design, which lowers its demagnetisation withstand ability, even below nominal load conditions. On the contrary, the ferrite design can only experience such high demagnetisation risks at load current values above rated. This is a key finding especially as ferrite PM, with a lower energy potential, is notorious for its high demagnetisation risks. Thus, for the proposed geared MS wind generator drive, ferrite PM-FSMs possess the necessary qualities to be considered as a low-cost and robust option. On the other hand, the candidacy of WF-FSMs is not to be entirely discarded because, despite being the most expensive and least performing among the three designs, it presents no risk of demagnetisation and can also provide flux control based on the time-varying nature of the wind energy resource. 


\section{Chapter Five}

\section{Formulation and MULti-ObJective Design Optimisa- TION OF WF-FSM WIND GENERATORS}

In this chapter, an in-depth study is undertaken on the multi-objective design optimisation (MDO) of a rare-earth-free FSM, specifically in terms of the 12/10 wound-field flux switching machine (WFFSM). This study differs from the one carried out in the preceding chapter because it specifically focuses on diverse MDO strategies and their influence on the wind generator drivetrain performance and cost at $10 \mathrm{~kW}$ power. Besides, great emphasis is placed on the design formulation theory of such WF-FSMs, which is not as matured as that of PM-FSMs. For the proposed wind generator drive, the MDO processes are fully discussed, as per their potentials for producing Pareto optimal solutions, which enable the designer to have a cocktail of optimal design options. The optimisation is tackled as two different problem formulations thanks to a 2-D static FEA procedure, supported by simple analytic formulations. Eventually, two optimal designs are randomly apprehended and compared, each from the different MDO problems established. Thereafter, 3-D transient FEA is prescribed as a means of testing the accuracy of the 2-D static FEA results obtained. Meanwhile, the work reported in this chapter has been published as a journal contribution in Akuru and Kamper (2017) [125].

\subsection{Introduction}

Mostly driven by technological revolution and LCOE reductions in recent times, wind power is becoming established as the leading source of renewable energy for electricity demand, REN21: 2016 [1]. Similarly, electrical machines which are a major component in wind generator drives have attracted corresponding attention, both from researchers and the industry, Polinder et al: 2013 [7], Zhu and $\mathrm{Hu}: 2013$ [10] and El-Refaie: 2016 [121]. The commonest electrical machines in use for wind energy drives are DFIGs and PMSGs. Although DFIGs and PMSGs have become established for wind applications, the former are notorious for high maintenance costs, whereas the issue with the latter is the high cost of its high-grade PMs used in their manufacturing, to mention just a few. To overcome these issues, most researchers have become concerned with the use of non-conventional electrical machines for wind energy drives, e.g. stator-active brushless machines, Cheng et al (2011) [44].

FSM as an example of stator-active brushless machines, which recently resurfaced after initially being proposed by Rauch and Johnson (1955) [40], is gradually gaining a foothold in wind energy 
applications, Ojeda et al: 2012 [37], Lin et al: 2011 [74], Dobzhanskyi et al: 2012 [75], Ditmanson et al (2013) [84] and Shao et al: 2016 [122]. To this end, these studies have predominantly been on PM-FSMs with high-grade PMs and for low-speed (LS) systems. On the other hand, there are WFFSMs. The advantage of WF-FSMs over DFIGs is that they do not require slip rings and brushes, and they can be better managed thermally because of the position of their field coils in the stator. With regards to PMSGs and PM-FSMs, WF-FSMs are not only a probable low-cost option but their fields can directly be controlled, and they come without any risk of demagnetisation.

In this chapter, WF-FSM is presented as a suitable candidate for the geared medium-speed (MS) wind generator drive illustrated in Fig. 1.12 in subsection 1.3.3. In contrast to previous published studies and the preceding chapter, this study is focused on the integrated design formulation for the constrained MDO of these machines. With the insights provided in the earlier parts of the preceding chapters which introduce the MDO approach, to the best of the researcher's knowledge, no other study has been reported for WF-FSMs based on the methodology of optimising a cocktail of different machine performance parameters in tandem with the drivetrain costs.

For example, in Zhou and Zhu (2014) [70] different topologies (12-slots/8-poles, 12-slots/5poles, 12-slots/7-poles, and 9-slots/5-poles) of the WF-FSM were optimised and compared using deterministic optimisation method with respect to maximum average torque for high torque density applications Whereas in Tang et al (2012) [67], a proposal is made (without design optimisation) to replicate the popular 12-slot/14-poles PM-FSM topology after the WF-FSM fashion, thus illustrating adjustable field and comparable torque capabilities. In some of the other studies, where the emphasis is on automotive drive applications, the focus has been primarily on the maximum torque/power capabilities (with/without design optimisation) in contrast with PM-FSMs, Raminosoa et al: 2015 [71], Sulaiman, Kosaka and Matsui: 2012 [95], Kosaka et al: 2014 [123] and Tang et al: 2013 [119]. Besides, in most of these studies, the articulation of the design process is also not emphasised.

To this end, the current study is the first dedicated design analyses on the proposed 12/10 WFFSM topology, which begins by rehearsing the basic facts on the geometrical conceptualisation of WF-FSMs based on the popular sizing technique used in PM-FSMs. Thereafter, the MDO process of some critical wind generator performance quantities is undertaken in 2-D FEA simulations. The optimisation process is employed using a stochastic algorithm which automatically resolves the optimum solution set into a Pareto optimal front. Subsequent subsections are then used to present the results, discussions, as well as 3-D FEA solutions, primarily based on some chosen optimal design candidates. Lastly, a conclusion on the findings is given in the last section of the chapter. 


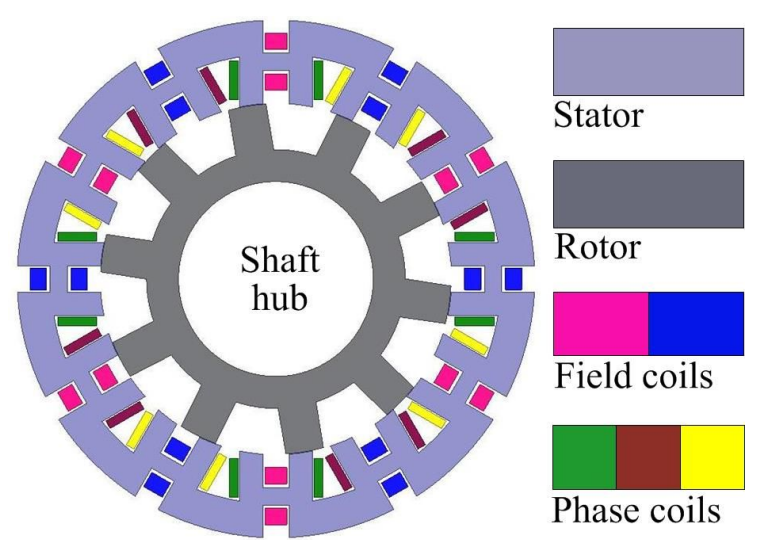

Fig. 5.1. Cross-sectional view of 12-slots/10-pole WF-FSM topology.

\subsection{WF-FSM Geometry Development}

The operating principles of FSMs have been fully represented in the literature, such as in Rauch and Johnson (1955) [40], Hua et al (2006) [61], Tang et al (2012) [67], Zhou and Zhu (2014) [70] and Raminosoa et al (2015) [71]. Also, Appendix A1 has been developed to fully discuss the fundamentals in the operation of FSMs.

In retrospect, FSMs are unique double salient pole machines with robust rotor structure, having bipolar and sinusoidal phase flux linkages, flux focusing capabilities and high torque densities as highlighted in Cheng et al (2011) [44]. It can be designed to reflect radial-flux direction as in Rauch and Johnson (1955) [40], transverse-flux direction as in Dobzhanskyi et al (2012) [75] or axial-flux direction as in Lin et al: 2011 [74], with the radial-flux design presenting the simplest topology. Consequently, the most popular radial-flux PM-FSM topology, the 3-phase 12-slots/10-poles (12/10) machine, which when redesigned with wound-fields, is as presented in Fig. 5.1.

The presentation in Fig. 5.1 is thus preferred in the current analyses, which is based on its design and optimisation for geared MS wind generator drives. The generator MS drivetrain is implemented at $360 \mathrm{r} / \mathrm{min}$ rated speed calculable from (2.14) in section 2.2 in Chapter 2. Based on this speed, a low-cost, 1- or 2-stage gearbox, with a proposed gearbox ratio of 10-40 could suffice as the medium of transmission between the turbine rotor and the generator shaft in a typical wind turbine.

Note that, inasmuch as other radial-flux WF-FSM topologies exist as highlighted in Zhou and Zhu (2014) [70], the structure in Fig. 5.1 is, without doubt, ahead in terms of optimum phase/field slots combinations per torque density as inferred in Tang et al (2012) [67]. The design targets of the current study, for the different optimisation problems to be solved, are presented in Table 5.1. To this end, the sizing to power expression described in Appendix A1.2 is used to produce the initial design from which other design parameters such as the stator slot opening width $\left(b_{s l s}\right)$, stator pole width $\left(b_{p s}\right)$, stator yoke height $\left(h_{y s}\right)$, rotor pole width $\left(b_{p r}\right)$, and rotor yoke height $\left(h_{y r}\right)$ are initiated. 
Table 5.1. Design requirements

\begin{tabular}{c|c|c|c}
\hline Symbol & Limits & Target 1 & Target 2 \\
\hline \hline $\mathrm{P}_{\text {out }}(\mathrm{kW})$ & output power $\geq$ & 10 & 10 \\
$\eta(\%)$ & efficiency $\geq$ & 88 & 88 \\
$\theta_{\mathrm{sF}}$ & slot fill factor for field windings & 0.45 & 0.45 \\
$\theta_{\mathrm{sp}}$ & slot fill factor for phase windings & 0.45 & 0.45 \\
$\kappa_{\delta}(\%)$ & torque ripple $\leq$ & 10 & 15 \\
$\mathrm{PF}$ & power factor $\geq$ & 0.8 & 0.9 \\
\hline \multicolumn{3}{|c}{}
\end{tabular}

\subsection{WF-FSM Analytical Modelling}

\subsubsection{Steady-State Equations}

As usual, the MDO will be processed by means of a 2-D static FEA program (SEMFEM), coupled to an optimiser (VisualDoc). To this end, the steady-state direct axis (d-axis) and quadrature axis ( $\mathrm{q}-$ axis) (a.k.a. dq axes) equations, already established for generator mode in section 2.2 in Chapter 2 and subsection 4.2.1 in Chapter 4, are applied via Python scripts linked to the FEA program.

\subsubsection{End-winding calculations}

The temptation to ignore the end-effects in FSMs might arise because in PM-FSMs, the endwindings are perceived as short. However, the presence of field coils and its arrangement over the phase coils in the WF-FSM topology being considered in this study makes it necessary to formulate an approximation for the end-winding effects. Also, for the sake of the optimisation process, an analytical approximation of the end-winding effect is as important as the convergence to the true optimal design.

Consequently, the method of considering only the resistance and ignoring the reactance as done in Zhou and Zhu (2014) [70] is unsatisfactory for a design optimisation process of the WF-FSM because at some point in the design space, the end-winding reactance may portend very significant impact on the optimum design. Also, by prescribing a fixed margin to account for end effects as done in Raminosoa et al (2015) [71] is limited because the design optimisation process of WF-FSMs poses a nonlinear problem. Hence, an approximate formula as devised for non-overlap PM winding machines in Potgieter and Kamper (2014) [94] is refined to activate the real-time analytic calculations of the end-winding inductance during FEA design optimisation process. Calculations for the endwinding resistance are also captured by means of the end-winding approximations. Initial results are presented to validate the accuracy of the procedure.

Fig. 5.2 shows how most of the WF-FSM end-winding parameters are devised from different cross-sectional viewpoints. From previous referrals in sections 2.2 and 2.3, as well as subsection 4.2.1, the phase and field resistances, now with end-effects, are expressed as 


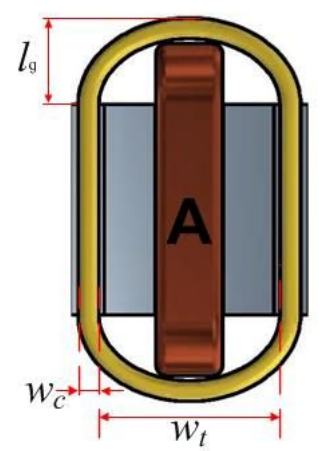

(a)

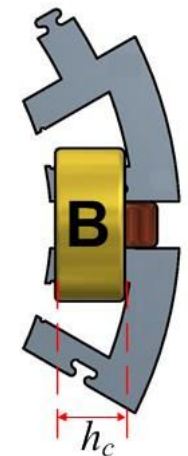

(b)

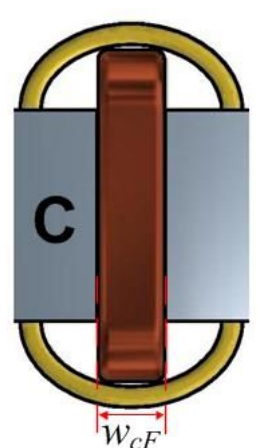

(c)

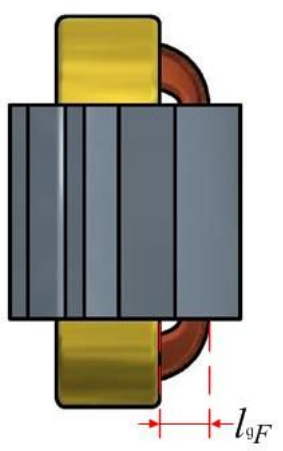

(d)

Fig. 5.2. Different WF-FSM end-winding projection: (a) airgap side, (b) radial cross-section, (c) outer perimeter surface and (d) axial cut-out (Parts: $\mathbf{A}=$ field coil, $\mathbf{B}=$ phase coil, $\mathbf{C}=$ stator laminations).

$$
\begin{aligned}
& R_{S\left(l_{e 1}=w_{t}+2 w_{c}+2 l_{g}\right)}=2 q N_{p h}^{2} \rho_{C u} \frac{l_{s t}+l_{e 1}}{A_{p h}}, \\
& R_{F\left(l_{e 2}=2\left(l_{g}+w_{c F}\right)\right)}=2 q_{F} N_{F}^{2} \rho_{C u} \frac{l_{s t}+l_{e 2}}{A_{F}},
\end{aligned}
$$

where $l_{e 1}$ is the end-winding length on one side of the phase coil, $w_{t}$ is the average tooth width, $w_{c}$ is the phase coil width, $l_{9}$ is the full distance of the phase end-winding from the lamination stack, $l_{e 2}$ is the end-winding length on one side of the field coil, $l_{9}$ is full the distance of the field end-winding coil from the lamination stack, and $w_{c F}$ is the field coil width.

As earlier indicated, calculations for the end-winding inductances are also formulated based on the method described in Potgieter and Kamper (2014) [94] as

$$
\begin{aligned}
& L_{e(1)\left(a=0.5 l_{e}, b=h_{c}, c=w_{c}\right)}=\frac{1.937}{n_{a}^{2}} \frac{2 a^{2}}{b} N_{p h}^{2} q K \quad(\mu \mathrm{H}), \\
& L_{e(2)\left(a=0.5 l_{e}, b=2 w_{c}, c=h_{c}\right)}=\frac{1.257 l_{e}}{n_{a}^{2}} \frac{2 a}{b} N_{p h}^{2} q K \quad(\mu \mathrm{H}), \\
& L_{e}=K_{M}\left(L_{e(1)}+L_{e(2)}\right) \quad(\mu \mathrm{H}),
\end{aligned}
$$

where $l_{e}=w_{t}+w_{c}, w_{t}$ and $w_{c}$ are as previously defined, $h_{c}$ is the height of the phase coil, $n_{a}$ is the number of parallel circuits, and $K$ is a constant as designated in Potgieter and Kamper (2014) [94]. The expressions in (5.3) and (5.4) are when $l_{9}>2.5 \mathrm{~mm}$. The variables $a, b$, and $c$ are used to determine $K$, while $K_{M}$ is a factor required to account for high mutual phase coupling effects.

Table 5.2. Analytic calculations vs. FEA results for sampled $10 \mathrm{~kW}$ WF-FSM

\begin{tabular}{c|c|c|c}
\hline & $\boldsymbol{L}_{\boldsymbol{d}}$ & $\boldsymbol{L}_{\boldsymbol{q}}$ & $\boldsymbol{P}_{\boldsymbol{c} \boldsymbol{u}}$ \\
\hline \hline 3-D FEA & $173.725 \mu \mathrm{H}$ & $169.863 \mu \mathrm{H}$ & $853.110 \mathrm{~W}$ \\
2-D FEA & $152.632 \mu \mathrm{H}$ & $149.056 \mu \mathrm{H}$ & $497.102 \mathrm{~W}$ \\
Actual $L_{e}(3-D$ FEA $-2-D$ FEA) & $21.093 \mu \mathrm{H}$ & $20.807 \mu \mathrm{H}$ & $356.007 \mathrm{~W}$ \\
Analytic $L_{e}$ (with $\left.K_{M}=1.5\right)$ & $18.764 \mu \mathrm{H}$ & $18.764 \mu \mathrm{H}$ & $336.691 \mathrm{~W}^{20}$ \\
Deviation & $11.041 \%$ & $10.326 \%$ & $5.425 \%$ \\
\hline
\end{tabular}

\footnotetext{
20 Value not multiplied by $K_{M}$.
} 
It has to be mentioned that the end inductance effect is not applied to the field windings by assuming a non-periodic characteristics for their associated currents in 2-D static FEA. Also, to establish the accuracy of the emulated analytic expressions for the end effect evaluations, the difference between the dq-axes inductance in 2-D FEA (without end effects) and 3-D FEA (with end effects) is taken as the actual end-winding inductance. Thus, for a random $10 \mathrm{~kW}$ WF-FSM sample design, the actual versus analytic evaluations as devised in (5.1), (5.2) and (5.5) are compared in Table 5.2. The copper losses compared are in terms of the total end-winding losses highlighted as shown. Although the analytic formulations fall behind by some margins when compared to the actual 3-D effects, the approximation is nevertheless beneficial in terms of speed and nonlinearity for the proposed WFFSM MDO problem.

\subsection{Multi-Objective Design Optimisation}

\subsubsection{Optimisation Procedure and Problem Formulation}

As implied in Zhang, Ionel and Demerdash (2016) [89], the design optimisation of electrical machines is a nonlinear process, which leads to discontinuity for gradient-based (deterministic) solutions. Hence, a constrained multi-objective approach presents the best solution for any given problem because it allows each objective to formulate the right partnership (compromise) in the final optimum design. The basic outcome of such design optimisation process is a set of optimal solutions known as the Pareto optimal set.

A number of the objectives which may be sought for in the design optimisation of wind generators include maximum power factor, minimum torque ripple, and lowest cost or minimum mass, a combination of these for the design optimisation of WF-FSMs, which is yet to be reported. In this paper, FEA-based MDO reinforced by analytical formulations is undertaken in a highly efficient evolutional algorithm - the non-denominated sorting genetic algorithm (NSGA-II) - to predict the behavior of WF-FSMs for wind energy drives. The design optimisation workflow process is as illustrated already in Fig. 1.13 in section 1.5 in Chapter 1.

NSGA-II is an adaptive search technique inspired from nature and works on the principle of Darwin's theory of the survival-of-the-fittest, otherwise broadly referred to as evolutionary algorithms. According to its designers - Deb et al (2002) [107], it works with a set of solutions (population) and as the simulation (evolution) proceeds, the individuals in the population improve.

NSGA-II is a fast and elitist multi-objective algorithm which works on the principle of nondominated sorting by using two-tier fitness assignment technique-primary and secondary fitness. The primary fitness is evaluated based on domination level while the secondary fitness is evaluated 
based on the diversity of the solution in its domination level. The two operators used to express domination ranks are crossover probability, $P_{c}$ and mutation probability, $P_{m}=1 / n$ (where $n$ is the number of design variables), while the distribution indexes for crossover $\left(\eta_{c}\right)$ and mutation $\left(\eta_{m}\right)$ are parameters used for the control of diversity. The highlight of NSGA-II algorithm is that it produces an optimal solution set, whereby none of the solutions in the optimal solution set dominate because they are equally useful to the specific choice of the machine designer. However, it must be said that a major limitation in the adoption of NSGA-II algorithm in conjunction with FEA for the analyses of electrical machines is the huge computation memory and time expended e.g., the simulation time for a single FEA solution, in the present study, instance is around $100 \mathrm{~s}$.

On the other hand, the criteria used to determine the design constraints and set of objectives is incumbent on the proposed application of the WF-FSM design. Thus, to design a suitable wind generator, the following considerations are important:

I. The generator head mass is desired to be made as light as possible, especially since the presence of the field coils tend to create high split ratios which increases the size of WF-FSMs, Akuru and Kamper: 2016 [111]. Hence, improving the power density of the wind generator by minimising the generator mass will result in lower manufacturing costs.

II. The field losses in WF-FSM tend to worsen its efficiency performance, Raminosoa et al: 2015 [71].

III. Because FSMs are generally inverter-fed machines as highlighted in Hua et al (2006) [61], a high power factor is very critical to the size and cost of the solid state converters (SSCs), with effects on the overall drivetrain performance, Sulaiman, Kosaka and Matsui: 2012 [95].

IV. Very low torque ripple is critical to the survival of the drivetrain, because it is a source of mechanical stress, Xia et al: 2011 [124]. Besides, FSMs are generally known to suffer from high torque ripples, Cheng et al (2011) [44].

To this end, the MDO process is posed in two different problems ${ }^{21}$ as follows:

1: $\min \left\{F(\mathbf{x})=\left[M_{F}(\mathbf{x}), M_{A}(\mathbf{x})\right]\right.$, s.t. $\left\{G(\mathbf{x})\left[\mathrm{P}_{\text {out }}(\mathrm{x}) \geq 10 \mathrm{~kW}, \mathrm{PF}(\mathbf{x}) \geq 0.8, \kappa_{\delta}(\mathbf{x}) \leq\right.\right.$ $10 \%, \eta(\mathbf{x}) \geq 88 \%]$,

2: $\min \left\{F(\mathbf{x})=\left[1 / \mathrm{PF}(\mathbf{x}), \kappa_{\delta}(\mathbf{x})\right]\right.$, s.t. $\left\{G(\mathbf{x})\left[\mathrm{P}_{\text {out }}(\mathbf{x}) \geq 10 \mathrm{~kW}, \eta(\mathbf{x}) \geq 88 \%\right],(5.7)\right.$ where $F(\mathbf{x})$ is a vector of the objection functions, $G(\mathbf{x})$ is the feasible design space, $\mathbf{x}$ is a vector composition of the design variables, $M_{F}$ is the field coil mass and $M_{A}$ is the active mass.

Equation (5.6) represents Problem 1, while (5.7) represents Problem 2. In the first MDO prob-

\footnotetext{
21 Note that, because of a potential decrease in power density in the use of rare-earth-free materials in place of rare-earths, as well as the problematic torque ripple in FSMs, almost all of the optimisation problems declared in this dissertation have been focused on the minimisation of active mass or torque ripple, among others. As mentioned in Stipetic, Miebach and Zarko (2015) [88], the particular situation is a criteria of selecting the objective(s) for the design optimisation process.
} 
lem, the quest is to establish a relationship between the two objectives by minimising the $M_{F}$ and $M_{A}$ concurrently such that it satisfies the four performance constraints ascribed to it as indicated. Because of issues with hard constraint, which is occasioned by the highly restricted feasible search region in Problem 1, a second MDO problem is adjudicated such that it relaxes on the masses, but targets an optimum partnership between power factor and torque ripple performance, thereby limiting the constraints to only two parameters - output power and efficiency. In summary, the first problem majors on optimising the cost of the wind generator, while the second problem majors on optimising the cost of the SSCs.

The design variables, describing both dimensional and non-dimensional parameters, is given as

$$
\mathbf{x}=\left[\alpha, \mathrm{J}, \mathrm{J}_{F}, l_{s t}, D_{i n}, D_{s h}, h_{F}, b_{F}, b_{p r}, b_{s l s}, h_{y s}, h_{y r}, t_{0}\right],
$$

where $\mathrm{J}$ and $\mathrm{J}_{F}$ is the phase and field current density, $D_{s h}$ is the rotor shaft diameter, $h_{F}$ is the field core iron width and $t_{0}$ is a tapering factor for the rotor teeth defined as $b_{p r}^{\prime} / b_{p r}$. The rest of the parameters have either been previously defined in earlier chapters or in Appendix A1.2. However, in the case of accessing a quick reference of the general design variables applied in this dissertation, the section on technical nomenclature provided in the preliminary pages would suffice.

In the present circumstance, thirteen design variables have been nominated. The geometrical parameters considered for the MDO problems are illustrated in Appendix A1.2 as Fig. A1.5. For a realistic search domain during the MDO process, five boundary conditions which are necessary to harness the dimensional parameters of the WF-FSM are sequentially enumerated as follows:

$$
\begin{aligned}
& D_{\text {out }}-D_{\text {in }}^{(U)}>2 h_{y s}^{(U)}, \\
& D_{\text {out }}-D_{\text {in }}^{(U)}>2 h_{F}^{(U)}, \\
& \tau_{s}^{(L)}>b_{F}^{(U)}+b_{s l s}^{(U)}, \\
& D_{r o t}^{(L)}>D_{s h}^{(U)}+2 h_{y r}^{(U)}, \\
& \pi\left(D_{s h}^{(L)}+2 h_{y r}^{(L)}\right)>N_{r} b_{p r}^{(U)},
\end{aligned}
$$

where superscripts $L$ and $U$ are used to indicate the lower and upper limits of the respective parameter, $\tau_{s}$ is the stator pole pitch and $D_{r o t}$ is the rotor external diameter. Other parameters have their usual meanings. The boundary limits for the non-dimensional parameters are also imposed to the satisfaction of the design criteria.

The WF-FSM design is optimised to fit a frame size of stator outer diameter fixed at $600 \mathrm{~mm}$, based on estimation from (A1.4) in Appendix A2. Other parameters that were fixed during the MDO process include the slot fill factor for the phase and field slots, the number of turns for the phase and 
field slots, as well as the airgap length. Since NSGA-II algorithm is a stochastic process, the initialization of the design variables can be taken at random values which fall inside the boundary limits.

Two different optimisation runs are administered on Problem 1, the only difference being in the parameter settings as shown in Table 5.3. All the problems investigated were carried out within the same design space, using similar starting criteria for the design variables. To attain the final optimal solutions, a total 3750,4000 and 4000 design candidates are evaluated for the problems defined as $1 \mathrm{~A}, 1 \mathrm{~B}$ and 2 , respectively.

\subsubsection{Optimisation Results and Thoughts}

The converged Pareto optimal solutions are shown in Figs. 5.3, 5.4 and 5.5. The scatter plots (red markers) constitute the feasible search region, while the solid concentric circles (blue markers) are the Pareto optimal solutions, which constitute the Pareto optimal front (blue lines).

Although a lower population is debuted for Problem 1A compared to Problem 1B, an achievement of wider spread of solutions across the Pareto optimal front is witnessed in the latter because of the use of a smaller distribution index used, Deb et al: 2002 [107]. Both runs of Problem 1A and 1B show scanty populations due to constraints' violations at $19.3 \%$ and $12 \%$, respectively, in the feasible search region. However, the tradeoff which exists between the field coil mass and the active mass along the Pareto optimal front is clearly shown, with Problem 1B presenting the best outcome.

On the other hand, Fig. 5.5 shows that if the mass of the WF-FSM is not prioritized during the MDO process, the power factor can be significantly improved. As indicated along the Pareto optimal front in Fig. 5.5, significantly higher power factor are obtained at lower torque ripple values compared to the solutions of Problems 1A and 1B pictured in Figs. 5.3 and 5.4, respectively.

Table 5.3. NSGA-II parameters

\begin{tabular}{c|c|c|c}
\hline \multirow{2}{*}{ Parameter } & \multicolumn{3}{|c}{ Problems } \\
\cline { 2 - 4 } & 1A & 1B & 2 \\
\hline Mutation probability, $P_{m}$ & \multicolumn{3}{|c}{0.077} \\
Crossover probability, $P_{c}$ & 0.95 & 0.9 & 0.9 \\
Mutation distribution index, $\eta_{\mathrm{m}}$ & 20 & 10 & 10 \\
Crossover distribution index, $\eta_{\mathrm{c}}$ & 20 & 20 & 20 \\
Populations & 25 & 40 & 40 \\
Iterations & 150 & 100 & 100 \\
\hline
\end{tabular}




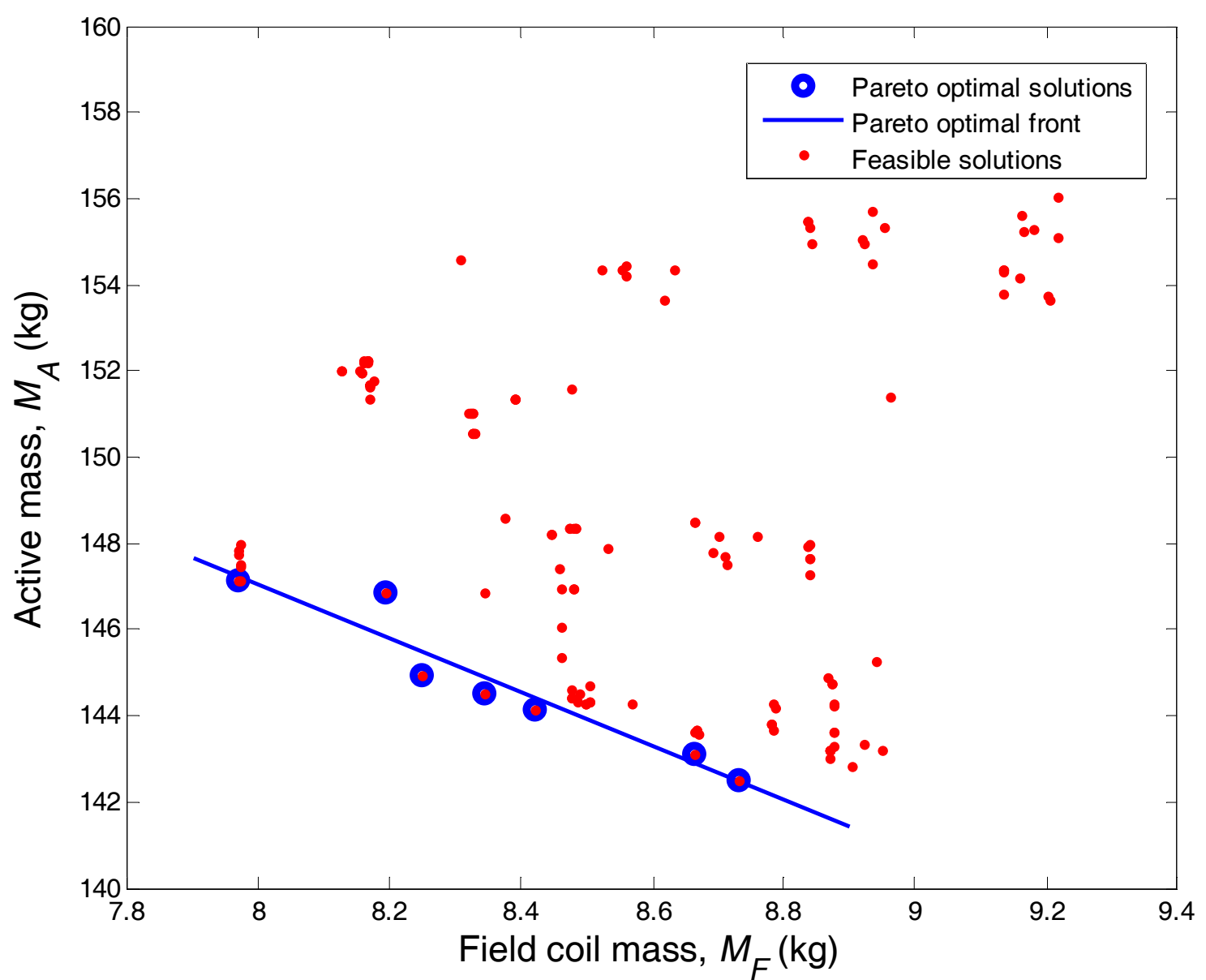

Fig. 5.3. Obtained Pareto optimal front for Problem 1A.

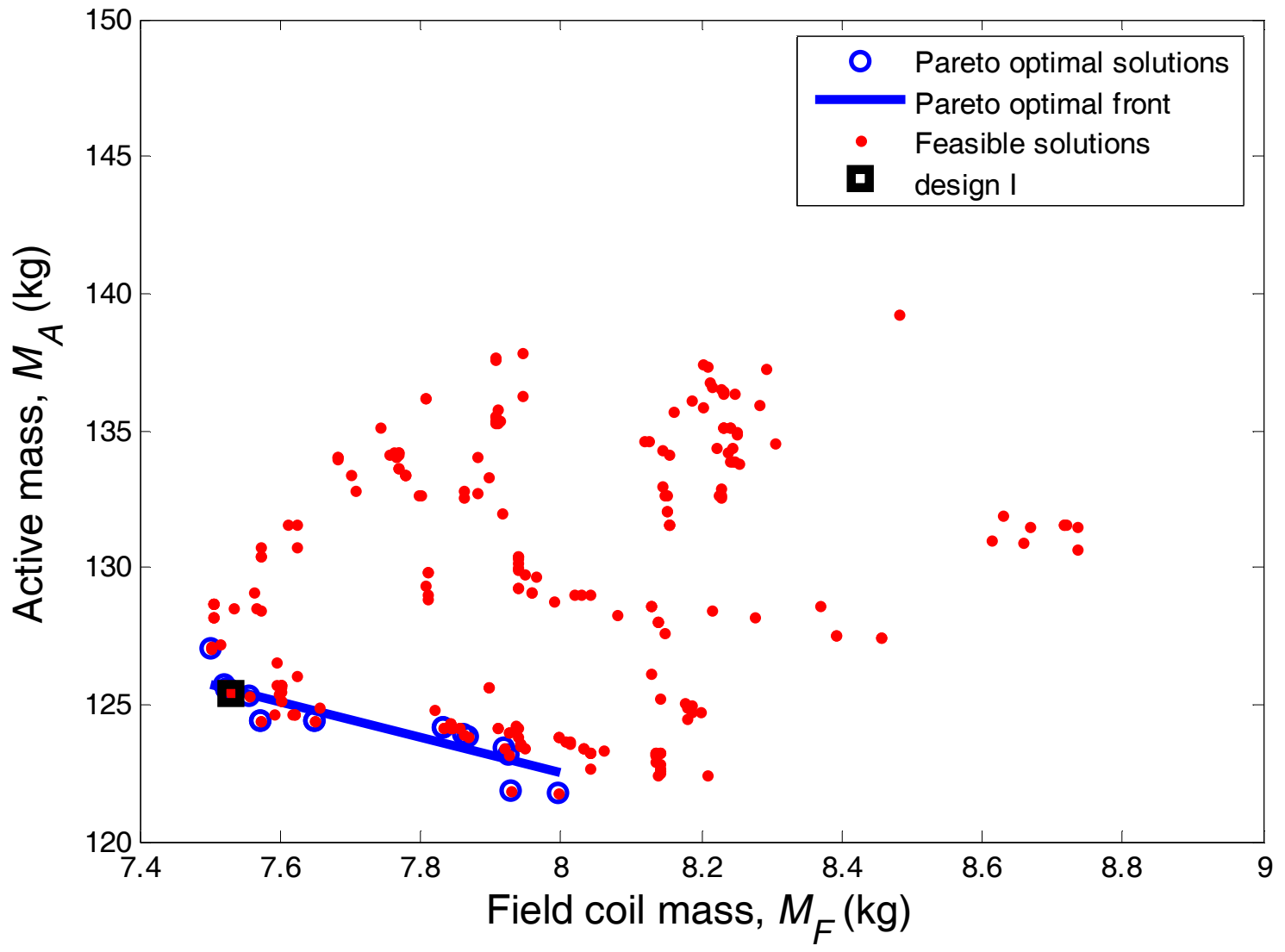

Fig. 5.4. Obtained Pareto optimal front for Problem 1B. 


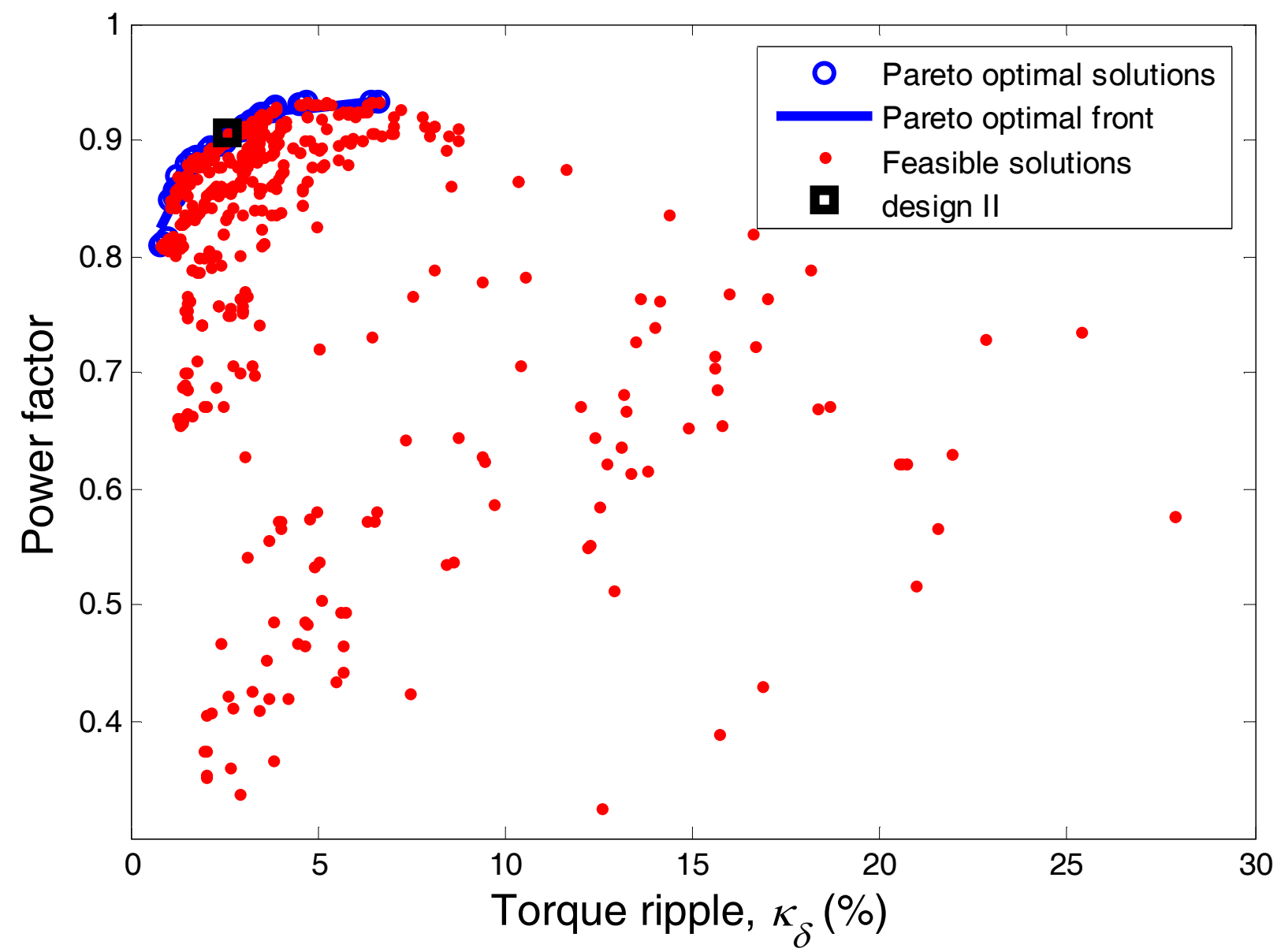

Fig. 5.5. Obtained Pareto optimal front for Problem 2.

To better appreciate the WF-FSM performance, two benchmarks are made for further evaluation and comparison. As indicated by the points (black square markers) in Figs. 5.4 and 5.5, independent selections - designs I and II — are randomly made with emphasis on the "optimum" operating points for the proposed wind generator drivetrain. In Table 5.4, the selected designs are compared with regards to satisfying the optimal performance criteria, with both the FEA-based analytically formulated results $\left(\Upsilon_{1}\right)$, as well as the actual FEA $\left(\Upsilon_{2}\right)$ shown. The following conclusions are made:

I. Although design II witnessed higher output power compared to design I, its torque density is smaller by $23.3 \%$ because the component mass is not minimised.

II. Although the active mass of design I decreased by $60.4 \%$ compared to design II, there is no significant advantage in terms of efficiency. Besides, the field to active mass ratio when considered is approximately the same in both optimal design candidates.

III. The power factor in design II compared to design I is superior by $12.5 \%$. In the same token, the torque ripple is significantly lower in the former $(-71.8 \%)$.

IV. Similar to what obtained in Zhang, Ionel and Demerdash (2016) [89] for IPM machines, lower power factor as observed in design I is because of the main objectives which focused on 
minimum mass. Thus, no clear advantage is achieved in both selected candidates in terms of saliency ratio, seen to be identical.

V. Lastly, for both designs, the major limitation between using actual FEA results to using those calculated from analytic formulations as derived from the FEA, is expressed in terms of the torque ripple. This is due to the fact that the torque expression adopted, which is based on (2.8) in section 2.2 in Chapter 2, does not explicitly capture the actual undulation of the onload airgap field distribution as is the case in the process based purely on FEA.

In summary, design I presents an optimum solution for minimum size and cost of the proposed wind generator, within the respected performance limits. However, if the cost of the generator is not prioritised during the design optimisation stage, then another optimum design can be attained whereby the performance of the generator may result in smaller kVA ratings in respect to cost savings for the SSC. To explain this phenomenon, it is observed that in the case of design I, a higher level of restraint is expected with regards to the electromagnetic performance because of the greater number of performance constraints required to achieve minimum generator mass, vis-à-vis the manufacturing costs.

\subsection{3-D FEA Evaluation}

Insofar as this study is based on 2-D magnetostatic FEA solutions aided by analytical formulations, this subsection discusses additional evaluations performed in 3-D transient FEA simulations.

Table 5.4. Comparison of optimal performance indices

\begin{tabular}{c|c|c|c|c|c}
\hline \multirow{2}{*}{ Quantity } & \multicolumn{2}{|c|}{ design I } & \multicolumn{2}{c|}{ design II } & \multirow{2}{*}{ Deviation $^{22}$} \\
\cline { 2 - 5 } & $\boldsymbol{\Upsilon}_{\mathbf{1}}$ & $\boldsymbol{\Upsilon}_{\mathbf{2}}$ & $\boldsymbol{\Upsilon}_{\mathbf{1}}$ & $\boldsymbol{\Upsilon}_{\mathbf{2}}$ & \\
\hline \hline$\tau_{e}(\mathrm{Nm})$ & 258.76 & 258.71 & 318.99 & 318.98 & $23.27 \%$ \\
$\boldsymbol{\kappa}_{\delta}(\%)$ & 9.11 & 34.42 & 2.56 & 47.76 & $-71.89 \%$ \\
$\mathrm{P}_{\mathrm{out}}(\mathrm{kW})$ & 10.04 & 10.01 & 12.22 & 12.22 & $21.71 \%$ \\
$\eta(\%)$ & 88.59 & 88.59 & 87.92 & 87.92 & $-0.75 \%$ \\
$\mathrm{PF}$ & 0.8 & 0.78 & 0.9 & 0.9 & $12.50 \%$ \\
$M_{F}(\mathrm{~kg})$ & 7.53 & 11.73 & $55.77 \%$ \\
$M_{A}(\mathrm{~kg})$ & 125.43 & 201.19 & $60.40 \%$ \\
$\tau_{e} / M_{A}(\mathrm{Nm} / \mathrm{kg})$ & 2.06 & 1.58 & $-23.30 \%$ \\
$\mathrm{~J}\left(\mathrm{~A} / \mathrm{mm}^{2}\right)$ & 2.70 & 2.22 & $-17.77 \%$ \\
$\mathrm{~J}_{F}\left(\mathrm{~A} / \mathrm{mm}^{2}\right)$ & 4.98 & 4.98 & $0 \%$ \\
$X_{d}(\Omega)$ & 40.41 & 82.98 & $105.34 \%$ \\
$X_{q}(\Omega)$ & 39.24 & 81.18 & $106.88 \%$ \\
$S^{23}$ & 0.98 & 0.98 & $0 \%$ \\
\hline
\end{tabular}

\footnotetext{
22 The deviations are referenced to solutions in $\Upsilon_{1}$.
} 
To this end, Problem 2 is repeated, but this time the torque, together with the torque ripple, is exclusively evaluated by 2-D FEA, while other performance parameters (power factor and efficiency) still follow from the FEA cum analytic formulations. The only alteration done in this case is in respect of the NSGA-II parameter settings earlier shown in Table 5.3, by which the number of iterations is increased to 125 , resulting in a total of 5000 design candidates evaluated.

The obtained Pareto front is shown in Fig. 5.6. As can be seen, the trend is consistent with previous observation in Fig. 5.5. The fact that, in Fig. 5.6 compared to Fig. 5.5, the Pareto front tends to curve further away from the axes peripheral to it, and stretches towards both axes in the feasible design space, beyond other factors, is an indication that the torque ripple estimation was underrated in the latter case. Based on the new solutions, design III is selected as highlighted in Fig. 5.6.

Also highlighted in Fig. 5.6 are the optimal aspect $\left(\kappa_{L}\right)$ and split $\left(\Lambda_{0}\right)$ ratios for design III, with the two extreme Pareto optimal solutions highlighted by points " $X$ " and " $Y$ ". Not much difference is observed in these values when compared because the stator outer diameter is kept constant throughout the design optimisation process.

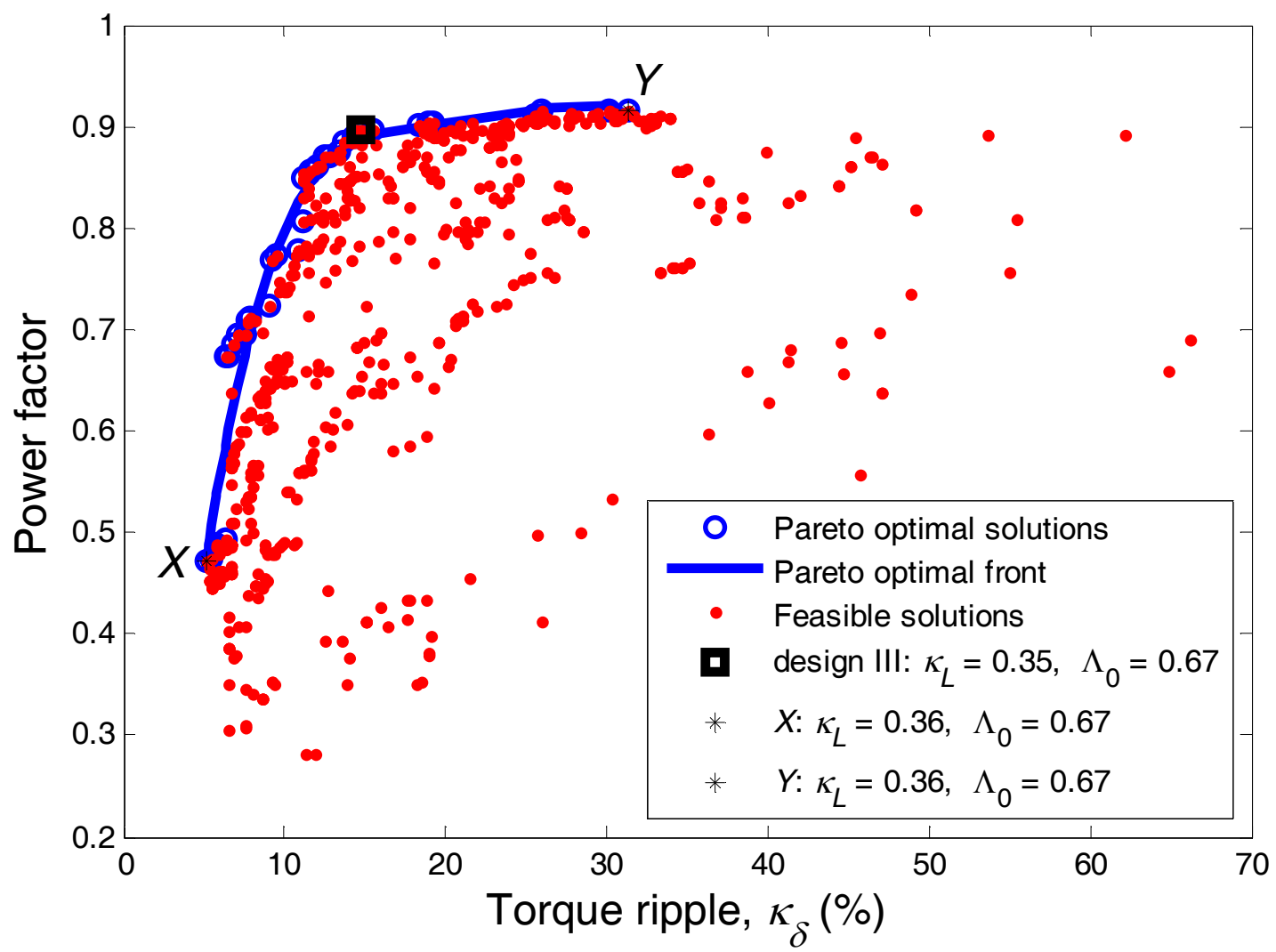

Fig. 5.6. Obtained Pareto optimal front for Problem 2, showing the torque ripple evaluated from purely 2-D FEA torque output. 


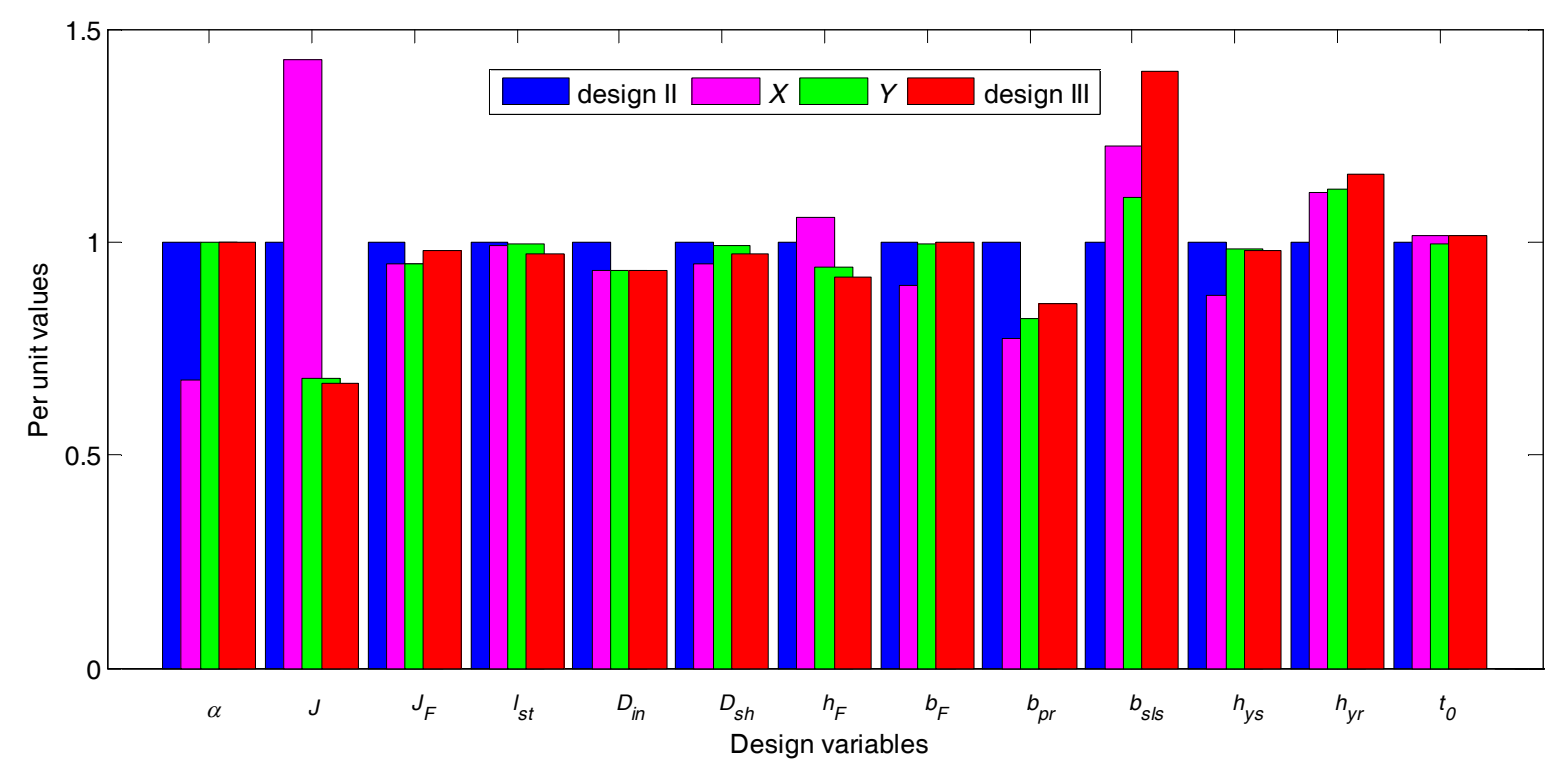

Fig. 5.7. Per unit values of four optimum WF-FSMs selected from Problem 2 along the Pareto front.

In Fig. 5.7, the highlighted design points in Fig. 5.6 are contrasted in order to emphasise key design variables affected by the technique adopted for design II. It can be seen that the design parameters heavily affected in design III are primarily related to the rotor or airgap such as $b_{p r}, b_{s l s}$ and $h_{y r}$, with the exception of $h_{F}$ and $\mathrm{J}$ which are mainly stator parameters. This is due to slot effects and a double salient structure, critical to the torque ripple. Unlike what obtained when $\kappa_{L}$ and $\Lambda_{0}$ are compared for the design points " $X$ " and " $Y$ ", the design parameters, $J$ and $\alpha$, among others, significantly influence the extreme outcomes. Considering the FSM generator phasor diagram shown in Fig. 2.1 in section 2.2 in Chapter 2, the reason for suppressed power factor at point " $X$ " becomes plausible.

To further emphasize the cost implication, $M_{F}$ and $M_{A}$ for design III is $14.85 \mathrm{~kg}$ and $198.54 \mathrm{~kg}$, respectively. By comparing values of design III to those of design II (Table 5.4), the ratio $M_{F} / M_{A}$ yield $7.47 \%$ and $5.83 \%$, respectively. The disparity occurs because the technique used in realising design III seriously impedes the power factor (compare Fig. 5.5 and Fig. 5.6), leading to increase in the field current in design III, justified by the decrements in $\mathrm{J}_{F}$ and $h_{F}$ as shown in Fig. 5.7.

Based on design requirements set forth in Table 5.1, the obtained 2-D static FEA results for design III are compared in 3-D transient FEA. The mesh configurations and magnetic field/flux lines are shown in Fig. 5.8. The THD rates measured in the 2-D FEA and 3-D FEA induced terminal voltages, as compared in Fig. 5.9, are not only low but show good confidence.

Table 5.5 which displays the comparative performance also shows good agreement, except for the $\kappa_{\delta}$, which is likely afflicted by the coarse meshing used in the 3-D FEA modelling. Hence, the higher values recorded in 3-D FEA for $\tau_{e}$ cum $\mathrm{P}_{\text {out }}$ is due to $\tau_{e}$ and $\kappa_{\delta}$ being evaluated directly in 2-D FEA, while other performance parameters are evaluated as previously in the FEA-analytical method. 


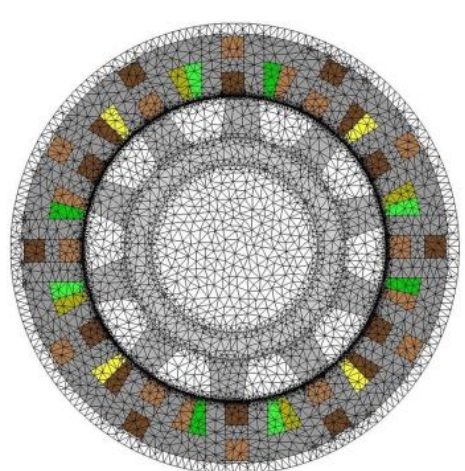

(a)

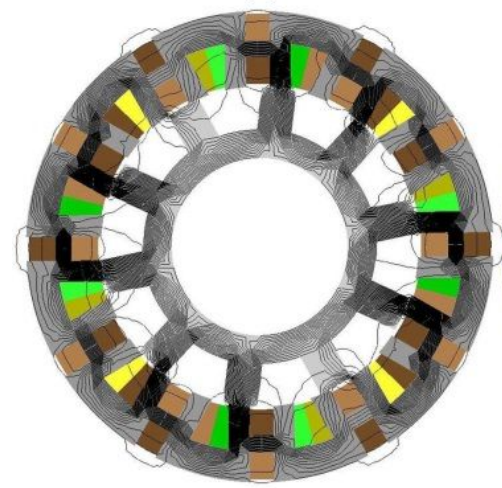

(c)

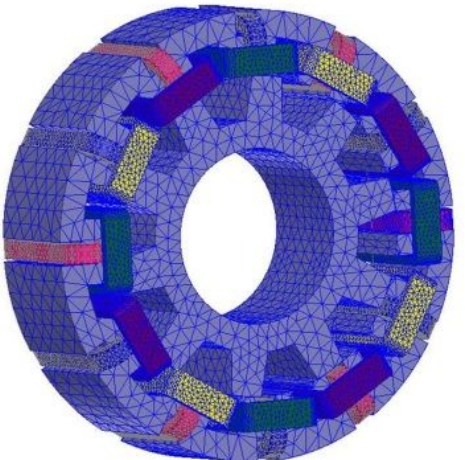

(b)

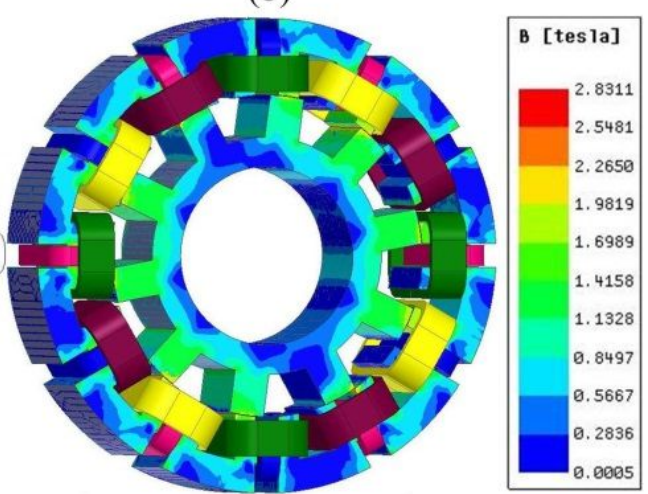

(d)

Fig. 5.8. FEA display of: (a) finer 2-D mesh structures, (b) coarse 3-D mesh structures, (c) magnetic fields in 2-D static solution, and (d) flux density surface map in 3-D transient solution.
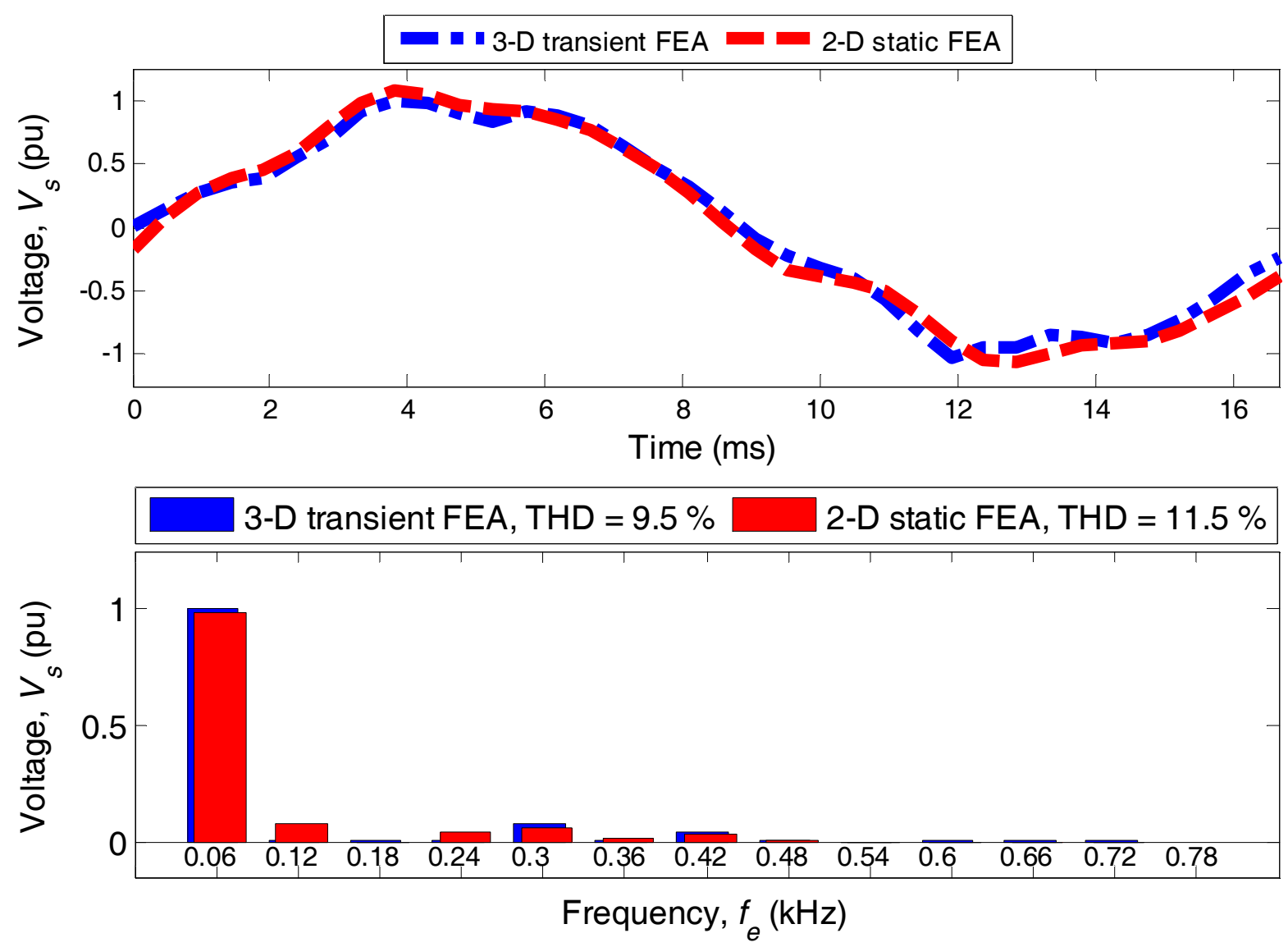

Fig. 5.9. Nominal induced phase voltage waveforms at $360 \mathrm{r} / \mathrm{min}$ and the resulting frequency spectral harmonics in 2-D static and 3-D transient FEA. 
Table 5.5. Validation of optimal performance indices based on Design III

\begin{tabular}{c|c|c|c}
\hline \multirow{2}{*}{ Performance quantity } & \multicolumn{2}{|c|}{ FEA } & \multirow{2}{*}{ Deviation } \\
\cline { 2 - 3 } & 2-D static & 3-D transient & \\
\hline Electromagnetic torque, $\tau_{e}(\mathrm{Nm})$ & 321.43 & 329.87 & $2.55 \%$ \\
Torque ripple, $\kappa_{\delta}(\%)$ & 14.80 & 25.58 & $42.12 \%$ \\
Output power, $\mathrm{P}_{\text {out }}(\mathrm{kW})$ & 12.89 & 13.07 & $1.37 \%$ \\
Power factor, $\mathrm{PF}$ & 0.89 & 0.87 & $-2.29 \%$ \\
Copper loss, $P_{C u}(\mathrm{~kW})$ & 1.329 & 1.326 & $-0.22 \%$ \\
Core loss, $P_{\text {Core }}(\mathrm{kW})$ & 0.464 & 0.303 & $-53.13 \%$ \\
Efficiency, $\eta(\%)$ & 87.78 & 88.67 & $-1.00 \%$ \\
\hline
\end{tabular}

The higher $\eta$ recorded in 3-D FEA as opposed to 2-D FEA, is because of the remarkable difference in $P_{\text {Core }}$ as shown in Table 5.5. In particular, the discrepancy in $P_{\text {Core }}$ is due to the lower rotor core losses observed in 3-D transient FEA compared to 2-D static FEA. In 3-D FEA, this characteristic is as a result of the non-corresponding effect of the stator current on the rotor iron loss as reported in Chen et al (2010) [64].

\subsection{Chapter Summary}

In this chapter, constrained MDO has been administered on the 12/10 WF-FSM topology, designed for geared MS wind generator drives. Using 2-D FEA-based formulations coupled to a non-gradient optimisation algorithm (NSGA-II), the production of a Pareto optimal solution set is guaranteed. Two problems were defined for the MDO process, such as Problem 1: to minimise both the field copper and active mass; and Problem 2: to minimise torque ripple while maximising power factor. Problem 1 having two separate runs, quickly reveals the tradeoff existing between the field coil mass and the active mass along the Pareto optimal front, whereas Problem 2 shows, for the first time, that to achieve high power factor in WF-FSMs, a corresponding high torque ripple circumstance results. In other words, "to improve the power factor of the WF-FSM, a compromise lies in the torque ripple".

Thus, by isolating two optimal design candidates, i.e., one from each problem, the importance in approaching the MDO problem from either a manufacturing or performance constraints' viewpoint becomes obvious. When restricted to the same design environment, the WF-FSM performance is constrained if the MDO problem is solely pursued in terms of minimising the cost of materialsmanufacturing. On the other hand, approaching the MDO problem from a solely performance viewpoint (optimum power factor and torque ripple) could result in beneficial handouts to the overall wind generator drivetrain such as diminishing the kVA rating, viz., the cost of the SSCs.

Lastly, a comparison between 2-D static FEA and 3-D transient FEA is propagated, which 
shows good agreement. But a potential limitation is observed whereby the use of simplified analytical formulations from the dq flux linkages in static 2-D FEA, in place of direct FEA calculations, underestimates the torque ripple calculations. 


\section{ChAPTER SiX}

\section{Design Characteristics and Potentials of Rare- EARTH-Free FSMS fOr LARGE-SCALE Wind GeNERATOR DRIVES}

In this chapter, the design optimisation of rare-earth-free FSMs, viz., ferrite PMs and wound-fields, is embarked upon for geared MS wind generator applications, for both small-scale $(\mathrm{kW})$ and largescale (MW) power levels. Again, a distinction between this study and that conceived in Chapter 4 is that both the ferrite PM-FSM and WF-FSM are considered for their 12/10 and 12/14 topologies. In addition, a different MDO approach from that promulgated in Chapters 4 and 5 is undertaken. Thus, to the best of the researcher's knowledge, this is the first time both WF-FSM variants considered, are being nominated for any such design characterisation. The main focus is on the optimum performance of these machines in place of more expensive rare-earth PM designs, as well as their suitable candidature for the proposed wind generator drivetrain. In addition, the comparison between the $12 / 10$ and 12/14 machines are further elucidated in terms of their scalable operating power level characteristics. Therefore, the inquiry in this chapter is a follow-up on findings earlier obtained in Chapter 3, which indicated the potentials of rare-earth-free FSMs based on improved performance characteristics of rare-earth PM-FSMs in the large-scale power levels compared to small-scale designs.

\subsection{Introduction}

As it stands, FSMs are increasingly gaining popularity, not only in design and analyses, but in a wide variety of applications. Among the numerous topologies in existence today and currently under development, the researcher has shown that the simple radial-flux topology is proven as the most interesting topology, among which are the three-phase 12/10 and 12/14 designs as earlier illustrated in Fig. 3.1 in section 3.2 in Chapter 3.

Also, as earlier mentioned, the 12/10 design was originally proposed as a ferrite PM-FSM by Hoang, Ben Ahmed and Lucidarme (1997) [58], whereas Chen et al (2009) [82] are to be credited for conceiving the 12/14 machine. Already, a number of studies have considered the design and/or analyses of these two machine variants based PM field excitation sources, e.g., Amara et al (2005) [54], Zhu et al (2008) [62], Chen and Zhu (2010) [65], Fasolo, Alberti and Bianchi (2014) [69] and 
Raminosoa et al (2015) [71], to mention a few. Although ferrite PMs have been routinely used in such studies, but due to their lower energy potentials, they are not so much popular like those designed with rare-earth PMs.

As for wound-fields, the first people who conceptualised the 12/10 and 12/14 topologies were Tang et al (2012) [67], with comparable torque capabilities to PM-FSMs when so designed. But, it has to be said that they only delved into analyzing the 12/14 WF-FSM, and abandoned the 12/10 topology. With all modesty, the researcher happens to have been the first person who seriously considered the design and analyses of the 12/10 WF-FSM after their work.

To this end, the present study is the first time that both WF-FSM topologies are conclusively being characterised together with their ferrite PM-FSM counterparts for $10 \mathrm{~kW}$ and $3 \mathrm{MW}$ wind generator applications. Note that, the attempt made earlier by the researcher in Akuru and Kamper (2016) [111], only focused on $1.5 \mathrm{~kW}$ power of their 12/10 machines.

The main motivation for the current study is that other researchers have exposed the potentials of these machines in automotive drive applications, Fasolo, Alberti and Bianchi: 2014 [69] and Raminosoa et al: 2015 [71], with the exception of what the researcher has been doing on wind generator applications. In addition, note that it is desired for wind generator designs, like in most electrical machine systems, for the cost of the total set-up to be minimised as much as possible. One way by which this can be conquered is to use abundant and less expensive raw materials for the design of such wind generators, e.g., wound-fields and ferrite PMs. Apparently, rare-earth PM which has been very popular with PM-FSMs cannot be considered a cheaper option because of three major reasons: 1) PM-FSM are PM-intensive machines adopting so-called flux focusing principles, 2) rare-earth PM are highly priced compared to ferrite PMs, and 3) the market structure of rare-earth PMs is a monopoly.

But as earlier revealed in Chapter 4, ferrite PM-FSM can result in $35 \%$ cost savings in material components over similarly designed rare-earth PM machines at $10 \mathrm{~kW}$ power. Although the same cost advantage may not be claimed for WF-FSMs designed at the same power level, but it remains to be seen what happens when the power level dramatically increased. Moreover, it should not be forgotten that with WF-FSMs, what is lost in terms of cost of the materials may be gained in terms of absence of demagnetisation risks, as well as the potentials for regulating the generator power output in tandem with the fluctuating wind energy resource for optimum power delivery. Lastly, it must be added that unlike conventional wound-field machines, WF-FSMs are brushless machines, whereby they require no brushes and slip rings for their operation.

Therefore, with the right power range, a proper account on the effects of scaling the proposed 
wind generators can be made available, especially to provide insight for utility-scale purposes. Hence, in this chapter, the optimum design characteristics of both the 12/10 and 12/14 machines, implemented as rare-earth-free configurations, is undertaken by means of 2-D static finite element analyses (FEA), for small-scale $(10 \mathrm{~kW})$ and large-scale $(3 \mathrm{MW})$ wind generator drives. As a reminder, the proposed wind generator drive is based on the geared medium-speed (MS) drivetrain, which has been declared as the best in terms of performance to cost index for different generator topologies, Cao, Xie and Tan: 2012 [4], Schmidt and Vath: 2012 [20] and Bang et al: 2008 [29]. An illustration of the proposed geared MS drivetrain system, which has been serially referred to in this dissertation is as pictured in Fig. 1.12 in subsection 1.3.3 in Chapter 1. But, it has to be reiterated that the focus of the study is still on the electromagnetic design and analyses of the wind generator itself in respect to other drivetrain components.

In the following sections, a description of the design optimisation method used for the study is briefly revisited, after which the optimisation results are discussed for the designated power levels. Thereafter, some selected optimal design samples are compared ab intra and inter alios to other conventional wind generator topologies analysed in the literature, within the same drivetrain. Lastly, 3-D models of some benchmark designs are constructed and proven in transient FEA simulations.

\subsection{Design Optimisation Process}

It is commonly agreed that in the design optimisation of electrical machines, a constrained multiobjective problem is the preferred choice, due to many conflicting design parameters competing for relevance, El-Wakeel: 2004 [129]. However, the contention is still on the preferred optimisation algorithm - be it deterministic or stochastic (metaheuristic). Whereas the former is fast, cheap and follows after the gradient method, it is limited because it depends on a predefined starting point which may lead the optimal solution astray in the event that many local minima/maxima exist inside the search arena. Instead, stochastic algorithms which are inauspiciously time and cost intensive are more flexible and attractive to tackle global optimisation problems. Although there is equally no guarantee that a firm optimal solution can be obtained with such metaheuristics because of their non-gradient solutions, however there is a high chance that the final solution lies very, very close.

Thus, the NSGA-II is a typically robust metaheuristic initially developed by Deb et al: 2002 [107], which has been basically adopted in this dissertation. A major highpoint in the use of NSGAII for electrical machine design optimisation is the fact that it is able to generate a Pareto optimal solution set when invited to multi-objective problems.

To this end, NSGA-II linked by FEA evaluations is being escalated further in the design anal- 
yses of the proposed rare-earth-free FSMs, based on a constrained MDO approach. Besides, the optimisation suite, VisualDOC (2017) [93], where the NSGA-II functionality is readily accessible, is used along with the robust and versatile 2-D static FEA open-source package-SEMFEM [130]. Both simulation tools are interfaced as shown in Fig. 1.13 in section 1.5 in Chapter 1. The workflow in Fig. 6.1, not previously shown in this dissertation, shows how each model is conceived and solved in SEMFEM, and then tossed back-and-forth through the optimiser until some convergence is obtained.

The optimisation problem to be tackled, which requires the minimization of two objectives and the declaration of three design constraints, is set up as follows

$$
\boldsymbol{F}(\bar{x})=\left[\begin{array}{c}
M_{A} \\
\kappa_{\delta}
\end{array}\right] ; \boldsymbol{G}(\bar{x})=\left[\begin{array}{c}
\mathrm{PF} \\
\mathrm{P}_{\text {out }} \\
\eta
\end{array}\right] ;
$$

with active mass $\left(M_{A}\right)$, torque ripple $\left(\kappa_{\delta}\right)$, output power $\left(\mathrm{P}_{\text {out }}\right)$, efficiency $(\eta)$ and power factor $(\mathrm{PF})$, explicitly defined in section 2.2 in Chapter 2. Note that, $M_{P M}$ as defined in (2.18) is changed to $M_{F}$ when the considering a WF-FSM, as well as $P_{C u}$ in (2.15) which becomes the total copper loss of both the wound-fields and three-phase coils. In the 2-D FEA process, the end-windings have been modelled accordingly as described already in subsection 5.3 .2 of the preceding chapter.

A total of 12 and 13 design parameters $(\bar{x})$ are varied for the ferrite PM-FSM at $10 \mathrm{~kW}$ and 3 MW, respectively. On the other hand, 14 design variables are registered for the WF-FSM at both 10 $\mathrm{kW}$ and $3 \mathrm{MW}$ power levels. The total design variables summoned at any time for the optimisation procedure is comprised in the array

$$
\bar{x}=\left[\alpha, g, \mathrm{~J}, \mathrm{~J}_{F}, l_{s t}, D_{o u t}, D_{i n}, D_{s h}, b_{p m}, b_{F}, b_{p r}, b_{s l s}, h_{y s}, h_{F}, h_{y r}, t_{0}\right],
$$

where $g$ is the airgap length, $\mathrm{J}$ and $\mathrm{J}_{F}$ is the phase and field current density, $l_{s t}$ is the stack length, $D_{\text {out }}$ is the stator outer diameter, $D_{i n}$ is the stator interior diameter, $D_{s h}$ is the rotor shaft diameter, $b_{p m}$ is the PM length only for the ferrite PM-FSMs, $b_{F}$ is the field core iron length only for the WFFSMs, $b_{p r}$ is the rotor pole width, $b_{s l s}$ is the stator slot opening width, $h_{y s}$ is the stator yoke height, $h_{F}$ is the field core iron width only for the WF-FSMs, $h_{y r}$ is the rotor yoke height and $t_{0}$ is a tapering factor used to temper on the rotor teeth.

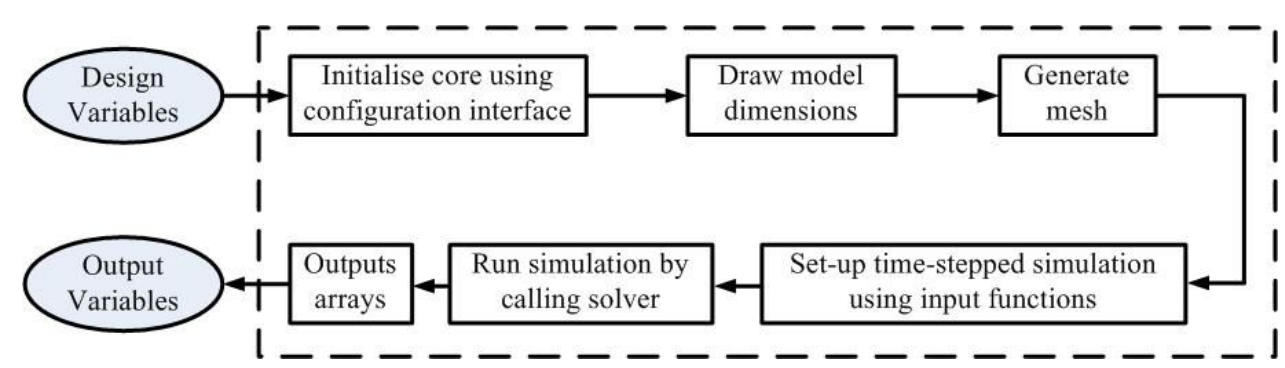

Fig. 6.1. Typical workflow processed in 2-D FEA (SEMFEM) simulation. 
Due to the high sensitivity of the WF-FSM designs to copper loss which limits the efficiency requirements, their copper windings were estimated at operating temperatures of $80{ }^{\circ} \mathrm{C}$ in order to increase the design reliability, whereas the copper windings in the ferrite PM-FSMs were maintained at room temperature values $\left(25^{\circ} \mathrm{C}\right)$. In addition, the efficiency and output power specifications are administered differently depending on either the power level or the type of field source used. For instance, the power limits for all the $\mathrm{kW}$ designs was registered to at least $10 \mathrm{~kW}$, while that of the MW designs to at least $3 \mathrm{MW}$. The efficiency of all $3 \mathrm{MW}$ designs was set to at least $97 \%$, while those of the $10 \mathrm{~kW}$ ferrite PM-FSMs and WF-FSMs were set to at least $90 \%$ and $89 \%$, respectively.

In NSGA-II, the primary fitness is evaluated based on domination level while the secondary fitness is evaluated based on the diversity of the solution in its domination level, each fitness level represented as user-tunable parameters. Further descriptions on these parameters have been provided in Deb et al: 2002 [107] and VisualDOC (2017) [93]. Thus, for the different simulations undertaken in the present study, it was necessary to tune the NSGA-II operators as indicated in Table 6.1.The difference observed in the mutation probability is simply because it is taken as the inverse of the number of design variables considered for each MDO problem category indicated. For each problem, the upper and lower boundaries of the prescribed design parameters are also fine-tuned in order to ensure geometrical feasibility. The results obtained after each simulation are presented and discussed in the subsequent section.

\subsection{Design Optimisation Results, Observations and Discussions}

The optimisation results for the $12 / 10$ and $12 / 14$ machines at $10 \mathrm{~kW}$ and $3 \mathrm{MW}$ are displayed in Figs. 6.2-6.5. At $10 \mathrm{~kW}$ power, the optimal candidates of the 12/14 ferrite PM-FSM yield slightly lower active mass and torque ripple values compared to optimal candidates of the 12/10 ferrite PM-FSM as shown in Fig. 6.2. In Fig. 6.4, a similar outcome is represented for the ferrite machines at 3 MW power levels, whereby a decrease in the active mass is more significantly represented in the $12 / 14$ optimal candidates compared to the 12/10 optimal designs.

On the other hand, Fig. 6.3 indicates that the $10 \mathrm{~kW}$ WF-FSM optimal designs are not able to produce lower torque ripple values usually expected of the 12/14 machines when compared to their $12 / 10$ counterparts. Rather, it is observed that the $12 / 10$ machines can achieve up to $50 \%$ deficit beyond the least torque ripple values realisable in the 12/14 machines, but with slightly lower active mass in the former. As observed in Fig. 6.5, this tendency is further escalated to more than $55 \%$ deficit of the lowest torque ripple feasible in the 12/10 machines compared to the 12/14 machines at 3 MW power levels. This discrepancy is because the efficiency requirements in the WF-FSM, which is 
originally limited by the presence of the wound-field coils, but further suppressed by the presence of higher core losses in the 12/14 machines compared to the 12/10 machines.

Considering the ferrite PM-FSM and WF-FSM at $10 \mathrm{~kW}$ power levels (Figs. 6.2 and 6.3), it is noteworthy to see that the best optimum designs (lowest torque ripple and active mass) were obtained for both the 12/10 and 12/14 machines. However, the same cannot be said for the $3 \mathrm{MW}$ design (Figs. 6.4 and 6.5), whereby the lowest torque ripple is possible for the ferrite PM-FSMs by up to $49 \%$ (in the $12 / 14$ machines) and also the WF-FSMs by up to $30 \%$ (in the $12 / 10$ machines).

Table 6.1. NSGA-II Parameters

\begin{tabular}{c|c|c|c|c}
\hline \multirow{2}{*}{ Parameters } & \multicolumn{2}{|c|}{ Ferrite PM-FSM } & \multicolumn{2}{c}{ WF-FSM } \\
\cline { 2 - 5 } & $\mathbf{1 0} \mathbf{~ k W}$ & $\mathbf{3 ~ M W}$ & $\mathbf{1 0} \mathbf{~ k W}$ & $\mathbf{3 ~ M W}$ \\
\hline \hline Mutation probability & 0.083 & 0.076 & 0.071 & 0.071 \\
Crossover probability & 0.9 & 0.9 & 0.9 & 0.9 \\
Mutation distribution index & 10 & 10 & 10 & 10 \\
Crossover distribution index & 20 & 20 & 20 & 20 \\
Population size & 20 & 25 & 20 & 20 \\
Iterations & 100 & 100 & 100 & 100 \\
\hline
\end{tabular}

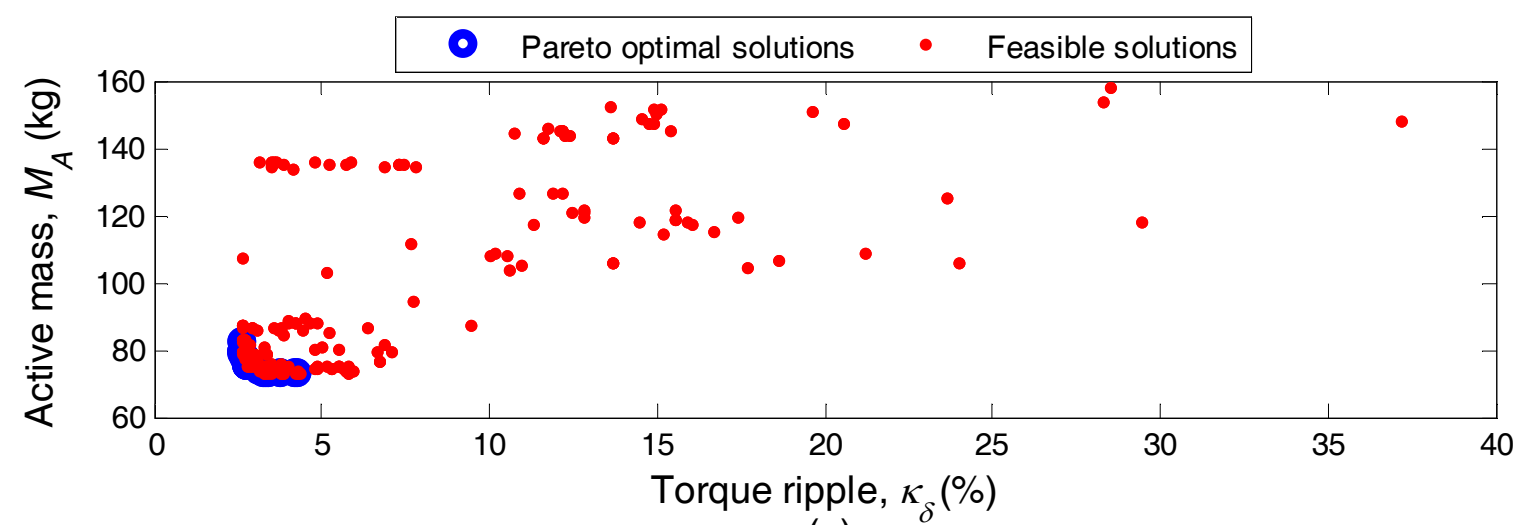

(a)

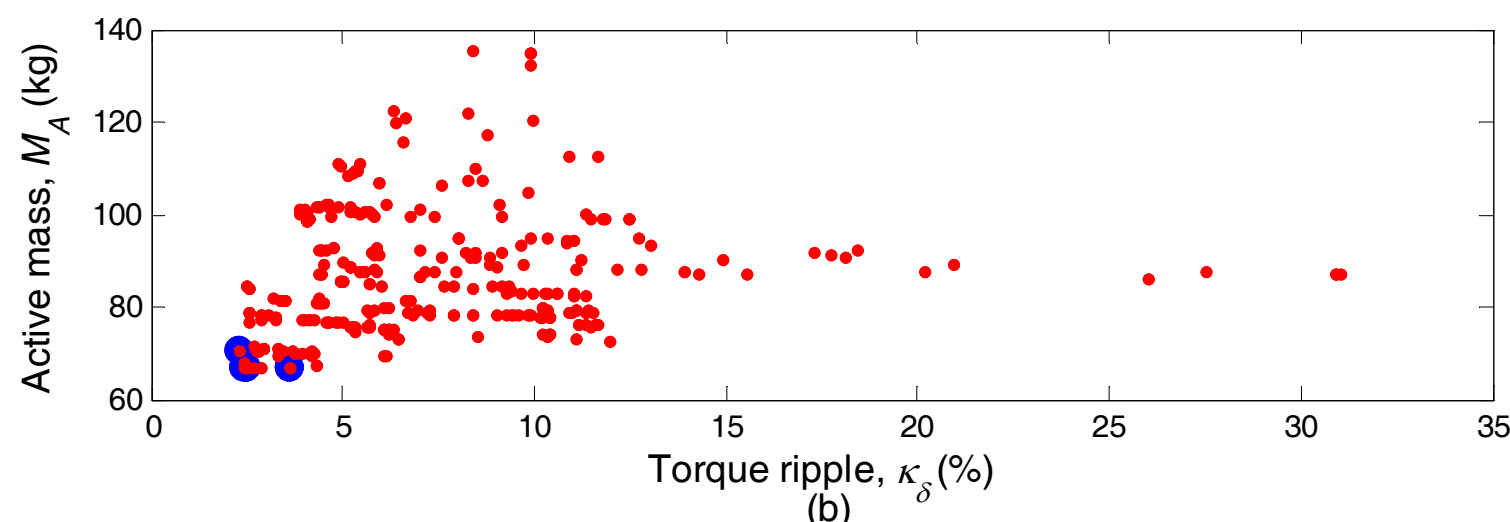

Fig. 6.2. Pareto optimal fronts for different $10 \mathrm{~kW}$ ferrite PM-FSMs: (a) 12/10, and (b) 12/14. 


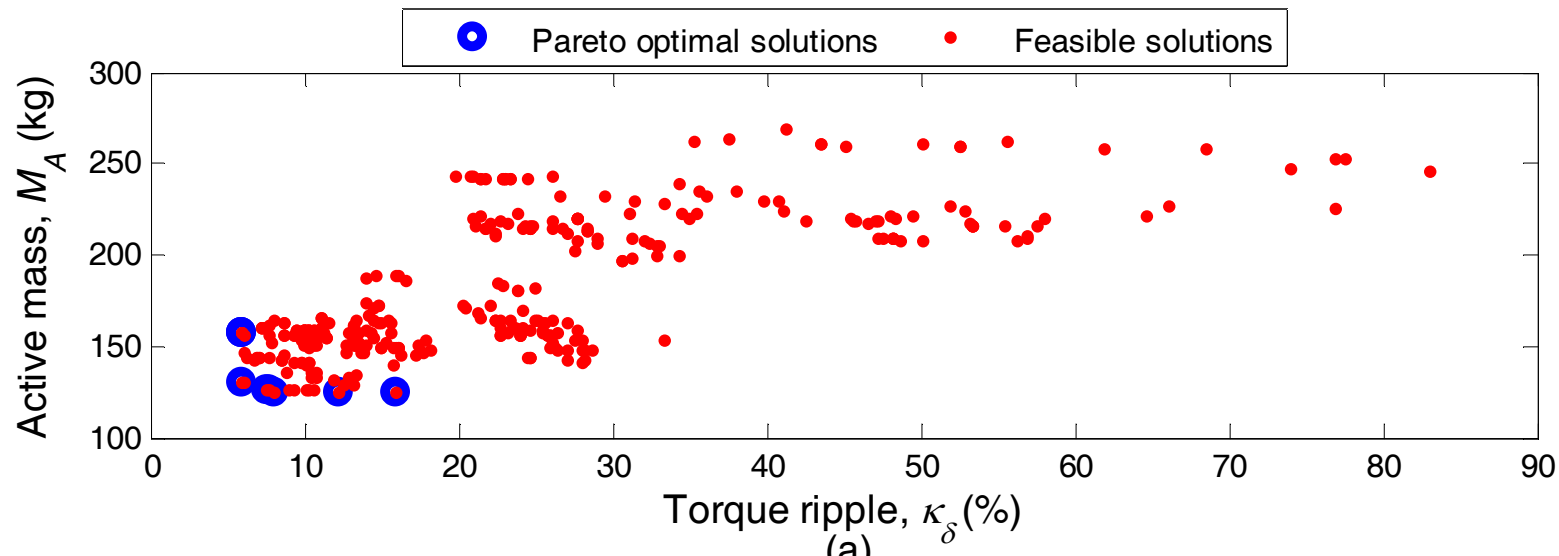

(a)

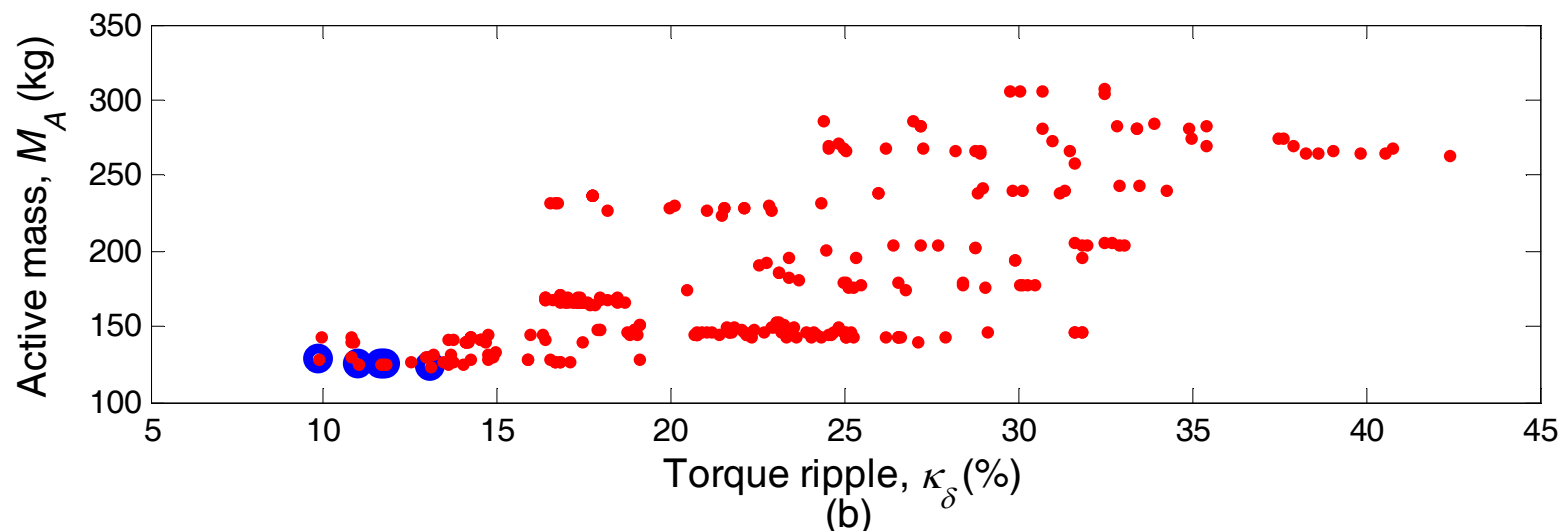

Fig. 6.3. Pareto optimal fronts for different $10 \mathrm{~kW}$ WF-FSMs: (a) 12/10, and (b) 12/14.
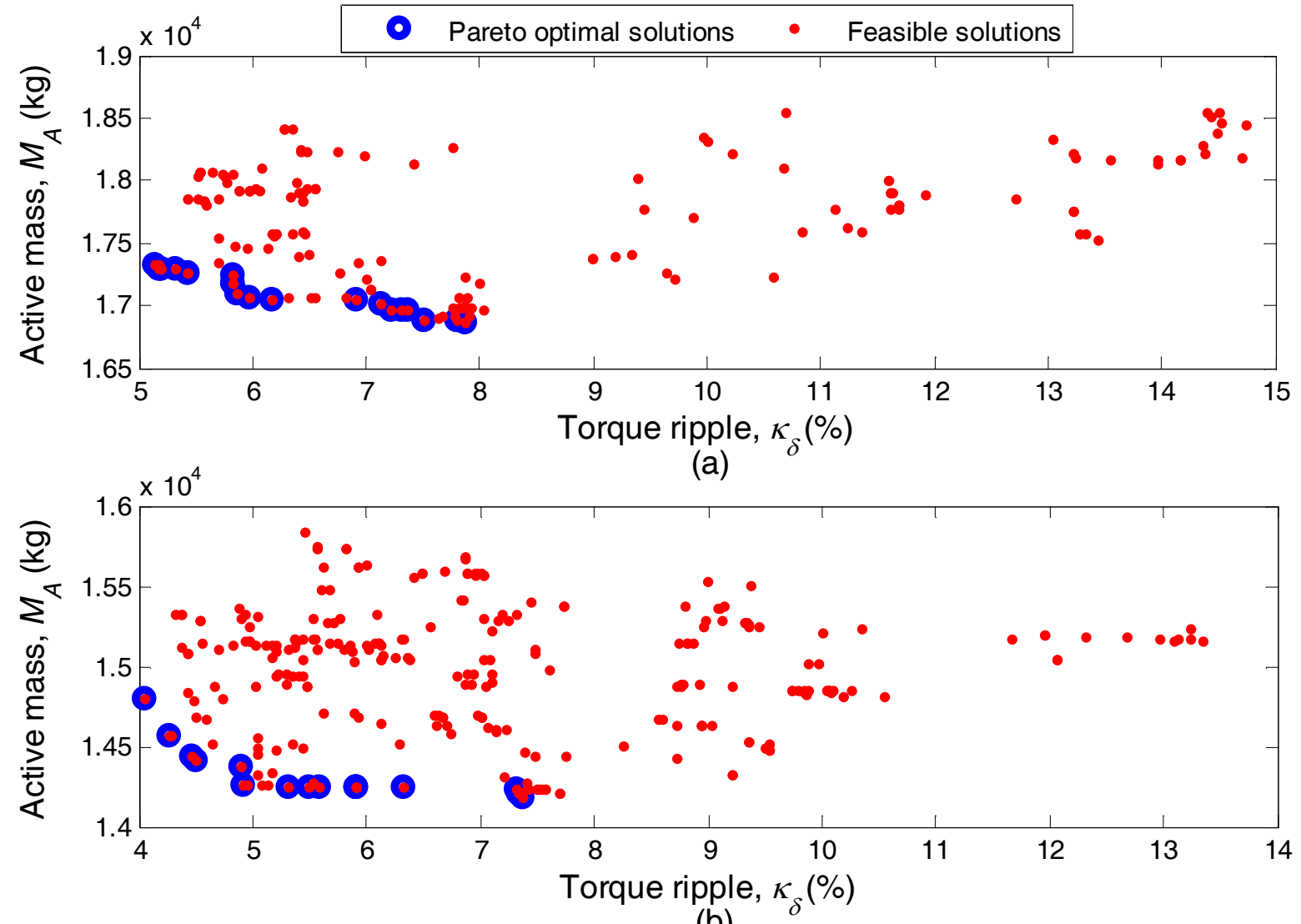

(b)

Fig. 6.4. Pareto optimal fronts for different 3 MW ferrite PM-FSMs: (a) 12/10, and (b) 12/14. 

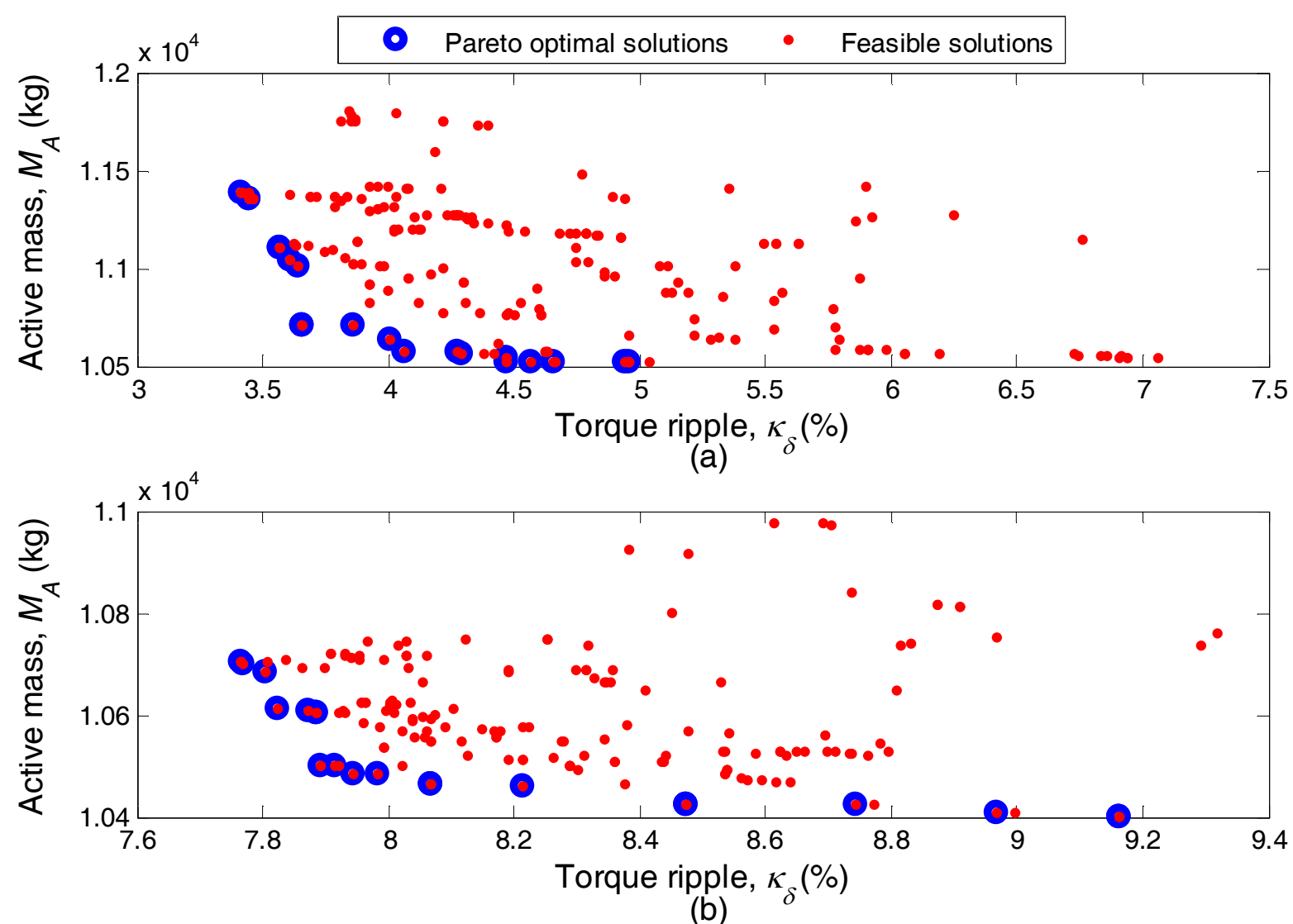

(b)

Fig. 6.5. Pareto optimal fronts for different 3 MW WF-FSMs: (a) 12/10, and (b) 12/14.

Furthermore, it is observed that a better mass minimisation occurs in the WF-FSMs compared to the ferrite PM-FSMs at $3 \mathrm{MW}$ power levels by at least $34 \%$ and $28 \%$, for the $12 / 10$ and $12 / 14$ machines, respectively. This is a very crucial find, especially given that the copper windings in the WF-FSMs were modelled to very realistic working temperatures $\left(80^{\circ} \mathrm{C}\right)$, unlike for the ferrite PMFSMs which were kept at room temperature conditions.

To discover the reason for the significant active mass minimisation of the WF-FSM compared to ferrite PM-FSMs at 3 MW power levels, eight designs are initially benchmarked from each of the obtained optimal solution sets and compared as shown in Tables 6.2 and 6.3. Based on the values reported, one can observe that the discrepancy in mass, between the optimal ferrite PM-FSM and WFFSM designs at $3 \mathrm{MW}$ power levels, is influenced by interactions between the armature current density and aspect ratio. In response to the set design optimisation targets, it is noticed that the aspect ratios of the ferrite PM-FSMs at 3 MW power increases drastically, whereas their current densities decreases in tandem, compared to their $10 \mathrm{~kW}$ machines. However, the same cannot be said for the 3 MW WF-FSMs, whereby it is observed that fairly similar values are maintained when both parameters are compared accordingly. Thus, based on the set of complete design points operated during the MDO process, both feasible and infeasible, a linear relationship between the armature current density 
and aspect ratio has been correlated for the $3 \mathrm{MW}$ rare-earth-free machines as shown in Fig. 6.6, which confirms their optimal behaviors, given the freedom of the MDO design space. Note that, the active optimal solutions of the ferrite PM-FSM and WF-FSM at $3 \mathrm{MW}$ power levels are mainly situated in the region of current density values of $1-1.5 \mathrm{~A} / \mathrm{mm}^{2}$ as shown in Fig. 6.6.

Generally, Table 6.2 also reveals that the 12/14 machines result in higher torque per active stack volumes compared to the 12/10 machines, irrespective of the type of rare-earth-free excitation or operating power level. The 3 MW rare-earth PM-FSMs imported from the study earlier undertaken in Chapter 3, presents an exception to the case, whereby a very sharp difference indicated for their armature current densities is the main culprit.

In terms of total cost of active materials, the 12/14 rare-earth-free machines are thus cheaper compared to their 12/10 machines as indicated. Again, the exception to the case, i.e., the $10 \mathrm{~kW}$ 12/14 WF-FSM benchmark, is likely due to the output power exceeding the optimal $10 \mathrm{~kW}$ design threshold. On the other hand, comparing the ferrite PM-FSM and WF-FSM shows that the cost of the latter is higher for the $10 \mathrm{~kW}$ machines and lower for the $3 \mathrm{MW}$ machines due to the tradeoffs observed in their total active mass at the different power levels.

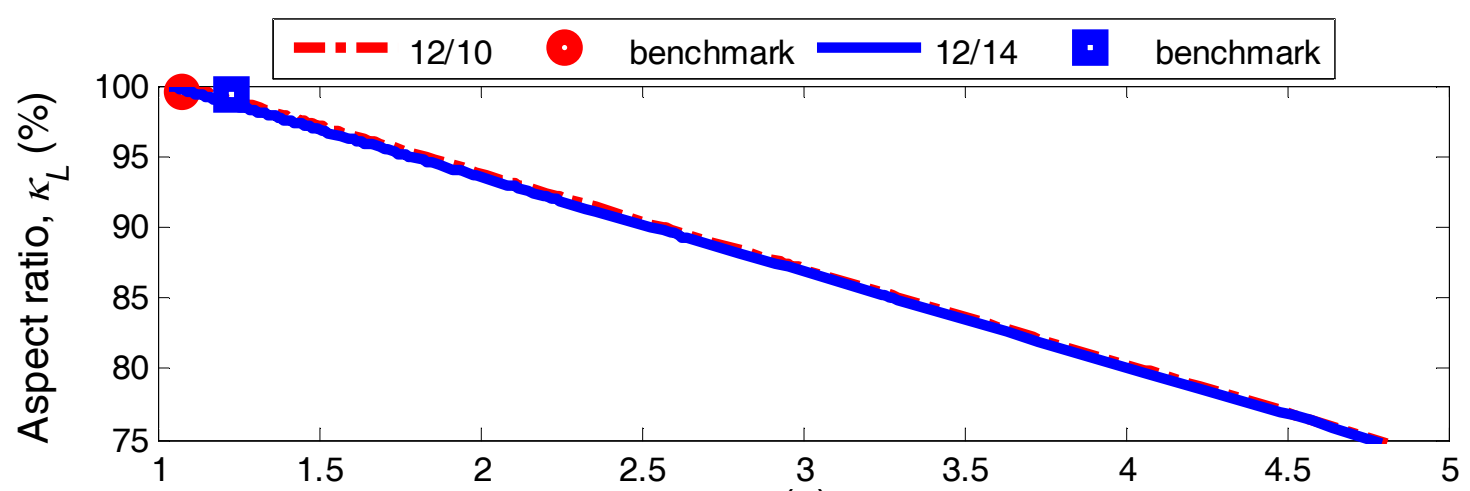

(a)

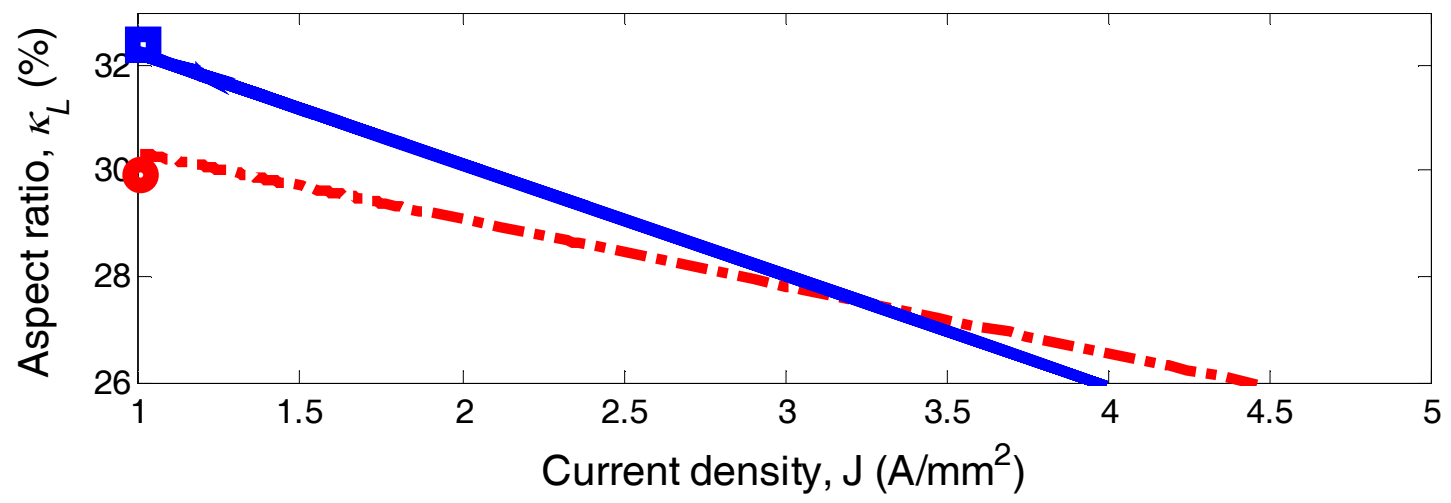

(b)

Fig. 6.6. Optimal partnership between current density and aspect ratio in $3 \mathrm{MW}$ rare-earth-free FSMs: (a) ferrite PM-FSM, and (b) WF-FSM. 
Table 6.2. Performance comparison of different machine characteristics for geared medium-speed wind generators

\begin{tabular}{|c|c|c|c|c|c|c|c|c|c|c|c|c|c|}
\hline & Design & $\begin{array}{l}\mathrm{P}_{\text {out }} \\
\mathbf{k W}\end{array}$ & $\begin{array}{c}\tau_{e} \\
\mathbf{N m}\end{array}$ & $\begin{array}{l}\kappa_{\delta} \\
\%\end{array}$ & $\begin{array}{c}M_{F e S} \\
\mathbf{k g}\end{array}$ & $\begin{array}{c}M_{F e R} \\
\mathbf{k g}\end{array}$ & $\begin{array}{c}M_{P M / F} \\
\mathrm{~kg}\end{array}$ & $\begin{array}{c}M_{C u} \\
\mathbf{k g}\end{array}$ & $\begin{array}{c}M_{A} \\
\mathbf{k g}\end{array}$ & $\begin{array}{l}\eta \\
\%\end{array}$ & $\begin{array}{c}\mathrm{PF} \\
-\end{array}$ & $\begin{array}{c}\tau_{e} / V_{A} \\
\mathrm{kNm} / \mathbf{m}^{3}\end{array}$ & $\begin{array}{c}\operatorname{Cost}^{24} \\
\text { SUS }\end{array}$ \\
\hline \multirow{4}{*}{ 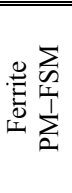 } & $12 / 10$ & 10.017 & 259.563 & 3.384 & 34.164 & 20.540 & 11.201 & 7.576 & 73.481 & 94.927 & 0.805 & 17.311 & 317.210 \\
\hline & $12 / 14$ & 10.067 & 260.988 & 2.432 & 28.096 & 22.474 & 9.114 & 7.266 & 66.951 & 96.186 & 0.811 & 18.701 & 283.773 \\
\hline & $12 / 10$ & 3063.398 & 79246.694 & 5.153 & 9155.799 & 4023.046 & 2531.542 & 1617.292 & 17327.681 & 99.111 & 0.805 & 19.953 & 72422.549 \\
\hline & $12 / 14$ & 3011.434 & 78044.441 & 4.363 & 7253.789 & 4378.919 & 2040.463 & 1124.986 & 14798.159 & 99.018 & 0.810 & 22.197 & 58596.431 \\
\hline \multirow{4}{*}{$\sum_{\substack{\infty \\
1 \\
1 \\
3}}$} & $12 / 10$ & 9.995 & 249.469 & 5.877 & 67.352 & 42.648 & 8.854 & 11.999 & 130.856 & 89.090 & 0.826 & 8.639 & 475.553 \\
\hline & $12 / 14$ & 11.371 & 295.537 & 9.957 & 62.005 & 42.978 & 9.851 & 14.086 & 128.921 & 89.296 & 0.790 & 10.309 & 499.057 \\
\hline & $12 / 10$ & 2998.164 & 77841.826 & 3.736 & 6575.027 & 2913.221 & 685.939 & 1040.134 & 11214.322 & 97.017 & 0.804 & 31.586 & 40206.163 \\
\hline & $12 / 14$ & 3075.991 & 79616.178 & 8.733 & 5660.582 & 3474.238 & 587.632 & 877.086 & 10599.540 & 97.429 & 0.799 & 34.354 & 36501.445 \\
\hline \multirow{2}{*}{$\stackrel{\sim}{\gtrless}$} & $12 / 10$ & 3018.262 & 78354.538 & 1.344 & 2363.538 & 1751.821 & 619.729 & 314.650 & 5049.740 & 98.875 & 0.823 & 58.917 & 49761.609 \\
\hline & $12 / 14$ & 3044.434 & 78908.753 & 1.283 & 3081.062 & 1573.498 & 697.132 & 535.802 & 5887.495 & 99.103 & 0.832 & 57.088 & 58068.930 \\
\hline \multirow[b]{2}{*}{ జึ } & PMSG & 3000.000 & 318309.886 & - & \multirow{2}{*}{\multicolumn{2}{|c|}{$\begin{array}{l}4370.000 \\
8650.000\end{array}$}} & 410.000 & 1330.000 & 6110.000 & 98.000 & - & 78.179 & 49110.000 \\
\hline & DFIG & 3000.000 & 318309.886 & - & & & \multicolumn{2}{|c|}{2720.000} & 11370.000 & 97.000 & - & 52.119 & 49494.000 \\
\hline
\end{tabular}

Table 6.3. Comparison of some basic optimal design parameters

\begin{tabular}{|c|c|c|c|c|c|c|c|c|c|c|}
\hline & Design & $\begin{array}{c}l_{s t} \\
\mathbf{m m}\end{array}$ & $\begin{array}{c}D_{\text {in }} \\
\mathbf{m m}\end{array}$ & $\begin{array}{l}D_{\text {out }} \\
\text { mm }\end{array}$ & $\begin{array}{l}\Lambda_{0} \\
\% \\
\end{array}$ & $\begin{array}{l}\boldsymbol{\kappa}_{L} \\
\% \\
\end{array}$ & $\begin{array}{c}\mathbf{J} \\
\mathbf{A} / \mathbf{m m}^{2} \\
\end{array}$ & $\begin{array}{c}\mathbf{J}_{\mathbf{F}} \\
\mathbf{A} / \mathbf{m m}^{2} \\
\end{array}$ & $\begin{array}{c}\boldsymbol{g} \\
\mathbf{m m}\end{array}$ & $\begin{array}{c}n_{s} \\
\mathbf{r} / \mathbf{m i n} \\
\end{array}$ \\
\hline \multirow{4}{*}{ 导 } & $12 / 10$ & 139.415 & 234.875 & 370.043 & 63.472 & 59.357 & 4.607 & - & 0.700 & 360 \\
\hline & $12 / 14$ & 144.888 & 235.033 & 350.201 & 67.113 & 61.645 & 3.824 & - & 0.700 & 360 \\
\hline & $12 / 10$ & 1311.451 & 1318.522 & 1963.627 & 67.147 & 99.463 & 1.079 & - & 2.756 & 360 \\
\hline & $12 / 14$ & 1316.814 & 1325.971 & 1843.780 & 71.915 & 99.309 & 1.228 & - & 2.624 & 360 \\
\hline \multirow{4}{*}{$\sum_{\substack{\infty \\
1 \\
1}}^{1}$} & $12 / 10$ & 97.135 & 435.244 & 615.212 & 70.746 & 22.317 & 1.434 & 4.958 & 0.700 & 360 \\
\hline & $12 / 14$ & 97.650 & 429.558 & 611.380 & 70.260 & 22.732 & 1.224 & 4.918 & 0.700 & 360 \\
\hline & $12 / 10$ & 507.185 & 1695.249 & 2487.295 & 68.156 & 29.918 & 1.004 & 4.999 & 3.000 & 360 \\
\hline & $12 / 14$ & 540.303 & 1668.408 & 2336.922 & 71.393 & 32.384 & 1.014 & 4.994 & 3.000 & 360 \\
\hline \multirow{2}{*}{$\varangle$} & $12 / 10$ & 702.434 & 1234.381 & 1552.608 & 79.503 & 56.905 & 4.975 & - & 2.514 & 360 \\
\hline & $12 / 14$ & 693.796 & 1152.776 & 1592.681 & 72.379 & 60.184 & 2.087 & - & 2.995 & 360 \\
\hline \multirow{2}{*}{$\infty$} & PMSG & 400.000 & - & 3600.000 & - & - & - & - & 3.600 & 90 \\
\hline & DFIG & 600.000 & - & 3600.000 & - & - & - & - & 2.000 & 90 \\
\hline
\end{tabular}

24 The cost estimations are performed based on quotations in US dollars (USD) mined from Fasolo, Alberti and Bianchi (2014) [69].

25 Rare-earth PM-FSM benchmarks imported from Table 3.4 in Chapter 3.

26 A selection of some conventional wind generator topologies from literature. 
The latter rows in Tables 6.2 and 6.3 are provided to highlight the comparative performance characteristics of the rare-earth PM-FSMs earlier considered in Chapter 3 and other traditional geared MS wind generators sourced from the literature, viz., PMSG and DFIG, in respect of the rareearth-free FSM design candidates at 3 MW power levels only. Information on the key data for the PMSG and DFIG machines were retrieved from the study by Polinder et al (2006) [11]. With this additional information, it is clearly shown that the cheapest generator active material cost at $3 \mathrm{MW}$ power is expressed by the WF-FSM, compared to the rest. Notwithstanding, the PMSG, rare-earth PM-FSM and DFIG systems produce significant torque densities compared to the WF-FSM. This is because the PMSG and DFIG are designed at lower rated speed and perhaps with large split and small aspect ratios as indicated in their stator outer diameters and stack lengths shown in Table 6.3. Whereas, it should also be noted that the rare-earth PM-FSM, in addition to the PMSG, are both designed using high-energy quality rare-earth PMs. Yet, it has to be reiterated that the main interest in the current study is to despise the use of more expensive rare-earth PM designs such as the PMSG and the rare-earth PM-FSM, while on the other hand, for certain DFIGs, the generator maintenance costs are exacerbated due to their usage of slip rings and brushes.

Meanwhile, observe that the concentration of the optimum design solutions of the ferrite PMFSM on lower armature current densities and higher split ratios resulted in them being the most expensive designs at $3 \mathrm{MW}$ power levels. Based on the NSGA-II optimisation algorithm used in this study, the unlikely situation obtained for the ferrite PM-FSM at utility-scale power levels could be resolved if only the margin of the aspect ratio is set to smaller values (75-85\%) while alternatively increasing the margin of the split ratio before the MDO process is initiated.

Lastly, it is equally observed from Table 6.2 that the $3 \mathrm{MW}$ machine credited with the highest cost of generator active material and lowest torque density, viz., the ferrite PM-FSM, yields the best generator efficiency at over $99 \%$, with the rare-earth PM-FSM following closely. But considering the fact that the copper resistivity used in the design process of the ferrite and rare-earth PM-FSMs were approximated at much lower temperature coefficients than for the WF-FSM, the purported achievement of high efficiency is therefore not conclusive. But, it should be noted that the presence of the wound-fields in the WF-FSMs is an additional burden on the efficiency requirements.

Thus, the preferred solution for the proposed rare-earth-free wind turbine generator, especially at utility-scale, might as well be nominated from the $3 \mathrm{MW}$ WF-FSM design candidates. To this end, both $3 \mathrm{MW}$ WF-FSM design candidates are used to substantiate the 2-D static FEA predictions, so far made, in a corresponding 3-D transient FEA evaluation in the ensuing section. 


\subsection{Comparison of 2-D and 3-D FEA Results}

As formerly proclaimed, the overlapping of the phase coils over the wound-field coils may portray significant effects of the end-windings in the WF-FSMs compared to the ferrite PM-FSMs studied here. Moreover, considering the fact that the current study has been used to report the performance of these machines at utility-scale power levels, it is therefore critical to establish the accuracy of the results obtained in 2-D static FEA by performing additional 3-D transient FEA modelling and simulations based on the ANSYS Maxwell ${ }^{\odot}$ software. Thus, the different WF-FSMs benchmarked at 3 MW power in the preceding section are nominated for the procedure.

The magnetic field distributions of the models earlier analysed in 2-D static FEA, as well as their magnetic flux density map in 3-D transient FEA, are displayed as shown in Fig. 6.7, each individually evaluated at rated condition. Without doubt, the end-leakages are highly dominant in the 3D models as seen on all its sides, including the surfaces defined at both ends of the axial path, as well as in the rotor shaft region, compared to the 2-D models. As a result, a decrease in the torque capability in the 3-D models as later compared in Table 6.4 is accepted at face value.

The on-load phase flux linkages evaluated in 2-D and 3-D FEA are compared as shown in Fig. 6.8. The 12/14 machine displayed good confidence with $2.2 \%$ discrepancy between 2-D and 3-D FEA solutions. However, a the higher discrepancy (17.5\%) is observed in the 12/10 machine due to the fact that the clearances of the phase end-winding from the end sections of the lamination stack, imposed at the design stage in $2-\mathrm{D}$, were slightly underestimated.

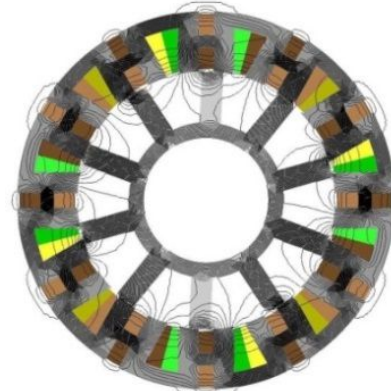

(a)

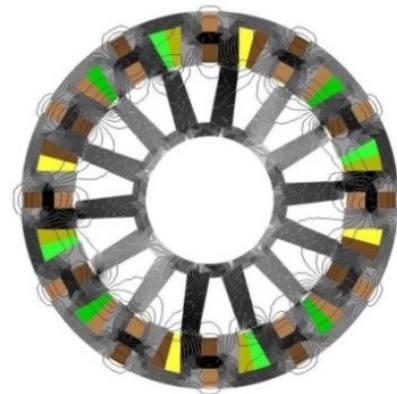

(c)

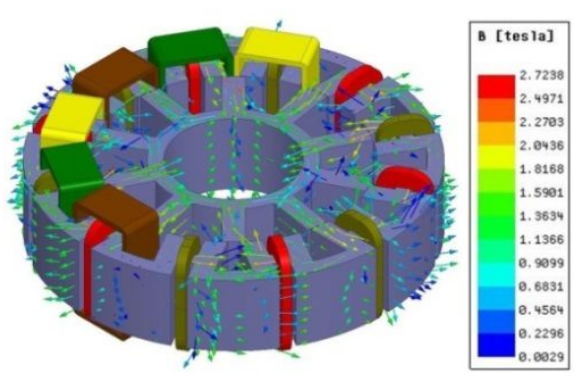

(b)

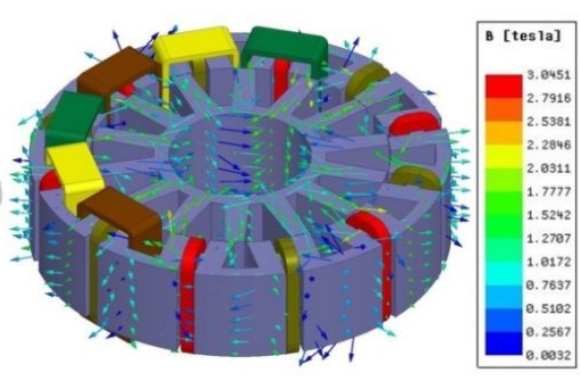

(d)

Fig. 6.7. Magnetic flux distributions and densities of the compared $3 \mathrm{MW}$ WF-FSMs displayed in: (a) 12/10 2-D model, (b) 12/10 3-D model, (c) 12/14 2-D model, and (d) 12/14 3-D model. 


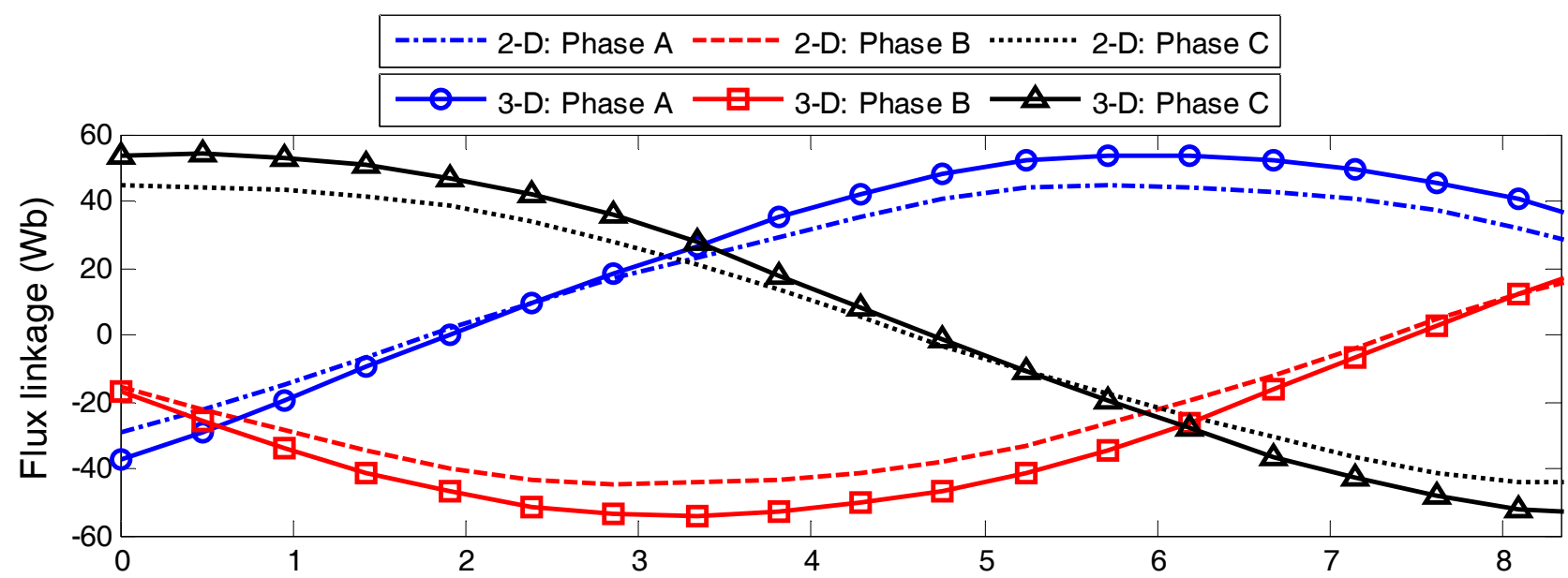

(a)

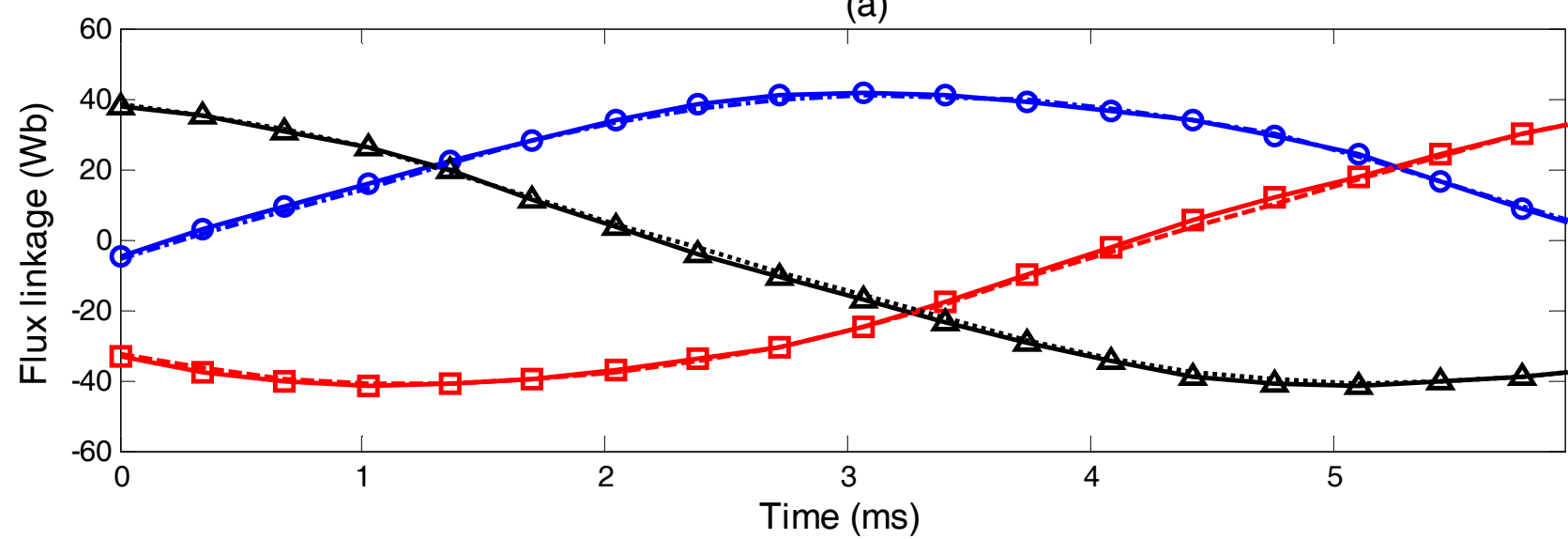

(b)

Fig. 6.8. On-load phase flux linkage waveforms plotted over half electrical period in 3 MW WFFSMs: (a) 12/10 design, and (b) 12/14 design.

Table 6.4. Comparison of performance characteristics of $3 \mathrm{MW}$ WF-FSMs in 2-D and 3-D FEA

\begin{tabular}{c|c|c|c|c|c|c}
\hline \multirow{2}{*}{ Design } & \multicolumn{2}{|c|}{$\boldsymbol{\tau}_{\boldsymbol{e}}(\mathbf{k N m})$} & \multicolumn{2}{c|}{$\boldsymbol{P}_{\boldsymbol{C u}}(\mathbf{k W})$} & \multicolumn{2}{c}{$\boldsymbol{\kappa}_{\boldsymbol{\delta}}(\mathbf{\%})$} \\
\cline { 2 - 7 } & 2-D FEA & 3-D FEA & 2-D FEA & 3-D FEA & 2-D FEA & 3-D FEA \\
\hline \hline $12 / 10$ & 77.841 & 72.949 & 68.513 & 67.299 & 3.736 & 23.166 \\
\hline $12 / 14$ & 79.616 & 71.617 & 52.951 & 52.561 & 8.733 & 16.923 \\
\hline
\end{tabular}

Table 6.4 is also used to give account on the average electromagnetic torque, torque ripple and total copper loss readings evaluated in both 2-D and 3-D FEA. As earlier insinuated, the differences observed in the torque values is due to higher end leakage effects observed in the 3-D designs compared to 2-D, whereas the different mesh densities applied in 2-D (finer) and 3-D (coarser) may be primarily implicated for the observed discrepancy in torque ripple values.

\subsection{Chapter Summary}

In summary, the researcher has been able to present in this chapter, the multi-objective optimal design characteristics of rare-earth-free FSMs based on 2-D static FEA studies. The rare-earth-free 
schemes reported are the ferrite PM-FSM and WF-FSM, which were both investigated in their 12/10 and 12/14 configurations. To the researcher's best knowledge, this is the very first time these machine configurations have been designed and subjected to this kind of study. The machines have been proposed for geared MS wind generator drives and in order to escalate the potentials for utility-scale applications, the study was implemented in small-scale $(10 \mathrm{~kW})$ as well as in large-scale (3 MW) power levels. From the design optimisation results, the following general findings are made:

- The 12/14 ferrite PM-FSMs yield lower active mass and torque ripples compared to the $12 / 10$ machines, at both $10 \mathrm{~kW}$ and $3 \mathrm{MW}$ power levels, although the decrease is more significant in terms of the active mass at $3 \mathrm{MW}$ power levels.

- For the WF-FSM, the outcome is different at both power levels considered, whereby it is observed that the $12 / 10$ machines can achieve significant lower torque ripple values than the $12 / 14$ machines, with slightly smaller active mass evinced in the former.

- The presence of the wound-field coils as well as the vulnerability of the 12/14 machines to higher core loss, is found to further limit the efficiency requirements of the WF-FSM variants.

- At $10 \mathrm{~kW}$ power, the ferrite PM-FSM performs better in terms of lowest torque ripple and active mass for both machine configurations. But at $3 \mathrm{MW}$ power, the WF-FSMs have better torque densities, while the ferrite PM-FSMs have better torque ripple values.

- In addition, considering the results obtained in Chapter 3 for rare-earth PM-FSMs, there seem to be a general misconception that rare-earth-free designs would perform better in terms of their torque ripple, but which obviously is not the case (see Figs. 3.2 and 3.3 in Chapter 3 and compare with Figs. 6.2-6.5). Apparently there appears to be little support for implicating the flux focusing abilities of FSMs for their characteristically high torque ripple effects as mentioned, for example, in Fei, Luk and Shen (2012) [144] and Zhu et al (2017) [145].

- Overall, the minimisation of the total active mass is better propagated in the $3 \mathrm{MW} \mathrm{12/10}$ and 12/14 WF-FSM configurations compared to the ferrite PM-FSMs at the same power level.

Based on these findings, eight optimal designs were benchmarked, whereby it was further found that the 12/14 machines generally exhibited higher torque per active stack volumes and are cheaper in terms of active material costs. However, between the ferrite PM-FSM and WF-FSM, the active material cost of the latter is higher for small-scale $(10 \mathrm{~kW})$ machines but significantly lower for large-scale (3 MW) machines, which is due appropriate balancing of the optimal aspect ratio and armature current density in response to the optimum design requirements. Essentially, it was found 
that there is an inherent tradeoff between the aspect ratio and armature current density of such rareearth-free FSMs which influences their total active mass, especially at the utility-scale power levels. Consequently, to ensure the feasibility of the optimal design, there is need to appropriately control the margins of the aspect and split ratios before engaging them in a MDO procedure.

Furthermore, by comparing the rare-earth-free optimum design candidates in the $3 \mathrm{MW}$ power range with their counterpart rare-earth PM-FSMs, as well as some other traditional geared MS wind generators, viz., DFIG and PMSG, it was found that the generator with the cheapest active material cost remains that of the WF-FSM, although the DFIG and PMSG systems were still capable of higher torque densities. Despite having the lowest torque density, the ferrite PM-FSM, recorded the best efficiency at over $99 \%$ compared to the rest, thanks to sacrificing higher armature current densities for longer stacked machines in response to the design requirements. Followed closely, is the rareearth PM-FSMs, which also acquired efficiencies at $99 \%$ due to better tradeoffs in terms of its optimal aspect and split ratios, among other things. But these outcomes are not to be relied upon since the theoretical temperature operated for the PM-FSM designs are far less than that designated to the WF-FSMs, which apparently are armed with both field and armature coils.

Eventually, the WF-FSM is nominated as the preferred solution for the proposed rare-earthfree wind turbine generator drivetrain, especially for utility-scale applications. Based on this, the two selections which were earlier benchmarked from the 3 MW WF-FSM optimal design candidates were promoted for further 3-D transient FEA evaluations.

In 3-D FEA, the $3 \mathrm{MW} \mathrm{12/14} \mathrm{WF-FSM} \mathrm{design} \mathrm{candidate} \mathrm{displayed} \mathrm{very} \mathrm{good} \mathrm{confidence} \mathrm{(2.2}$ $\%$ ) in line with its 2-D FEA predictions based on the on-load phase flux linkages, whereas a $17.5 \%$ discrepancy is observed in the 12/10 machine under the similar operating conditions. The larger disagreement in the latter is due to apparent underestimation of the end-winding effect at the 2-D FEA design stage. Lastly, compared to 2-D, the torque capabilities of both 3-D models were slightly decreased, due to the eminence of end leakage effects as observed in their corresponding flux density maps. 


\section{Chapter Seven}

\section{Prototype Development and Testing}

In this chapter, the development of a $10 \mathrm{~kW}$ WF-FSM designed for the proposed wind generator drivetrain is articulated. The prototype is a byproduct of the MDO process escalated in Chapter 5. The highlight of this chapter is the promotion of ease in the manufacturing process such that modular structures of the stator laminations, preformed coils of the field (DC) and phase (AC) windings, as well as coupling of the rotor stack unto a rotor hub, are implemented. Beyond all these, it is the first time an experimental test is being conducted on one of the variants of the proposed WF-FSM configurations - the 12/10 machine - to the best of the researcher's knowledge. Based on the final test results, the confidence level of the FEA predictions made in this dissertation is established.

\subsection{Introduction}

Various FSM topologies (the 12/10 versus 12/14 machines; rare-earth PM versus rare-earth-free topologies; small-scale versus large-scale power levels, etc.) have been analysed and proposed for geared MS wind generator drives in this dissertation, by using FEA design and non-gradient optimisation techniques. The core interest has been on the electromagnetic design of the wind generator among other drivetrain components like the gearbox and SSCs, among others. Consequently, one of the optimal design candidates, as processed in the study conducted in Chapter 5, has been nominated for prototyping and experimental tests - the so-called 12/10 $10 \mathrm{~kW}$ WF-FSM.

In literature accounts, the said candidate design, i.e., the 12/10 WF-FSM was first highlighted by the researcher in full-scale design, optimisation and analyses as disclosed in the previous chapters. The only other study where the reference topology was mentioned, but in passing, is in Tang et al (2012) [67]. Although credit must be given to Tang et al (2012) [67] for first proposing these WFFSM topologies, however their study only proceeded on that of the 12/14 machine strictly based on FEA studies and no design and optimisation performed. Ever since then, no other researcher, in the researcher's best opinion, has considered these WF-FSM topologies. Perhaps, the reason why further development on these WF-FSM topologies has been suppressed is due to a premeditated fear regarding its manufacturing woes, which again, to the researcher's understanding, can be substantiated. In terms of its performance, Tang et al's (2012) [67] study clearly showed the potentials of these WFFSMs to yield comparable torque qualities and maximum field weakening abilities compared to their PM-FSM counterparts. Thus, the procedure documented in this chapter, assumedly second to none, 
is actually a proof of the concept.

Furthermore, the choice of the WF-FSM above the ferrite PM-FSM, has been influenced by the fact that it not only presents a brushless wind generator topology when compared to conventional wound-field machines such as the WRSGs and DFIGs, but it entirely eliminates the fear of demagnetisation in high temperature operating conditions. Thus, its stator-mounted nature implies that the heat produced by its windings can be easily managed. Also, such topologies offer field control strategies unlike their PM topologies, with increased benefits to variable-speed wind generator applications whereby the intermittent wind resource can be regulated in tandem with the field supply to improves the overall load efficiency of the wind turbine over a wide power range, Marques et al: 2003 [136] and Muller, Deicke and de Doncker: 2002 [137].

A block diagram of the proposed layout of the experimental test-bench is displayed as shown in Fig. 7.1. Note that, although the gearbox is not represented in this schematic, the geared MS speed terrain is notwithstanding assumed by the operation of the experimental prime mover.

\subsection{Prototype Presentation and Artistic Impression}

As earlier mentioned, the prototype presented in this chapter was conceived from the design optimisation process in Chapter 5. The main design parameters of the $10 \mathrm{~kW}$ WF-FSM benchmark is given in Table 7.1, while the materials adopted are specified in Table 7.2.

To facilitate the manufacturing of the $10 \mathrm{~kW}$ WF-FSM prototype which is considered unprecedented by the researcher, an artistic impression of the complete drivetrain structure is created as shown in Fig. 7.2. Fig. 7.3, furthermore, shows the exploded 3-D views of both the stator and the rotor assembly. Consequently, to construct the different components required for the test-bench generator, a three-stage manufacturing process such as laser cutting, machining and welding, was initiated.

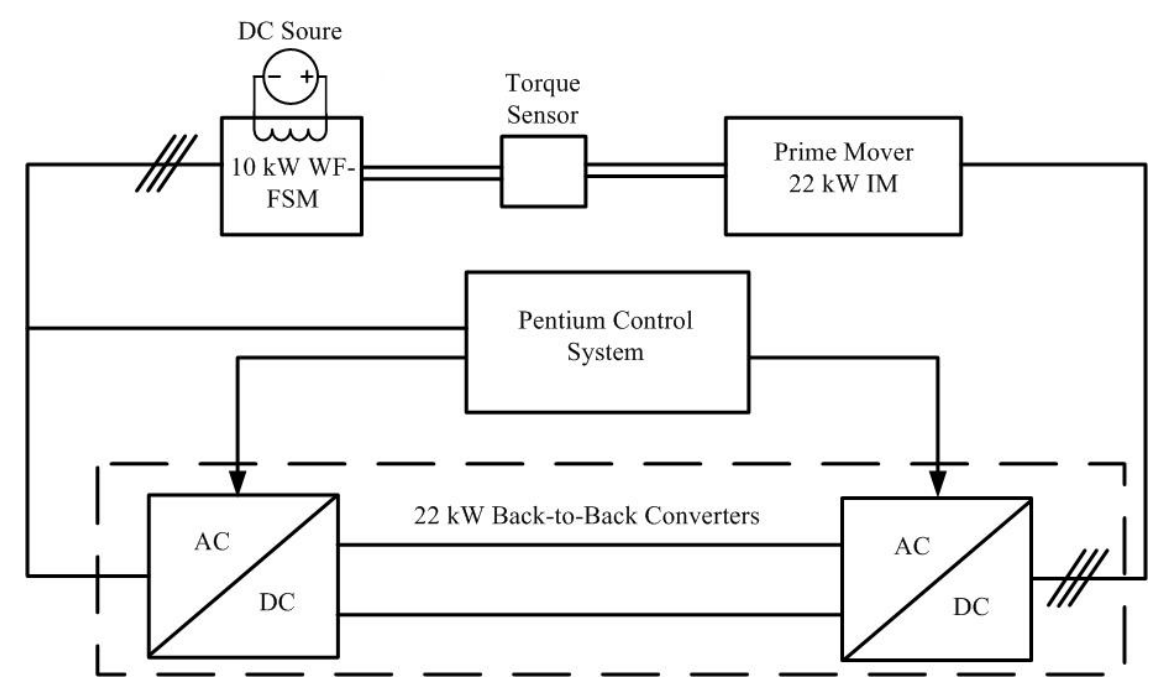

Fig. 7.1. A block diagram of the drivetrain test-bench for the $\overline{10 \mathrm{~kW} \mathrm{WF}} \overline{-F S M}$ prototype. 
Table 7.1. Main design parameters of $10 \mathrm{~kW}$ WF-FSM prototype

\begin{tabular}{c|c|c}
\hline & Parameters & WF-FSM \\
\hline \hline$\alpha$ & Current angle (deg.) & 89.962 \\
$\mathrm{~J}_{F}$ & Field current density $\left(\mathrm{A} / \mathrm{mm}^{2}\right)$ & 4.963 \\
$D_{\text {out }}$ & Stator outer diameter $(\mathrm{mm})$ & 600 \\
$l_{s t}$ & Stack length $(\mathrm{mm})$ & 104.069 \\
$D_{i n}$ & Stator inner diameter $(\mathrm{mm})$ & 415.412 \\
$D_{s h}$ & Shaft diameter $(\mathrm{mm})$ & 227.073 \\
$h_{c F}$ & Field core iron height $(\mathrm{mm})$ & 29.474 \\
$b_{F}$ & Field core iron width $(\mathrm{mm})$ & 33.717 \\
$b_{p r}$ & Rotor pole width $(\mathrm{mm})$ & 64.153 \\
$b_{s l s}$ & Slot opening width $(\mathrm{mm})$ & 24.336 \\
$h_{y s}$ & Stator yoke height $(\mathrm{mm})$ & 32.924 \\
$h_{y r}$ & Rotor yoke height $(\mathrm{mm})$ & 39.999 \\
$t_{0}$ & Rotor teeth taper factor & 0.754 \\
$g$ & Airgap thickness $(\mathrm{mm})$ & 0.7 \\
$\mathrm{~J}$ & Phase current density $\left(\mathrm{A} / \mathrm{mm}^{2}\right)$ & 1.644 \\
\hline
\end{tabular}

Table 7.2. Materials used in the design of $10 \mathrm{~kW}$ WF-FSM prototype

\begin{tabular}{c|c}
\hline Material & Properties \\
\hline Windings & Copper: $\rho_{C u}=2.07312 \times 10^{-8} \Omega \mathrm{m}$ \\
Laminations & NO fully processed electrical steel: M400-50A \\
\hline
\end{tabular}

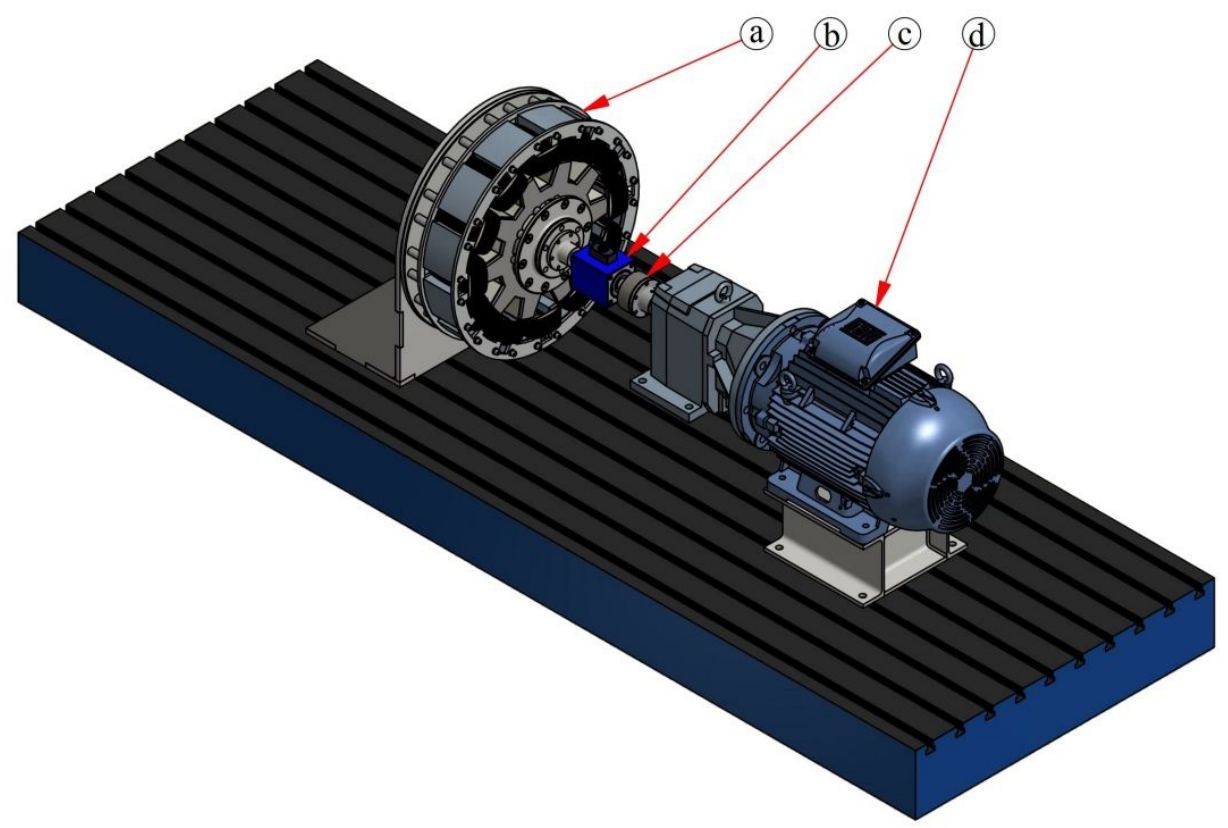

Fig. 7.2. An artistic impression of the proposed test-rig with highlights on: (a) the $10 \mathrm{~kW}$ WF-FSM, (b) the torque sensor, (c) the coupling device, and (d) the $22 \mathrm{~kW}$ IM prime--mover. 


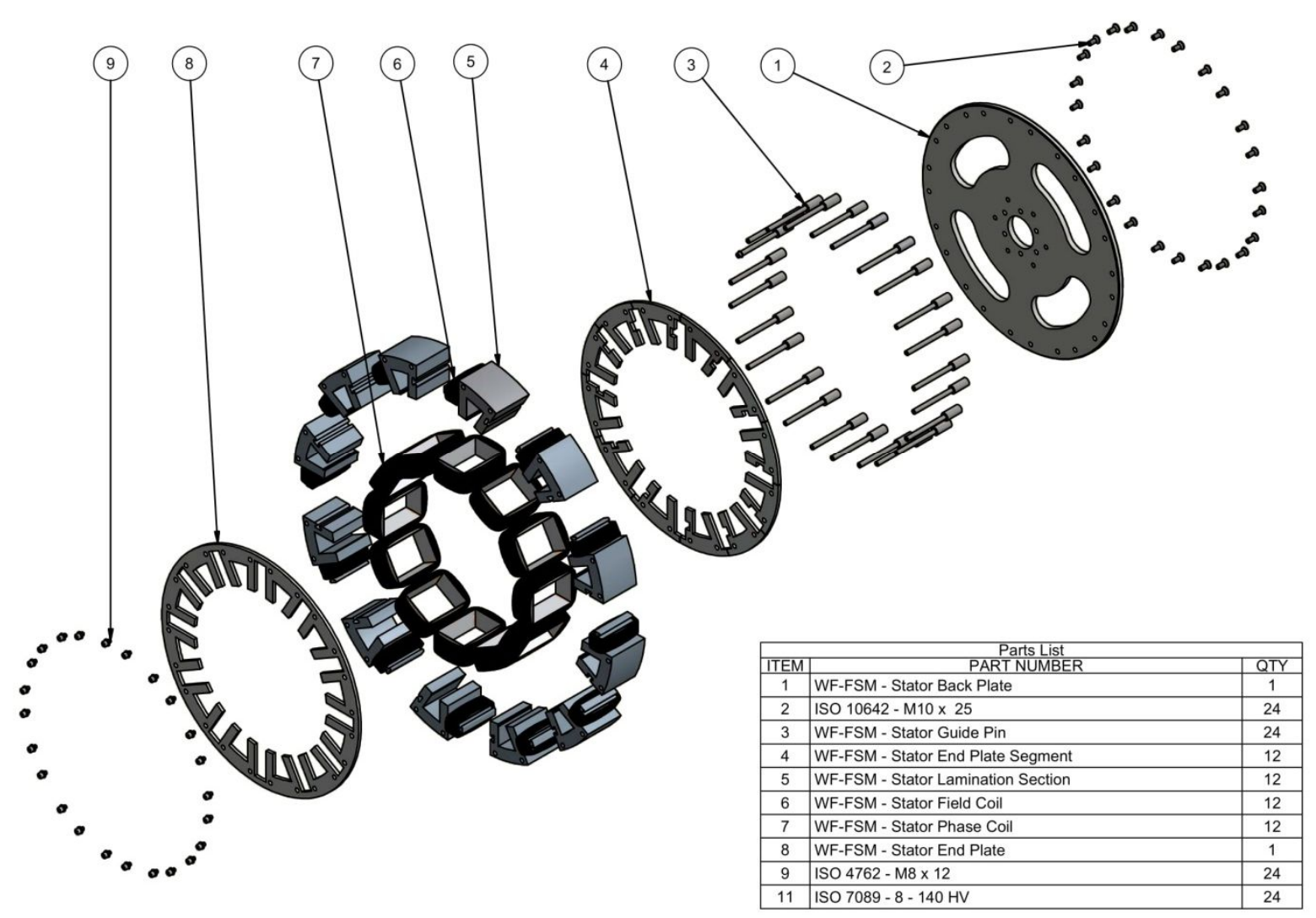

(a)
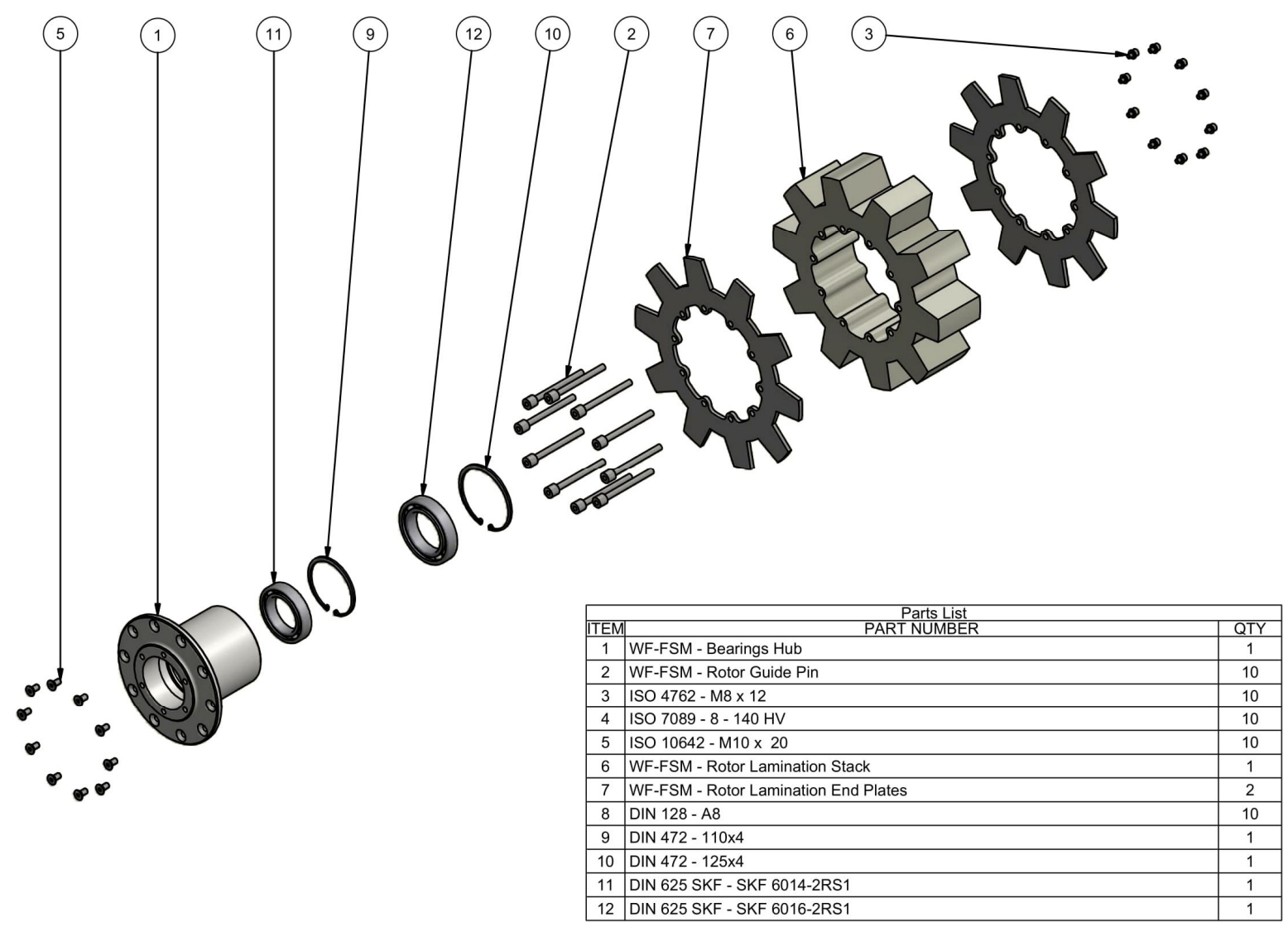

(b)

Fig. 7.3. 3-D exploded views showing the different components in: (a) the stator assembly, and (b) the rotor assembly. 
Some of the materials manufactured by laser cutting include the rotor and stator laminations. In order to alleviate a potential difficulty in assembling of the preformed wound-fields, the modular structure of the stator laminations in typical PM-FSMs is adopted for the WF-FSM prototype. Also, a unique contraption in a puzzle-like assembly is introduced, whereby 12 pieces of the iron laminations are assembled circumferentially to produce a single-layer of the lamination ring. In this manner, the design is also able to achieve structural stability. Some samples and processes of the manufactured components as developed for the $10 \mathrm{~kW}$ WF-FSM prototype are shown in Figs. 7.4-7.6. Note that, the rotor lamination remains, as is always the case in FSMs, a robust piece of iron. Also note that, the holes punched at the perimeter edges of both the outer stator and inner rotor lamination modules are done in order to reinforce their assembly stacking, with negligible effect on the electromagnetic performance (about $1 \%$ ).

Owing to the current and voltage limitation of the DC power supply and back-to-back converters available for the experimental tests, the researcher resorted to certain parallel connections for both the wound-fields (DC coils) and the three-phase windings (AC coils). The devised winding layouts are illustrated in Figs. 7.7 and 7.8, with the summary data provided in Table 7.3.

\subsection{Test Results and Discussions}

Upon completion of the machine assembly, the main experimental test-bench is set up for testing the prototype wind generator as shown in Fig. 7.9. The torque sensor is meant to measure the mechanical input torque and speed of the generator when operated, while the commonly used $I_{d}=0$ control strategy is implemented via the Pentium system. Consequently, basic no-load, short-circuit, temperature and uncontrolled resistance tests, as well as simple implementation of the field-oriented control are further escalated in this section.

\subsubsection{No-Load tests}

To begin with, the no-load characteristics are compared with FEA predictions as displayed in Figs. 7.10 and 7.11. As observed in both figures, the measured values which reveal some discrepancy when compared to the FEA predictions, peaking to $14.8 \%$ in $2-\mathrm{D}$ and $10.1 \%$ in $3-\mathrm{D}$ at rated field current, is due to a number of design challenges encountered during the fabrication of the prototype. For example, the stack length was affected in the manufacturing process, making it to differ compared to values instituted in FEA. After running short on the supply of the stator lamination modules, due to bad or damaged lamination pieces encountered during the stacking process, the stator stack length of the machine was theoretically reduced by $4 \mathrm{~mm}$ from that originally devised in FEA. This constitutes an estimated $4 \%$ drop in the electromagnetic performance when observed in FEA. 


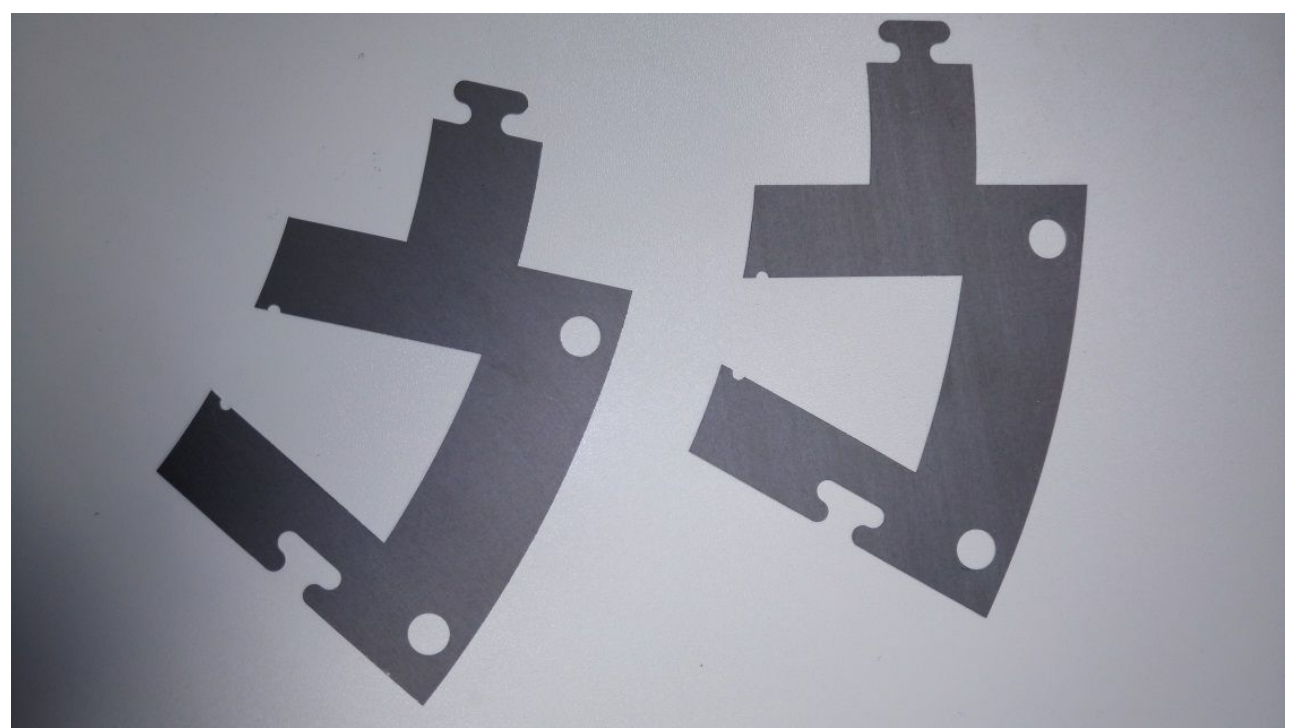

(a)

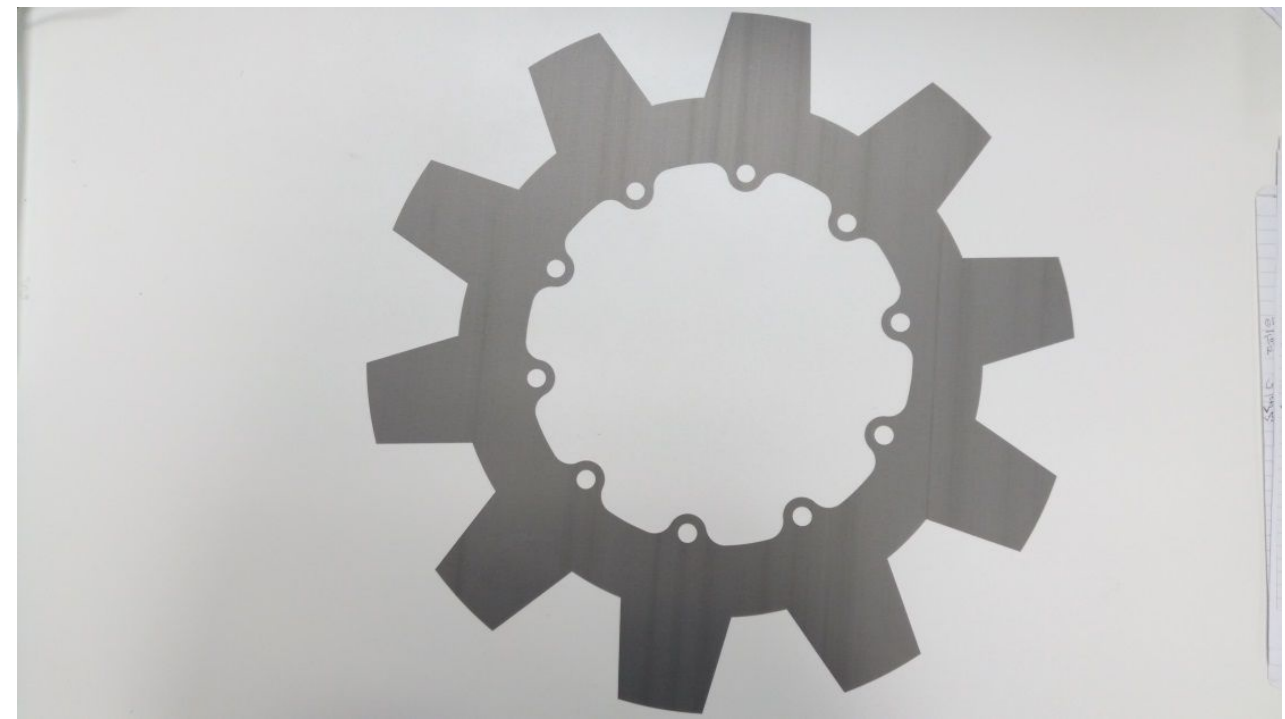

(b)

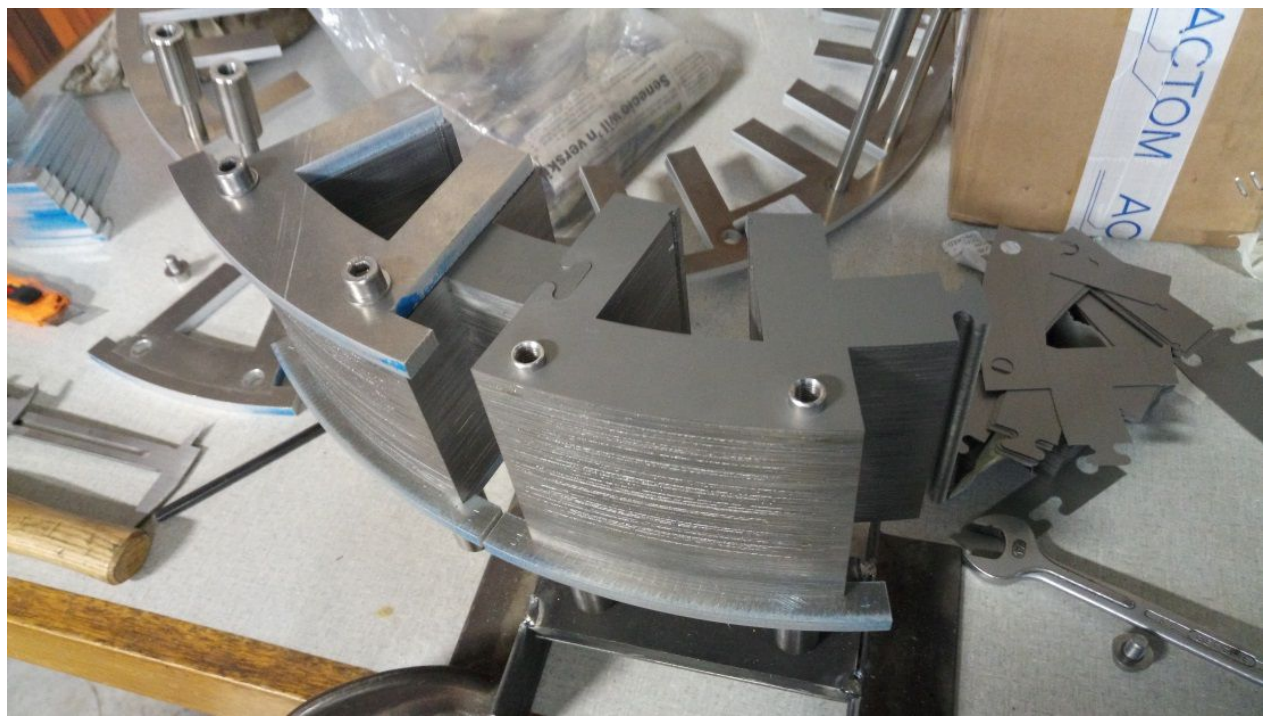

(c)

Fig. 7.4. Manufacturing and assembly: (a) laser cut puzzle-like modular stator lamination units, (b) laser cut simple rotor lamination, and (c) process of stator stack assembling. 


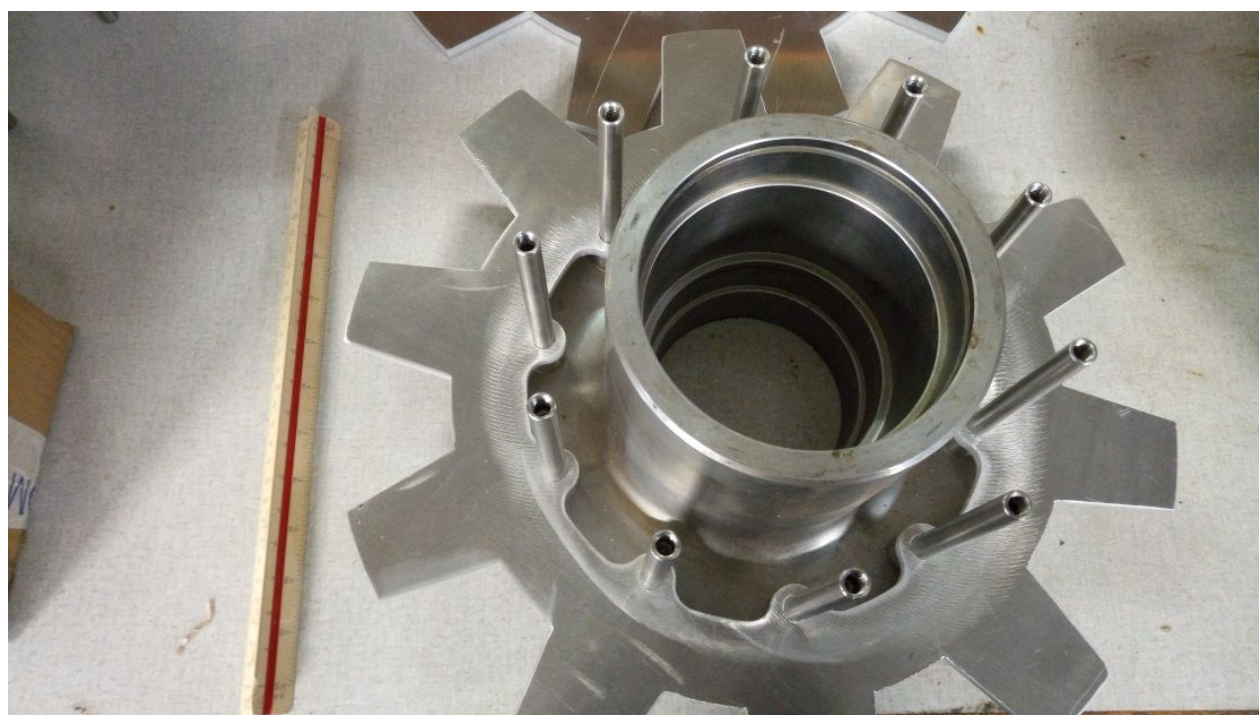

(a)

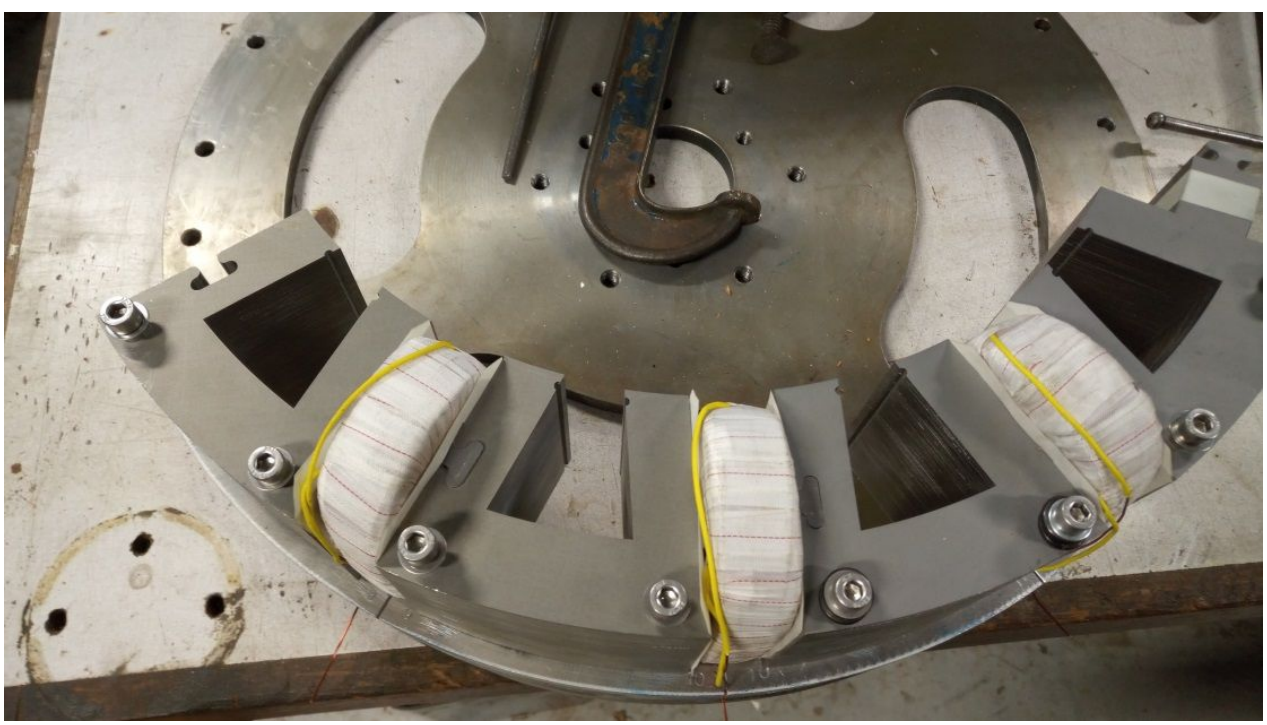

(b)

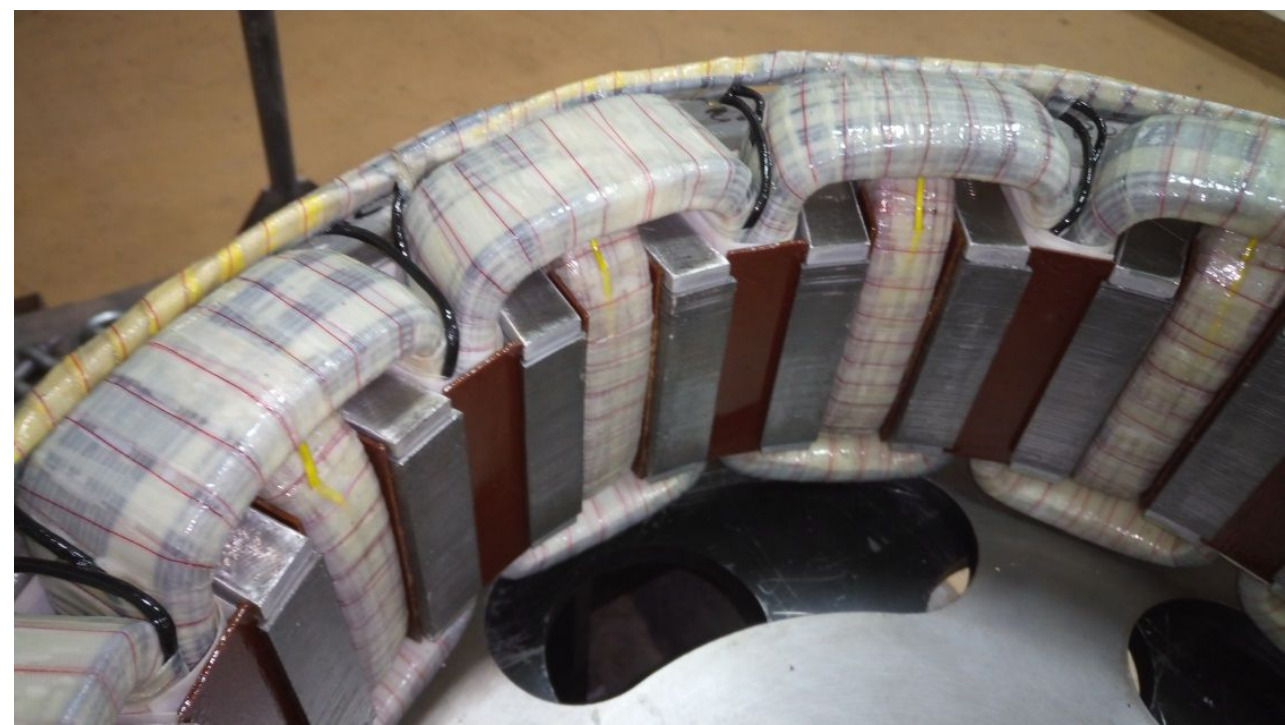

(c)

Fig. 7.5. Manufacturing and assembly: (a) machined rotor hub and rotor guide pin affixed to laser cut end-plate, (b) assembling of wound-fields and stacked stator core, and (c) vanished stator core assembled with wound-fields and phase coils. 


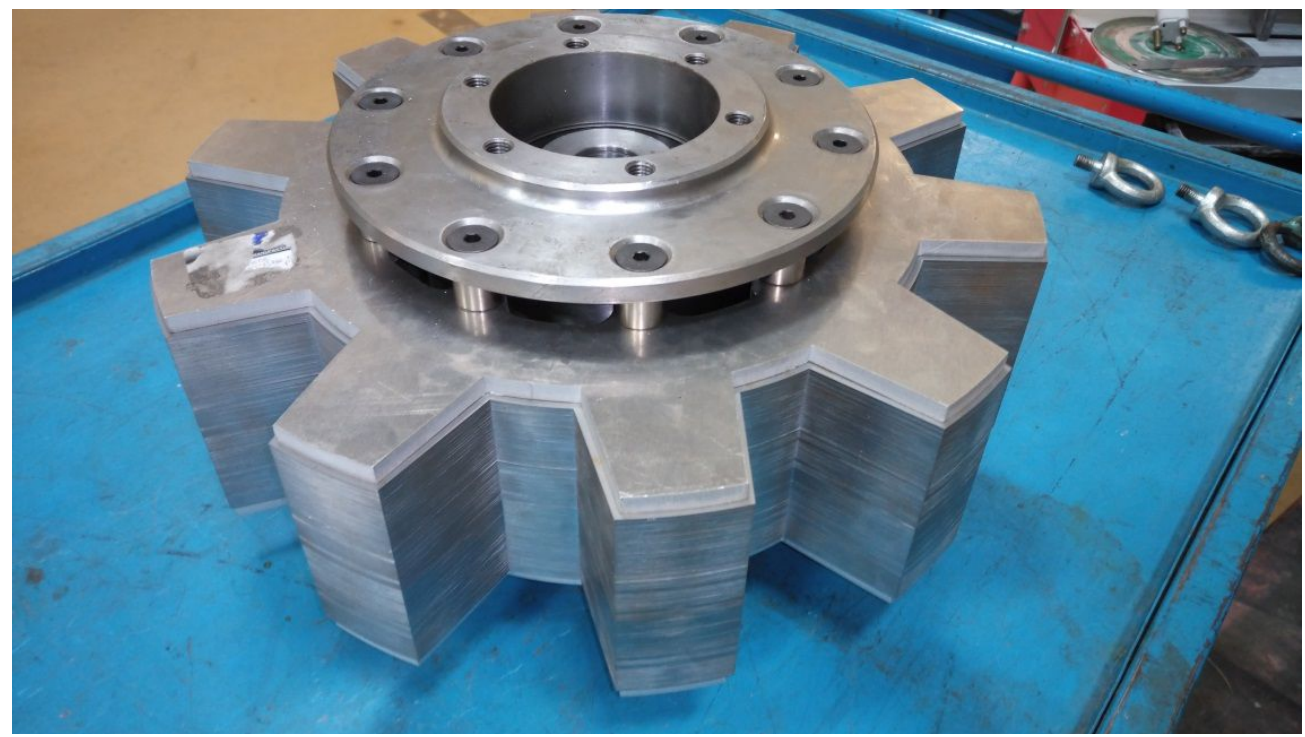

(a)

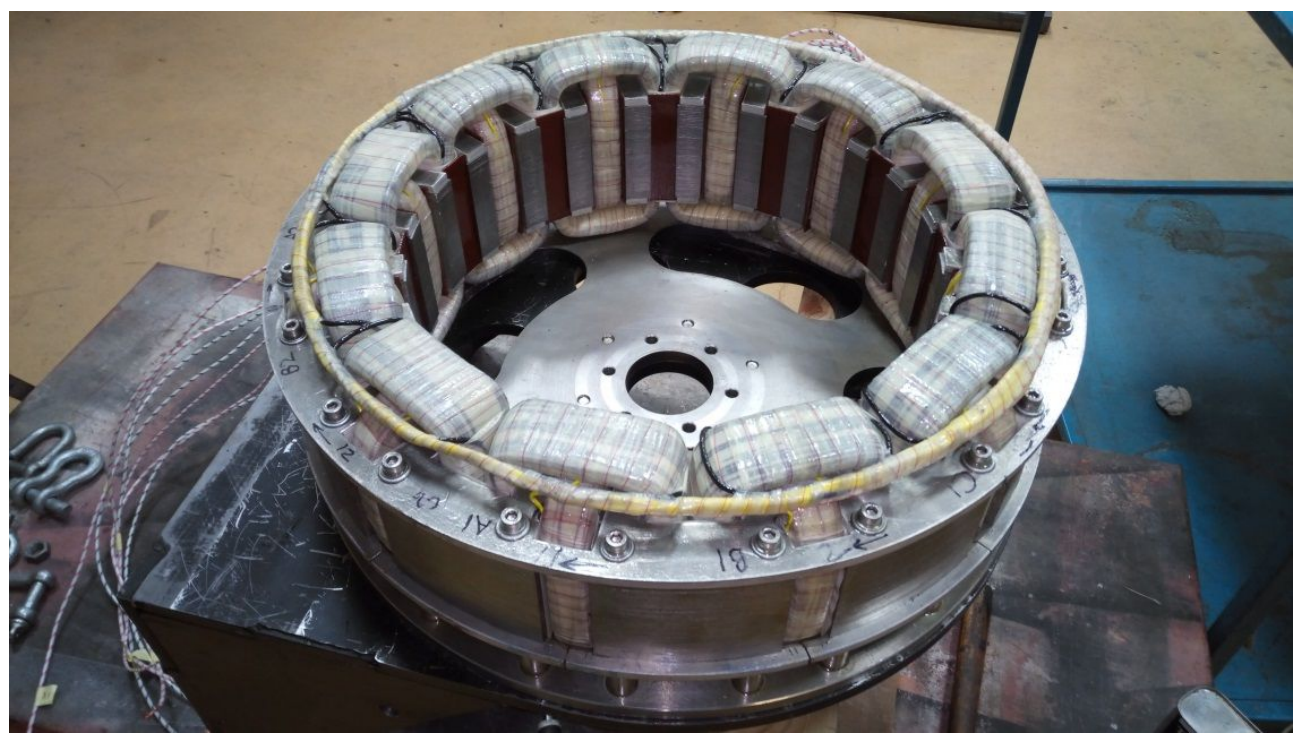

(b)

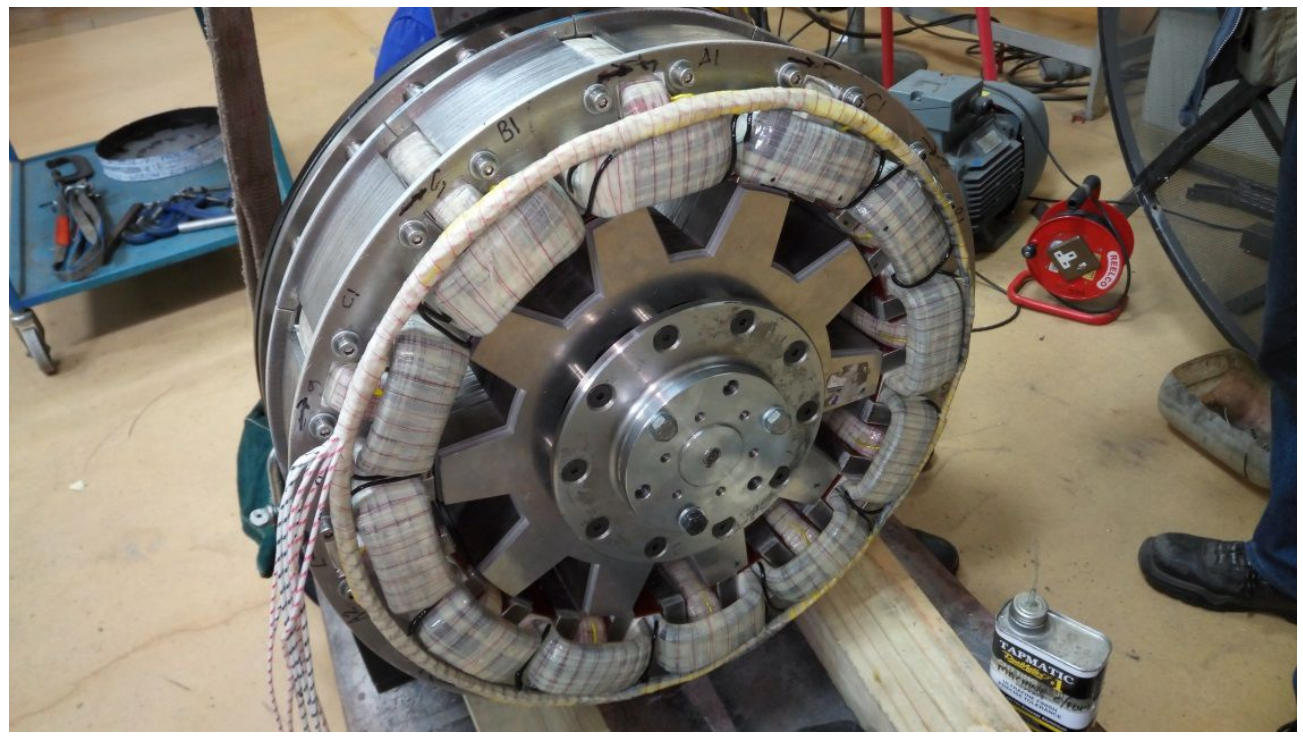

(c)

Fig. 7.6. Finalised assembly: (a) rotor, end plates, bearings and hubs, (b) stator, end plates, coils, back plate and support base, and (c) complete stator and rotor assembly. 


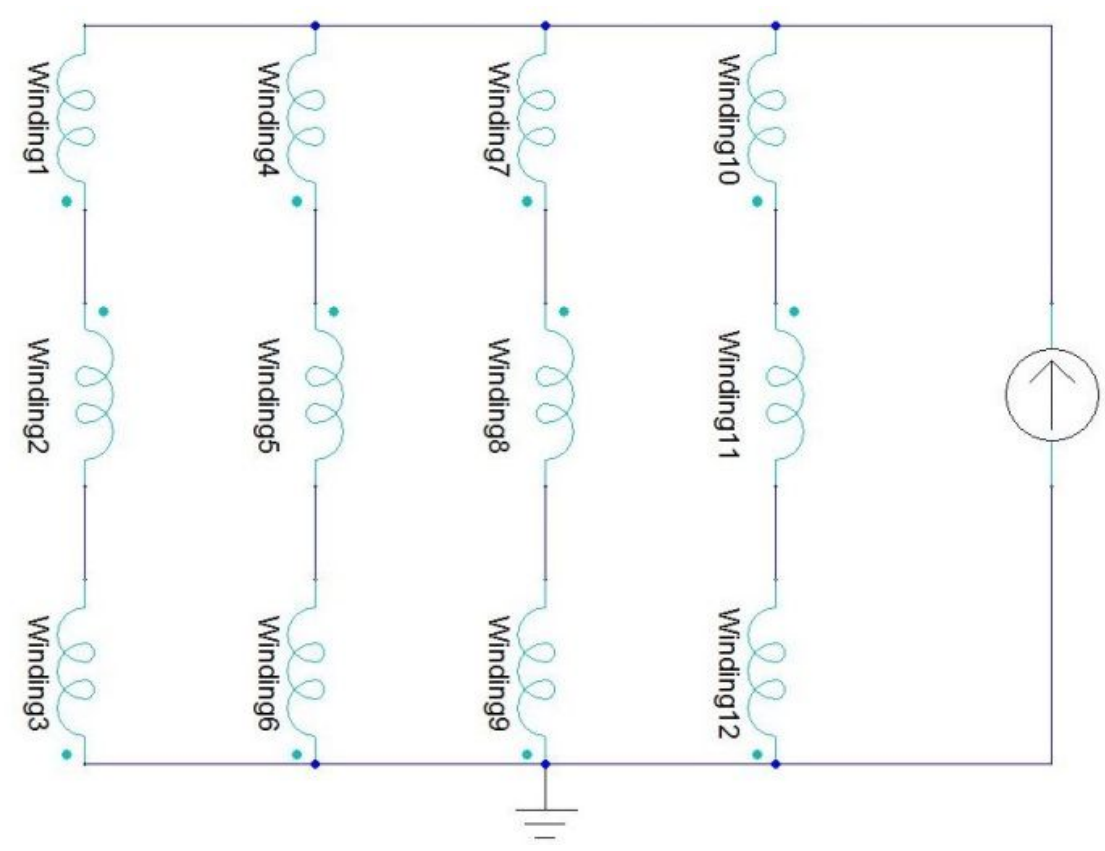

Fig. 7.7. Parallel circuit devised for the DC wound-field coils in the constructed prototype ${ }^{27}$.

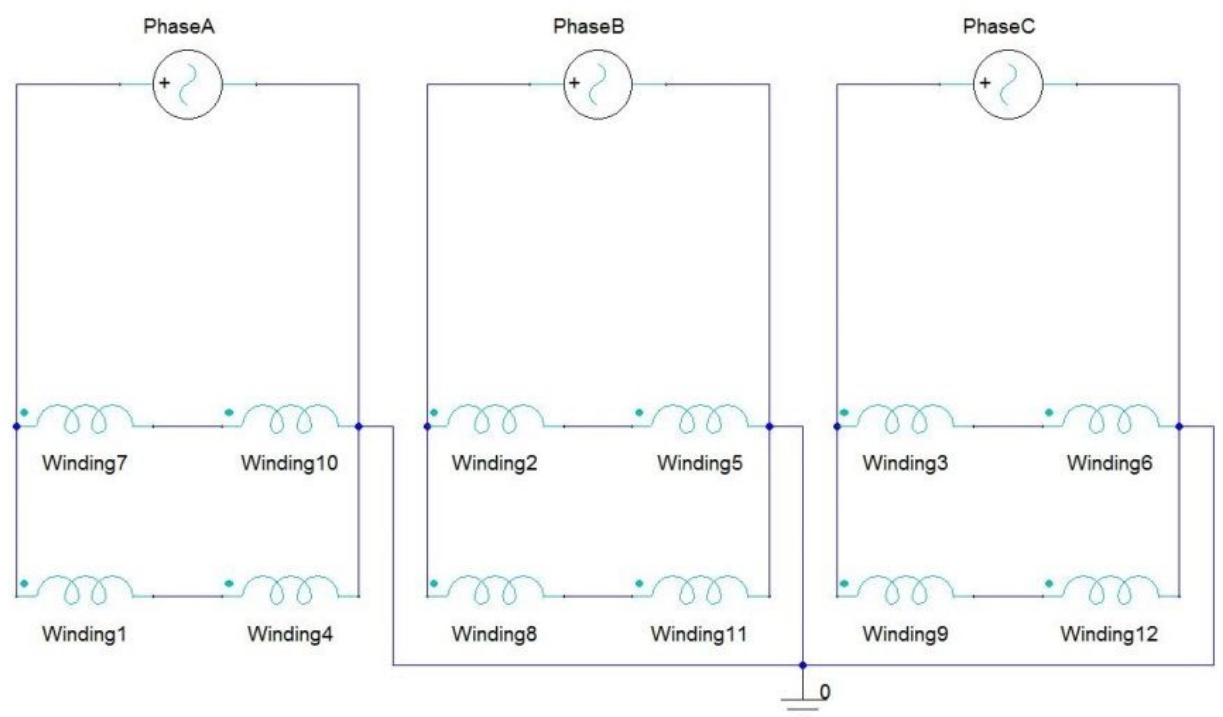

Fig. 7.8. Implemented phase winding layout in the constructed prototype.

Table 7.3. Parallel winding configuration of $10 \mathrm{~kW}$ WF-FSM prototype

\begin{tabular}{c|c|c}
\hline & DC coils & AC coils \\
\hline Number of coils & 12 & 12 \\
Parallel circuits & 4 & 2 \\
Fill factor & 0.45 & 0.45 \\
Winding layers & 1 & 2 \\
Wire diameter $(\mathrm{mm})$ & 1 & 2 \\
Number of turns per coil & 539 & 163 \\
Winding resistance per coil $(\Omega)$ & 5.585 & 0.447 \\
Coil area $\left(\mathrm{mm}^{2}\right)$ & 423.507 & 512.720 \\
\hline
\end{tabular}

27 It is imagined that the total reactance and reactance are embedded for each of the winding represented; the same assumption is uplifted in Fig 7.8 which follows. 


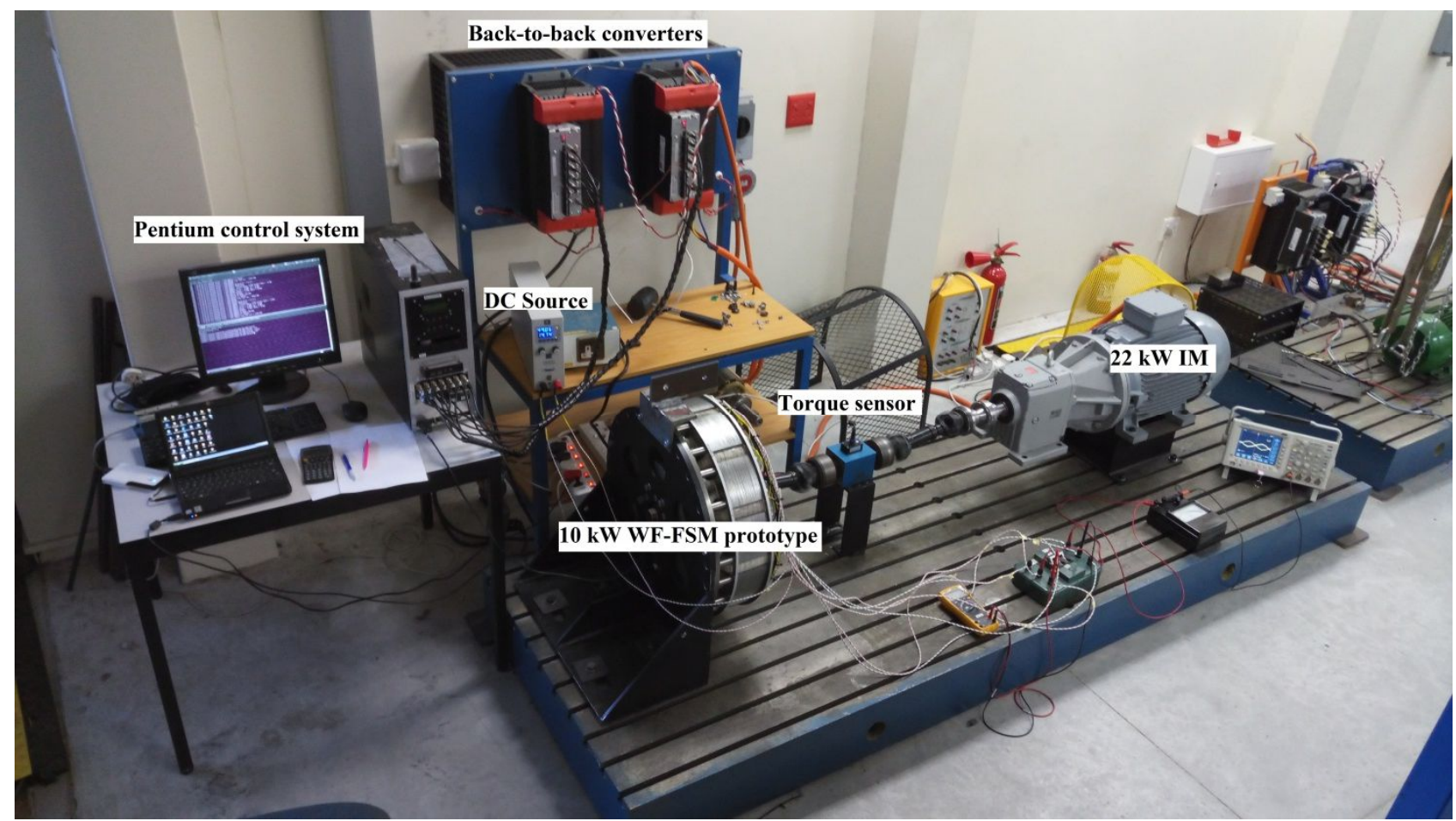

Fig. 7.9. Experimental test-bench used for actualising measurements on the constructed prototype.

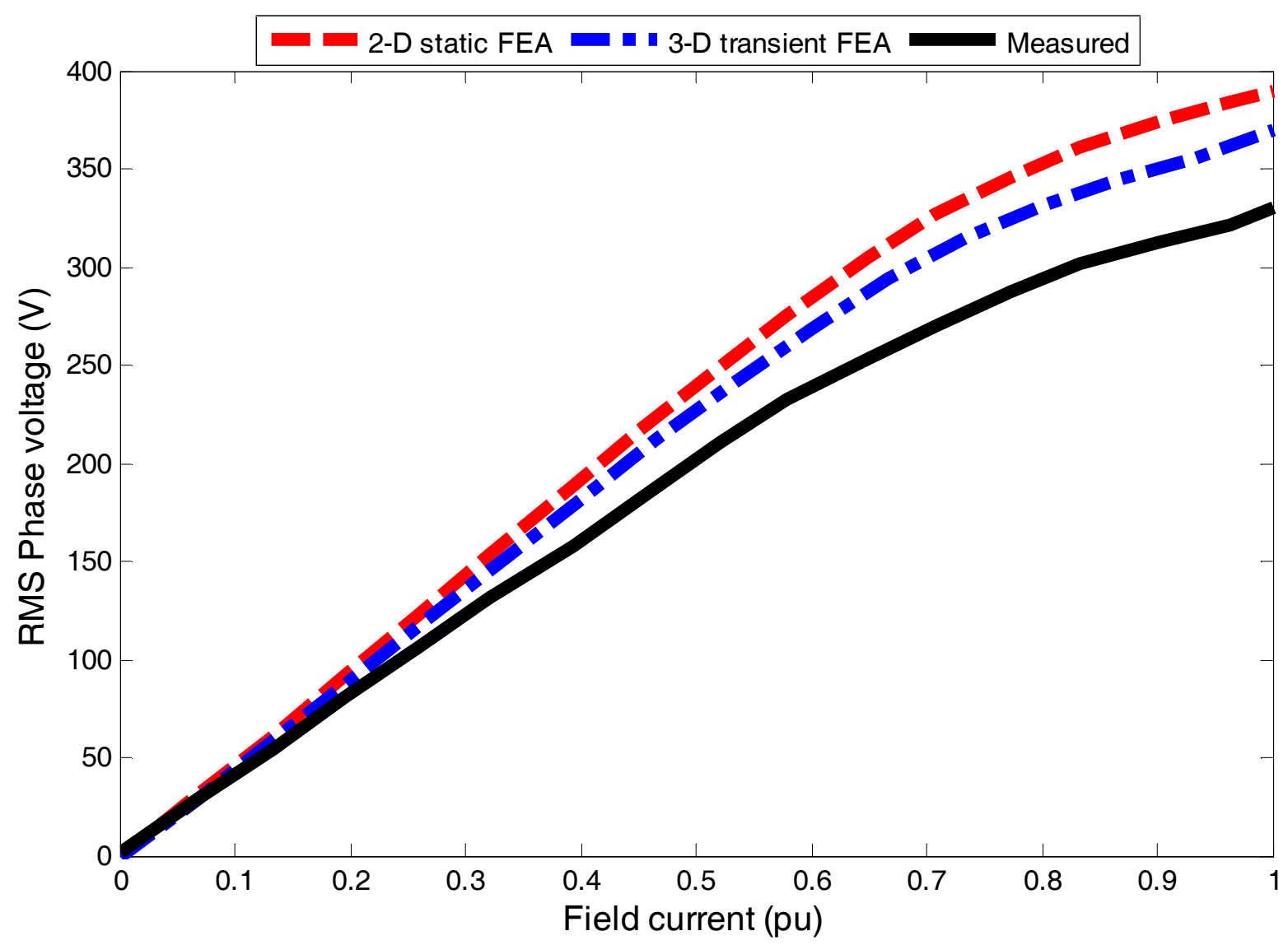

Fig. 7.10. No-load curve at $360 \mathrm{r} / \mathrm{min}$ (base value $=$ rated field current). 


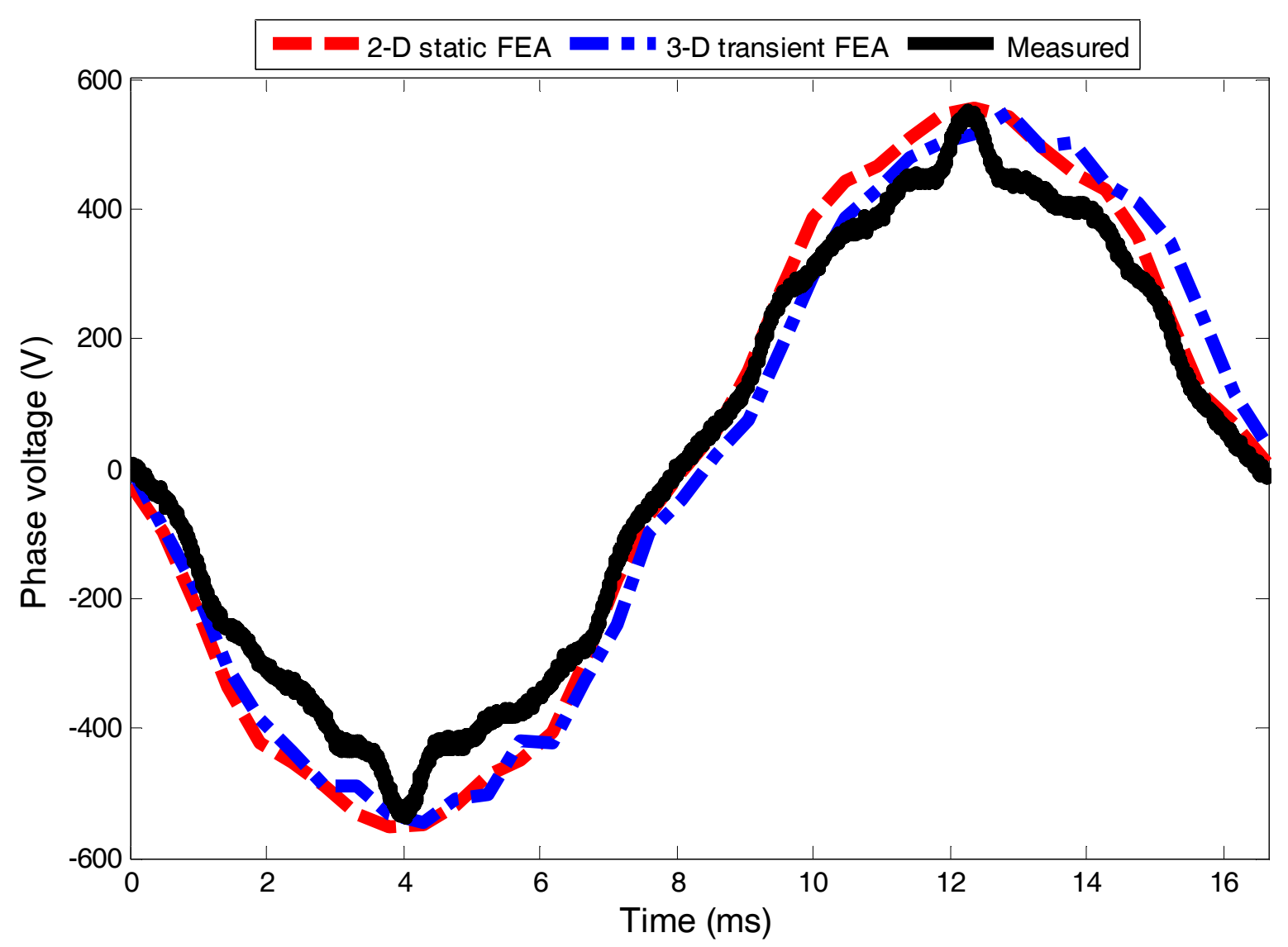

Fig. 7.11. No-load voltage waveforms at rated field current and $360 \mathrm{r} / \mathrm{min}$.

Another reason for the observed discrepancy in the measured and predicted no-load phase voltages is due to unaccounted leakages in the 2-D and 3-D FEA calculations, later escalated in subsection 7.3.3 for the on-load characterisation. The leakages are also due to the fringing effects along the axial paths which are not classified in 2-D FEA solutions as demonstrated in Fig. 6.7 in Chapter 6.

As for the no-load voltage waveforms shown in Fig. 7.11, the representation of sinusoidal waveforms is exhibited to clearly establish the AC drive capability of the manufactured WF-FSM prototype in contrast with FEA modelling. Observe that the waveform trajectory of the measured noload output voltage is properly trained in line with the FEA predictions. Further revelations reveal prominent spikes in the measured no-load voltage waveform compared to FEA. This observation can be attributed to an irregular airgap length conceived in the composed prototype as a result of the minor issues encountered in assembling the modularised stator stacks. Also, it can be attributed to the fact that the wound-fields exhibit tendencies towards inducing non-DC voltage components in their windings due to mutual coupling with the time varying flux linkages of the phase windings, which are not initially compensated, especially in the 2-D static FEA predictions.

Further tests as performed under no-load conditions are compiled as shown in Table 7.4. The 
results are slightly good in agreement, with such exceptions on the core loss-elaborated in the accompanying footnote. Note that, the winding resistances are approximated experimentally when only DC voltage is applied to the terminals of the windings in order to suppress their reactances.

\subsubsection{Short-Circuit Characteristics, Heat and Resistance Tests}

The circuit propagated for the short-circuit test is as shown in Fig. 7.12. The obtained shortcircuit characteristics and waveforms at rated field current is compared with 2-D FEA and displayed as shown in Figs. 7.13 and 7.14. Due to the unsaturated operating condition during short-circuit tests compared to open-circuit conditions where higher saturation is observed at higher field currents (see Figs. 7.10 and 7.11); a better confidence is herein established.

Besides, while operating at rated short-circuit, heat runs in the wound-field and phase winding coils were conducted using a temperature camera in order to evaluate their steady-state thermal effects. Three different hotspots are identified in the coils and considered as shown in Fig. 7.15, while a sample of the steady-state readings as obtained after running the machine for 90 minutes is shown in Fig. 7.16. Note that, there has been no elaborate cooling measure implemented on the prototype, no thanks to the fact that the machine is not enclosed in any casing.

Table 7.4. Resistance and core loss tests evaluated at no-load (360 r/min)

\begin{tabular}{c|c|c|c}
\hline & 2-D FEA & 3-D FEA & Measured \\
\hline \hline Phase resistance, $R_{S}{ }^{28}(\Omega)$ & 0.47 & 0.49 & 0.53 \\
Wound-field resistance, $R_{F}(\Omega)$ & 4.18 & 3.51 & 3.08 \\
Core loss, $P_{\text {Core }}(\mathrm{W})$ & 199.00 & 211.40 & $249.30^{29}$ \\
\hline
\end{tabular}

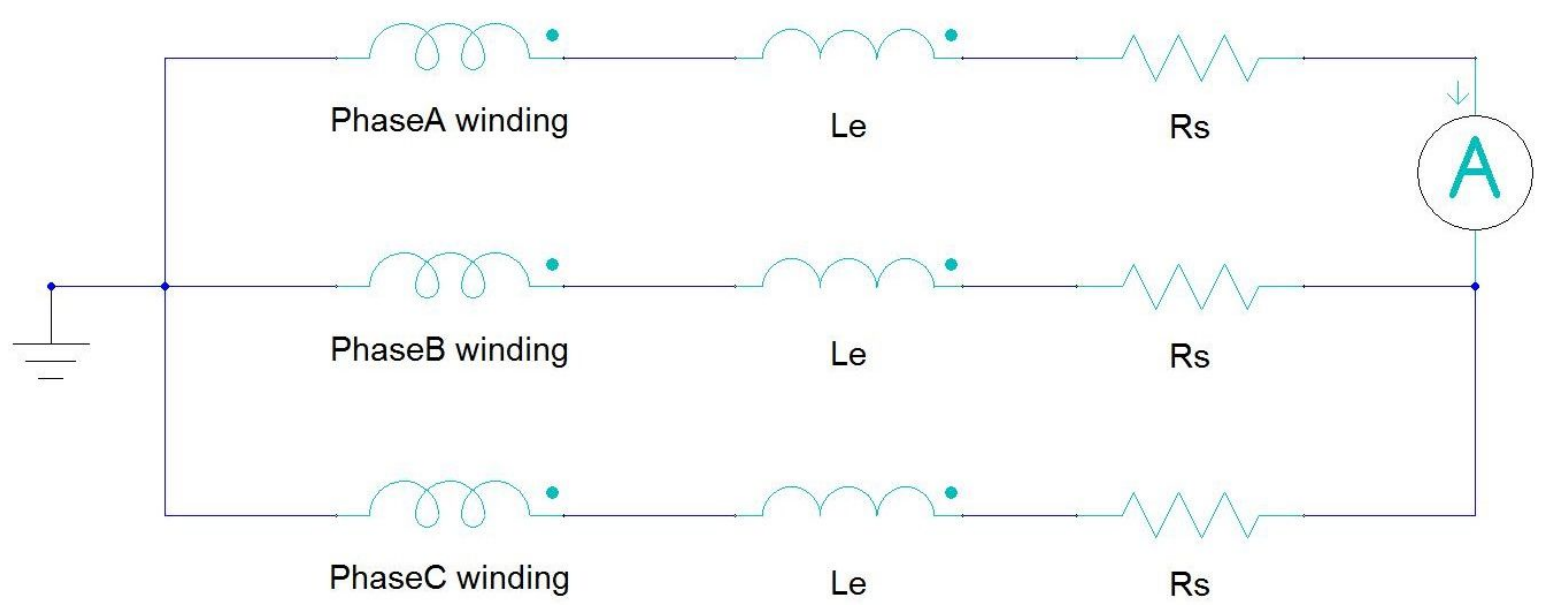

Fig. 7.12. Implementation of the Wye-connected three-phase short-circuits.

28 It is estimated as the effective value seen after the output coil terminals of the parallel circuits implemented, same for the field resistance $\left(R_{F}\right)$.

29 The total no-load input power evaluated as the no-load rotational loss and calculated with the assistance of the torque sensor shown in Fig. 7.6, usually includes the core losses, as well as friction and windage losses. Thus, in the event that the latter losses are explicit, the measured core loss value should be less than that reported. 


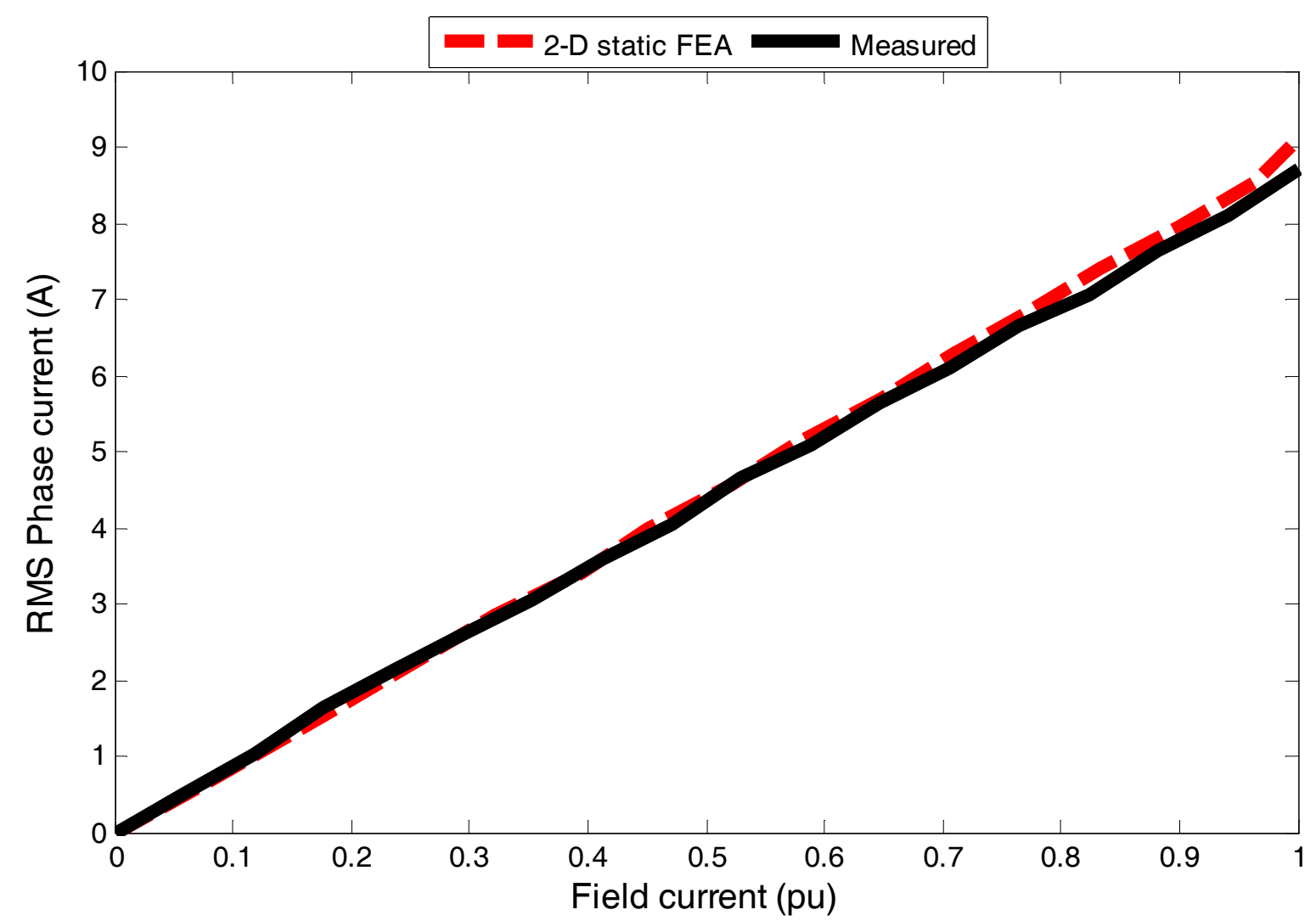

Fig. 7.13. Short-circuit characteristics of the $10 \mathrm{~kW}$ WF-FSM prototype operated at $360 \mathrm{r} / \mathrm{min}$ (base value $=$ rated field current).

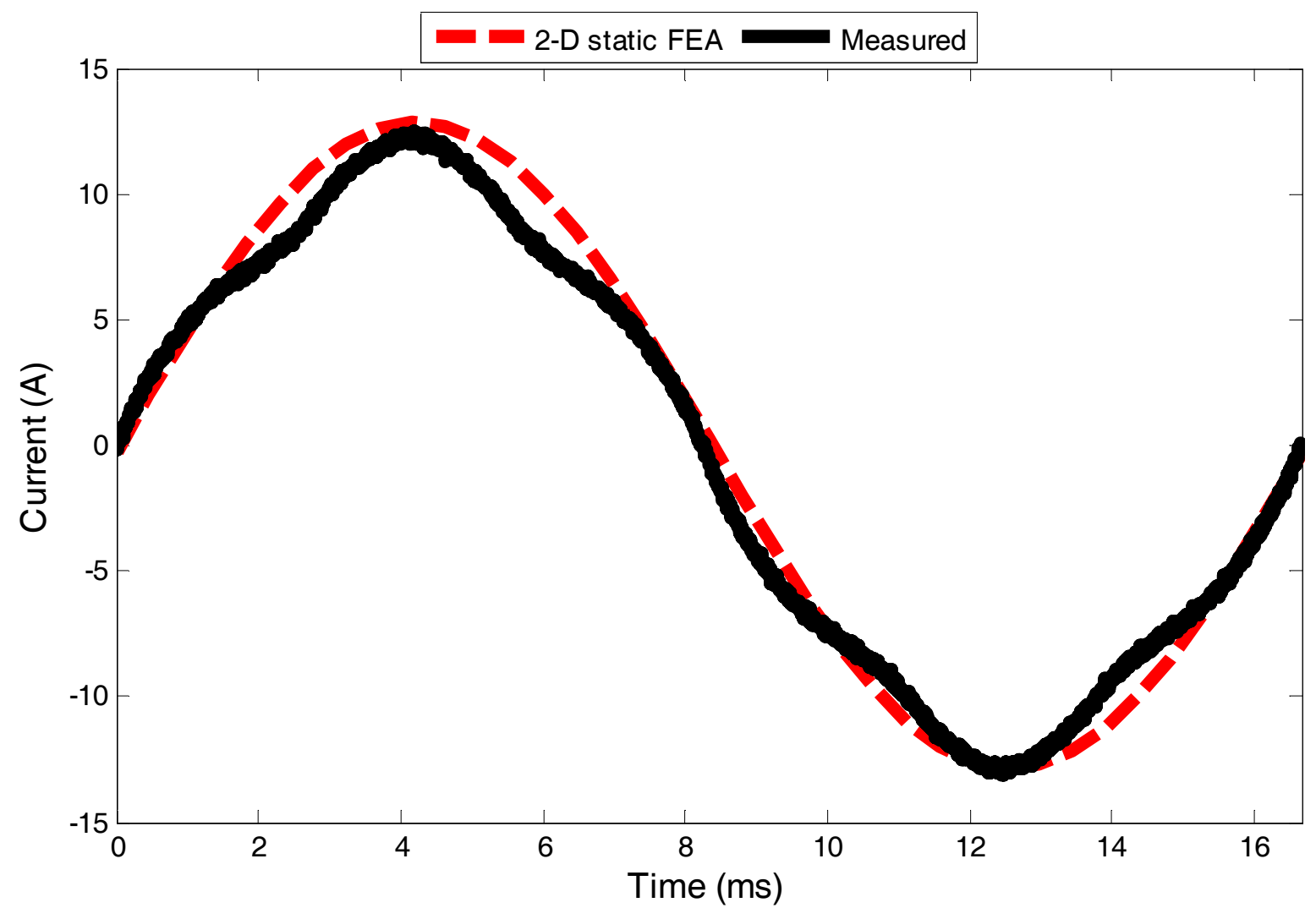

Fig. 7.14. Short-circuit current waveforms of the $10 \mathrm{~kW}$ WF-FSM prototype operated at rated field current and $360 \mathrm{r} / \mathrm{min}$. 


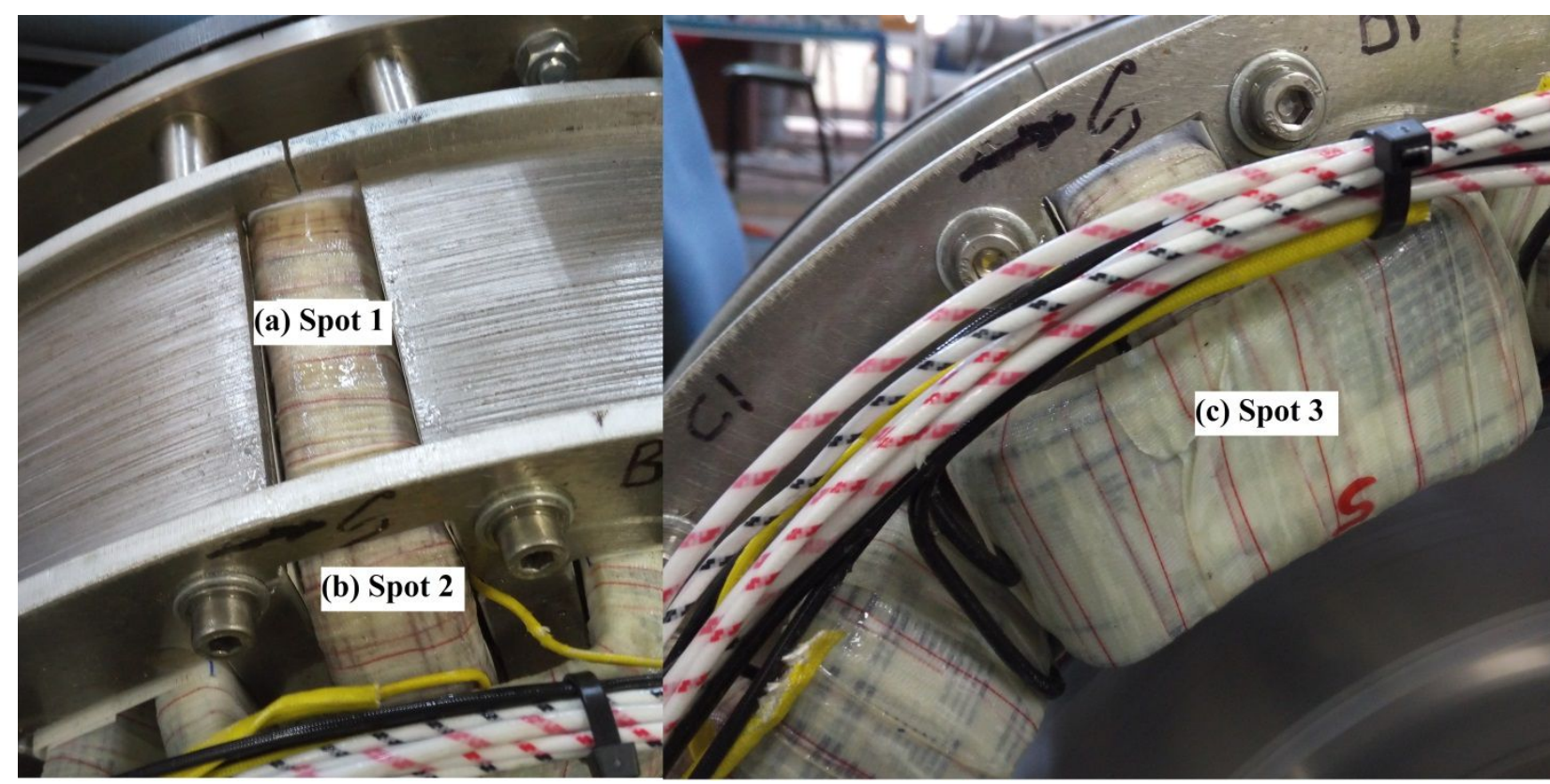

Fig. 7.15. Representation of the different temperature hotspots: (a) Top projection on DC coil along the axial path, (b) end-winding knee on DC coil, and (c) top projection on AC coil on the radial side.

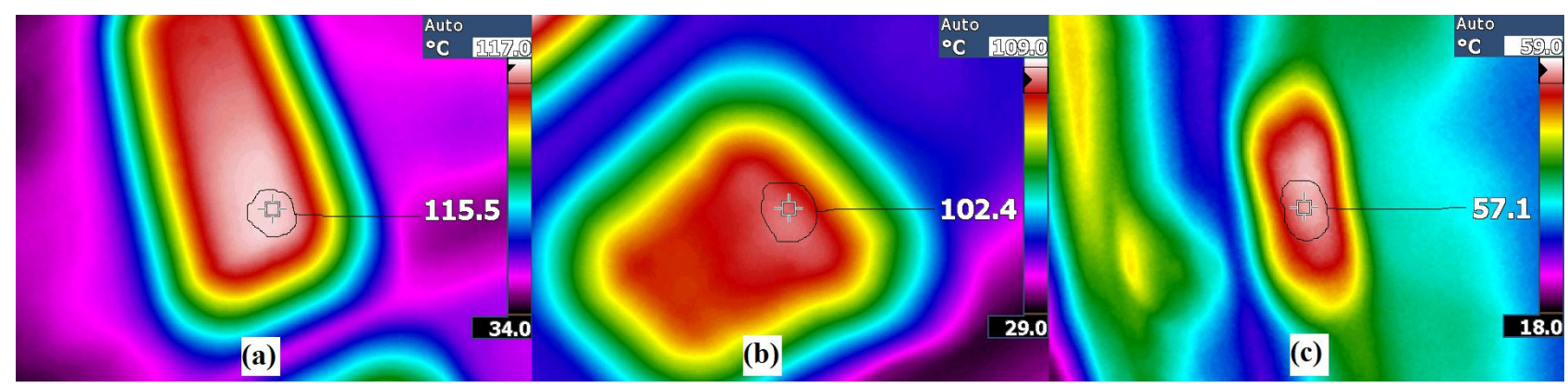

Fig. 7.16. Contour maps of instantaneous temperature readings observed after 90 minutes in the different hotspots: (a) Spot 1, (b) Spot 2, and (c) Spot 3.

Meanwhile, the temperature buildups studied in the highlighted hotspots are reported in Fig. 7.17. The observed temperature rise after 90 minutes under short-circuit condition is $75{ }^{\circ} \mathrm{C}, 78{ }^{\circ} \mathrm{C}$ and $35^{\circ} \mathrm{C}$ in hotspots 1,2 and 3, respectively. This implies that the DC coils' of the constructed prototype heat up very rapidly, and the knee points on its end-winding can heat up even faster, compared to that of the phase windings. The reason for this is likely because of a higher current density predicted in the DC coils at $\sim 5 \mathrm{~A} / \mathrm{mm}^{2}$, with that of the phase windings at $1.64 \mathrm{~A} / \mathrm{mm}^{2}$. However, by visual inspection of the measured current displayed during the short-circuit experimentation; it is revealed that the operation of the machine is not impacted by the rapid temperature rise in the DC coils. Obviously, there is absolutely no fear of demagnetisation in the WF-FSM prototype, and as shown in Fig. 7.18, it is clearly seen that the composed stator laminations is also not particularly encumbered by the thermal activities in the coils. 


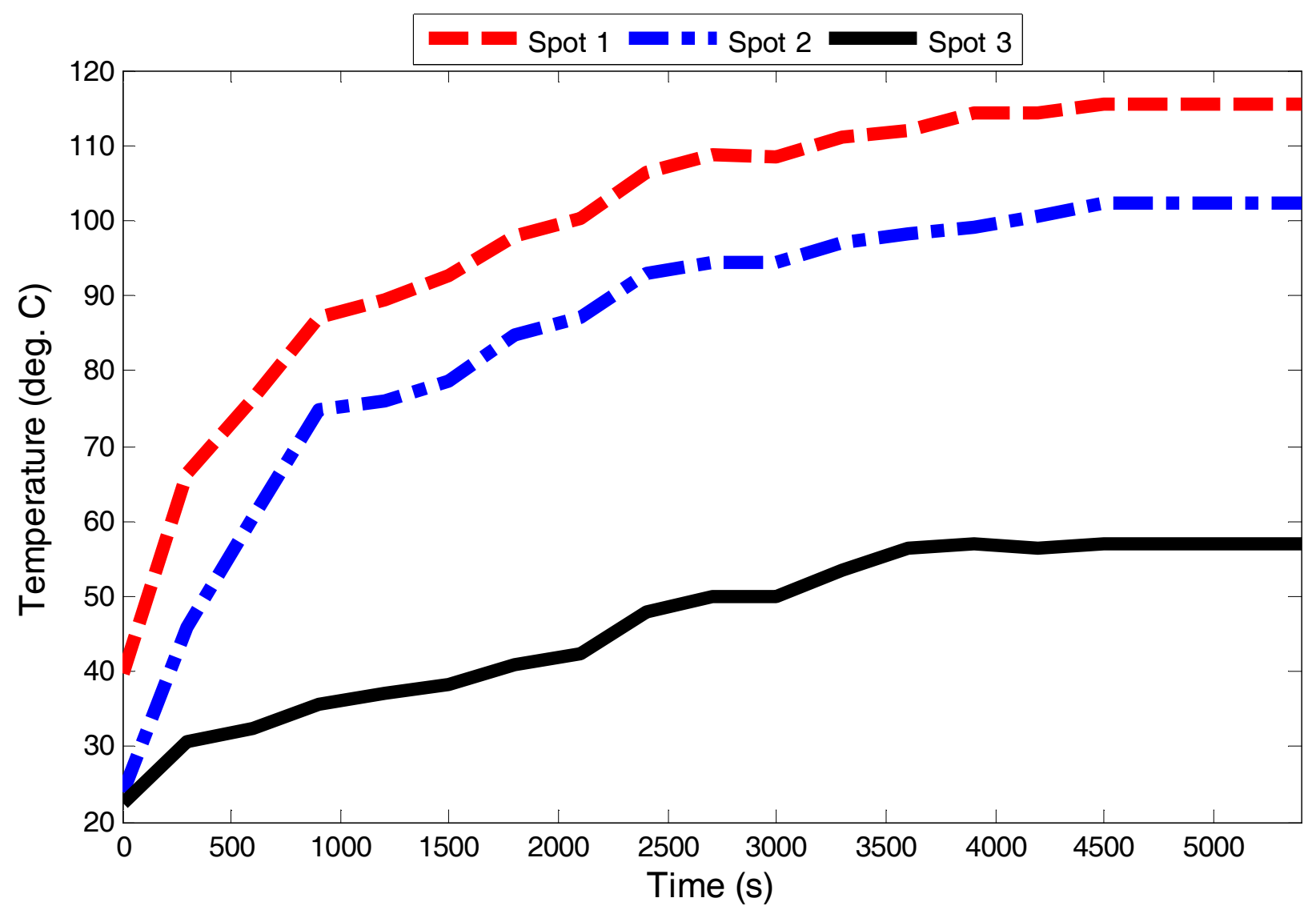

Fig. 7.17. Patterns of temperature rise during short-circuit operation in the major hotspots of the 10 $\mathrm{kW}$ WF-FSM prototype when operated at rated field current and $360 \mathrm{r} / \mathrm{min}$.

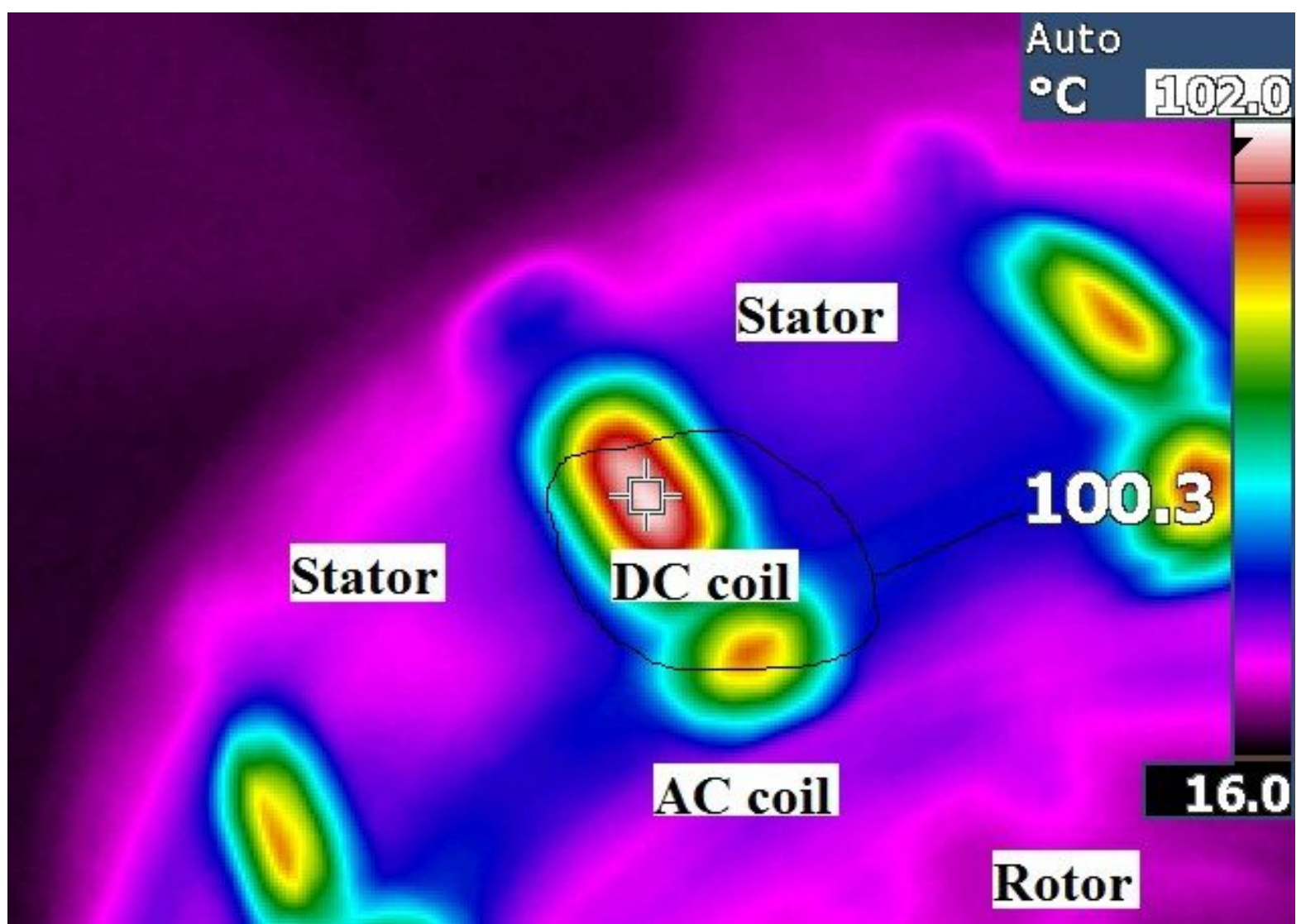

Fig. 7.18. Snapshot of very low-profile thermal activity in the surrounding stator lamination stack. 
Furthermore, a three-phase Wye-connected resistive load of 0.8 Ohms per phase is implemented on the experimental prototype as shown in Fig. 7.19. The obtained current and voltage waveforms for the connected load resistance, which is just about the same value as the measured phase resistance of the armature windings, are as shown in Fig. 7.20, with RMS phase values of 8.11 A and $5.69 \mathrm{~V}$, respectively. An overload resistance test operated at the rated field current is also performed, whereby the load resistance is adjusted upwards to $10 \mathrm{Ohms}$ per phase, which is almost 20 times that of the generator phase resistance. To this end, the machine can safely run for about 10 minutes without any harm recorded, clearly confirming the overload and thermal capability in safely operating the machine at higher temperatures. Meanwhile, the obtained voltage and current waveforms of the 10 Ohms load is equally shown in Fig. 7.21, with the RMS phase current and voltage measured at 7.02 A and $95.6 \mathrm{~V}$, respectively.

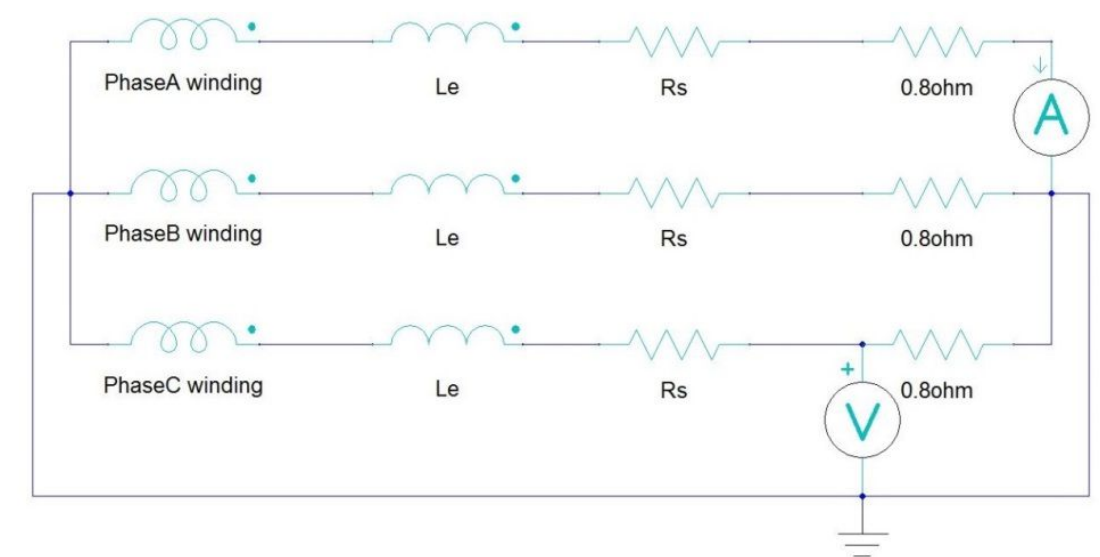

Fig. 7.19. Load resistance connection in uncontrolled load tests.

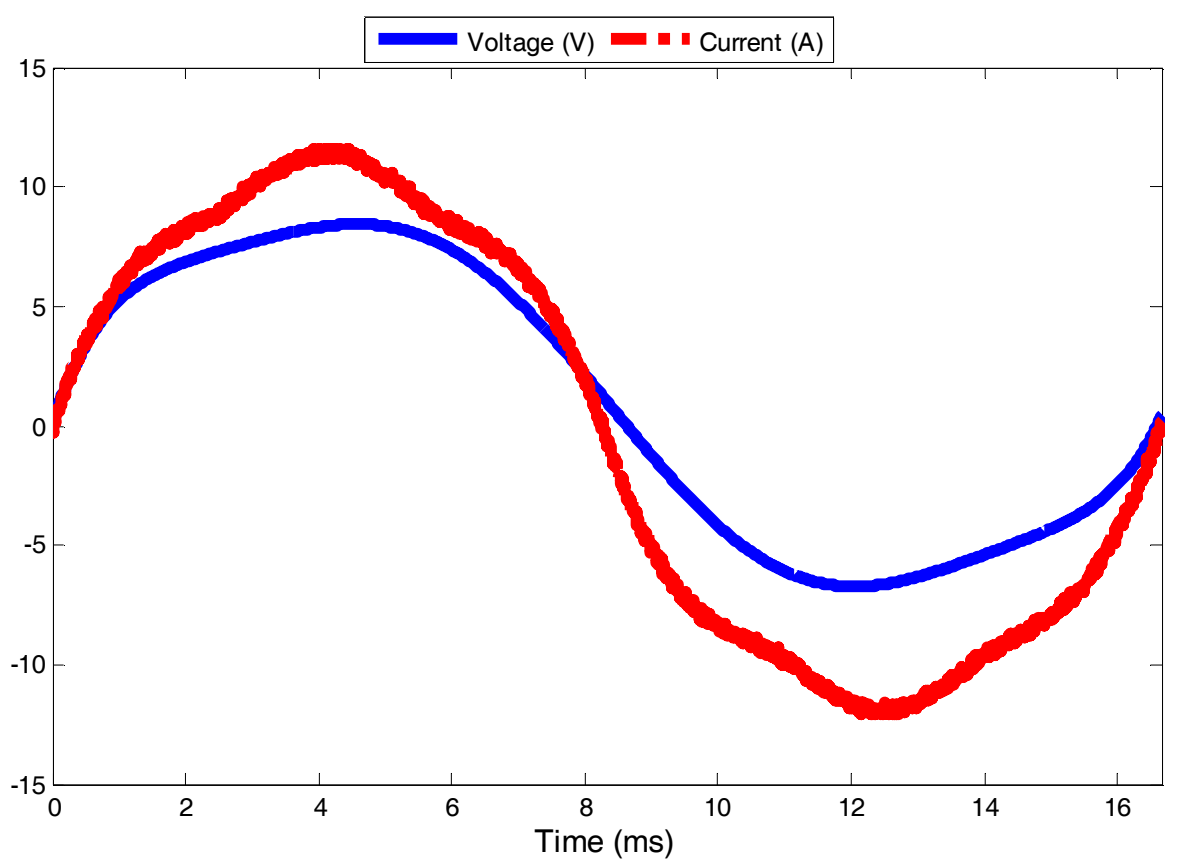

Fig. 7.20. Obtained current and voltage waveforms of the $10 \mathrm{~kW}$ WF-FSM prototype under load of $0.8 \mathrm{Ohms}$ per phase operated without field-oriented control at rated field current and $360 \mathrm{r} / \mathrm{min}$. 


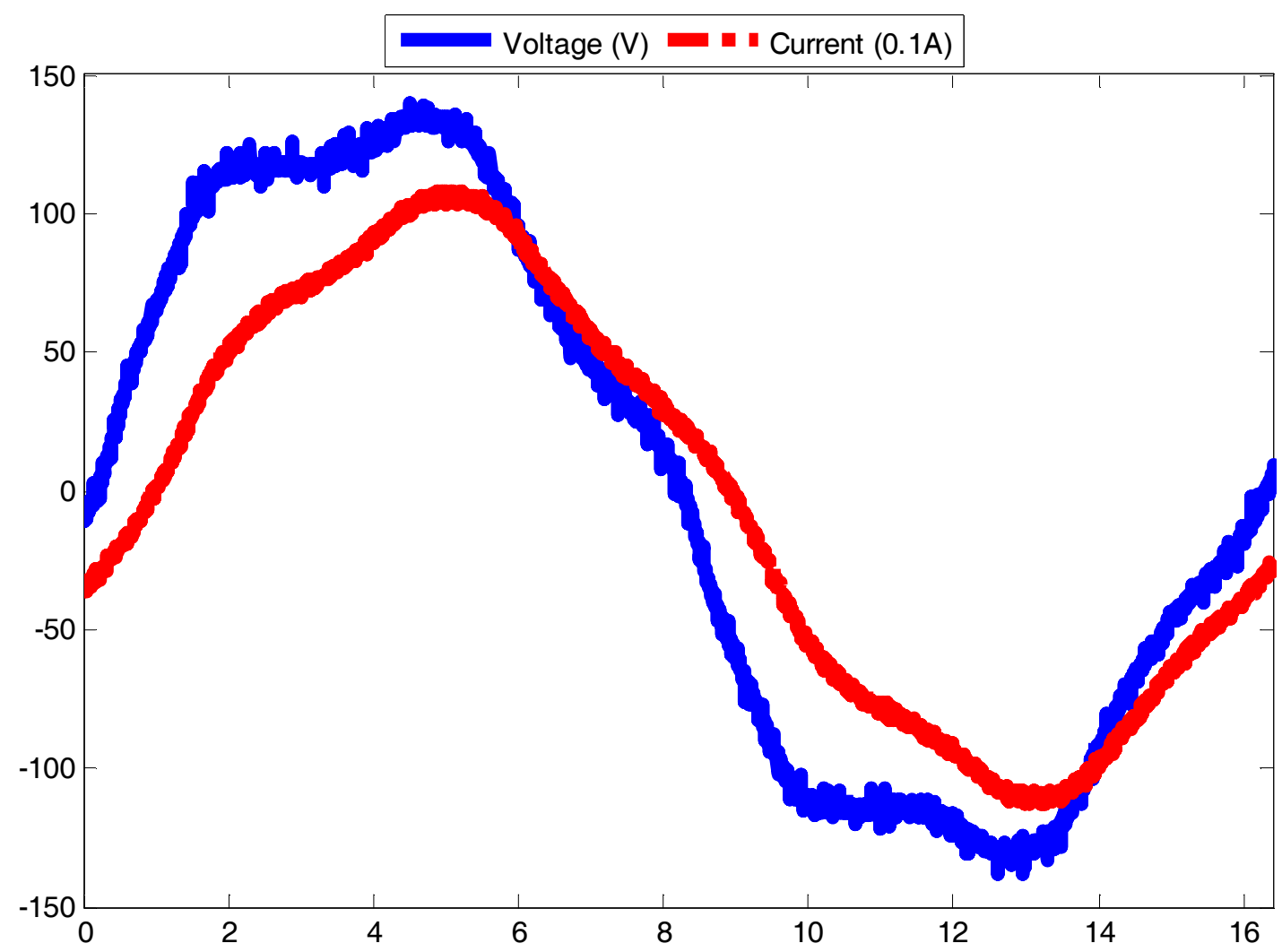

Fig. 7.21. Current and voltage waveforms of the $10 \mathrm{~kW}$ WF-FSM prototype under uncontrolledoverload (10 Ohms per phase) operating condition at rated field current and $360 \mathrm{r} / \mathrm{min}$.

\subsubsection{Controlled Generator Mode and Voltage Regulation}

It is important to understand that without the vector control of the magnetic axes of the phase $A$ currents to align with the rotor magnetising fields, it is impossible to obtain the reference electromagnetic torque required for the propagation of full electrical power in the manufactured prototype. Such is the case with FSMs, even as their behaviour and control are similar to conventional synchronous machines, according to Cheng et al (2011) [44], Shen and Fei (2013) [141] and Sikder, Iqbal and Ouyang (2016) [142]. Thus, in this subsection, a complete drive system is implemented to study the performance of the manufactured machine in its proposed generator mode. However, note that only the half of the rated speed command is adopted during the tests due to limitations imposed by the reference voltage of the inverter.

Consequently, stator field-oriented control with the aid of a speed encoder is adopted in order to align the apparent $\mathrm{d}$-axis of the rotor with the magnetic axis of the stator phase $A$ winding current as captured in Fig. 7.22, which shows the frozen experimental signals of the steady-state data while operating at approximately half-rated speed $(180 \mathrm{r} / \mathrm{min})$ and at rated field and RMS currents. It can be observed that the phase current lags the displayed PWM inverter reference voltage, an indication that the terminal voltage would slightly increase from no-load to rated conditions. 


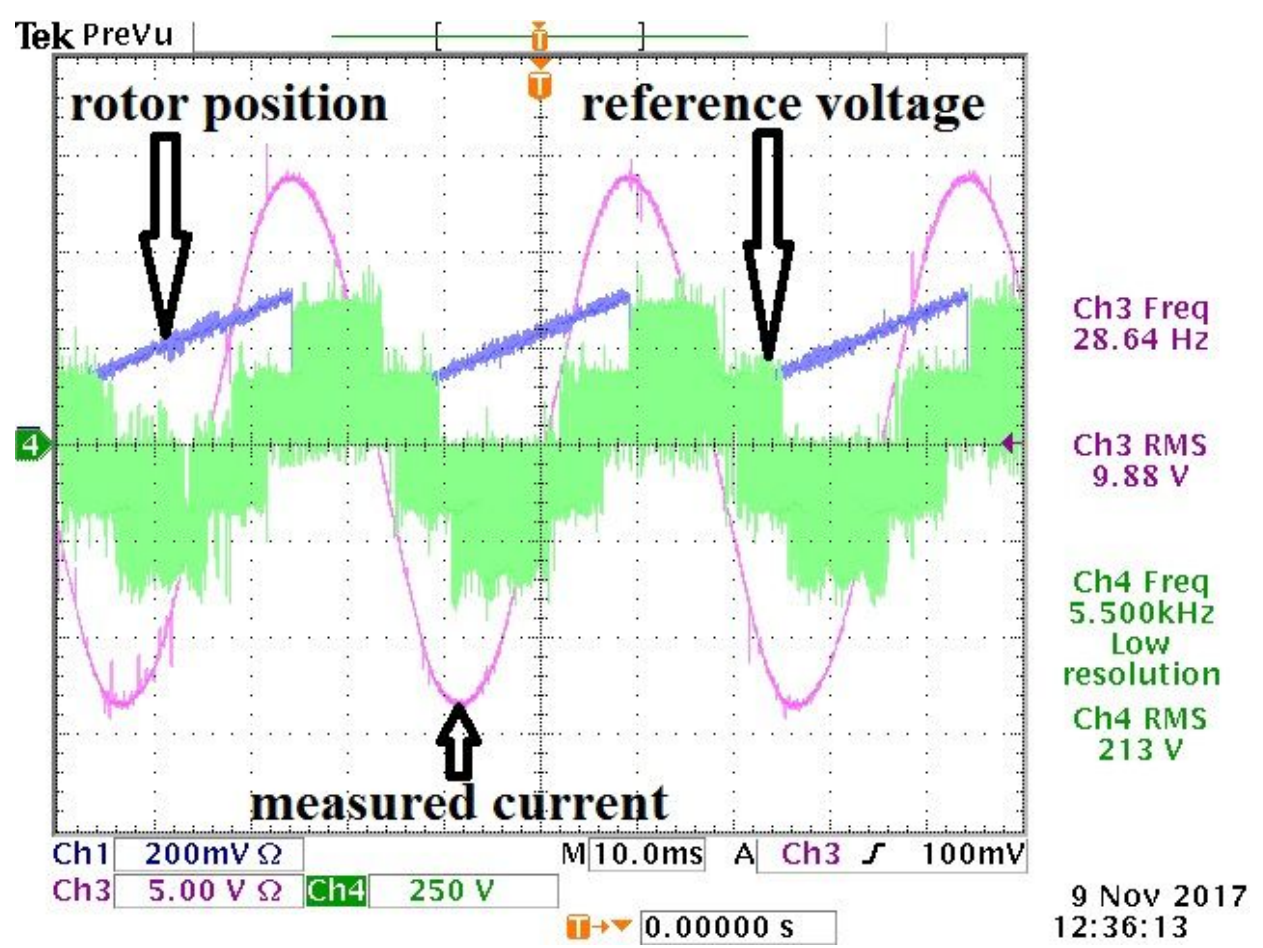

Fig. 7.22. Sample data measured on oscilloscope showing rotor alignment at $\sim 180 \mathrm{r} / \mathrm{min}$ and rated field and phase currents.

Figs. 7.23-7.25 show the comparison of the 2-D static, 3-D transient FEA predicted results and measured experimental values for certain generator performance quantities, based on phase current profiles from no-load to full-load, at rated field current and half-rated speed. In terms of the induced voltages and average torque values, the 3-D FEA prediction displays good agreement with that of the measured results, especially at lower currents. The observed increasing discrepancy as the current increases is due to a number of reasons. One, it is already mentioned in subsection 7.3.1 where no-load tests were conducted that certain structural imperfections accumulated during the fabrication process is partially culpable for such discrepancies. Two, due to the small aspect ratio of the constructed machine and a purported underestimation of the effect of end leakages, the phase winding inductance increases as the phase current rises, which results in rapid saturation and impermeability of the laminated iron cores. The impact of the end-winding leakages of the coils is one of such de facto causes. As a matter of fact, the same assertion is responsible for the discrepancy observed between the predicted and measured values of the power factor displayed in Fig. 7.27. Based on (2.5) and (2.16) in Chapter 2, it is clear to infer that increase in saturation decreases the magnetising inductance, viz., the power factor.

With regards to the 2-D FEA results facilitated by dq-modelling, the higher deviations in Figs. 7.23 and 7.24 primarily result from the implemented static solutions in not making any attempt to account for a complex non-linear magnetic coupling effects of the field and phase winding currents, Zulu, Mecrow and Armstrong: 2010 [147] and Balyovski et al: 2014 [148]. 


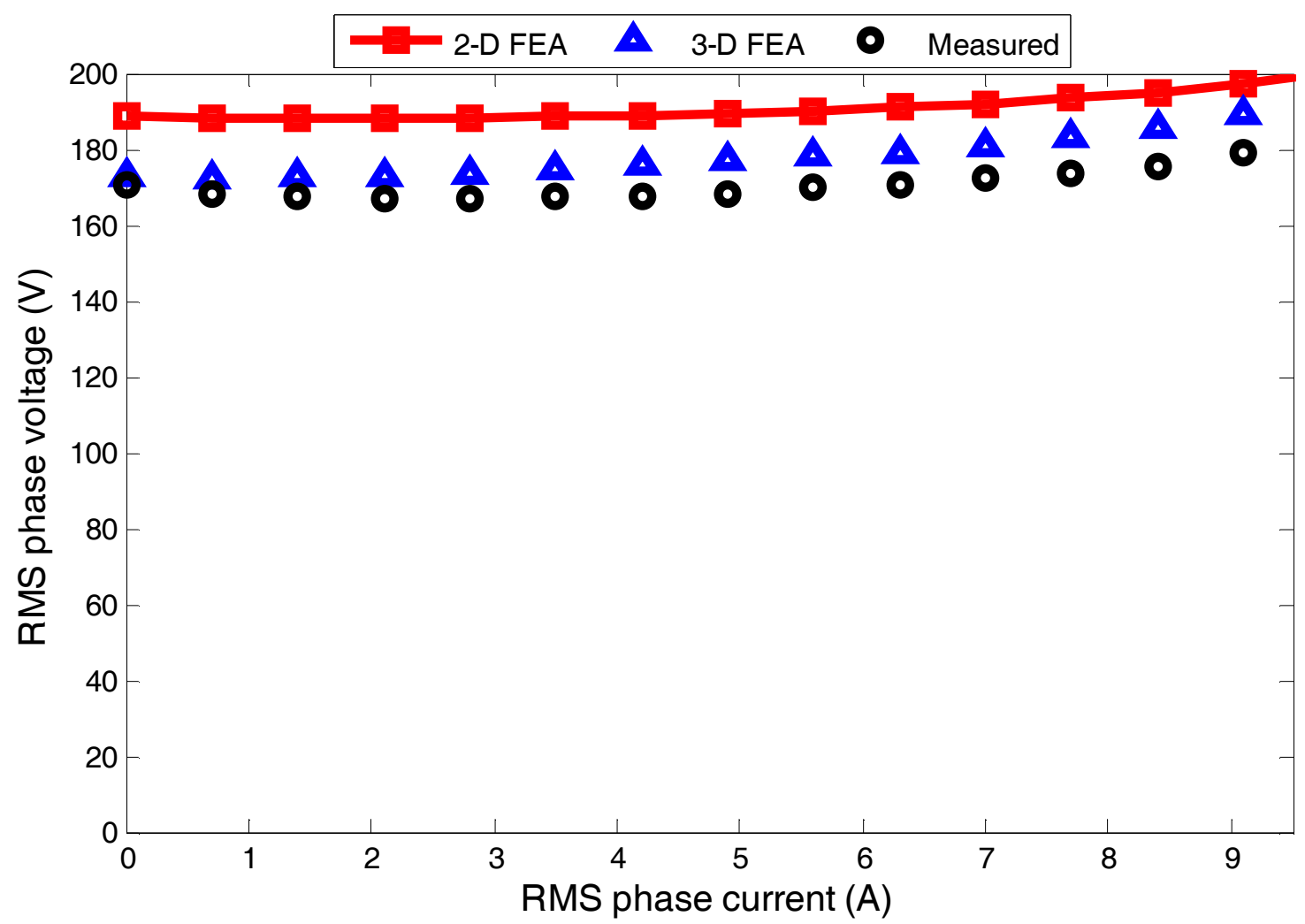

Fig. 7.23. Comparison of FEA and experimental results of the load current characteristics on the phase voltages for the WF-FSM prototype at $\sim 180 \mathrm{r} / \mathrm{min}$.

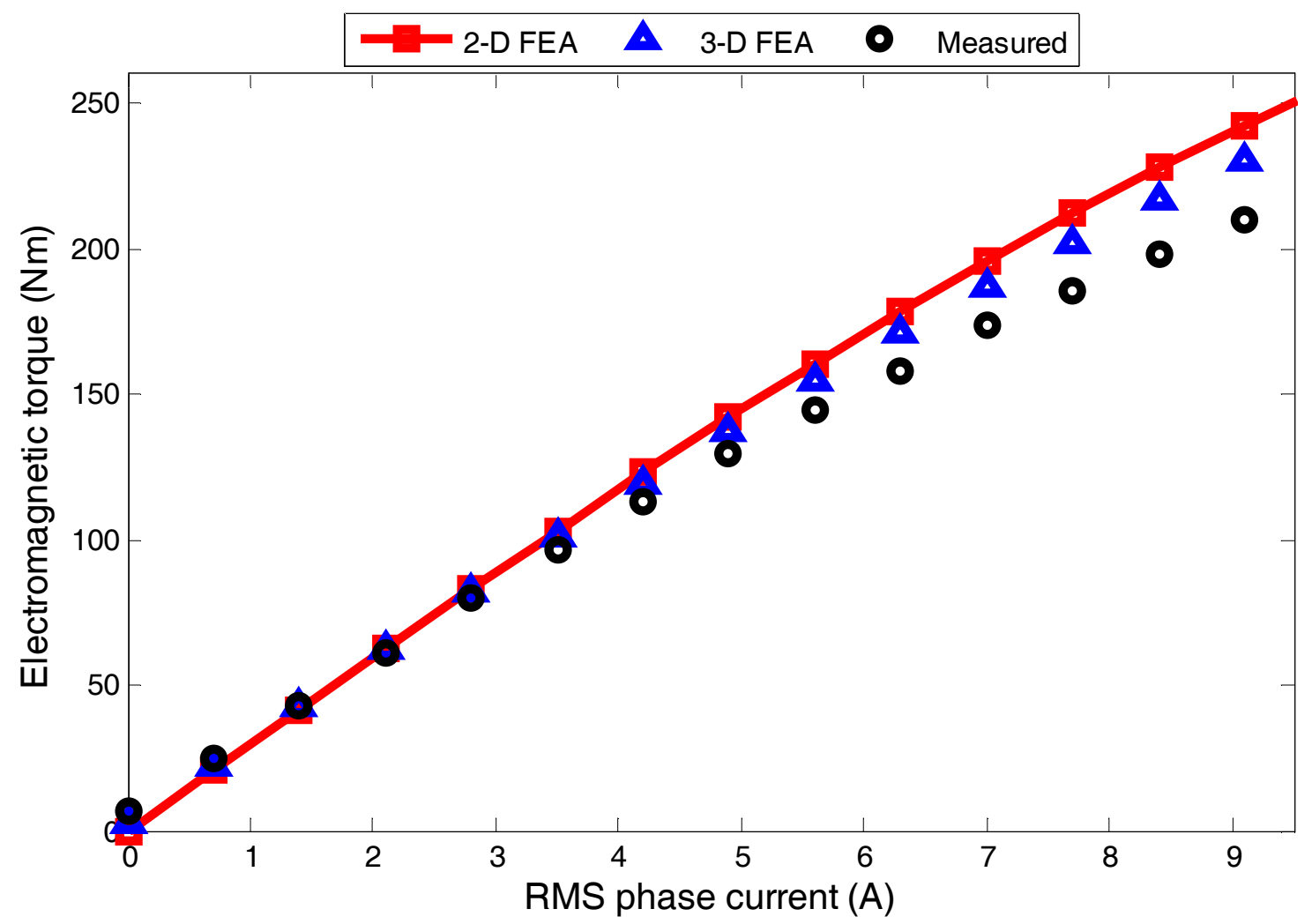

Fig. 7.24. Comparison of FEA and experimental results of the load current characteristics on the average torque evaluated for the WF-FSM prototype. 


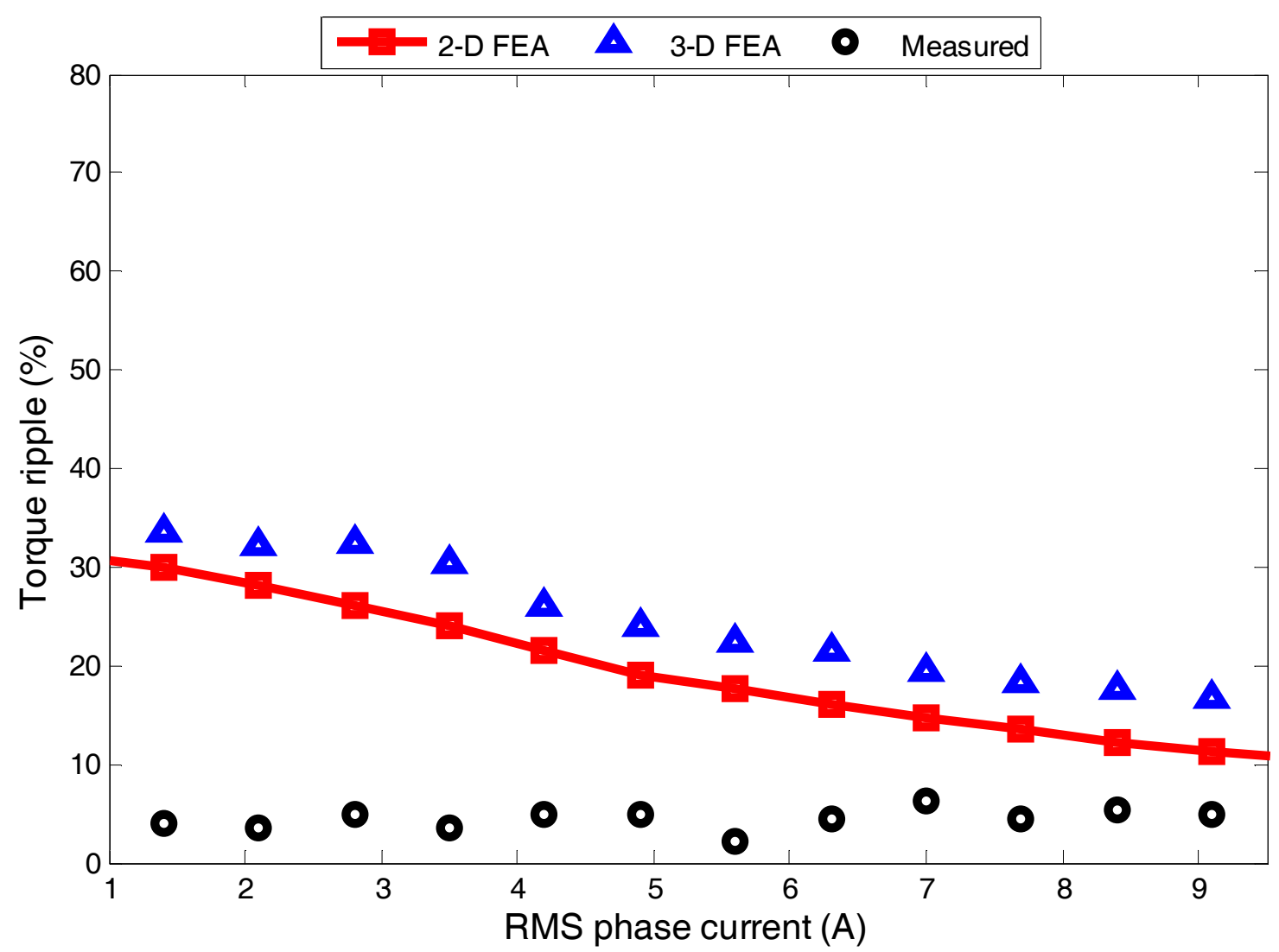

Fig. 7.25. Comparison of FEA and experimental results of the load current characteristics on torque ripple for the WF-FSM prototype.

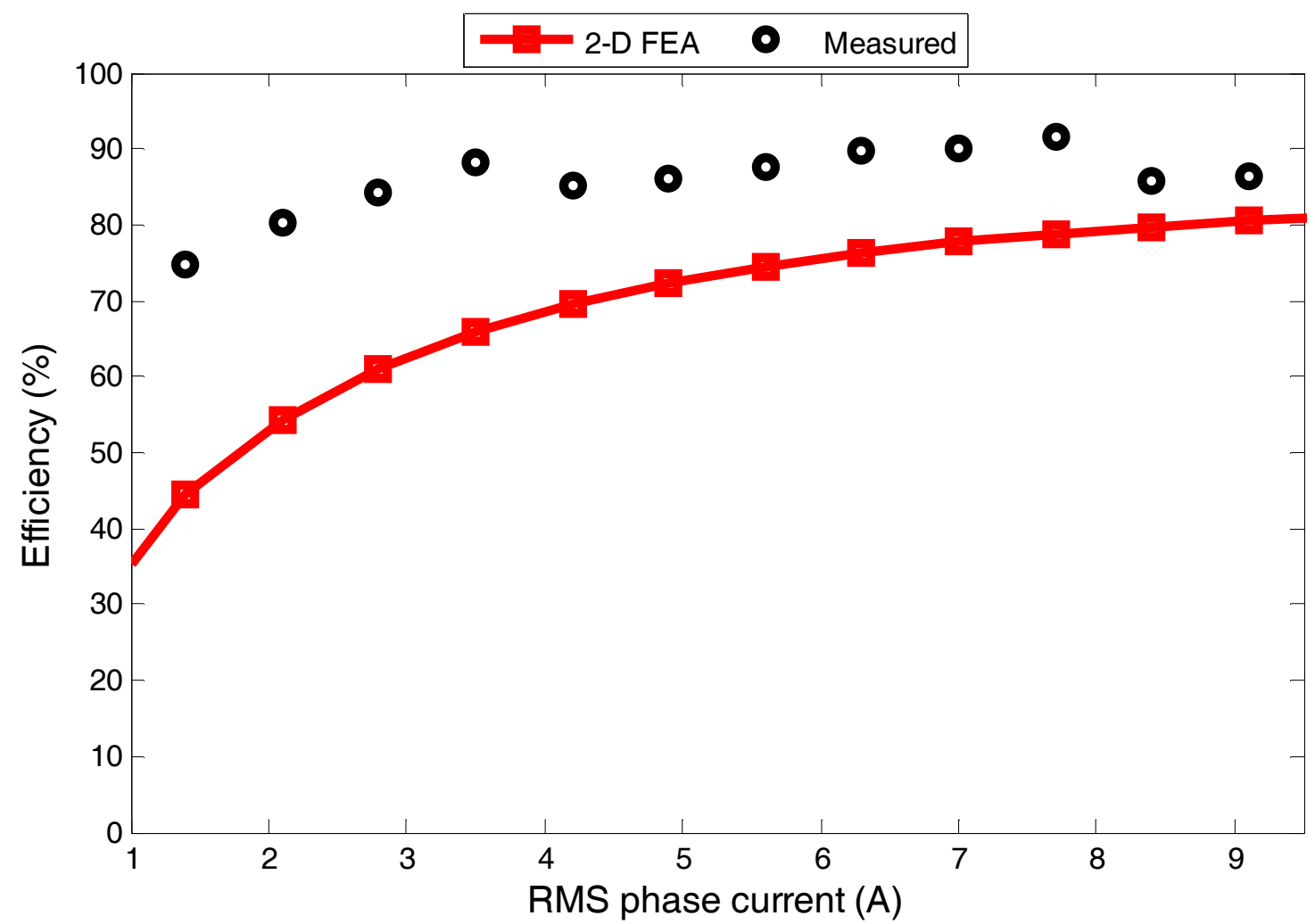

Fig. 7.26. Comparison of FEA and experimental results of the load current characteristics on efficiency for the WF-FSM prototype at $\sim 180 \mathrm{r} / \mathrm{min}$. 


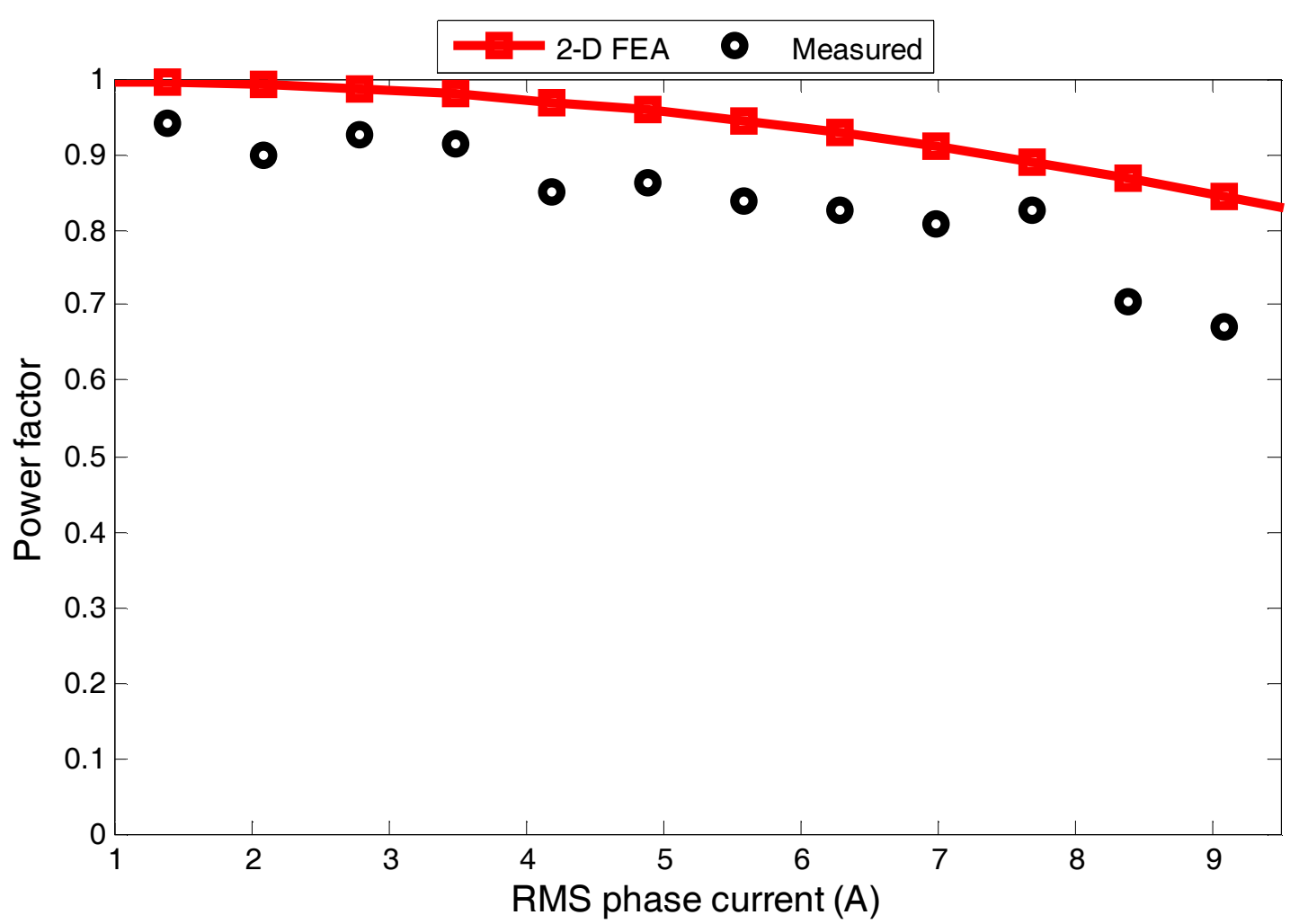

Fig. 7.27. Comparison of FEA and experimental results of the load current characteristics on power factor for the WF-FSM prototype at $\sim 180 \mathrm{r} / \mathrm{min}$.

Meanwhile, it is observed in Figs. 7.25 and 7.26 that the measured results for torque ripple and efficiency performed better than FEA calculations for the entire load range, respectively. While it is both interesting and surprising, it is notwithstanding very difficult to articulate the reason for improved torque ripple in the measured results, especially given that certain structural limitations were encountered during the process of fabrication and testing such as, an uneven airgap length, parallel winding connections and PWM switching, to mention a few. Unlike in FEA, the measured torque ripples are apparently able to maintain fairly constant values of less than $10 \%$ peak-to-peak.

However, it is not far-fetched to relate the improvement in efficiency to the significant differences earlier observed in the wound-field resistance, between FEA and measured results, in Table 7.4. The indicated field resistive drop for the measured value ( $-26.31 \%$ compared to $2-\mathrm{D})$, no doubt, is bound to yield a corresponding improvement in the efficiency of machine.

Generally, the measured results, based on the applied current-controlled tests, exhibit comfortable agreement with those predicted via FEA. Based on this outcome, the voltage regulation (VR) of the generator prototype can be admitted. As indicated in Simões, Farret and Blaabjerg (2015) [143], VR is an important figure of merit for the choice of wind generators, which expresses their ability to maintain a fairly constant supply voltage on the grid-side in spite of potential load transients. Besides, the variation of the wind energy resource and the proliferation of distributed wind generating units are precursors to voltage fluctuations in electrical networks. 
Table 7.5. Compared voltage regulation of test generator at $\sim 180 \mathrm{r} / \mathrm{min}$

\begin{tabular}{c|c|c}
\hline 2-D FEA & $3-$ D FEA & Measured \\
\hline \hline$-6.78 \%$ & $-7.67 \%$ & $-6.05 \%$ \\
\hline
\end{tabular}

Accordingly, Patel (1999) [3], indicated that the acceptable VR is typically 5-7 \% in developing countries. Hence, the VR of the manufactured prototype is determined and compared with FEA as shown in Table 7.5. The negative sign is an indication of potential rising characteristics of the supply voltage with respect to load variations. The estimated low values are also indications of very good voltage regulation. However, the highpoint of the fact is that, despite the observed voltage fluctuations, by opting for the WF-FSM wind generator topology, the designer is able to maintain a fairly constant supply voltage under time-varying wind speeds by simply regulating the DC of the wound-fields.

\subsection{Cost Implications}

Lastly, the quest to establish lighter and cheaper designs for the proposed wind generator drivetrain is not to be ignored in this chapter. For that reason, the researcher has provided a summary of the evinced masses and costs of the constructed prototype as shown in Fig. 7.28. This summary, which dwells on the individual stator and rotor assemblies, for the bulk of the estimates, reflect the material costs of the $10 \mathrm{~kW}$ WF-FSM prototype designed in this study. The costs of the main components and their accessories are also distributed as shown in Fig. 7.29. Interestingly, the rotor design robustness is once again proven by the fact that the rotor laminations presented the least material cost.

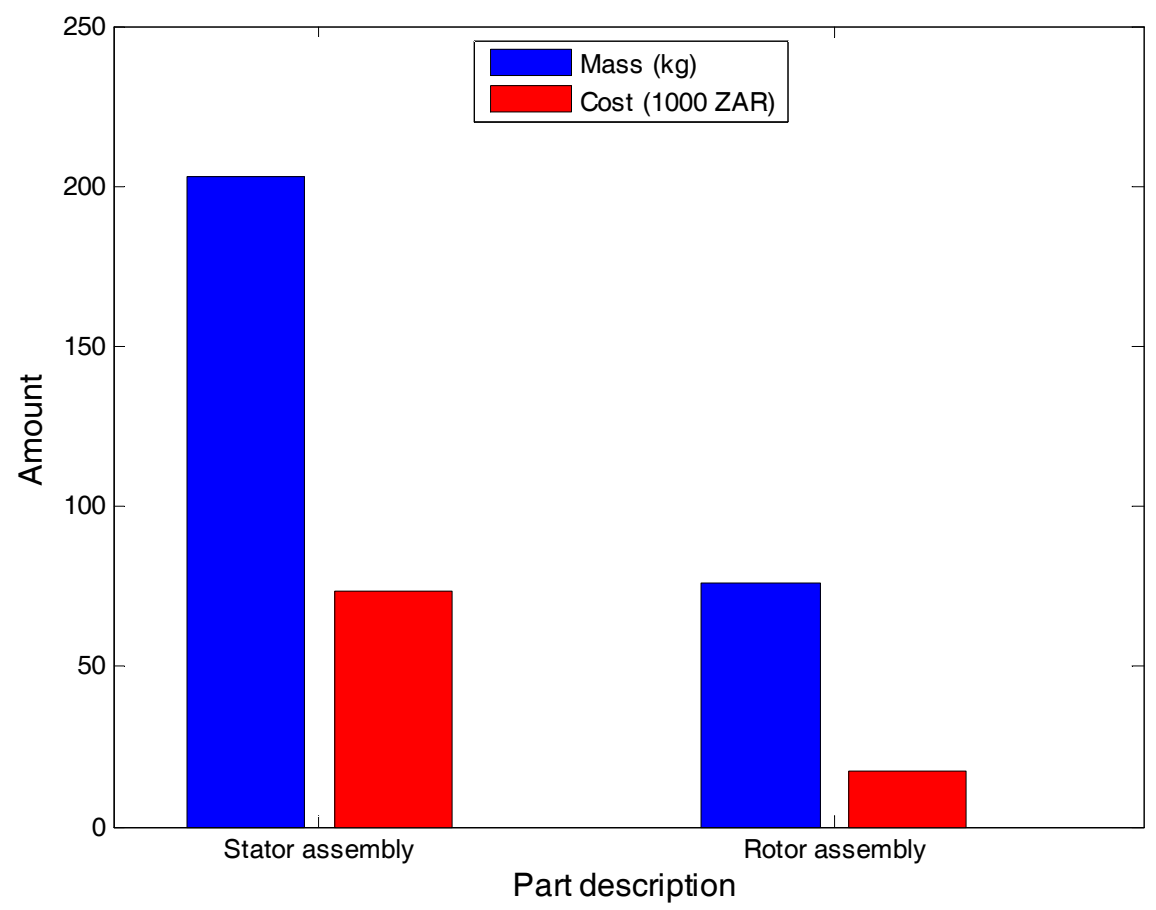

Fig. 7.28. Mass and cost of materials used in the manufacturing of the $10 \mathrm{~kW}-\mathrm{FSM}$ prototype. 


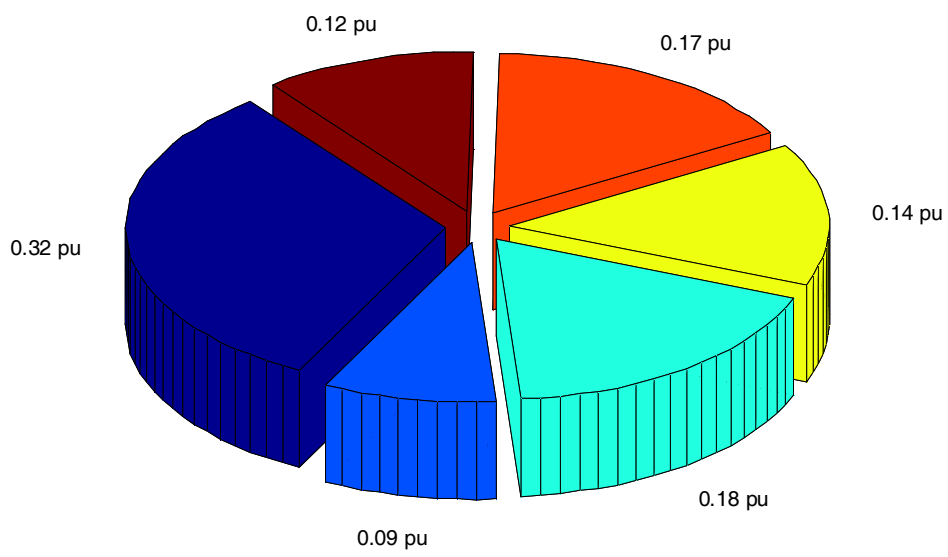

Fig. 7.29. Distribution of costs among the different parts of the manufactured prototype (base value $=$ total material cost of manufactured prototype).

\subsection{Chapter Summary}

In this chapter, the development and testing of a $10 \mathrm{~kW}$ prototype based on the proposed 12/10 radial-flux WF-FSM, designed for geared MS wind generator drives, has been presented for the first time. A simplified manufacturing approach has been adopted as well, which led to the use of not only prefabricated AC, but DC coils. The ease of logistics, installation and maintenance of the proposed modularised stator lamination design along with the winding preformation is also not to be overlooked, especially for generators designed for large-scale wind power applications. Based on measured no-load, short-circuit, thermal, uncontrolled-normal and overload resistance, as well as current-controlled tests, the design feasibility as well as the conceptualisation of the proposed wind generator drivetrain has been proven beyond reasonable doubt. Although, it has to be said that some infractions incurred on the prototype at the manufacturing stage, as well as unaccounted leakages and a complex magnetic coupling of the DC and AC windings in the FEA predictions, resulted in certain discrepancies in the obtained measurements.

Particularly, due to such underestimation of the high occurrence of end leakages upon saturation of the iron cores and not accounting for the complex magnetic coupling between the field and phase flux linkages in 2-D FEA, a reduction in the power density and power factor were observed in the on-load characterisation of the manufactured prototype, even at lower load current profiles. However, across the observed load current profile, the measured efficiency better than FEA results due to corresponding decrease in the measured field resistance. Despite certain the manufacturing and testing odds, fairly constant but lower torque ripple values were also encountered on the manufactured prototype, compared to FEA predictions, from no-load to rated conditions. This observation is 
very intriguing and not easy to elucidate. Also, the higher efficiency, which although is readily attributed to decrease in the measured field resistance, is interesting for the machine prototype because it takes into account the mechanical losses hitherto ignored in the FEA design stage.

In the course of the short-circuit tests, heat runs were conducted whereby it was observed in different hotspots of the field and phase coils that the steady-state temperature can be attained just after the hour mark, and that the field coils heat up twice as much than the phase coils, and faster, especially at the knee points. It is further established that the machine can be safely overloaded without stressing its thermal limits. Consequently, in spite of several bottlenecks encountered during experimentation, the correlation of the measured results of the fabricated prototype to the design approach propounded in this dissertation is proven. 


\section{Chapter Eight}

\section{Concluding Remarks}

\subsection{Conclusion}

In this dissertation, the multi-objective design optimisation of radial-flux FSMs processed via 2-D static FEA have been undertaken to evaluate the performance of their optimal design topologies for geared MS wind generator drives, especially in terms of potentials for rare-earth-free designs at industrial-scale power levels. Some of the major conclusions reached during the study are summarised as follows:

I. Rare-earth PM-FSM drivetrain performance comparison for $10 \mathrm{~kW}$ machines: It has been found that by comparing the three different drivetrain topologies (i.e., LS, MS and HS), based on the optimal performance of the $12 / 10$ generators at $10 \mathrm{~kW}$ power, the HS drivetrain, which employs maximum gearbox size and cost, yields the cheapest design but at the expense of utilising the most amount of PM per average torque produced (at not more than $35 \mathrm{Nm} / \mathrm{kg}$ ), as well as producing the highest core losses. On the other hand, the LS drivetrain is found to use the least amount of PM compared to its average torque range $(47-67 \mathrm{Nm} / \mathrm{kg}$ ), but it results in oversized machines with proportional increase in the cost of the active materials used, beyond other apparent potential logistical costs. Moreover, the implication of the oversized LS designs, when compared with the rest, shows that it displays the worst efficiency and best power factor. The excellent power factor in the LS design can be attributed to the uniform airgap assumed for all three drivetrains; thus with highest power factor, it is assumed that the inverter costs would be minimal. Generally, all three $10 \mathrm{~kW}$ PM-FSM drivetrains exhibit positive correlation between power factor and efficiency as a function of their resolved current densities, which means that power factor improves as efficiency increases. Although, the evolved optimal current densities of the LS design is approximately $50 \%$ compared to values observed in the HS and MS designs, it is still considered as very high to limit the $I^{2} R$ losses. Hence, to further decrease the current density of the LS design in order to improve its efficiency, it was found that a proportional decrease is required for the optimal split ratio. A possibility therefore arises for the LS machine to achieve unitary power factor at less than $95 \%$ efficiency, but at the risk of increasing its PM utilisation. Interestingly, the MS drivetrain yields designs that produce tradeoffs in terms of torque-PM densities as high as the LS design $(37-57 \mathrm{Nm} / \mathrm{kg}$ ) and at generator costs comparable to those of the HS design, while fully satis- 
fying the torque ripple, efficiency and power factor requirements. Thus, the MS design is considered the preferred solution because it diminishes the extreme cost implications inherent in the LS generator and the HS gearbox, while maintaining an acceptable performance index.

II. Evaluation of rare-earth PM-FSMs at $10 \mathrm{~kW}$ and $3 \mathrm{MW}$ power levels: It is shown that the 12/10 and 12/14 machines when optimised for multi-objective problem formulations, the estimated torque ripple in the $3 \mathrm{MW}$ designs are much lower compared to the $10 \mathrm{~kW}$ designs. Similarly, from the results obtained, it is also shown that the torque densities and torque ripples are significantly improved at $3 \mathrm{MW}$. The torque ripples perform better at $3 \mathrm{MW}$ because their evolved machines display lower saliency ratios compared to the $10 \mathrm{~kW}$ machines. However, the performance of the 12/10 and 12/14 machines when compared at the same power level, vary differently. While the 12/14 machine is able to record smaller active mass and torque ripple compared to the $12 / 10$ machine at $10 \mathrm{~kW}$, the reverse is the case at $3 \mathrm{MW}$. It was clearly shown that the optimal resolution of certain design variables in the MDO process, such as the PM volume, aspect ratio, split ratio and current density, is responsible for the observed performance alterations. For instance, the higher the PM utilisation factor, the higher the torque ripples. Also, it was discovered that for the utilised MDO strategy, the theoretical performance of the 12/14 machine, which prescribes higher core loss and higher torque capability due to relatively higher number of rotor poles compared to the $12 / 10$, is discredited when imposed in a MDO environment. Generally the $3 \mathrm{MW}$ machines displayed superior performance which provides insights on their high potentials for utility-scale wind turbine systems, as well as a possibility for employing rare-earth-free materials.

III. Performance comparison of $10 \mathrm{~kW}$ rare-earth-free designs: The possibility of using rareearth-free materials e.g., ferrite PMs and wound-fields, in the design of the 12/10 machine, as proposed for MS wind generator drives, has been established. The outcome from a series of MDO procedures show that-

a. the active mass of ferrite PM-FSMs are significantly minimised compared to the WFFSMs, whereby increasing the mass of the former, decreases the torque ripple, with two times the same tendency observed in the latter. Such discrepancies in optimal performance are associated to larger optimal aspect ratios for the ferrite PM-FSMs typical of PM machines, as well as larger split ratios for the WF-FSMs with field coils with higher propensity for copper losses.

b. based on certain benchmark designs, better efficiency ( $4.2 \%$ higher), torque ripple (55.2\% lower) and torque density (181.25\% higher) are observed in the ferrite PMFSM at only $50 \%$ of the total material cost compared to the WF-FSM design. 
c. when compared at par to a rare-earth PM-FSM benchmark, the cogging torque, torque ripple and torque density in a benchmarked ferrite PM-FSM, performs second best. However, a slight advantage is displayed in the machine efficiency along with a major cost savings of $35 \%$ in the ferrite PM-FSM design. Moreover, the occurrence of deeper saturation which limits the demagnetisation withstand ability is observed within normal operating conditions in the rare-earth design compared to the ferrite PM-FSM. This finding is central to the proposed MS wind generator applications, because ferrites are debuted for higher demagnetisation risks given their low-energy profiles.

IV. Formulation and MDO of only WF-FSMs at $10 \mathrm{~kW}$ power: The basic dq formulations and MDO strategy of WF-FSMs for wind generator drives has been reported, whereby the perspective and influence of different MDO approaches for WF-FSMs has been uncovered. Specifically, the results show that while concentrating the MDO on minimising the cost of the generator, a limitation on the performance is observed which in turn increases the total drivetrain costs much more than were the MDO tackled on improving the performance of the generator. In the pursuit of increased performance, an apparent tradeoff portrayed in Pareto data was uncovered between power factor and torque ripple of WF-FSMs. Thus, it implies that selecting a machine with minimum torque ripple, results in inferior power factor which is bound to increase the size and costs of the power inverters, and vice versa.

V. Design characterisation on the power-level scalability of rare-earth-free designs: The study which involved the comparison of both the $12 / 10$ and $12 / 14$, as well as their powerlevel scalability from $10 \mathrm{~kW}$ to $3 \mathrm{MW}$, and as usual processed using 2-D static FEA in conjunction with the MDO strategy, showed that:

a. like for rare-earth PM-FSMs, the performance of the $3 \mathrm{MW}$ ferrite PM-FSMs and WF-FSMs were much more improved, in terms of minimum active mass and torque ripple values, compared to the $10 \mathrm{~kW}$ designs.

b. the $12 / 10 \mathrm{WF}-\mathrm{FSMs}$ surprisingly produce significantly lower torque ripples than expected, when compared to the 12/14 WF-FSMs, at equal power levels. This is a further indication that with the MDO strategy, whereby certain FSMs are operated within a prevailing feasible environment and based on a cocktail of multiple conflicting design and performance quantities of which the designer has limited influence, results in outcomes which differ from typical situations wherein the design space is restricted.

c. the presence of wound-field coils and the theoretical limitation on the core losses of the $12 / 14$ machines were discovered to have deteriorated the efficiency requirements 
of the WF-FSM variants at both investigated power levels.

d. the 3 MW WF-FSMs resulted in smaller active mass compared to the $3 \mathrm{MW}$ ferrite PM-FSMs due to compromises between the optimal armature current densities and aspect ratios. Note that, the operating temperature of the copper-intensive WF-FSMs was prescribed significantly higher compared to the ferrite PM-FSMs, which further elevates the observed optimum characteristics of the 3 MW WF-FSM despite its slightly lower efficiency.

e. the torque ripple values recorded in all the considered power levels and for the different rare-earth-free machine topologies showed no major improvements when compared to rare-earth PM designs. Thus, the widely held assumption that the use of rareearth-free materials is most likely to douse the high torque ripple profiles in FSMs, implicated by its flux focusing abilities, appears to be a misconception.

f. by comparing the proposed $3 \mathrm{MW}$ rare-earth-free optimum design candidates with a similarly rated MS-designed DFIG and PMSG systems reported in the literature, the WF-FSMs represented the cheapest options in terms of total material costs, although the former systems are still capable of higher torque densities.

g. generally, ferrite PM-FSMs perform better at small-scale power levels, while WFFSM are better at large-scale power levels, for the proposed geared MS wind generator drivetrains.

VI. Construction and testing of a $10 \mathrm{~kW}$ prototype: The development and prototyping of a 10 kW WF-FSM benchmarked from the study undertaken in Chapter 5 has been presented, without any previous reference on such design ventures. The design feasibility and structural simplicity is facilitated by the implementation of the popular segmentation of the stator laminations as commonly utilised in similar PM-FSM topologies, therefore enabling the preformation of not only the phase coils, but the wound-field coils. The extended benefits of such design patterns may include improved means of construction and transportation, as well as reduced installation and maintenance costs, especially for large-scale power applications. Hence, the structural proofs of the manufactured prototype have been fully detailed beginning from the electromagnetic design to mechanical drawings, artistic impressions to actual fabrication of the component parts, and assembly to final tests in the laboratory. Basic no-load, short-circuit, thermal, uncontrolled-normal and overload resistance, as well as currentcontrolled experiments undertaken on the manufactured prototype have proven the FEAbased MDO technique generally adopted in this dissertation to be correct within some slight margin of errors. The observed discrepancies between measured and predicted results were 
mainly caused by infractions on the original design template during the manufacturing process, sidelining certain existent magnetic coupling between the DC and AC windings, as well as underestimating the end leakages at the FEA design stage. In particular, slight reductions in the power density and power factor were observed when the prototype machine was tested on-load. However, the efficiency and torque ripple profile performed better than those predicted in FEA. The improved efficiency is due to decrease in the measured field resistance compared to FEA, while that of the torque ripple is not easy to justify. The heat runs conducted during the short-circuit tests; show a steady build-up of temperature within just one hour, with the main temperature hotspot identified in the field coils. Also, the thermal runs proved that the WF-FSM can be overloaded without harmful performance limitations. In conclusion, there was general correlation between the predicted and measured results which establishes the prospect of the proposed wind generator drivetrains for further developments for industrial approval.

\subsection{Aspects on Novelty}

Beyond the conclusions already reached, the important aspects on novelty as proposed and reported in this dissertation is as follows:

I. The MDO strategy as widely adopted in this dissertation has never been used to evaluate the performance of the proposed 12/10 and 12/14 radial-flux FSM for geared MS wind generator topologies. Moreover, with the assistance of the sizing-power technique, and an in-house 2 D static FEA program, a robust design methodology is harnessed. Note that, with such methodology, it is expected that the procedure be administered as a multi-objective, multiconstrained, and multi-variable problem as emphasised by Duan and Ionel (2013) [87]. In the majority of cases, parametric resolutions such as utilised in Ojeda et al (2012) [37] and Chen et al (2009) [82], as well as non-gradient processes as done in Somesan and Viorel (2013) [68], have prevailed. To this end, the important finding observed in the use of MDO in the current study is that some theoretical performance expected of certain FSM topologies may be discredited because of the high-level competition that is promoted within the feasible design space. For example, it has been serially established in this thesis that the 12/14 machine for certain topologies and power levels may not always display superior torque and torque ripple, nor will it always yield poor efficiency, compared to its $12 / 10$ counterpart as claimed in Chen et al (2009) [82]. Another example is that when different MDO approaches of the $12 / 10 \mathrm{WF}-\mathrm{FSMs}$ designed at $10 \mathrm{~kW}$ power levels are considered, an interesting outcome is observed such that a compromise which exists between the power factor and the torque ripple 
values is uncovered for the first time. The case of power factor versus torque ripple presents a situation wherein the performance of the wind generator is prioritised, with influential drivetrain cost reductions occurring at a negotiable increase of the generator costs. However, when the manufacturing costs are so prioritized, by minimising the components' mass, then the generator cost is reduced but at the expense of dramatically increasing the overall drivetrain costs. In essence, by undertaking multiple MDO processes for different topologies and configurations of the proposed machines, the researcher has been able to show beyond doubt that the use of MDO strategies, viz., non-gradient optimisation processes, gives not just a global perspective of the nonlinear multimodal search space defined in FSMs, but also guarantees a myriad of design options which becomes readily available for the designer to make informed selections. Therefore, the unique information provided in the Pareto optimal sets adjudicated in this thesis, may be lost in the presentation of single-objective problems and/or by the use of gradient-based methods. That the main limitation of MDO processes is the computational costs required for such efforts, especially when coupled to FEA, is not in doubt to the researcher, but nevertheless, it is beyond the scope of the present research.

II. In the published literature, the sizing and design of PM-FSMs is not lacking as seen in Zhang et al (2009) [45], Hua et al (2006) [61] and Somesan and Viorel (2013) [68]. But in terms of WF-FSMs, the researcher happens to be the first person to emphasise the same technique in order to facilitate their MDO strategies. Also, the researcher is able to lay out simple dq formulations on the analyses of WF-FSMs, which was further escalated by streamlining their end-winding calculations based on the formula proposed for non-overlap PM winding machines in Potgieter and Kamper (2014) [94]. The devised end-winding calculation is considered approximate for the MDO processes pursued in this dissertation, unlike the technique in Zhou and Zhu (2014) [70] which considered the resistance but ignored the inductance or that of Raminosoa et al (2015) [71] who only prescribed a fixed margin to account for such effects. Furthermore, such consideration is necessary especially because of the arrangement of the phase coils over the wound-fields of the proposed WF-FSM topology, coupled with the fact that a good number of large-scale power designs have been considered in this thesis.

III. The proposal made in this study on the designed machines for geared MS wind generator drive is unique to the researcher. The motivation was fueled by the potentials of such drivetrain technology in terms of best performance to cost index for other wind generator concepts, DFIG_-Cao, Xie and Tan: 2012 [4], PMSG—Schmidt and Vath: 2012 [20] and PMSG-Bang et al: 2008. Now, the researcher has added PM-FSMs, viz., FSMs to the list, whereby similar outcomes of the MS drivetrain compared to LS and HS systems have been 
obtained at $10 \mathrm{~kW}$ power levels. For geared MS drives, no doubt the potentials of the 12/10 and 12/14 topologies have also been established, first for rare-earth PM designs, and then for rare-earth-free designs. By realising large-scale designs, which are better performing, is to be credited with the fact that these machines would be profitable to the WPG industry. The common stigmas associated with FSMs such as high torque ripple values have been shown to become diminished in the large-scale power designs. Additionally, as per the quest to transit from rare-earth designs to rare-earth-free designs, the emphasis for wind generator applications of the proposed FSM topologies is novel to the researcher, unlike for other applications where such emphasis has been mostly in terms of automotive drives e.g., Fasolo, Alberti and Bianchi (2014) [69] and Raminosoa et al (2015) [71]. Also, the researcher witnessed a trend, whereby the torque ripple values do not fare any better for the rare-earth-free compared to rare-earth designs after the MDO process. This observation is contrary to insinuations expounded in Hua and Cheng (2008) [139], Wang, Wang and Jung (2012) [140], Shen and Fei (2013) [141] and Sikder, Husain and Ouyang (2015) [48], wherein it is insinuated that the high cogging torque cum torque ripple in FSMs, partially caused due to flux focusing effects, may be suppressed using rare-earth-free materials. Furthermore, it was discovered that for small-scale power designs, ferrite PM-FSMs are preferable to WF-FSMs, while for largescale designs, the potential benefits lie in the latter design topologies. To this end, the consideration of rare-earth-free designs for the proposed wind generator topologies have never been attempted at large-scale power levels e.g., at $3 \mathrm{MW}$, as undertaken in this dissertation. A scarce argument can be made for studies done by Wang et al (2013) [81] in their high temperature superconductor WF-FSM design and Ditmanson et al (2013) [84] in their novel $500 \mathrm{~kW}$ modular PM-FSM drive topology. However, their implementations mainly focused on the LS drivetrain, among other things. Apparently, the large-scale power investigation considered in this dissertation is a new initiative for the 12/10 and 12/14 machine drive topologies.

IV. Lastly, the development and testing of a $10 \mathrm{~kW}$ WF-FSM prototype of the proposed 12/10 topology has never been reported. A look in the published literature reveals that it is only Tang et al (2012) [67] who really had anything to do with these machine topologies. Hence they only studied the 12/14 topology based on FEA techniques for automotive drive applications. Without doubt, the researcher understands that the original appearance as proposed by Tang et al (2012) [67] might have posed a lot of manufacturing concerns to other researchers, including Tang et al, in the later years. For instance, imagine if the DC coils were to be wound manually for large-scale power designs: of what design feasibility is this? Thus, the researcher is confident that he is the first person to actually lay down a design template (puz- 
zle-like segmentation of the stator laminations) for the prototype development and manufacturing of these machine topologies, such that the process is not only simplified, but has now opened up a vista of opportunities which would make them attractive to upcoming researchers and the industry. Whereby the researcher cannot claim that the manufacturing of the $10 \mathrm{~kW}$ WF-FSM prototype was an entirely stress-free process, he is nevertheless certain that the structural modifications made on the design proof did, and does, immensely facilitate its manufacturing.

\subsection{Recommendations}

Based on the findings reported in this dissertation, the following recommendations, which are not limited, may apply to future research and development:

I. The drivetrain comparison done in Chapter 2 only considered the proposed wind generator for the 12/10 rare-earth PM topologies at $10 \mathrm{~kW}$ output power, with other drivetrain components such as the gearbox and SSCs considered as mere estimates. Thus, it would be interesting to optimally design and compare the wind generator performance quantities of the $12 / 10,12 / 14$ or other simple radial flux FSM topology in tandem with their associated drivetrain components e.g., none-rare-earth designs for large-scale power levels.

II. The MDO reported in this dissertation is strictly based on genetic algorithm, which is very accurate but time exhaustive for such design problems. It would be comforting if future research works could consider the use of other robust non-gradient optimisation techniques, for the sake of improving on the simulation speed, e.g., so-called differential evolution as used in Duan and Ionel: 2013 [87] for PMSM and Zhang, Ionel and Demerdash (2016) [89] for IPM machines.

III. No doubt, as observed in most of the 3-D transient FEA solutions and the experimental test reported in this dissertation, the saturation effects in FSMs is generally high, even higher for the proposed WF-FSM topologies especially when operated close to rated conditions. Due to the flux linkages in the field windings varying with time, voltages become induced in the would-fields, with very telling consequence on the machine characteristics. One way this can be corrected, as shown in Mabhula and Kamper (2017) [138] for WRSM designs and GuangJin, Zhu and Jewell (2015) [118] for HE-FSMs, is by employing the frozen permeability (FP) technique to facilitate the steady-state 2-D static FEA calculations. It is anticipated that the FP technique could help to accurately portray the cross-magnetisation and saturation effects on the rated machine performance characteristics. Moreover, by adopting the FP approach, it is expected that such limitations encountered by the use of simple dq expressions in evaluat- 
ing the torque ripple values as reported in Chapter 5, may be resolved. Another way is to adopt the technique proposed in Zulu, Mecrow and Armstrong (2010) [147] for segmentedrotor WF-FSM to correct the discrepancies observed between predicted and measured results of $10 \mathrm{~kW}$ WF-FSM presented in Chapter 7, and also to consider Sikder, Iqbal and Ouyang's (2016) [142] work. Their approaches involve using coupling coefficients to improve the correlation between predicted and measured results.

IV. Given the proposal established on radial-flux FSMs for MS wind generator designs in this thesis, there are yet so many other established and emerging concepts and topologies differing in terms of stator-slots/rotor-pole number combination, active flux direction, phase number configuration, excitation system, drivetrain topology, etc., that have not been attempted for wind generator applications. These areas are available to be further investigated and even compared with the topologies already presented in this thesis.

V. The use of HTS windings in the design of a different topology of the radial-flux WF-FSMs as conveyed in Wang et al (2013) [81] is also interesting to be considered in the WF-FSM topology proposed in this dissertation, granted that a significant decrement in the size of the wound-field coils in response to the MDO process has already been reported for designs in the MW power range in Chapter 6. Perhaps, such HTS WF-FSM design might provide just the necessary impetus to push their performance to reach comparable torque densities with other industrial-scaled conventional geared MS wind generators, while having already attained a status as the cheapest design in that power range.

VI. With basic current-controlled experimentation implemented on the $10 \mathrm{~kW}$ WF-FSM prototype developed in this thesis, the proposed MS wind generator concept has been established beyond doubt. But yet to be fully initiated are actual field tests; such ventures are not yet reported and are thus very much open to the future research interests. Besides, as undertaken in Potgieter (2014) [85] for SS-PMGs, it also remains to be seen how the performance of the proposed FSMs, especially the WF-FSM design, would fare in a directly grid-connected wind system, whereby the need for inverters are totally diminished. 


\section{REFERENCES}

[1] REN 21, Renewables 2016 Global Status Report (Paris: REN21 Secretariat), 2016. [Online]. Available: www.ren21.net/gsr

[2] DNV/Risø - Det Norske Veritas and Risø National Laboratory, Guidelines for design of wind turbines, 2nd ed., Jydsk Centraltrykkeri, Copenhagen, Denmark, 2002.

[3] M. R. Patel, Wind and solar power systems, CRC Press LLC: Boca Raton: Florida, 1999.

[4] W. Cao, Y. Xie and Z. Tan, Wind turbine generator technologies, InTech, 2012. [Online]. Available: https://www.intechopen.com/books/advances-in-wind-power/wind-turbine-generator-technologies

[5] J. F. Manwell, J. G. McGowan and A. L. Rogers, Wind energy explained, John Wiley \& Sons Ltd: West Sussex, England, 2002.

[6] REN 21, Renewables 2016 Global Status Report (Paris: REN21 Secretariat), 2017. [Online]. Available: www.ren21.net/gsr

[7] H. Polinder, J. A. Ferreira, B. B. Jensen, A. B. Abrahamsen, K. Atallah and R. A. McMahon, "Trends in wind turbine generator systems," IEEE Journal of Emerging and Selected Topics in Power Electronics, 1(3), pp. 174185, Sept. 2013.

[8] IRENA Working Paper, Renewable energy technologies: cost analysis series, Wind Power, Volume 1: Power Sector, Issue 5/5, June 2012. [Online]. Available: https://hub.globalccsinstitute.com/sites/default/files/publications/138178/hydropower.pdf

[9] T. Vandendael, "Cost analysis of a wind turbine", GROEP T-MY STATEMENT BLOG, Feb 18, 2013. [Online]. Available: https://gtms1303.wordpress.com/2013/02/18/cost-analysis-of-a-wind-turbine/

[10] Z. Q. Zhu and Jiabing Hu "Electrical machines and power-electronic systems for high-power wind energy generation applications: Part I--market penetration, current technology and advanced machine systems," The international journal for computation and mathematics in electrical and electronic engineering (COMPEL), 32(1), pp.7-33, 2012.

[11] H. Polinder, F. F. A. van der Pijl, G. J. de Vilder and P. J. Tavner, "Comparison of direct-drive and geared generator concepts for wind turbines," IEEE Transactions on Energy Conversion, 21(3), pp. 725-733, Sept. 2006.

[12] E. Aydin, "Determination of best drive train technology for future onshore wind turbines as a function of the output power", MSc Thesis, Department of Electrical Engineering, Eindhoven University of Technology, 2013.

[13] E. de Vries, ABB shifts focus to medium speed drive systems, WINDPOWER Monthly, 4 September 2012. [Online]. Available: http://www.windpowermonthly.com

[14] H. Gitano-Briggs, Low speed wind turbine design, InTech, 2012. [Online]. Available: https://www.intechopen.com/books/advances-in-wind-power/low-speed-wind-turbine-design

[15] H. Li, Z. Chen, and H. Polinder, Research report on numerical evaluation of various variable speed wind generator systems, Project UpWind, Technical Report, 2006. [Online]. Available: https://slideblast.com/researchreport-on-numerical-evaluation-of-various-_595fd9c11723ddda477d01 cd.html

[16] A. M. Ragheb and M. Ragheb, wind turbine gearbox technologies - fundamental and advanced topics in wind power, Dr. Rupp Carriveau (ed.), InTech, 2011. [Online]. Available: http://www.intechopen.com/books/fundamental-and-advanced-topics-in-wind-power/wind-turbinegearboxtechnologies

[17] ReliaWind Project, Reliability-focused research on optimising Wind Energy system design, operation and maintenance: tools, proof of concepts, guidelines \& methodologies for a new generation, Gamesa Innovation and Technology, Mar. 2008 - Mar. 2011. [Online]. Available: http://cordis.europa.eu/project/rcn/88411_en.html

[18] U. B. Akuru and M. J. Kamper, "Contemporary wind generators," Eleventh industrial and Commercial Use of Energy International Conference, ICUE, Cape Town, pp. 1-8, 2014.

[19] E. de Vries, The evolution of wind turbine drive systems, WINDPOWER Monthly, 26 April 2012. [Online]. Available: http://www.windpowermonthly.com

[20] S. Schmidt and A. Vath, "Comparison of existing medium-speed drive train concepts with a differential gearbox approach," European Wind Energy Association, EWEA, Copenhagen, pp. 179-186, April 2012.

[21] A. Matveev, "Novel PM generators for large wind turbines," Wind Power R\&D Seminar-Deep Sea Offshore Wind Power, Trondheim, Norway, 20-21 January 2011.

[22] P. Tavner, E. Abdi, M. Tatlow and R. McMahon "Design and performance analysis of a 5 MW medium-speed brushless DFIG drivetrain" EWEA, Austria, 2013.

[23] J. Coultate, Wind turbine drivetrain technology and cost drivers, Romax Technology, 2011. [Online]. Available: www.romaxtech.com

[24] A. Vath, Wind turbine drive train innovations and load reduction solutions, Bosch Rexroth AG, Sept. 2012.

[25] M. Ragheb, Modern wind generators, NetFiles, University of Illinois at Urbana-Champaign, 2/28/2014.

[26] N. Madani, "Design of a permanent magnet synchronous generator for a vertical axis wind turbine," MSc thesis, School of Electrical Engineering, Royal Institute of Technology, Stockholm, Sweden, 2011. 
[27] Y. Zou, Induction generator in wind power systems, InTech, 2015. [Online]. Available: https://www.intechopen.com/books/induction-motors-applications-control-and-fault-diagnostics/inductiongenerator-in-wind-power-systems

[28] M. R. Dubois, "Optimized permanent magnet generator topologies for direct-drive wind turbines," PhD Dissertation, Delft University Technology, 2004, 9-27.

[29] D-J Bang, H.P. Under, G. Shrestha and J. Ferreira, "Promising direct-drive generator system for large wind turbines," Wind Power to the Grid Seminar, EPE-WECS, 2008.

[30] R. S. Semken, M. Polikarpova, P. Roytta, J. Alexandrova, J. Pyrhönen, J. Nerg, A. Mikkola and J. Backman, "Direct-drive permanent magnet generators for high-power wind turbines: benefits and limiting factors," IET Renewable Power Generation, 6(1), pp. 1-8, January 2012.

[31] H. Chen, R. Qu, J. Li and D. Li, "Demagnetization performance of a $7 \mathrm{MW}$ interior permanent magnet wind generator with fractional-slot concentrated windings," IEEE Transactions on Magnetics, 51(11), pp. 1-4, Nov. 2015.

[32] S. Sjökvist, "Demagnetisation studies on permanent magnets—comparing FEM simulations with experiments", Licentiate Thesis, Uppsala Universitet, June 2014.

[33] C. Mademlis and I. Kioskeridis, "Optimizing performance in current-controlled switched reluctance generators," IEEE Transactions on Energy Conversion, 20(3), pp. 556-565, Sept. 2005.

[34] M. Ahmad, High performance AC drives: modelling, analysis and control (switched reluctance motor drives), Springer Heidelberg, Berlin, 129-160, 2010.

[35] I. Husain, A. Radun and J. Nairus, "Fault analysis and excitation requirements for switched reluctance generators," IEEE Transactions on Energy Conversion, 17(1), pp. 67-72, Mar. 2002.

[36] R. Karthikeyan, K. Vijayakumar, R. Arumugam, and V. Kamaraj, "Design and analysis of a switched reluctance generator for rural electrification in standalone wind energy conversion system," International Conference on Power Systems, ICPS, pp.1-6, 27-29 Dec. 2009.

[37] J. Ojeda, M. G. Simoes, G. Li and M. Gabsi, "Design of a Flux-Switching Electrical Generator for Wind Turbine Systems," IEEE Transactions on Industry Applications, 48(6), pp. 1808-1816, Nov. -Dec. 2012.

[38] R. Saou, M. E. Zaïm and K. Alitouche, "Modelling and design of a low speed flux reversal machine," Journal of Electrical Systems, Special Issue No. 1, pp. 18-23, 2009.

[39] Y. Fan, K. T. Chau and M. Cheng, "A new three-phase doubly salient permanent magnet machine for wind power generation," IEEE Transactions on Industry Applications, 42(1), pp. 53-60, Jan. -Feb. 2006.

[40] S. E. Rauch and L. J. Johnson, "Design principles of flux-switching alternators," AIEE Trans., 74(III), pp. 12611268, 1955.

[41] Y. Liao, F. Liang and T. A. Lipo, "A novel permanent magnet motor with doubly salient structure," IEEE Transactions on Industry Applications, 31(5), pp. 1069-1078, Sep/Oct 1995.

[42] I. Boldea, E. Serban, and R. Babau, "Flux reversal stator-PM single-phase generator with controlled DC output," Record of OPTIM-96, Brasov, Romania, pp. 1123-1137, 1996.

[43] W. Hua, P. Su, G. Zhang, G. Zhao and J. G. Webster, Flux-Switching Machines, John Wiley \& Sons, Inc. 1999.

[44] M. Cheng, W. Hua, J. Zhang, and W. Zhao, “Overview of stator-permanent magnet brushless machines," IEEE Transactions on Industrial Electronics, 58(11), pp. 5087-5101, 2011.

[45] J. Zhang, M. Cheng, Z. Chen and W. Hua, "Comparison of stator-mounted permanent-magnet machines based on a general power equation," IEEE Transactions on Energy Conversion, 24(4), pp. 826-834, Dec. 2009.

[46] W. Hua, M. Cheng, Z. Q. Zhu, W. Zhao and X. Kong, "Comparison of electromagnetic performance of brushless motors having magnets in stator and rotor," Journal of Applied Physics, 103, 07F124, Apr. 2008.

[47] Y. Pang, Z. Q. Zhu, D. Howe, S. Iwasaki, R. Deodhar and A. Pride, "Comparative study of flux-switching and interior permanent magnet machines," International Conference on Electrical Machines and Systems, ICEMS, pp.757-762, 8-11 Oct. 2007.

[48] C. Sikder, I. Husain and W. Ouyang, "Cogging torque reduction in flux-switching permanent-magnet machines by rotor pole shaping," IEEE Transactions on Industry Applications, 51(5), pp. 3609-3619, Sept. -Oct. 2015.

[49] D. Wang, X. Wang and S. Y. Jung, "Reduction on cogging torque in flux-switching permanent magnet machine by teeth notching schemes," IEEE Transactions on Magnetics, 48(11), pp. 4228-4231, Nov. 2012.

[50] W. Xu, J. Zhu, Y. Zhang and J. Hu, "Cogging torque reduction for radially laminated flux-switching permanent magnet machine with 12/14 poles," 37th Annual Conference of the IEEE Industrial Electronics Society, IECON, Melbourne, VIC, pp. 3590-3595, 2011.

[51] W. Xu, "Novel decoupling model-based predictive current control strategy for flux-switching permanentmagnet synchronous machines with low torque ripple and switching loss," IEEE Transactions on Applied Superconductivity, 24(5), pp. 1-5, Oct. 2014.

[52] H. Hwang, D. Kim, C. Lee and H. Jin, "Torque ripple reduction in a flux-switching permanent magnet machine targeted at elevator door applications by minimizing space harmonics," IEEE Energy Conversion Congress and Exposition (ECCE), Milwaukee, WI, pp. 1-5, 2016.

[53] W. Hua, Z. Q. Zhu, M. Cheng, Y. Pang and D. Howe, "Comparison of flux-switching and doubly-salient permanent magnet brushless machines,” ICEMS, vol.1, pp.165-170, 27-29 Sept. 2005. 
[54] Y. Amara, E. Hoang, M. Gabsi, M. Lecrivain and S. Allano, "Design and comparison of different flux-switch synchronous machines for an oil breather application," European Transactions on Electrical Power, 15(6), pp.497-511, 2005.

[55] J. D. McFarland, T. M. Jahns and A. M. El-Refaie, "Demagnetization performance characteristics of flux switching permanent magnet machines," International Conference on Electrical Machines (ICEM), Berlin, Germany, pp. 2001-2007, 2014.

[56] D-J Bang, "Design of transverse flux permanent magnet machines for large direct-drive wind turbines", $\mathrm{PhD}$ thesis, Pukyong National University, Korea, 2010.

[57] E. Nipp, "Permanent Magnet motor drives with Switched Stator Windings," Doctoral thesis, Royal Institute of Techonology, Stockholm, May 1999.

[58] E. Hoang, A. h. Ben Ahmed and J. Lucidarme, "Switching flux permanent magnet polyphased synchronous machines," EPE Conference, September 1997.

[59] C. Pollock and M. Wallace, "The flux switching motor, a DC motor without magnets or brushes," Conference Record of the 1999 IEEE Industry Applications Conference, Thirty-Forth IAS Annual Meeting (Cat. No.99CH36370), Phoenix, AZ, vol. 3, pp. 1980-1987, 1999.

[60] C. Pollock and M. Brackley, "Comparison of the acoustic noise of a flux-switching and a switched reluctance drive," IEEE Transactions on Industry Applications, 39(3), pp. 826-834, May-June 2003.

[61] W. Hua, C. Ming, Z. Q. Zhu and D. Howe, "Design of flux-switching permanent magnet machine considering the limitation of inverter and flux-weakening capability," Conference Record of the 2006 IEEE Industry Applications Conference, 41st IAS Annual Meeting., vol.5, pp.2403-2410, 8-12 Oct. 2006.

[62] Z. Q. Zhu, Y. Pang, J. T. Chen, Z. P. Xia and D. Howe, "Influence of design parameters on output torque of flux-switching permanent magnet machines," IEEE Vehicle Power and Propulsion Conference, VPPC, pp.1,6, 3-5 Sept. 2008.

[63] A. S. Thomas, Z. Q. Zhu, R. L. Owen, G. W. Jewell and D. Howe, "Multi-phase flux-switching permanent magnet brushless machine for aerospace application," IEEE Industry Applications Society Annual Meeting, Edmonton, Alta., pp. 1-8, 2008.

[64] J. T. Chen, Z. Q. Zhu, S. Iwasaki and R. Deodhar, "Comparison of losses and efficiency in alternate fluxswitching permanent magnet machines," XIX International Conference on Electrical Machines--ICEM, Rome, pp. 1-6, 2010.

[65] J. T. Chen and Z. Q. Zhu, "Winding configurations and optimal stator and rotor pole combination of fluxswitching PM brushless AC machines," IEEE Transactions on Energy Conversion, 25(2), pp. 293-302, June 2010 .

[66] A. Chen, R. Nilssen and A. Nysveen, "Investigation of a three-phase flux-switching permanent magnet machine for downhole applications," XIX International Conference on Electrical Machines (ICEM), Rome, pp. 1-5, 2010 .

[67] Y. Tang, J. J. H. Paulides, T. E. Motoasca and E. A. Lomonova, "Flux-switching machine with DC excitation," IEEE Transactions on Magnetics, 48(11), pp.3583-3586, Nov. 2012.

[68] L. E. Somesan and I. A. Viorel, "Permanent magnet flux-switching machine, optimal design and performance analysis," Advances in Electrical Engineering, 11(2), pp.46-53, 2013.

[69] A. Fasolo, L. Alberti and N. Bianchi, "Performance comparison between switching-flux and IPM machines with rare-earth and ferrite PMs," IEEE Transactions on Industry Applications, 50(6), pp. 3708-3716, Nov. -Dec. 2014.

[70] Y. J. Zhou and Z. Q. Zhu, "Comparison of wound-field switched-flux machines," IEEE Transactions on Industry Applications, 50(5), pp. 3314-3324, Sept.-Oct. 2014.

[71] T. Raminosoa, A. M. El-Refaie, D. Pan, K-K. Huh, J. P. Alexander, K. Grace, S. Grubic, S. Galioto, P. B. Reddy and X. Shen, "Reduced rare-earth flux-switching machines for traction applications," IEEE Transactions on Industry Applications, 51(4), pp.2959-2971, Aug. 2015.

[72] E. Sulaiman, F. Khan, M. F. Omar, G. M. Romalan and M. Jenal, "Optimal design of wound-field flux switching machines for an all-electric boat," XXII International Conference on Electrical Machines (ICEM), Lausanne, pp. 2464-2470, 2016.

[73] L. Hao, M. Lin, X. Zhao, X. Fu, Z. Q. Zhu and P. Jin, "Static characteristics analysis and experimental study of a novel axial field flux-switching permanent magnet generator," IEEE Transactions on Magnetics, 48(11), pp.4212,4215, Nov. 2012.

[74] M. Lin, L. Hao, X. Li, X. Zhao and Z. Q. Zhu, "A novel axial field flux-switching permanent magnet wind power generator," IEEE Transactions on Magnetics, 47(10), pp.4457-4460, Oct. 2011.

[75] O. Dobzhanskyi, P. Gottipati, E. Karaman, L. Xiaozhong, E. A. Mendrela and A. M. Trzynadlowski, "Multilayer-winding versus switched-flux permanent-magnet ac machines for gearless applications in clean-energy systems," IEEE Transactions on Industry Applications, 48(6), pp.2296,2302, Nov. -Dec. 2012.

[76] J. Yan, H. Lin, Y. Huang, H. Liu, Z. Q. Zhu, "Magnetic field analysis of a novel flux switching transverse flux permanent magnet wind generator with 3-D FEM," International Conference on Power Electronics and Drive Systems (PEDS), pp.332-335, 2-5 Nov. 2009. 
[77] J. H. Kim, Y. Li and B. Sarlioglu, "Novel six-slot four-pole axial flux-switching permanent magnet machine for electric vehicle," IEEE Transactions on Transportation Electrification, 3(1), pp. 108-117, March 2017.

[78] D. Xu, M. Y. Lin, X. H. Fu, L. Hao, W. Zhang and J. L. Zhao, "Design and analysis of a hybrid axial field fluxswitching permanent magnet machine," IEEE International Conference on Applied Superconductivity and Electromagnetic Devices (ASEMD), Shanghai, pp. 314-315, 2015.

[79] C. Yu and S. Niu, "Development of a magnetless flux switching machine for rooftop wind power generation," IEEE Transactions on Energy Conversion, 30(4), pp. 1703-1711, Dec. 2015.

[80] A. S. Thomas, Z. Q. Zhu and G. W. Jewell, "Comparison of flux switching and surface mounted permanent magnet generators for high-speed applications,” Electrical Systems in Transportation (IET), 1(3), pp.111, 116, September 2011.

[81] Y. Wang, J. Sun, Z. Zou, Z. Wang and K. T. Chau, "Design and analysis of a HTS flux-switching machine for wind energy conversion," IEEE Transactions on Applied Superconductivity, 23(3), pp. 5000904-5000904, June 2013.

[82] A. Chen, N. Rotevatn, R. Nilssen and A. Nysveen, "Characteristic investigations of a new three-phase fluxswitching permanent magnet machine by FEM simulations and experimental verification," International Conference on Electrical Machines and Systems, Tokyo, pp. 1-6, 2009.

[83] A. Zulu, B. C. Mecrow and M. Armstrong, "A wound-field three-phase flux-switching synchronous motor with all excitation sources on the stator," IEEE Transactions on Industry Applications, 46(6), pp. 2363-2371, Nov. Dec. 2010.

[84] C. Ditmanson, P. Hein, S. Kolb, J. Mölck and S. Bernet, "A New Modular Flux-Switching Permanent-Magnet Drive for Large Wind Turbines," IEEE Transactions on Industry Applications, 50(6), pp. 3787-3794, Nov. Dec. 2014.

[85] J. H. J. Potgieter, "Optimal topology and critical evaluation of slip synchronous permanent magnet wind generator," PhD Thesis, Electrical and Electronic Engineering, Stellenbosch University, South Africa, April 2014.

[86] R-J Wang and S. Gerber, "Magnetically geared wind generator technologies: opportunities and challenges," Applied Energy, Volume 136, Pages 817-826, 2014.

[87] Y. Duan and D. M. Ionel, "A Review of Recent Developments in Electrical Machine Design Optimization Methods With a Permanent-Magnet Synchronous Motor Benchmark Study," IEEE Transactions on Industry Applications, 49(3), pp. 1268-1275, May-June 2013.

[88] S. Stipetic, W. Miebach and D. Zarko, "Optimization in design of electric machines: methodology and workflow," International Aegean Conference on Electrical Machines \& Power Electronics (ACEMP), Intl Conference on Optimization of Electrical \& Electronic Equipment (OPTIM) \& 2015 Intl Symposium on Advanced Electromechanical Motion Systems (ELECTROMOTION), Side, pp. 441-448, 2015.

[89] P. Zhang, D. M. Ionel and N. A. O. Demerdash, "Saliency ratio and power factor of IPM motors with distributed windings optimally designed for high efficiency and low-cost applications," IEEE Transactions on Industry Applications, 52(6), pp. 4730-4739, Nov. -Dec. 2016.

[90] T. Jahns, "Getting rare-earth magnets out of EV traction machines," IEEE Electrification Magazine, pp. 6-18, March 2017.

[91] S. Gerber, "A finite element based optimisation tool for electrical machines," MSc Thesis, Department of Electrical and Electronic Engineering, Stellenbosch University, Stellenbosch (South Africa) March 2011.

[92] S. Huang, J. Luo, F. Leonardi and T. A. Lipo, "A general approach to sizing and power density equations for comparison of electrical machines," IEEE Transactions on Industry Applications, 34(1), pp. 92-97, Jan/Feb 1998.

[93] VisualDOC User's Manual: Version 8.0, Vanderplaats Research \& Development, Inc., Colorado Springs, CO, February 2017.

[94] J. H. J. Potgieter and M. J. Kamper, "Calculation methods and effects of end-winding inductance and permanent-magnet end flux on performance prediction of nonoverlap winding permanent-magnet machines," IEEE Transactions on Industry Applications, 50(4), 2458-2466, Aug. 2014.

[95] E. B. Sulaiman, T. Kosaka and N. Matsui, "Design study and experimental analysis of wound field flux switching motor for HEV applications," XXth International Conference on Electrical Machines (ICEM), pp.12691275, 2-5 Sept. 2012.

[96] J. Pyrhönen, T. Jokinen and V. Hrabovcova, Design of Rotating Electrical Machines, 5th ed., John Wiley \& Sons, Ltd: UK, 2008.

[97] U. B. Akuru and M. J. Kamper, “Contemporary wind generators," Journal of Energy in Southern Africa, 26(3), pp. 116-124, Aug. 2015.

[98] U. B. Akuru and M. J. Kamper, "Comparative advantage of flux switching PM machines for medium-speed wind drives," International Conference on the Domestic Use of Energy (DUE), pp.149,154, March 31-April 1 2015.

[99] G. N. Vanderplaats, Multidiscipline Design Optimisation, First Edition. Vanderplaats Research \& Development, Inc., 2007. 
[100] E. Ilhan, M. F. J. Kremers, E. T. Motoasca, J. J. H. Paulides and E. A. Lomonova, "Sensitivity analysis for phase inductances in Flux-Switching PM machines," XXth International Conference on Electrical Machines, Marseille, pp. 763-768, 2012.

[101] U.B. Akuru and M.J. Kamper, "Evaluation of flux switching PM machines for medium-speed wind generator drives," 2015 IEEE Energy Conversion Congress and Exposition (ECCE), pp.1925-1931, 20-24 Sept. 2015.

[102] Z. Q. Zhu, Z. Z. Wu, D. J. Evans, and W. Q. Chu, “A wound field switched flux machine with field and armature windings separately wound in double stators," IEEE Transactions on Energy Conversion, 30(2), pp. 772-783, Jun. 2015.

[103] L. Shao, W. Hua, Z. Q. Zhu, X. Zhu, M. Cheng and Z. Wu, “A novel flux-switching permanent magnet machine with overlapping windings," IEEE Transactions on Energy Conversion, 32(1), pp. 172-183, March 2017.

[104] Z. Q. Zhu, M. M. J. Al-Ani, X. Liu and B. Lee, "A mechanical flux weakening method for switched flux permanent magnet machines," IEEE Transactions on Energy Conversion, 30(2), pp. 806-815, June 2015.

[105] R. Cao, C. Mi and M. Cheng, "Quantitative comparison of flux-switching permanent-magnet motors with interior permanent magnet motor for EV, HEV, and PHEV applications," IEEE Transactions on Magnetics, 48(8), pp. 2374-2384, August 2012.

[106] C. Anyuan, N. Rotevatn, R. Nilssen, A. Nysveen, "Characteristic investigations of a new three-phase fluxswitching permanent magnet machine by FEM simulations and experimental verification," International Conference on Electrical Machines and Systems, ICEMS, pp. 1-6, 15-18 Nov., 2009.

[107] K. Deb, A. Pratap, S. Agarwal and T. Meyarivan, "A fast and elitist multiobjective genetic algorithm: NSGAII,” IEEE Transactions on Evolutionary Computation, 6(2), pp. 182-197, Apr. 2002.

[108] J. Le Besnerais, V. Lanfranchi,M. Hecquet, and P. Brochet, "Multiobjective optimization of induction machines including mixed variables and noise minimization," IEEE Transactions on Magnetics, 44(6), pp. 1102-1105, Jun. 2008.

[109] X. Jannot, J. Vannier, C. Marchand, M. Gabsi, J. Saint-Michel, and D. Sadarnac, "Multiphysic modeling of a high-speed interior permanent magnet synchronous machine for a multiobjective optimal design," IEEE Transactions on Energy Conversion, 26(2), pp. 457-467, Jun. 2011.

[110] X. Wang, T. D. Strous, D. Lahaye, H. Polinder and J. A. Ferreira, "Modeling and Optimization of Brushless Doubly-Fed Induction Machines Using Computationally Efficient Finite-Element Analysis," IEEE Transactions on Industry Applications, 52(6), pp. 4525-4534, Nov. -Dec. 2016.

[111] U. B. Akuru and M. J. Kamper, "Performance comparison of optimum wound-field and ferrite PM flux switching machines for wind energy applications," XXII International Conference on Electrical Machines (ICEM), Lausanne, pp. 2478-2485, 2016.

[112] U. B. Akuru and M. J. Kamper, "Investigation of low-cost PM flux switching machine for medium-speed geared wind energy applications," South African Universities Power Engineering Conference (SAUPEC), pp. 613-618, Stellenbosch, South Africa, 30 Jan. -1 Feb. 2017.

[113] J. H. J. Potgieter and M. J. Kamper, "Design of New Concept Direct Grid-Connected Slip-Synchronous Permanent-Magnet Wind Generator," IEEE Transactions on Industry Applications, 48(3), pp.913-922, May-June 2012.

[114] R-J. Wang, L. Bronn, S. Gerber and P.M. Tlali, "Design and evaluation of a disc-type magnetically geared PM wind generator," Fourth International Conference on Power Engineering, Energy and Electrical Drives (POWERENG), pp.1259-1264, 13-17 May 2013.

[115] I. Boldea, L. Tutelea and F. Blaabjerg, "High power wind generator designs with less or no PMs: An overview," 17th International Conference on Electrical Machines and Systems (ICEMS), pp.1-14, 22-25 Oct. 2014

[116] A. Dupas, E. Hoang, S. Hlioui, B. Gaussens, M. Lécrivain and M. Gabsi, "Performances of a hybrid excited flux-switching DC-alternator: Analysis and experiments," International Conference on Electrical Machines (ICEM), Berlin, pp. 2644-2649, 2014.

[117] E. Hoang, B. Gaussens, M. Lecrivain and M. Gabsi, "Proposal to increase the convertible power by an hybrid excitation flux switching synchronous machine associated with an voltage inverter--application in hybrid or electric vehicle," Tenth International Conference on Ecological Vehicles and Renewable Energies (EVER), Monte Carlo, pp. 1-7, 2015.

[118] G-J Li, Z. Zhu and G. Jewell, "Performance investigation of hybrid excited switched flux permanent magnet machines using frozen permeability method," IET Electric Power Applications, 9(9), pp.586-594, 112015.

[119] Y. Tang, E. Ilhan, J.J.H. Paulides and E.A. Lomonova, "Design considerations of flux-switching machines with permanent magnet or DC excitation," 15th European Conference on Power Electronics and Applications (EPE), pp.1-10, 2-6 Sept. 2013.

[120] P. Andrada and F. Martinez, "Flux switching alternators for small wind generation," ICREPQ'16, Madrid, Spain, 2016.

[121] A. M. El-Refaie, "Growing role of electrical machines and drives in electrification," XXII International Conference on Electrical Machines (ICEM), Lausanne, pp. 364-370, 2016. 
[122] L. Shao, W. Hua, N. Dai, M. Tong and M. Cheng, "Mathematical modeling of a 12-phase flux-switching permanent-magnet machine for wind power generation," IEEE Transactions on Industrial Eleictronics, 63(1), pp. 504-516, Jan. 2016.

[123] T. Kosaka, N. Matsui, Y. Kamada and H. Kajiura, "Experimental drive performance evaluation of high power density wound field flux switching motor for automotive applications," 7th IET International Conference on Power Electronics, Machines and Drives (PEMD), Manchester, United Kingdom, pp.1-6, 8-10, April 2014.

[124] Y. Y. Xia, J. E. Fletcher, S. J. Finney, K. H. Ahmed and B. W. Williams, "Torque ripple analysis and reduction for wind energy conversion systems using uncontrolled rectifier and boost converter," IET Renewable Power Generation, 5(5), pp. 377-386, September 2011.

[125] U. B. Akuru and M. J. Kamper, "Formulation and multi-objective design optimisation of wound-field flux switching machines for wind energy drives," IEEE Transactions on Industrial Electronics, In Press, 2017.

[126] P. Zhou, D. Lin, Y. Xiao, N. Lambert and M. A. Rahman, "Temperature-dependent demagnetization model of permanent magnets for finite element analysis," IEEE Transactions on Magnetics, 48(2), pp. 1031-1034, Feb. 2012.

[127] O. Kudrjavtsev, A. Kilk, T. Vaimann, A. Belahcen and A. Kallaste, "Implementation of different magnetic materials in outer rotor PM generator," IEEE 5th International Conference on Power Engineering, Energy and Electrical Drives (POWERENG), Riga, pp. 74-78, 2015.

[128] G-H. Kang, J. Hur, H. Nam, J-P. Hong and G-T. Kim, "Analysis of irreversible magnet demagnetization in line-start motors based on the finite-element method," IEEE Transactions on Magnetics, 39(3), pp. 1488-1491, May 2003.

[129] A. El-Wakeel, "Design optimisation of electric machines," $4^{\text {th }}$ International Conference on Electrical Engineering (ICEENG), Military Technical College, Cairo, Egypt, 23-25Nov. 2004.

[130] SEMFEM documentation. [Online]. Available: www0.sun.ac.za/semfem/index.html

[131] J. Salvatore, G. Turner, H. Boyle, E. Nekhaev, A. Clerici, and S. Ulreich (Eds.), World energy: perspective cost of energy technologies, World Energy Council, 2013. [Online]. Available: http://www.worldenergy.org

[132] J. Kolehmainen and J. Ikaheimo, "Motors with buried magnets for medium-speed applications," IEEE Transactions on Energy Conversion, 23(1), pp.86-91, March 2008.

[133] Z. Q. Zhu, J. X. Shen and D. Howe, "Flux-weakening characteristics of non-sinusoidal back-EMF PM machines in brushless DC and AC modes," Journal of Asian Electric Vehicles, pp. 919-925, 4(2), December 2006.

[134] B. Štumberger, G. Štumberger, M. Hadžiselimović and M. Trlep, "Performance comparison of three-phase flux reversal permanent magnet motors with surface and inset permanent magnets," PRZEGLAD ELEKTROTECHNICZN, pp. 107-110, R. 82 NR 12/2006.

[135] K. Kim, L. Kab-Jae and J. Lee. "Dynamic analysis of spoke type permanent magnet generator with large overhang," Digests of the IEEE International Magnetics Conference, INTERMAG, Asia, pp.671-672, 4-8 April 2005.

[136] J. Marques, H. Pinheiro, H. Grundling, J. Pinheiro and H. Hey, "A survey on variable-speed wind turbine system," Proceedings of Brazilian conference of electronics of power, vol. 1. p. 732-8, 2003.

[137] S. Muller, M. Deicke, R. W. De Doncker, "Doubly fed induction generator systems for wind turbines," IEEE Industry Applications Magazine, 8(3), pp. 26-33, May 2002.

[138] M. Mabhula and M. J. Kamper, "Saliency and mutual inductance effect in cylindrical wound-rotor synchronous motor," IEEE Workshop on Electrical Machines Design, Control and Diagnosis (WEMDCD), pp. 152-157, Nottingham, 2017.

[139] W. Hua and M. Cheng, "Cogging torque reduction of flux-switching permanent magnet machines without skewing," 2008 International Conference on Electrical Machines and Systems, Wuhan, 2008, pp. 3020-3025.

[140] D. Wang, X. Wang, S-Y. Jung, "Reduction on Cogging Torque in Flux-Switching Permanent Magnet Machine by Teeth Notching Schemes," IEEE Transactions on Magnetics, 48(11), pp. 4228-4231, Nov. 2012.

[141] J. X. Shen and W. Z. Fei, "Permanent magnet flux switching machines - Topologies, analysis and optimization," 4th International Conference on Power Engineering, Energy and Electrical Drives, Istanbul, 2013, pp. 352-366.

[142] C. Sikder, I. Husain and W. Ouyang, "Design Control and Implementation of a Non-Rare-Earth Flux Switching Permanent Magnet Machine," IEEE Energy Conversion Congress and Exposition (ECCE), Milwaukee, WI, USA, 2016.

[143] M. G. Simões, F. A. Farret and F. Blaabjerg, "Small Wind Energy Systems," Electric Power Components and Systems, 43(12), Taylor \& Francis, 1388-1405, 2015.

[144] W. Fei, P. C. K. Luk and J. Shen, "Torque analysis of permanent magnet flux switching machines with rotor step skewing," IEEE Transactions on Magnetics, 48(10), 2664-2673, 2012.

[145] X. Zhu, W. Hua, Z. Wu, W. Huang, H. Zhang and M. Cheng, “Analytical Approach for Cogging Torque Reduction in Flux-Switching Permanent Magnet Machines Based on Magneto Motive Force-Permeance Model," IEEE Transactions on Industrial Electronics, in press, 2017.

[146] S. D. Umans, Fitzgerald \& Kingsley's Electric Machinery, 7th ed., McGraw-Hill International Edition, New York, 2014 
[147] A. Zulu, B. C. Mecrow and M. Armstrong, "Prediction of performance of a wound-field segmented-rotor fluxswitching synchronous motor using a dq-equivalent model," The XIX International Conference on Electrical Machines (ICEM 2010), Rome, pp. 1-6, 2010.

[148] T. L. Balyovski, E. Ilhan, Y. Tang, J. J. H. Paulides, C. G. E. Wijnands and E. A. Lomonova, "Control of DCexcited flux switching machines for traction applications," 2014 Ninth International Conference on Ecological Vehicles and Renewable Energies (EVER), Monte-Carlo, pp. 1-5, 2014. 


\section{APPENDICES}

\section{A1.1 Operation Principle of Flux Switching Machines}

The basic operation of FSMs can be fully described by the single-phase flux-switch alternator originally proposed by Rauch and Johnson (1955) [40]. The operating concept revolves around the change in permeance in the stationary core due to relative movement of the rotating member (rotor). As a result of this, if the armature coils in the stationary core (stator) is appropriately placed, such that it respects Faraday's law of electromagnetic induction, it produces a change in flux linkage produced by the field source $\left(\psi_{M}\right)$ with respect to the rotor position $(\theta)$ at a given electrical speed $\left(\omega_{e}\right)$ in $\mathrm{rad} / \mathrm{s}$; thus, yielding an induced voltage, $e_{0}$, in the coil given as

$$
e_{0}=-\omega_{e} \frac{d \psi_{M}}{d \theta} .
$$

As an example, Rauch and Johnson's (1955) [40] single-phase flux-switch alternator is redesigned and simulated in two-dimensional (2-D) finite-element analyses (FEA). As seen in Fig. A1.1, to achieve flux switching, adjacent PMs are arranged such that their magnetic fields are in opposition. It is also observed that each PM is surrounded by adjacent stator teeth that are owned by the same coil.

Hence, when driven by a prime-mover, the two rotor poles align with the stator teeth in position "A" as depicted in Fig. A1.1(a), which shows the flux from the north-poles of "PMs 1 and 2" going to the rotor tooth in alignment to one of its members at that point, through an adjacent stator pole. At the same time, Fig. A1.1(a) also shows that the south-poles of "PM 1 and 2" receive flux from the rotor tooth in alignment to one of its member, through an adjacent stator pole.

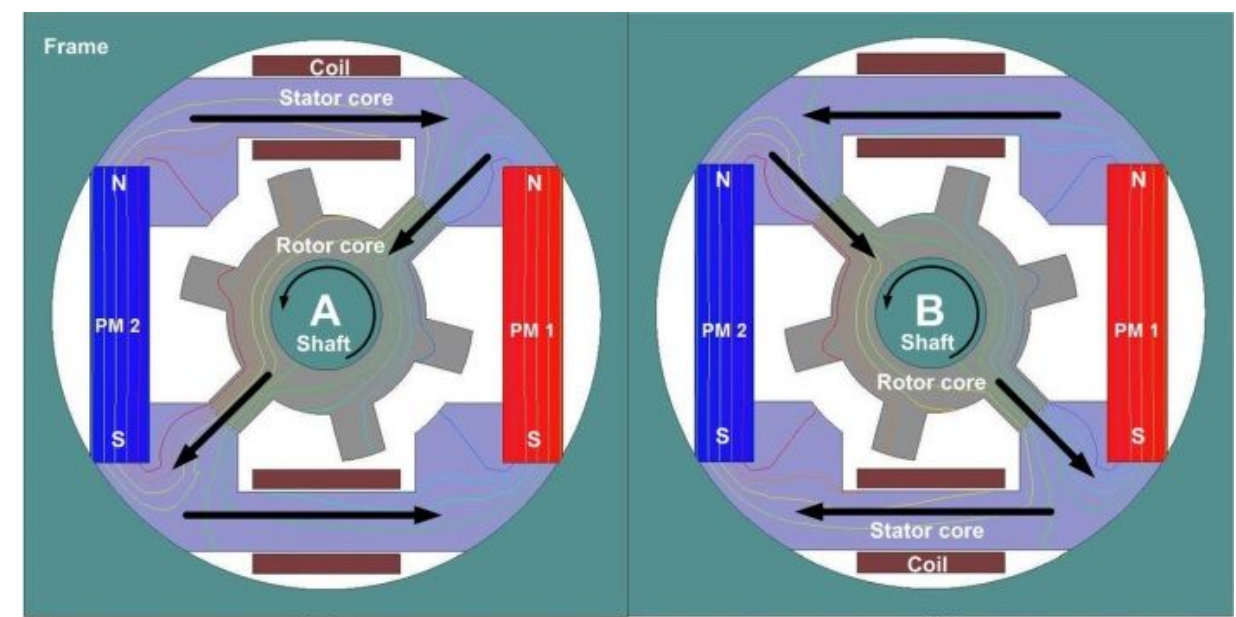

(a) (b)

Fig. A1.1. An illustration of the flux-switch concept in 2-D FEA (with field distributions) considered at different rotor positions such as: (a) A, and (b) B. 


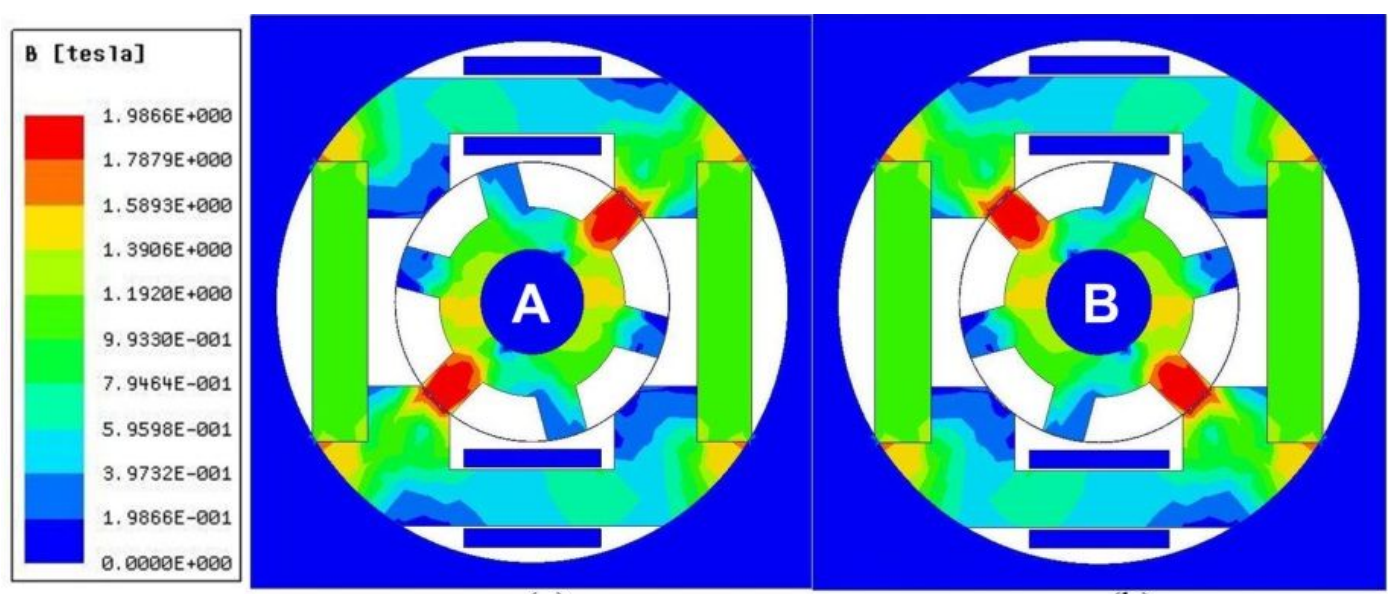

(a)

(b)

Fig. A1.2. Flux density map of 4-stator slots/6-rotor poles single-phase FSM at different rotor positions such as: (a) position "A", and (b) position "B".

Subsequently, the process is repeated vice versa when the rotor position shifts to "B" as illustrated in Fig. A1.1(b); clearly displaying the "flux switching" concept. Merely looking at the flux directions elucidated by the course of the arrowheads, the flux switching concept is confirmed.

The flux density maps of both evaluated positions are further represented in Fig. A1.2. It is observed that the movement of the rotor from point " $\mathrm{A}$ " to " $\mathrm{B}$ " results in a periodic change of the flux linkage which induces a total voltage, $e_{0}$, in the two coils as referred in (A1.1). Note that, the coils as shown in Fig. A1.1 are connected in series. Thus, by connecting a load, $R$ across the terminals, (A1.1) is modified to

$$
e_{s}=-\omega_{e} \frac{d \psi_{T}}{d \theta}-i_{s} R
$$

whereby $e_{s}$ is now referred to as the terminal voltage, $\psi_{T}$ is the effective flux linkage, and $i_{s}$ is the current flowing through the load.

The waveforms shown in Fig. A1.3 are the resulting electromagnetic quantities measured across the machine's terminals when operating under rated load. Note that, the expressed flux linkage, induced voltage and load current are essentially sinusoidal.

Likewise, to achieve three-phase operation of the single-phase basic demonstration, the coils are simply duplicated for the right number of poles-slots combination, and arranged in three similar groups, each differentiated $120^{\circ}$ apart, spatially. Thus by applying a set of three-phase current, each equal in magnitude and differing in phase by $120^{\circ}$, the three-phase topology is evinced. Note that, by three-phase, it means that the three-phase set of the electromagnetic quantities shown in Fig. A1. 3 are produced within a set of equal coils (if more than one is used per phase).

Therefore, in agreement with Chen and Zhu (2010) [65] and Ditmanson et al (2013) [84], increasing the FSM phase number from single-phase to a multi-phase topology, is just a matter of the 
stator and rotor pole number combinations, e.g., a 12/10 topology can realise a three-phase set of balanced symmetrical quantities with a non-zero rated average electromagnetic torque. Hence, such three-phase operational conceptualisations, should the reader be interested, can be mined from the following studies: Hua et al (2005) [53], Hoang, Ben Ahmed and Lucidarme (1997) [58], Hua et al (2006) [61] and Chen et al (2009) [82], to mention a few.

\section{A1.2 Sizing Procedure of Flux Switching Machines}

As already known, the sizing method has been applied to PM-FSMs in Zhang et al (2009) [45], Hua et al (2006) [61] and Somesan and Viorel (2013) [68]. When available to the designer, the layouts for the three-phase 12/10 and 12/14 PM-FSM is realisable with only few pre-defined design specifications such as output power, rated speed, efficiency, electrical loading, etc.

The sizing to power expression proposed in Hua et al (2006) [61] for PM-FSMs is given as

$$
D_{\text {out }}=\sqrt[3]{\frac{4 \tau_{m} N_{s}}{\sqrt{2} \pi^{2} N_{r} \kappa_{e} \kappa_{L} \Lambda_{0}^{3} A_{s} \dot{B} \eta \eta c_{s}}}
$$

where $D_{\text {out }}$ is the stator outer diameter, $N_{s}$ is the number of slots for the phase windings, $\kappa_{e}$ is a factor to account for some leakage, $A_{s}$ is the electrical loading of the phase windings, $\dot{B}_{9}$ is the peak airgap flux density, $\eta$ is the efficiency of the machine and $c_{s}$ is the stator tooth arc factor, generally prescribed at 0.25 for the FSM topologies considered in this thesis.
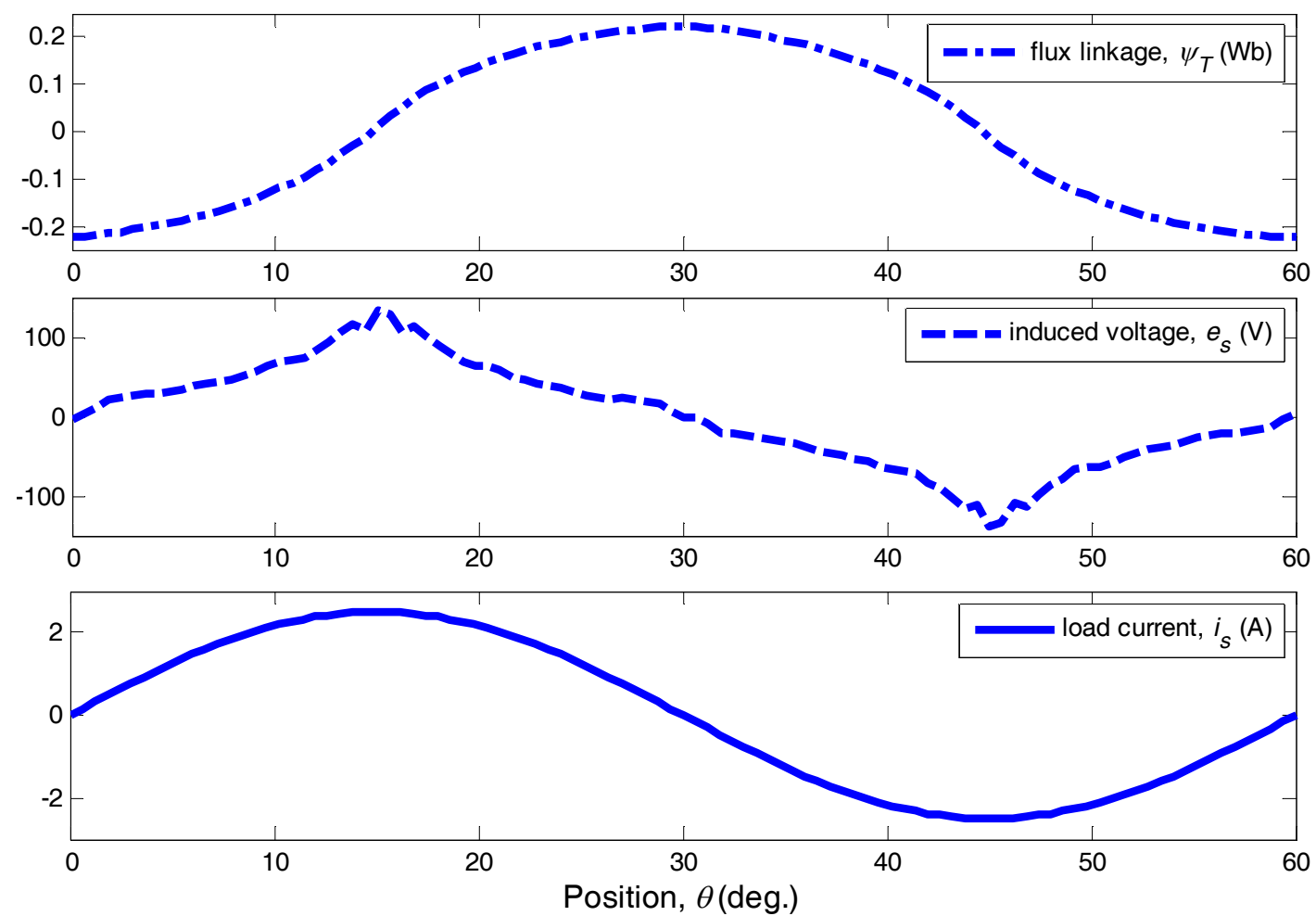

Fig. A1.3. Sinusoidal expression of flux linkage, induced voltage, and load current plotted against the rotor position of single-phase flux-switch alternator, at $400 \mathrm{r} / \mathrm{min}$. 
The mechanical torque $\tau_{m}$, split ratio $\Lambda_{0}$ and aspect ratio $\kappa_{L}$ are derived as

$$
\tau_{m}=\frac{\mathrm{P}_{\text {out }}}{\omega_{\mathrm{s}}} ; \quad \Lambda_{0}=\frac{D_{\text {in }}}{D_{\text {out }}} ; \quad \kappa_{L}=\frac{l_{\text {st }}}{D_{\text {in }}},
$$

where $\mathrm{P}_{\text {out }}$ is the prescribed generator outer power, $\omega_{s}$ is the mechanical speed in rad $/ \mathrm{s}, l_{s t}$ is the stack length of the laminations, and $D_{\text {in }}$ is the stator interior diameter.

Some of the parameters like $\kappa_{e}, \kappa_{L}, \Lambda_{0}, \dot{B}_{9}$ and $A_{s}$ are easy to set initially as they are well referenced in the literature. For example, $A_{S}$ is prescribed at $35-65 \mathrm{kA} / \mathrm{m}$ as detailed in Pyrhönen, Jokinen and Hrabovcova (2008) [96], for salient-pole synchronous machines like FSMs.

In respect of the WF-FSM topologies, the modification required in (A1.4) is to redefine $A_{s}$ as

$$
A_{\Sigma}=A_{S}+A_{F},
$$

whereupon $A_{F}$ is an additional electrical loading defined by the presence of the field coils. Thus, $A_{\Sigma}$ is substituted for $A_{s}$ in (A1.4) to make it applicable to WF-FSMs.

After determining $D_{\text {out }}$ using (A1.4), the remaining geometrical parameters of the FSM such as the stator slot opening width $\left(b_{s l s}\right)$, stator pole width $\left(b_{p s}\right)$, stator yoke height $\left(h_{y s}\right)$, rotor pole width $\left(b_{p r}\right)$, PM length $\left(b_{p m}\right)$ in PM-FSMs or field core iron length $\left(b_{F}\right)$ in WF-FSMs, and rotor yoke height $\left(h_{y r}\right)$ are intialised as follows:

$$
b_{p s}=b_{p m}=b_{s l s}=h_{y s}=b_{p r}=h_{y r}=\frac{\tau_{s}}{4},
$$

whereupon $\tau_{s}$ is the stator pole pitch defined as

$$
\tau_{s}=\frac{\pi D_{\text {in }}}{N_{S}}
$$

Based on these approximations, the stator and rotor topology, for example, of a 12/10 PMFSM is conceived as shown in Figs. A1.4(a) and A1.5. Besides, with this initial dimensioning, the PM-FSM model is able to satisfy the prescribed design requirements, to a great extent. On the other hand, the stator segment of the WF-FSM design proof is as shown in Fig. A1.4(b).

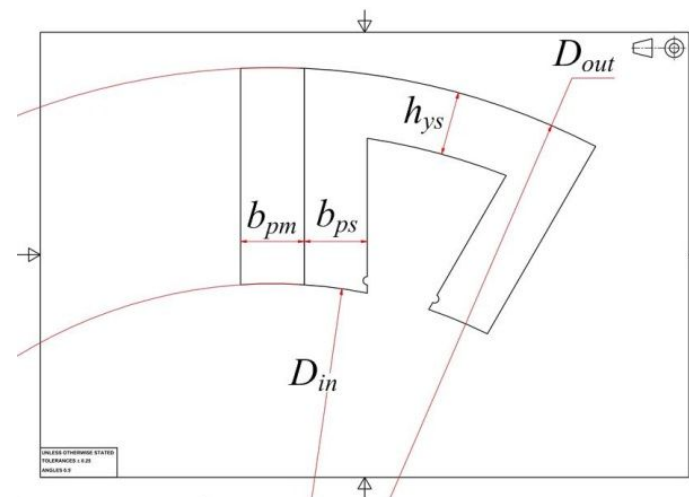

(a)

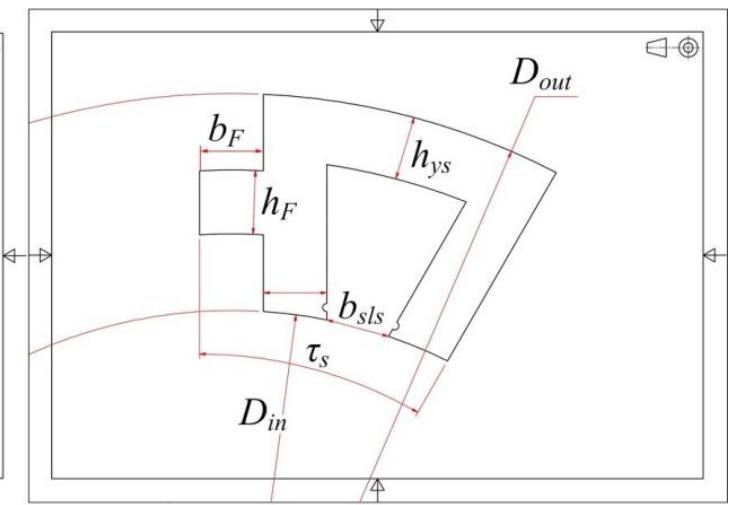

(b)

Fig. A1.4. Conceived geometry of the stator segments for: (a) PM-FSM design, and (b) WF-FSM design. 


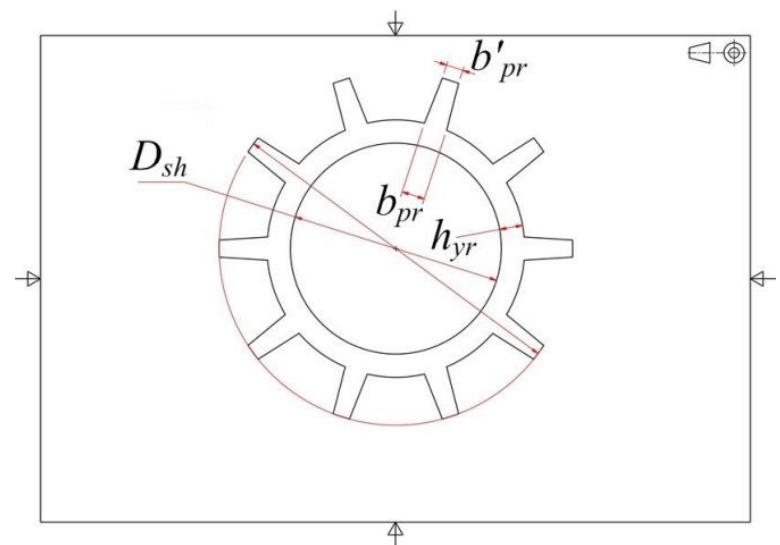

Fig. A1.5. Conceived geometry of the simple rotor lamination. 